\title{
University of Illinois at Chicago
}

\section{PLANTS OF IRIAN JAYA}

\section{APOCYNACEAE}

Det. By:

Field Char: Uncommon understory tree $10 \mathrm{~m}$ tall, bole straight, $10 \mathrm{~cm}$ dbh; bark gray, smooth; calyx whitish, corolla tube pale green, segments white, twisted; latex white.

Locality: Irian Jaya: Sarmi Province, slopes of small mountain ridge rising from coastal plain, "Gunung Top", west of Sungai Tor, east of Sungai Waske, vicinity of Sewan village near Titfe stream.

Habitat: Primary lowland forest, canopy 10-60 m tall. Associates diverse, include

Sterculia, Knema, Polyalthia,

7.izyphus, Pometia, etc.

$10-100 \mathrm{~m}, 2^{\circ} 4^{\prime} \mathrm{S} X 138^{\circ} 46^{\prime} \mathrm{E}$

McDonald \& Ismail 3690, May 23, 1993

This is a voucher specimen for anticancer and anti-AIDS screening NCI Sample Number(s): U44Z4945-L, U44Z4946-M U44Z4947-N.

Collected under the sponsorship of: U.S. National Cancer Institute, University of Illinois at Chicago, Amold Arboretum (Harvard University), Field Museum of Natural History and BO/LIPI. 


\section{University of Illinois at Chicago}

\section{PLANTS OF IRIAN JAYA}

\section{APOCYNACEAE}

Lepinia solomonensis Hmsl.

Det. By: A. Me Donald

Field Char: Uncommon understory tree $12 \mathrm{~m}$ tall, branching midway up, lateral stems

perpendicular to bole, bole straight, $6 \mathrm{~cm} \mathrm{dbh}$;

bark gray, rough; corolla white, segments twisted.

Locality: Irian Jaya: Sarmi Province, slopes of small mountain ridge rising from coastal plain, "Gunung Top", west of Sungai Tor, east of Sungai Waske, vicinity of Sewan village near Titfe stream.

Habitat: Primary lowland forest, canopy $10-60 \mathrm{~m}$ tall. Associates diverse, include

Sterculia, Knema, Polyalthia,

Zizyphus, Pometia, etc.

$10-100 \mathrm{~m}, 2^{\circ} 4^{\prime} \mathrm{S} X 138^{\circ} 46^{\prime} \mathrm{E}$

McDonald \& Ismail 3691, May 23, 1993

This is a voucher specimen for anticancer and anti-AIDS screening NCI Sample Number(s): U44Z4948-O, U44Z4949-P

U44Z4950-Q.

Collected under the sponsorship of: U.S. National Cancer Institute, University of Illinois at Chicago, Arnold Arboretum (Harvard University), Field Museum of Natural History and BO/LIPI. 


\section{University of Illinois at Chicago}

\section{PLANTS OF IRIAN JAYA}

\section{ARALIACEAE}

?Fatsia

Det. By:

Field Char: Occasional riparian tree, branching from base thrice, boles cylindrical, to $20 \mathrm{~cm}$ diam.; bark smooth, pale gray; fruits maturing from pale green to dark purple.

Locality: Irian Jaya: Sarmi Province, slopes of small mountain ridge rising from coastal plain, "Gunung Top", west of Sungai Tor, east of Sungai Waske, vicinity of Sewan village near Titfe stream.

Habitat: Primary lowland forest, canopy 10-60 m tall. Associates diverse, include

Sterculia, Knema, Polyalthia,

Zizyphus, Pometia, etc.

$10-100 \mathrm{~m}, 2^{\circ} 4^{\prime} \mathrm{S} X 138^{\circ} 46^{\prime} \mathrm{E}$

McDonald \& Ismail 3692, May 23, 1993

This is a voucher specimen for anticancer and anti-AIDS screening NCI Sample Number(s): U44Z4951-R, U44Z4952-S U44Z4953-T.

Collected under the sponsorship of: U.S. National Cancer Institute, University of Illinois at Chicago, Arnold Arboretum (Harvard University), Field Museum of Natural History and BO/LIPI. 


\section{PLANTS OF INDONESIA}

Rubiaceae

Cat. \# 3693

Nauclea perpicuinervia Merr. \&P.

Det. By: McDonald 1993

Locality:

New Guinea; Sarmi,

Coastal plain and Gunung Top, W of Sungai Tor, E

of Sungai Waske, vic. of Sewan village on Titve

Stream.

$2^{\circ} 14^{\prime} \mathrm{S} 138^{\circ} 46^{\prime}$ E Alt: $10-100 \mathrm{~m}$

Habitat:

Primary lowland forest, canopy 10-60 m high; associates diverse, including Sterculia, Knema, Polyalthia, Pometia, Ziziphus.

Field Char:

Riparian tree $12 \mathrm{~m}$ high, branching midway up; bole straight, cylindrical, mottled tan and gray, bark smooth; flowers cream, style white, stipules pale green, yellowish at base, coriaceous, ridged.

Notes:

NCI voucher

McDonald \& Ismail 3693

24 May 1993

\section{HERBARIUM BOGORIENSE HARVARD UNIVERSITY HERBARIA}

\section{PLANTS OF INDONESIA}

Verbenaceae

Cat. \# 3694

\section{Callicarpa pentandra Roxb.}

Det. By: McDonald 1993

Locality:

New Guinea; Sarmi,

Coastal plain and Gunung Top, W of Sungai Tor, E of Sungai Waske, vic. of Sewan village on Titve

Stream.

$2^{\circ} 14^{\prime} \mathrm{S} 138^{\circ} 46^{\prime} \mathrm{E}$ Alt: $10-100 \mathrm{~m}$

Habitat:

Primary lowland forest, canopy 10-60 m high; associates diverse, including Sterculia, Knema, Polyalthia, Pometia, Ziziphus.

Field Char:

Occasional tree $10 \mathrm{~m}$ high, branching above, foliage light green; bole straight, cylindrical; bark pale gray, smooth; calyx green during anthesis, corolla tube white, limb segments bright lavender, anthers dark lavender; fruits rotund, red-pink.

Notes:

NCI voucher

McDonald \& Ismail 3694 


\section{PLANTS OF INDONESIA}

Euphorbiaceae

Cat. \# 3695

Endospermum formicarum Becc.

Det. By: McDonald 1993

Locality:

New Guinea; Sarmi,

Coastal plain and Gunung Top, W of Sungai Tor, E of Sungai Waske, vic. of Sewan village on Titve

Stream.

$2^{\circ} 14^{\prime} \mathrm{S} 138^{\circ} 46^{\prime} \mathrm{E}$ Alt: $10-100 \mathrm{~m}$

Habitat:

Primary lowland forest, canopy 10-60 m high; associates diverse, including Sterculia, Knema, Polyalthia, Pometia, Ziziphus.

Field Char:

Occasional riparian tree, $9 \mathrm{~m}$ tall, branching at base, boles to $15 \mathrm{~cm}$ diam; stems hollow and perforate, filled with aggressive ants; calyx and fruits green, residual stigma yellow.

McDonald \& Ismail $3695 \quad 24$ May 1993

\section{HERBARIUM BOGORIENSE HARVARD UNIVERSITY HERBARIA}

\section{PLANTS OF INDONESIA}

Cat. \# 3696

Villebrunea sp.

Det. By: McDonald 1993

Locality:

New Guinea; Sarmi,

Coastal plain and Gunung Top, W of Sungai Tor, E of Sungai Waske, vic. of Sewan village on Titve

Stream.

$2^{\circ} 14^{\prime}$ 'S $138^{\circ} 46^{\prime}$ E Alt: $10-100 \mathrm{~m}$

Habitat:

Primary lowland forest, canopy 10-60 m high; associates diverse, including Sterculia, Knema, Polyalthia, Pometia, Ziziphus.

Field Char:

Common riparian tree, $5 \mathrm{~m}$ tall, branching profusely from base, basal stems to $10 \mathrm{~cm}$ diam; bark pale gray with shallow, longitudinal lenticels; inflorescence clavate, head dark maroon. 


\section{PLANTS OF INDONESIA}

Euphorbiaceae

Cat. \# 3697

Macaranga densiflora Warb.

Det. By: McDonald 1993

Locality:

New Guinea; Sarmi,

Coastal plain and Gunung Top, W of Sungai Tor, E

of Sungai Waske, vic. of Sewan village on Titve

Stream.

$2^{\circ} 14^{\prime} \mathrm{S} 138^{\circ} 46^{\prime} \mathrm{E}$ Alt: $10-100 \mathrm{~m}$

Habitat:

Primary lowland forest, canopy $10-60 \mathrm{~m}$ high; associates diverse, including Sterculia, Knema, Polyalthia, Pometia, Ziziphus.

Field Char:

Uncommon riparian tree, $11 \mathrm{~m}$ tall, branching

midway up, bole contoured, covered with papery ant nests; bark pale, gray, smooth; inflorescence

yellowish, peduncles and petioles reddish.

McDonald \& Ismail 3697

24 May 1993

HERBARIUM BOGORIENSE

HARVARD UNIVERSITY HERBARIA

\section{PLANTS OF INDONESIA}

Myristicaceae

Cat. \# 3698

Myristica sp.

Det. By: McDonald 1993

Locality:

New Guinea; Sarmi,

Coastal plain and Gunung Top, W of Sungai Tor, E

of Sungai Waske, vic. of Sewan village on Titve

Stream.

$2^{\circ} 14^{\prime} \mathrm{S} 138^{\circ} 46^{\prime} \mathrm{E}$ Alt: $10-100 \mathrm{~m}$

Habitat:

Primary lowland forest, canopy $10-60 \mathrm{~m}$ high; associates diverse, including Sterculia, Knema, Polyalthia, Pometia, Ziziphus.

Field Char:

Common, short riparian tree, $8 \mathrm{~m}$ tall, bole straight, cylindrical, $10 \mathrm{~cm}$ diam, inner bark red, outer

brownish, moderately rough; flowers on short shoots, cream.

Notes:

$\mathrm{NCI}$ voucher

McDonald \& Ismail 3698

24 May 1993

HERBARIUM BOGORIENSE HARVARD UNIVERSITY HERBARIA 


\section{PLANTS OF INDONESIA}

Lecythidaceae

Cat. \# 3699

\section{Planchonia papuana Knuth}

Det. By: McDonald 1993

Locality:

New Guinea; Sarmi,

Coastal plain and Gunung Top, W of Sungai Tor, E of Sungai Waske, vic. of Sewan village on Titve

Stream.

$2^{\circ} 14^{\prime} \mathrm{S} 138^{\circ} 46^{\prime}$ E Alt: $10-100 \mathrm{~m}$

Habitat:

Primary lowland forest, canopy 10-60 m high; associates diverse, including Sterculia, Knema, Polyalthia, Pometia, Ziziphus.

Field Char:

Riparian canopy tree $22 \mathrm{~m}$ tall, branching midway up; bole straight, cylindrical, bark red-brown with narrow longitudinal grooves, flaky; fruits immature, green, style persistent.

Notes:

$\mathrm{NCI}$ voucher

McDonald \& Ismail 3699

24 May 1993

\section{HERBARIUM BOGORIENSE HARVARD UNIVERSITY HERBARIA}

\section{PLANTS OF INDONESIA}

Meliaceae

Cat. \# 3700

Vavaea amicorum Benth.

Det. By: McDonald 1993

Locality:

New Guinea; Sarmi,

Coastal plain and Gunung Top, W of Sungai Tor, E

of Sungai Waske, vic. of Sewan village on Titve

Stream.

$2^{\circ} 14^{\prime} \mathrm{S} 138^{\circ} 46^{\prime} \mathrm{E}$ Alt: $10-100 \mathrm{~m}$

Habitat:

Primary lowland forest, canopy $10-60 \mathrm{~m}$ high; associates diverse, including Sterculia, Knema, Polyalthia, Pometia, Ziziphus.

Field Char:

Uncommon, riparian canopy tree, $25 \mathrm{~m}$ tall, branching from base to summit; bole straight, cylindrical, bark pale gray, smooth; fruits maturing from orange to red to purple.

Notes:

$\mathrm{NCI}$ voucher

McDonald \& Ismail 3700

24 May 1993

HERBARIUM BOGORIENSE HARVARD UNIVERSITY HERBARIA 


\section{PLANTS OF INDONESIA}

Cat. \# 3701

\section{Clerodendron confusum Hall. $f$.}

Det. By: McDonald 1993

Locality:

New Guinea; Sarmi,

Coastal plain and Gunung Top, W of Sungai Tor, E of Sungai Waske, vic. of Sewan village on Titve

Stream.

$2^{\circ} 14^{\prime} \mathrm{S} 138^{\circ} 46^{\prime} \mathrm{E}$ Alt: $10-100 \mathrm{~m}$

Habitat:

Primary lowland forest, canopy $10-60 \mathrm{~m}$ high; associates diverse, including Sterculia, Knema, Polyalthia, Pometia, Ziziphus.

Field Char: Understory tree $5 \mathrm{~m}$ tall, branching midway up, calyx green during anthesis, pink inside during fruition, corolla white, fruits maturing from green to purple.

Notes:

$\mathrm{NCI}$ voucher

McDonald \& Ismail 3701

24 May 1993

\section{HERBARIUM BOGORIENSE HARVARD UNIVERSITY HERBARIA}

\section{PLANTS OF INDONESIA}

Icacinaceae

Cat. \# 3702

\section{Gomphandra sp.}

Det. By: McDonald 1993

Locality:

New Guinea; Sarmi,

Coastal plain and Gunung Top, W of Sungai Tor, E

of Sungai Waske, vic. of Sewan village on Titve

Stream.

$2^{\circ} 14^{\prime}$ ' $138^{\circ} 46^{\prime}$ E Alt: $10-100 \mathrm{~m}$

Habitat:

Primary lowland forest, canopy $10-60 \mathrm{~m}$ high; associates diverse, including Sterculia, Knema, Polyalthia, Pometia, Ziziphus.

\section{Field Char:}

Large understory tree $8 \mathrm{~m}$ tall, branching above, new stem growth green, mature stems whitish; bole straight, cylindrical, bark pale gray, smooth; in bud, calyx pale green.

Notes:

NCI voucher

McDonald \& Ismail 3702 


\section{PLANTS OF INDONESIA}

Cat. \# 3703

Cleistanthes myrianthus (Hassk.) Kurz

Det. By: McDonald 1993

Locality:

New Guinea; Sarmi,

Coastal plain and Gunung Top, W of Sungai Tor, E

of Sungai Waske, vic. of Sewan village on Titve

Stream.

$2^{\circ} 14^{\prime} \mathrm{S} 138^{\circ} 46^{\prime} \mathrm{E}$ Alt: $10-100 \mathrm{~m}$

Habitat:

Primary lowland forest, canopy 10-60 m high; associates diverse, including Sterculia, Knema, Polyalthia, Pometia, Ziziphus.

Field Char:

Medium sized canopy tree, $12 \mathrm{~m}$ tall, branching

midway up, bole cylindrical, bark gray-brown, girdled with dark lichens, leaf undersurface silvercoppery.

Notes:

$\mathrm{NCI}$ voucher

McDonald \& Ismail $3703 \quad 24$ May 1993

\section{HERBARIUM BOGORIENSE \\ HARVARD UNIVERSITY HERBARIA}

\section{PLANTS OF INDONESIA}

Myristicaceae

Cat. \# 3704

Endocomia macrocoma (Miq.) De Wild spp. prainii (King) De Wilde

Det. By: McDonald 1993

Locality:

New Guinea; Sarmi,

Coastal plain and Gunung Top, W of Sungai Tor, E

of Sungai Waske, vic. of Sewan village on Titve

Stream.

$2^{\circ} 14^{\prime} \mathrm{S} 138^{\circ} 46^{\prime}$ E Alt: $10-100 \mathrm{~m}$

Habitat:

Primary lowland forest, canopy $10-60 \mathrm{~m}$ high; associates diverse, including Sterculia, Knema, Polyalthia, Pometia, Ziziphus.

Field Char:

Canopy tree, $30 \mathrm{~m}$ tall; bole straight, cylindrical, 30 $\mathrm{cm}$ diam, lateral branches whorled, the foliage persistent on the tips of these; bark whitish, smooth; corolla maroon, peduncles light green.

McDonald \& Ismail 3704

25 May 1993

HERBARIUM BOGORIENSE HARVARD UNIVERSITY HERBARIA 


\section{PLANTS OF INDONESIA}

Piperaceae

Cat. \# 3705

Piper arborescens Roxb.

Det. By: McDonald 1993

Locality:

New Guinea; Sarmi,

Coastal plain and Gunung Top, W of Sungai Tor, E

of Sungai Waske, vic. of Sewan village on Titve

Stream.

$2^{\circ} 14^{\prime} \mathrm{S} 138^{\circ} 46^{\prime} \mathrm{E}$ Alt: $10-100 \mathrm{~m}$

Habitat:

Primary lowland forest, canopy $10-60 \mathrm{~m}$ high; associates diverse, including Sterculia, Knema, Polyalthia, Pometia, Ziziphus.

Field Char:

Riparian liana, branching prodigiously, $25 \mathrm{~m}$ long; basal stem $5 \mathrm{~cm}$ diam; bark covered with whitish lichen.

McDonald \& Ismail 3705 25 May 1993

HERBARIUM BOGORIENSE HARVARD UNIVERSITY HERBARIA

\section{PLANTS OF INDONESIA}

Fabaceae

Cat. \# 3706

Pongamia cf. pinnata (L.) Pierre

Det. By: McDonald 1993

Locality:

New Guinea; Sarmi,

Coastal plain and Gunung Top, W of Sungai Tor, E of Sungai Waske, vic. of Sewan village on Titve

Stream.

$2^{\circ} 14^{\prime} \mathrm{S} 138^{\circ} 46^{\prime}$ 'E Alt: $10-100 \mathrm{~m}$

Habitat:

Primary lowland forest, canopy $10-60 \mathrm{~m}$ high; associates diverse, including Sterculia, Knema, Polyalthia, Pometia, Ziziphus.

Field Char:

Small canopy tree $13 \mathrm{~m}$ tall, branching midway up; bole contoured, $40 \mathrm{~cm}$ diam; foliage dark green, dense; immature fruits light green.

Notes:

$\mathrm{NCI}$ voucher

McDonald \& Ismail 3706

25 May 1993

HERBARIUM BOGORIENSE

HARVARD UNIVERSITY HERBARIA 


\section{PLANTS OF INDONESIA}

Cat. \# 3707

Neubergia sp. nov.

Det. By: McDonald 1993

Locality:

New Guinea; Sarmi,

Coastal plain and Gunung Top, W of Sungai Tor, E

of Sungai Waske, vic. of Sewan village on Titve

Stream.

$2^{\circ} 14^{\prime}$ 'S $138^{\circ} 46^{\prime}$ E Alt: $10-100 \mathrm{~m}$

Habitat:

Primary lowland forest, canopy $10-60 \mathrm{~m}$ high; associates diverse, including Sterculia, Knema, Polyalthia, Pometia, Ziziphus.

Field Char:

Understory tree $5 \mathrm{~m}$ tall, branching above and below; basal stem $5 \mathrm{~cm}$ diam; foliage dark green; calyx and corolla white, ripe fruits bright orange.

Notes:

NCI voucher

McDonald \& Ismail 3707

25 May 1993

HERBARIUM BOGORIENSE HARVARD UNIVERSITY HERBARIA

\section{PLANTS OF INDONESIA}

Moraceae

Cat. \# 3708

\section{Ficus aff. conocephalifolia}

Det. By: McDonald 1993

Locality:

New Guinea; Sarmi,

Coastal plain and Gunung Top, W of Sungai Tor, E

of Sungai Waske, vic. of Sewan village on Titve

Stream.

$2^{\circ} 14^{\prime} \mathrm{S} 138^{\circ} 46^{\prime} \mathrm{E}$ Alt: $10-100 \mathrm{~m}$

Habitat:

Primary lowland forest, canopy $10-60 \mathrm{~m}$ high;

associates diverse, including Sterculia, Knema,

Polyalthia, Pometia, Ziziphus.

Field Char:

Shrubby, riparian tree, branching profusely from base, basal stems to $4 \mathrm{~cm}$ diam, leaves clustered at stem apex; figs red, sweet and mucilaginous within, bracteate and bearing urticating hairs externally.

Notes:

$\mathrm{NCI}$ voucher

McDonald \& Ismail 3708

HERBARIUM BOGORIENSE HARVARD UNIVERSITY HERBARIA 


\section{PLANTS OF INDONESIA}

Cat. \# 3709

\section{Erythrospermum?}

Det. By: McDonald 1993

Locality:

New Guinea; Sarmi,

Coastal plain and Gunung Top, W of Sungai Tor, E of Sungai Waske, vic. of Sewan village on Titve

Stream.

$2^{\circ} 14^{\prime} \mathrm{S} 138^{\circ} 46^{\prime} \mathrm{E}$ Alt: $10-100 \mathrm{~m}$

Habitat:

Primary lowland forest, canopy 10-60 m high; associates diverse, including Sterculia, Knema, Polyalthia, Pometia, Ziziphus.

Field Char:

Midstory tree, $18 \mathrm{~m}$ tall, branching above; bole straight, cylindrical, $30 \mathrm{~cm}$ diam; bark gray, rough, immature fruits pale green.

Notes:

$\mathrm{NCI}$ voucher

McDonald \& Ismail 3709

25 May 1993

\section{HERBARIUM BOGORIENSE HARVARD UNIVERSITY HERBARIA}

\section{PLANTS OF INDONESIA}

Simaroubaceae

Cat. \# 3710

Quassia indica (Gaertn.) Nooteb.

Det. By: McDonald 1993

Locality:

New Guinea; Sarmi,

Coastal plain and Gunung Top, W of Sungai Tor, E of Sungai Waske, vic. of Sewan village on Titve

Stream.

$2^{\circ} 14$ 'S $138^{\circ} 46$ 'E Alt: 10 $100 \mathrm{~m}$

Habitat:

Primary lowland forest, canopy 10-60 m high; associates diverse, including Sterculia, Knema, Polyalthia, Pometia, Ziziphus.

\section{Field Char:}

Understory tree $5 \mathrm{~m}$ tall, branching above and below, lateral branches much contorted; bark red-tan, smooth; leaves dark green above, pale green below; flowers in bud, petals pale green, stamen filaments reddish, anthers yellow, style reddish, carpels 3 or 4 . Notes:

$\mathrm{NCI}$ voucher

McDonald \& Ismail 3710 


\section{PLANTS OF INDONESIA}

Myristicaceae

Cat. \# 3711

\section{Myristica concinna Sinclair}

Det. By: McDonald 1993

Locality:

New Guinea; Sarmi,

Coastal plain and Gunung Top, W of Sungai Tor, E of Sungai Waske, vic. of Sewan village on Titve

Stream.

$2^{\circ} 14^{\prime} \mathrm{S} 138^{\circ} 46^{\prime} \mathrm{E}$ Alt: $10-100 \mathrm{~m}$

Habitat:

Primary lowland forest, canopy 10-60 m high; associates diverse, including Sterculia, Knema, Polyalthia, Pometia, Ziziphus.

Field Char:

Understory tree $7 \mathrm{~m}$ tall, branching above and below, lateral branches perpendicular to trunk; bole straight, cylindrical, $10 \mathrm{~cm}$ diam, bark smooth; flowers urceolate, yellow.

Notes:

NCI voucher

McDonald \& Ismail 3711

25 May 1993

\section{HERBARIUM BOGORIENSE HARVARD UNIVERSITY HERBARIA}

\section{PLANTS OF INDONESIA}

Rubiaceae

Dolicholobium oxylobum (K. Sch.) Laut.

Det. By: McDonald 1993

Locality:

New Guinea; Sarmi,

Coastal plain and Gunung Top, W of Sungai Tor, E

of Sungai Waske, vic. of Sewan village on Titve

Stream.

$2^{\circ} 14^{\prime}$ 'S $138^{\circ} 46^{\prime}$ E Alt: $10-100 \mathrm{~m}$

Habitat:

Primary lowland forest, canopy 10-60 m high; associates diverse, including Sterculia, Knema, Polyalthia, Pometia, Ziziphus.

Field Char:

Understory riparian tree $4 \mathrm{~m}$ tall, bole straight, $8 \mathrm{~cm}$ diam; bark brown, flaky; corolla white, throat yellow, segments 5 or 6 , inflorescence bracts yellowciliate.

Notes:

$\mathrm{NCI}$ voucher

McDonald \& Ismail $3712 \quad 25$ May 1993 


\section{PLANTS OF INDONESIA}

Meliaceae

Cat. \# 3713

Dysoxylum arborescens (Bl.) Miq.

Det. By: McDonald 1993

Locality:

New Guinea; Sarmi,

Coastal plain and Gunung Top, W of Sungai Tor, E of Sungai Waske, vic. of Sewan village on Titve

Stream.

$2^{\circ} 14^{\prime} \mathrm{S} 138^{\circ} 46^{\prime} \mathrm{E}$ Alt: $10-100 \mathrm{~m}$

Habitat:

Primary lowland forest, canopy 10-60 m high; associates diverse, including Sterculia, Knema, Polyalthia, Pometia, Ziziphus.

Field Char:

Midstory tree, $12 \mathrm{~m}$ tall, riparian, branching midway up; bole straight, cylindrical, $13 \mathrm{~cm}$ diam; bark tan, rough; fruits sparse, red.

Notes:

$\mathrm{NCI}$ voucher

McDonald \& Ismail 3713

25 May 1993

HERBARIUM BOGORIENSE

HARVARD UNIVERSITY HERBARIA

\section{PLANTS OF INDONESIA}

Cat. \# 3714

Decasperma sp.

Det. By: McDonald 1993

Locality:

New Guinea; Sarmi,

Coastal plain and Gunung Top, W of Sungai Tor, E

of Sungai Waske, vic. of Sewan village on Titve

Stream.

$2^{\circ} 14^{\prime} \mathrm{S} 138^{\circ} 46^{\prime} \mathrm{E}$ Alt: $10-100 \mathrm{~m}$

Habitat:

Primary lowland forest, canopy 10-60 m high; associates diverse, including Sterculia, Knema, Polyalthia, Pometia, Ziziphus.

Field Char:

Occasional midstory tree, $20 \mathrm{~m}$ tall; bole straight, cylindrical; bark pale gray, smooth; leaves pale green below, dark green above; calyx pale green, stamens white, ca. $1 \mathrm{~cm}$ long.

Notes:

NCI voucher

McDonald \& Ismail 3714

25 May 1993

HERBARIUM BOGORIENSE HARVARD UNIVERSITY HERBARIA 


\section{PLANTS OF INDONESIA}

Meliaceae

Cat. \# 3715

Aglaia goebeliana Warb.

Det. By: McDonald 1993

Locality:

New Guinea; Sarmi,

Coastal plain and Gunung Top, W of Sungai Tor, E

of Sungai Waske, vic. of Sewan village on Titve

Stream.

$2^{\circ} 4^{\prime}$ S $138^{\circ} 46^{\prime} \mathrm{E}$ Alt: $10-100 \mathrm{~m}$

Habitat:

Primary lowland forest, canopy 10-60 m high; associates diverse, including Sterculia, Knema, Polyalthia, Pometia, Ziziphus.

Field Char:

Understory tree $7 \mathrm{~m}$ tall; bole straight, cylindrical, 8 $\mathrm{cm}$ diam; bark tan, narrowly grooved longitudinally, fibrous; leaflets dark green above, pale green below; inflorescence coppery-green.

McDonald \& Ismail 3715

26 May 1993

\section{HERBARIUM BOGORIENSE HARVARD UNIVERSITY HERBARIA}

\section{PLANTS OF INDONESIA}

Cat. \# 3716

Kingiodendron cf. alternifolia

Det. By: McDonald 1993

Locality:

New Guinea; Sarmi,

Coastal plain and Gunung Top, W of Sungai Tor, E of Sungai Waske, vic. of Sewan village on Titve

Stream.

$2^{\circ} 4^{\prime} \mathrm{S} 138^{\circ} 46^{\prime} \mathrm{E}$ Alt: $10-100 \mathrm{~m}$

Habitat:

Primary lowland forest, canopy 10-60 m high; associates diverse, including Sterculia, Knema, Polyalthia, Pometia, Ziziphus.

Field Char:

Midstory tree $10 \mathrm{~m}$ tall; bole straight, cylindrical, 20 cm diam; inner bark red, outer tan; flowers abundant, pale green.

McDonald \& Ismail 3716

26 May 1993

HERBARIUM BOGORIENSE HARVARD UNIVERSITY HERBARIA 


\title{
PLANTS OF INDONESIA
}

Euphorbiaceae

Cat. \# 3717

Pimelodendron amboinicum Kassk.

Det. By: McDonald 1993

Locality:

New Guinea; Sarmi,

Coastal plain and Gunung Top, W of Sungai Tor, E of Sungai Waske, vic. of Sewan village on Titve

Stream.

$2^{\circ} 4^{\prime} \mathrm{S} 138^{\circ} 46^{\prime} \mathrm{E}$ Alt: $10-100 \mathrm{~m}$

Habitat:

Primary lowland forest, canopy 10-60 m high; associates diverse, including Sterculia, Knema, Polyalthia, Pometia, Ziziphus.

Field Char:

Canopy tree $35 \mathrm{~m}$ tall, bole much contoured and grooved, $45 \mathrm{~cm}$ diam.; dark brown, latex white; foliage dense, dark green; calyx green, anthers many, bright yellow.

McDonald \& Ismail 3717

26 May 1993

\author{
HERBARIUM BOGORIENSE \\ HARVARD UNIVERSITY HERBARIA
}

\section{PLANTS OF INDONESIA}

Dipterocarpaceae

Cat. \# 3718

Vatica rassak (Korth.) Bl.

Det. By: McDonald 1993

Locality:

New Guinea; Sarmi,

Coastal plain and Gunung Top, W of Sungai Tor, E

of Sungai Waske, vic. of Sewan village on Titve

Stream.

$2^{\circ} 4^{\prime} \mathrm{S} 138^{\circ} 46^{\prime} \mathrm{E}$ Alt: $10-100 \mathrm{~m}$

Habitat:

Primary lowland forest, canopy 10-60 m high; associates diverse, including Sterculia, Knema, Polyalthia, Pometia, Ziziphus.

Field Char:

Canopy tree $35 \mathrm{~m}$ tall; bole straight, cylindrical, 40 $\mathrm{cm}$ diam.; bark pale gray, smooth, girdled by narrow, horizontal ridges; branching above; sepals pale green, carolla cream, petals yellowish at base, very aromtaic (like Garcinia)

McDonald \& Ismail 3718

26 May 1993

HERBARIUM BOGORIENSE HARVARD UNIVERSITY HERBARIA 


\title{
PLANTS OF INDONESIA
}

Ebenaceae

Cat. \# 3719

Diospyros celebica Bakh.

Det. By: McDonald 1993

Locality:

New Guinea; Sarmi,

Coastal plain and Gunung Top, W of Sungai Tor, E

of Sungai Waske, vic. of Sewan village on Titve

Stream.

$2^{\circ} 4^{\prime} \mathrm{S} 138^{\circ} 46^{\prime} \mathrm{E}$ Alt: $10-100 \mathrm{~m}$

Habitat:

Primary lowland forest, canopy $10-60 \mathrm{~m}$ high; associates diverse, including Sterculia, Knema, Polyalthia, Pometia, Ziziphus.

Field Char:

Canopy tree, $20 \mathrm{~m}$ tall; branching midway up; bole straight, contoured, $25 \mathrm{~cm}$ diam., slightly buttressed at base; bark grayish, ridged and somewhat flaky; fruit orange, 5-6 cm diam.

McDonald \& Ismail 3719

26 May 1993

\author{
HERBARIUM BOGORIENSE \\ HARVARD UNIVERSITY HERBARIA
}

\section{PLANTS OF INDONESIA}

Moraceae

Cat. \# 3720

Ficus tinctoria Forst. $f$.

Det. By: McDonald 1993

Locality:

New Guinea; Sarmi,

Coastal plain and Gunung Top, W of Sungai Tor, E

of Sungai Waske, vic. of Sewan village on Titve

Stream.

$2^{\circ} 4^{\prime} \mathrm{S} 138^{\circ} 46^{\prime} \mathrm{E}$ Alt: $10-100 \mathrm{~m}$

Habitat:

Primary lowland forest, canopy 10-60 m high; associates diverse, including Sterculia, Knema, Polyalthia, Pometia, Ziziphus.

Field Char:

Common riparian tree overhanging stream, $10 \mathrm{~m}$ tall, branching 5 times from base, boles $5-15 \mathrm{~cm}$ diam.; bark greenish-tan, smooth; figs pale green, orange upon ripening.

McDonald \& Ismail 3720

26 May 1993

\section{HERBARIUM BOGORIENSE} HAR VARD UNIVERSITY HERBARIA 


\section{PLANTS OF INDONESIA}

Meliaceae

Cat. \# 3721

Chisocheton lasiocarpus (Miq.) Val.

Det. By: McDonald 1993

Locality:

New Guinea; Sarmi,

Coastal plain and Gunung Top, W of Sungai Tor, E of Sungai Waske, vic. of Sewan village on Titve

Stream.

$2^{\circ} 4^{\prime} \mathrm{S} 138^{\circ} 46^{\prime} \mathrm{E}$ Alt: $10-100 \mathrm{~m}$

Habitat:

Primary lowland forest, canopy 10-60 m high; associates diverse, including Sterculia, Knema, Polyalthia, Pometia, Ziziphus.

Field Char:

Understory tree $8 \mathrm{~m}$ tall, branching sparingly above and below, trunk straight, cylindrical, $10 \mathrm{~cm}$ diam.; bark dark brown, somewhat rough; stems hollow, inhabited by aggressive ants; flowers whitish with a tinge of pink, petals 3 , reflexed, staminal column urceolate.

McDonald \& Ismail 3721

26 May 1993

\section{HERBARIUM BOGORIENSE HARVARD UNIVERSITY HERBARIA}

\section{PLANTS OF INDONESIA}

Moraceae

Cat. \# 3722

Ficus cf. wassa Roxb.

Det. By: McDonald 1993

Locality:

New Guinea; Sarmi,

Coastal plain and Gunung Top, W of Sungai Tor, E of Sungai Waske, vic. of Sewan village on Titve Stream.

$2^{\circ} 4$ 'S $138^{\circ} 46^{\prime}$ E Alt: $10-100 \mathrm{~m}$

Habitat:

Primary lowland forest, canopy 10-60 m high; associates diverse, including Sterculia, Knema, Polyalthia, Pometia, Ziziphus.

Field Char:

Midstory riparian tree $12 \mathrm{~m}$ tall, branching above and below; bole straight, cylindrical, $12 \mathrm{~cm}$ diam.; bark brownish, smooth; figs cauliforous along trunk, sparing in leaf axils, small and greenish during maturation, ants building nests in fig clusters on trunk. 


\title{
PLANTS OF INDONESIA
}

Myristicaceae

Cat. \# 3723

Myristica fatua Houtt. var. papuana $\mathrm{Mg}$

Det. By: McDonald 1993

Locality:

New Guinea; Sarmi,

Coastal plain and Gunung Top, W of Sungai Tor, E of Sungai Waske, vic. of Sewan village on Titve

Stream.

$2^{\circ} 4^{\prime} \mathrm{S} 138^{\circ} 46^{\prime} \mathrm{E}$ Alt: $10-100 \mathrm{~m}$

Habitat:

Primary lowland forest, canopy 10-60 m high; associates diverse, including Sterculia, Knema, Polyalthia, Pometia, Ziziphus.

Field Char:

Canopy tree $35 \mathrm{~cm}$ tall; bole straight, cylindrical, 30 $\mathrm{cm}$ diam.; bark grayish, fissured; lateral branches curving downward; foliage light green-coppery; fruits copper.

McDonald \& Ismail 3723

26 May 1993

\author{
HERIBARIUM BOGORIENSE \\ HARVARD UNIVERSITY HERBARIA
}

\section{PLANTS OF INDONESIA}

Arecaceae

Cat. \# 3724

Caryota rumphiana Mart.

Det. By: McDonald 1993

Locality:

New Guinea; Sarmi,

Coastal plain and Gunung Top, W of Sungai Tor, E

of Sungai Waske, vic. of Sewan village on Titve

Stream.

$2^{\circ} 4$ 'S $138^{\circ} 46^{\prime}$ E Alt: $10-100 \mathrm{~m}$

Habitat:

Primary lowland forest, canopy 10-60 m high; associates diverse, including Sterculia, Knema, Polyalthia, Pometia, Ziziphus.

Field Char:

Uncommon, midstory or canopy palm, $20 \mathrm{~m}$ tall, trunk $25 \mathrm{~cm}$ diam. at base, internodes ca. $0.5 \mathrm{~m}$, fronds $8 \mathrm{~m}$ long, $2 \mathrm{~m}$ wide, leaf bases $1.5 \mathrm{~m}$ long, $25 \mathrm{~cm}$ wide; rachises of inflorescence many hundreds, $1.5-2 \mathrm{~m}$ long during fruition; berries maroon at maturity.

McDonald \& Ismail 3724 


\section{PLANTS OF INDONESIA}

Ulmaceae

Cat. \# 3725

Gironniera celtidifolia Gaudich.

Det. By: McDonald 1993

Locality:

New Guinea; Sarmi,

Coastal plain and Gunung Top, W of Sungai Tor, E

of Sungai Waske, vic. of Sewan village on Titve

Stream.

$2^{\circ} 14^{\prime} \mathrm{S} 138^{\circ} 46^{\prime} \mathrm{E}$ Alt: $10-100 \mathrm{~m}$

Habitat:

Primary lowland forest, canopy 10-60 m high; associates diverse, including Sterculia, Knema, Polyalthia, Pometia, Ziziphus.

Field Char:

Understory tree $9 \mathrm{~m}$ tall; bole staight, cylindrical, 14

$\mathrm{cm}$ diam., branching above; immature fruits green.

McDonald \& Ismail 3725

27 May 1993

\section{HERBARIUM BOGORIENSE \\ HARVARD UNIVERSITY HERBARIA}

\section{PLANTS OF INDONESIA}

Ulmaceae

Cat. \# 3726

Gironniera rhamnifolia $\boldsymbol{B l}$.

Det. By: McDonald 1993

Locality:

New Guinea; Sarmi,

Coastal plain and Gunung Top, W of Sungai Tor, E

of Sungai Waske, vic. of Sewan village on Titve

Stream.

$2^{\circ} 14^{\prime} \mathrm{S} 138^{\circ} 46^{\prime} \mathrm{E}$ Alt: $10-100 \mathrm{~m}$

Habitat:

Primary lowland forest, canopy $10-60 \mathrm{~m}$ high; associates diverse, including Sterculia, Knema, Polyalthia, Pometia, Ziziphus.

Field Char:

Midstory tree $13 \mathrm{~m}$ tall; bole straight, bark pale tan, somewhat rough; fruits maturing from green to orange brown, hard-fleshy.

McDonald \& Ismail 3726

27 May 1993

HERBARIUM BOGORIENSE HARVARD UNIVERSITY HERBARIA 


\section{PLANTS OF INDONESIA}

Annonaceae

Cat. \# 3727

\section{Annonaceae}

Det. By: McDonald 1993

Locality:

New Guinea; Sarmi,

Coastal plain and Gunung Top, W of Sungai Tor, E of Sungai Waske, vic. of Sewan village on Titve

Stream.

$2^{\circ} 14^{\prime} \mathrm{S} 138^{\circ} 46^{\prime} \mathrm{E}$ Alt: $10-100 \mathrm{~m}$

Habitat:

Primary lowland forest, canopy 10-60 m high; associates diverse, including Sterculia, Knema, Polyalthia, Pometia, Ziziphus.

Field Char:

Midstory tree $13 \mathrm{~m}$ tall; bole straight, cylindrical, 10 $\mathrm{cm}$. diam., branching midway up; bark pale gray, smooth; lateral branches perpendicular to trunk; immature fruits green, cauliflorous or axillary.

McDonald \& Ismail 3727

27 May 1993

\section{HERBARIUM BOGORIENSE HARVARD UNIVERSITY HERBARIA}

\section{PLANTS OF INDONESIA}

Rubiaceae

Cat. \# 3728

Ixora cf. leptopus Val.

Det. By: McDonald 1993

Locality:

New Guinea; Sarmi,

Coastal plain and Gunung Top, W of Sungai Tor, E

of Sungai Waske, vic. of Sewan village on Titve

Stream.

$2^{\circ} 14^{\prime} \mathrm{S} 138^{\circ} 46^{\prime} \mathrm{E}$ Alt: $10-100 \mathrm{~m}$

Habitat:

Primary lowland forest, canopy 10-60 m high; associates diverse, including Sterculia, Knema, Polyalthia, Pometia, Ziziphus.

Field Char:

Understory tree $8 \mathrm{~m}$ tall, branching profusely above; trunk straight, cylindrical, $8 \mathrm{~cm}$ diam.; bark pale gray; calyx green, corolla pale cream during anthesis; limbs and stamens reflexed; styles and stigma reddish; berry reddish.

Mcdonald and Ismail 3728

27 May 1993 


\section{PLANTS OF INDONESIA}

Sapindaceae

Cat. \# 3729

Sapindaceae

Det. By: McDonald 1993

Locality:

New Guinea; Sarmi,

Coastal plain and Gunung Top, W of Sungai Tor, E of Sungai Waske, vic. of Sewan village on Titve

Stream.

$2^{\circ} 14^{\prime} \mathrm{S} 138^{\circ} 46^{\prime} \mathrm{E}$ Alt: $10-100 \mathrm{~m}$

Habitat:

Primary lowland forest, canopy 10-60 m high; associates diverse, including Sterculia, Knema, Polyalthia, Pometia, Ziziphus.

Field Char:

Midstory tree $18 \mathrm{~m}$ tall; bole somewhat crooked, 20 $\mathrm{cm}$ diam., bark pale gray, rough; fruits peachypuberulent, sparing.

McDonald and Ismail 3729

27 May 1993

HERBARIUM BOGORIENSE HARVARD UNIVERSITY HERBARIA

\section{PLANTS OF INDONESIA}

Piperaceae

Cat. \# 3730

Piper cf. decumanum (Rumph) $L$.

Det. By: McDonald 1993

Locality:

New Guinea; Sarmi,

Coastal plain and Gunung Top, W of Sungai Tor, E of Sungai Waske, vic. of Sewan village on Titve

Stream.

$2^{\circ} 14^{\prime} \mathrm{S} 138^{\circ} 46^{\prime} \mathrm{E}$ Alt: $10-100 \mathrm{~m}$

Habitat:

Primary lowland forest, canopy 10-60 m high; associates diverse, including Sterculia, Knema, Polyalthia, Pometia, Ziziphus.

Field Char:

Liana, to $10 \mathrm{~m}$ long, adhering to tree trunks by branching, adventitious roots; foliage dark green, leaves aromatic; inflorescence ca. $75 \mathrm{~cm}$ long, yellowish-green, pungent when green.

McDonald and Ismail 3730

HERBARIUM BOGORIENSE HARVARD UNIVERSITY HERBARIA 


\section{Icacinaceae}

Det. By: McDonald 1993

Locality:

New Guinea; Sarmi,

Coastal plain and Gunung Top, W of Sungai Tor, E

of Sungai Waske, vic. of Sewan village on Titve

Stream.

$2^{\circ} 14^{\prime} \mathrm{S} 138^{\circ} 46^{\prime} \mathrm{E}$ Alt: $10-100 \mathrm{~m}$

Habitat:

Primary lowland forest, canopy 10-60 m high; associates diverse, including Sterculia, Knema, Polyalthia, Pometia, Ziziphus.

Field Char:

Midstory tree $13 \mathrm{~m}$ tall; bole straight, somewhat contoured, $30 \mathrm{~cm}$ diam., branching above; calyx green, basally cupulate; fruit green, seed 1 , placentation apical.

McDonald and Ismail 3731

27 May 1993

\section{Icacinaceae}

Det. By: McDonald 1993

Locality:

New Guinea; Sarmi,

Coastal plain and Gunung Top, W of Sungai Tor, E of Sungai Waske, vic. of Sewan village on Titve Stream.

$2^{\circ} 14^{\prime} \mathrm{S} 138^{\circ} 46^{\prime} \mathrm{E}$ Alt: $10-100 \mathrm{~m}$

\section{Habitat:}

Primary lowland forest, canopy 10-60 m high; associates diverse, including Sterculia, Knema, Polyaithia, Pometia, Ziziphus.

Field Char: Midstory tree $13 \mathrm{~m}$ tail; bole straight, somewhat contoured, $30 \mathrm{~cm}$ diam., branching above; calyx green, basally cupulate; fruit green, seed 1 , placentation apical.

McDonald and Ismail 3731

27 May 1993

\section{HARVARD UNIVERSITY HERBARIA}

\section{PLANTS OF INDONESIA}

Icacinaceae

Cat. \# 3732

\section{Medusanthera laxiflora (Miers) Howard}

Det. By: McDonald 1993

\section{Locality:}

New Guinea; Sarmi,

Coastal plain and Gunung Top, W of Sungai Tor, E of Sungai Waske, vic. of Sewan village on Titve Stream.

$2^{\circ} 14^{\prime} \mathrm{S} 138^{\circ} 46^{\prime} \mathrm{E}$ Alt: $10-100 \mathrm{~m}$

\section{Habitat:}

Primary lowland forest, canopy $10-60 \mathrm{~m}$ high; associates diverse, including Sterculia, Knema, Polyalthia, Pometia, Ziziphus.

Field Char:

Midstory tree $12 \mathrm{~m}$ tall, branching midway up; bole straight, cylindrical, $12 \mathrm{~cm}$ diam.; bark tan-gray, rough; lateral branches perpendicular to trunk; foliage glossy; fruit curved, dorsal surface blackpurple, 3 ridged, ventral surface cream.

McDonald and Ismail 3732 


\section{PLANTS OF INDONESIA}

Simaroubaceae

Cat. \# 3733

Picrasma javanica Bl.

Det. By: McDonald 1993

Locality:

New Guinea; Sarmi,

Near Sewan village, $W$ of Sungai Tor, E of Sungai

Waske, on slopes of Gunung Top

$2^{\circ} 4^{\prime} 138^{\circ} 46^{\prime}$ Alt: $200-300 \mathrm{~m}$

Habitat:

Primary, low-montane forest, canopy $20-50 \mathrm{~m}$ tall; associates include: Myristica, Mammea, Semecarpus, Chionanthus, Gonocaryum.

Field Char:

Midstory tree $14 \mathrm{~m}$ tall, branching above; bole

straight, slightly contoured, $15 \mathrm{~cm}$ diam.; immature

fruits light-green, 2-3 per flower.

McDonald and Ismail 3733

27 May 1993

\section{HERBARIUM BOGORIENSE \\ HARVARD UNIVERSITY HERBARIA}

\section{PLANTS OF INDONESIA}

Rubiaceae

Cat. \# 3734

Psychotria damasiana Sohmer

Det. By: McDonald 1993

Locality:

New Guinea; Sarmi,

Near Sewan village, W of Sungai Tor, E of Sungai

Waske, on slopes of Gunung Top

$2^{\circ} 4^{\prime} \quad 138^{\circ} 46^{\prime}$ Alt: $200-300 \mathrm{~m}$

Habitat:

Primary, low-montane forest, canopy $20-50 \mathrm{~m}$ tall; associates include: Myristica, Mammea, Semecarpus, Chionanthus, Gonocaryum.

Field Char:

Understory tree $4 \mathrm{~m}$ tall; bole straight, cylindrical, 8 cm diam.; bark pale gray, smooth, corollas urceolate, tube cream, lobes reflexed, pale green; berries reddish at maturity.

McDonald and Ismail 3734

27 May 1993

HERBARIUM BOGORIENSE HARVARD UNIVERSITY HERBARIA 


\section{PLANTS OF INDONESIA}

Myristicaceae

Cat. \# 3735

Myristica sp.

Det. By: McDonald 1993

Locality:

New Guinea; Sarmi,

Upper ridges of small mountain emerging from

coastal plain; W of Sungai Tor, E of Sungai Waske, near Sewan vill.

$2^{\circ} 4^{\prime} \quad 138^{\circ} 46^{\prime}$ Alt: $200-300 \mathrm{~m}$

Habitat:

Primary, low-montane forest, canopy 20-50 m tall; associates include: Myristica, Mammea, Semecarpus, Chionanthus, Gonocaryum.

Field Char:

Canopy tree $30 \mathrm{~m}$ tall, branching above, bole thin, straight, cylindrical, $25 \mathrm{~cm}$ diam.; bark grayish, fissures longitudinal; flower buds green-coppery

McDonald \& Ismail 3735

28 May 1993

HERBARIUM BOGORIENSE

HARVARD UNIVERSITY HERBARIA

\section{PLANTS OF INDONESIA}

Rubiaceae

Cat. \# 3736

Canthium auantiacum Merr. \& Perry

Det. By: McDonald 1993

Locality:

New Guinea; Sarmi,

Upper ridges of small mountain emerging from

coastal plain; W of Sungai Tor, E of Sungai Waske, near Sewan vill.

$2^{\circ} 4^{\prime} 138^{\circ} 46^{\prime}$ Alt: $200-300 \mathrm{~m}$

Habitat:

Primary, low-montane forest, canopy $20-50 \mathrm{~m}$ tall; associates include: Myristica, Mammea, Semecarpus, Chionanthus, Gonocaryum.

Field Char:

Midstory tree $20 \mathrm{~m}$ tall, branching midway up; bole straight, cylindrical, $28 \mathrm{~cm}$ diam., wood hard; bark reddish, rough; fruit bright orange, smells of sewage and sweetness.

McDonald \& Ismail 3736

28 May 1993

HERBARIUM BOGORIENSE HARVARD UNIVERSITY HERBARIA 


\section{PLANTS OF INDONESIA}

Clusiaceae

Cat. \# 3737

Mammea novoguineensis

Det. By: McDonald 1993

Locality:

New Guinea; Sarmi,

Upper ridges of small mountain emerging from

coastal plain; W of Sungai Tor, E of Sungai Waske, near Sewan vill.

$2^{\circ} 4^{\prime} 138^{\circ} 46^{\prime}$ Alt: $200-300 \mathrm{~m}$

Habitat:

Primary, low-montane forest, canopy $20-50 \mathrm{~m}$ tall; associates include: Myristica, Mammea, Semecarpus, Chionanthus, Gonocaryum.

Field Char:

Canopy tree $22 \mathrm{~m}$, branching above, bole straight, cylindrical, $35 \mathrm{~m}$ diam.; inner bark reddish, outer grayish, latex pale yellow, sparing; fruits abundant, cauliflorous on lateral branches, brownish.

McDonald \& Ismail 3737

28 May 1993

\section{HERBARIUM BOGORIENSE HARVARD UNIVERSITY HERBARIA}

\section{PLANTS OF INDONESIA}

Rubiaceae

Cat. \# 3738

Timonius $s p$.

Det. By: McDonald 1993

Locality:

New Guinea; Sarmi,

Upper ridges of small mountain emerging from

coastal plain; W of Sungai Tor, E of Sungai Waske,

near Sewan vill.

$2^{\circ} 4^{\prime} 138^{\circ} 46^{\prime}$ Alt: $200-300 \mathrm{~m}$

Habitat:

Primary, low-montane forest, canopy $20-50 \mathrm{~m}$ tall; associates include: Myristica, Mammea, Semecarpus, Chionanthus, Gonocaryum.

Field Char:

Midstory tree $18 \mathrm{~m}$ tall, branching above; bole contoured, somwehat crooked; bark brownish, flaky; calyx green, corolla cream or pale yellow, limb segments 4 or 5 , reflexed.

McDonald \& Ismail 3738

28 May 1993

HERBARIUM BOGORIENSE HARVARD UNIVERSITY HERBARIA 


\section{PLANTS OF INDONESIA}

Anacardiaceae

Cat. \# 3739

Semecarpus bracteatus Laut.

Det. By: McDonald 1993

Locality:

New Guinea; Sarmi,

Upper ridges of small mountain emerging from

coastal plain; W of Sungai Tor, E of Sungai Waske, near Sewan vill.

$2^{\circ} 4^{\prime} \quad 138^{\circ} 46^{\prime}$ Alt: $200-300 \mathrm{~m}$

Habitat:

Primary, low-montane forest, canopy 20-50 m tall; associates include: Myristica, Mammea, Semecarpus, Chionanthus, Gonocaryum.

Field Char:

Midstory tree $18 \mathrm{~m}$ tall, branching above, bole straight, cylindrical, $20 \mathrm{~cm}$ diam.; bark pale tan, narrowly furrowed, latex black; foliage clustered at stem apex, leaves 0.5-1 m long; flowers pale green, stamens and petals 5 .

McDonald \& Ismail 3739

28 May 1993

\section{HERBARIUM BOGORIENSE HARVARD UNIVERSITY HERBARIA}

\section{PLANTS OF INDONESIA}

Apocynaceae

Cat. \# 3740

\section{Lepiniopsis ternatensis Val.}

Det. By: McDonald 1993

Locality:

New Guinea; Sarmi,

Upper ridges of small mountain emerging from coastal plain; W of Sungai Tor, E of Sungai Waske, near Sewan vill.

$2^{\circ} 4^{\prime} \quad 138^{\circ} 46^{\prime}$ Alt: $200-300 \mathrm{~m}$

Habitat:

Primary, low-montane forest, canopy $20-50 \mathrm{~m}$ tall; associates include: Myristica, Mammea, Semecarpus, Chionanthus, Gonocaryum.

Field Char:

Midstory tree $12 \mathrm{~m}$ tall, branching above; bole straight, cylindrical, $20 \mathrm{~cm}$ diam.; foliage dark green, lateral branches crooked; latex white, abundant; corolla white, limb segments twisted; immature fruits narrowly ellipsoid, green.

McDonald \& Ismail 3740 28 May 1993

HERBARIUM BOGORIENSE HARVARD UNIVERSITY HERBARIA 


\section{PLANTS OF INDONESIA}

Sterculiaceae

Cat. \# 3741

Sterculia macrophylla Vent.

Det. By: McDonald 1993

Locality:

New Guinea; Sarmi,

Upper ridges of small mountain emerging from

coastal plain; W of Sungai Tor, E of Sungai Waske, near Sewan vill.

$2^{\circ} 4^{\prime} 138^{\circ} 46^{\prime}$ Alt: $200-300 \mathrm{~m}$

Habitat:

Primary, low-montane forest, canopy $20-50 \mathrm{~m}$ tall; associates include: Myristica, Mammea, Semecarpus, Chionanthus, Gonocaryum.

Field Char:

Midstory tree $15 \mathrm{~m}$ tall, branching above; bole straight, cylindrical, $20 \mathrm{~cm}$ diam.; bark pale gray, smoothish; fruits 5-follicular, copper-pubescent.

McDonald \& Ismail $3741 \quad 28$ May 1993

\section{HERBARIUM BOGORIENSE \\ HARVARD UNIVERSITY HERBARIA}

\section{PLANTS OF INDONESIA}

Rubiaceae

Cat. \# 3742

Ixora cf. kochii Brem.

Det. By: McDonald 1993

Locality:

New Guinea; Sarmi,

Upper ridges of small mountain emerging from

coastal plain; W of Sungai Tor, E of Sungai Waske, near Sewan vill.

$2^{\circ} 4^{\prime} 138^{\circ} 46^{\prime}$ Alt: $200-300 \mathrm{~m}$

Habitat:

Primary, low-montane forest, canopy $20-50 \mathrm{~m}$ tall; associates include: Myristica, Mammea, Semecarpus, Chionanthus, Gonocaryum.

Field Char:

Midstory tree $11 \mathrm{~m}$ tall, branching above, bole straight, cylindrical, wood hard; bark pale gray, rough; foliage glossy; corolla white, turning yellowish, limb segment margins revolute during anthesis. 


\section{PLANTS OF INDONESIA}

Arecaceae

Cat. \# 3743

Sommieria elegans Becc.

Det. By: McDonald 1993

Locality:

New Guinea; Sarmi,

Upper ridges of small mountain emerging from

coastal plain; W of Sungai Tor, E of Sungai Waske, near Sewan vill.

$2^{\circ} 4^{\prime} 138^{\circ} 46^{\prime}$ Alt: $200-300 \mathrm{~m}$

Habitat:

Primary, low-montane forest, canopy $20-50 \mathrm{~m}$ tall; associates include: Myristica, Mammea, Semecarpus, Chionanthus, Gonocaryum.

Field Char:

Understory palm, 1-3 m tall, basal stem solitary, 2-4 $\mathrm{cm}$ diam., terete; foliage in rosette; fronds $1-1.5 \mathrm{~m}$ long, deeply bifid at apex, corrugate; flowers yellowbrown; berries cream with brown indumentum turning red at maturity.

McDonald \& Ismail 3743

28 May 1993

\section{HERBARIUM BOGORIENSE HARVARD UNIVERSITY HERBARIA}

\section{PLANTS OF INDONESIA}

Oleaceae

Cat. \# 3744

Chionanthus ramiflorus Roxb.

Det. By: McDonald 1993

Locality:

New Guinea; Sarmi,

Coastal plain and Gunung Top, W of Sungai Tor, E

of Sungai Waske, vic. of Sewan village on Titve

stream.

$2^{\circ} 4^{\prime}$ 'S $138^{\circ} 46^{\prime}$ E Alt: $10-100 \mathrm{~m}$

Habitat:

Primary lowland forest, canopy $10-60 \mathrm{~m}$ high; associates diverse, include: Sterculia, Knema, Polyathia, Pometia.

Field Char:

Canopy tree $24 \mathrm{~m}$ tall, branching midway up; bole straight, cylindrical, $25 \mathrm{~cm}$ diam.; bark pale gray, smoothish; flowers yellow-green, stamens 2 ; fruit green, pale-corky below.

McDonald \& Ismail 3744

29 May 1993

HERBARIUM BOGORIENSE HARVARD UNIVERSITY HERBARIA 


\section{PLANTS OF INDONESIA}

Cat. \# 3745

Ptychopixis philipinensis Croiz.

Det. By: McDonald 1993

Locality:

New Guinea; Sarmi,

Coastal plain and Gunung Top, W of Sungai Tor, E of Sungai Waske, vic. of Sewan village on Titve stream.

$2^{\circ} 4^{\prime} \mathrm{S} 138^{\circ} 46^{\prime} \mathrm{E}$ Alt: $10-100 \mathrm{~m}$

Habitat:

Primary lowland forest, canopy 10-60 m high; associates diverse, include: Sterculia, Knema, Polyathia, Pometia.

Field Char:

Canopy tree $20 \mathrm{~m}$ tall, branching above and below; bole cylindrical, $30 \mathrm{~cm}$ diam., bark pale gray, rough; foliage dark green, new leaves glossy and reddish; flowers abundant, tepals 3, pale green, reflexed, receptacle swollen, bearing ca. 30 stamens, filaments white.

McDonald \& Ismail 3745

29 May 1993

\section{HERBARIUM BOGORIENSE HARVARD UNIVERSITY HIERBARIA}

\section{PLANTS OF INDONESIA}

Proteaceae

Cat. \# 3746

\section{Finschia chloroxantha Diels}

Det. By: McDonald 1993

Locality:

New Guinea; Sarmi,

Coastal plain and Gunung Top, W of Sungai Tor, E of Sungai Waske, vic. of Sewan village on Titve stream.

$2^{\circ} 4^{\prime}$ S $138^{\circ} 46^{\prime} \mathrm{E}$ Alt: $10-100 \mathrm{~m}$

Habitat:

Primary lowland forest, canopy 10-60 m high; associates diverse, include: Sterculia, Knema, Polyathia, Pometia.

Field Char:

Canopy tree $30 \mathrm{~m}$ tall, branching above, bole crooked, cylindrical, $35 \mathrm{~cm}$ diam.; bark tan-pale gray, rough; flowers abundant, peduncle and corolla bright orange, ovary green, style yellow.

McDonald \& Ismail 3746

29 May 1993

HERBARIUM BOGORIENSE HARVARD UNIVERSITY HERBARIA 


\section{PLANTS OF INDONESIA}

Clusiaceae

Cat. \# 3747

Garcinia sp.

Det. By: McDonald 1993

Locality:

New Guinea; Sarmi,

Coastal plain and Gunung Top, W of Sungai Tor, E of Sungai Waske, vic. of Sewan village on Titve stream.

$2^{\circ} 4^{\prime} \mathrm{S} 138^{\circ} 46^{\prime} \mathrm{E}$ Alt: $10-100 \mathrm{~m}$

Habitat:

Primary lowland forest, canopy 10-60 m high; associates diverse, include: Sterculia, Knema, Polyathia, Pometia.

Field Char:

Understory tree $7 \mathrm{~m}$ tall, branching above; bole straight, slender, cylindrical; bark dark gray, smooth; foliage glossy, new leaves light green, mature leaves dark green; flower buds bright pale green.

McDonald \& Ismail 3747

29 May 1993

\section{HERBARIUM BOGORIENSE HARVARD UNIVERSITY HERBARIA}

\section{PLANTS OF INDONESIA}

Euphorbiaceae

Cat. \# 3748

Phyllanthus longipes A. Shaw \& Webst

Det. By: McDonald 1993

Locality:

New Guinea; Sarmi,

Coastal plain and Gunung Top, W of Sungai Tor, E of Sungai Waske, vic. of Sewan village on Titve stream.

$2^{\circ} 4^{\prime} \mathrm{S} 138^{\circ} 46^{\prime} \mathrm{E}$ Alt: $10-100 \mathrm{~m}$

Habitat:

Primary lowland forest, canopy 10-60 m high; associates diverse, include: Sterculia, Knema, Polyathia, Pometia.

Field Char:

Midstory tree $9 \mathrm{~m}$ tall, branching above and below; bole crooked, $12 \mathrm{~cm}$ diam.; bark grayish, rough; immature fruits light green, glossy.

McDonald \& Ismail 3748

29 May 1993

HERBARIUM BOGORIENSE HARVARD UNIVERSITY HERBARIA 


\title{
PLANTS OF INDONESIA
}

Icacinaceae

Cat. \# 3749

Gomphandra sp.

Det. By: McDonald 1993

Locality:

New Guinea; Sarmi,

Coastal plain and Gunung Top, W of Sungai Tor, E of Sungai Waske, vic. of Sewan village on Titve

stream.

$2^{\circ} 4^{\prime} \mathrm{S} 138^{\circ} 46^{\prime}$ E Alt: $10-100 \mathrm{~m}$

Habitat:

Primary lowland forest, canopy 10-60 m high; associates diverse, include: Sterculia, Knema, Polyathia, Pometia.

Field Char:

Midstory tree $12 \mathrm{~m}$ tall, branching midway up; bole straight, cylindrical; bark tan-gray, smoothish; foliage dark green, glossy; flowers in bud, very pale green.

McDonald \& Ismail 3749

29 May 1993

\author{
HERBARIUM BOGORIENSE \\ HARVARD UNIVERSITY HERBARIA
}

\section{PLANTS OF INDONESIA}

Sapotaceae

Cat. \# 3750

\section{Sapotaceae}

Det. By: McDonald 1993

Locality:

New Guinea; Sarmi,

Coastal plain and Gunung Top, W of Sungai Tor, E of Sungai Waske, vic. of Sewan village on Titve stream.

$2^{\circ} 4^{\prime}$ 'S $138^{\circ} 46^{\prime}$ E Alt: $10-100 \mathrm{~m}$

Habitat:

Primary lowland forest, canopy 10-60 m high; associates diverse, include: Sterculia, Knema, Polyathia, Pometia.

Field Char:

Canopy tree $33 \mathrm{~m}$ tall, branching above; bole straight, cylindrical, $40 \mathrm{~cm}$ diam.; bark pale gray, smoothish, latex white; sepals 5 , very pale green; corolla suburceolate, segments 5 , ovary discoid; fruits immature, round, $6-8 \mathrm{~cm}$ diam., green.

McDonald \& Ismail 3750

29 May 1993 


\section{PLANTS OF INDONESIA}

Cat. \# 3751

Alstonia?

Det. By: McDonald 1993

Locality:

New Guinea; Sarmi,

Coastal plain and Gunung Top, W of Sungai Tor, E of Sungai Waske, vic. of Sewan village on Titve stream.

$2^{\circ} 4^{\prime} \mathrm{S} 138^{\circ} 46^{\prime} \mathrm{E}$ Alt: $10-100 \mathrm{~m}$

Habitat:

Primary lowland forest, canopy 10-60 m high; associates diverse, include: Sterculia, Knema, Polyathia, Pometia.

Field Char:

Midstory tree $16 \mathrm{~m}$ tall, branching above; bole somewhat contoured, straight, $30 \mathrm{~cm}$ diam.; bark pale tan-gray, smoothish with small protruding lenticels; corollas $5 \mathrm{~mm}$ long, subcampanulate, segments 5 , each notched.

McDonald \& Ismail 3751

29 May 1993

\section{HERBARIUM BOGORIENSE HARVARD UNIVERSITY HERBARIA}

\section{PLANTS OF INDONESIA}

Lauraceae

Cat. \# 3752

\section{Cryptocarya alleniana White}

Det. By: McDonald 1993

Locality:

New Guinea; Sarmi,

Coastal plain, 1-3 km N of Sewan on the Waske

River.

$2^{\circ} 4^{\prime}$ S $138^{\circ} 46^{\prime}$ E Alt: $10-20 \mathrm{~m}$

Habitat:

Primary lowland forest, canopy $25-45 \mathrm{~m}$ high, associates diverse, including Celtis, Sterculia, Pometia, Ficus, Oncospermum, and sundry

Rubiaceae.

Field Char:

Midstory tree $25 \mathrm{~m}$ tall, branching above, bole straight, cylindrical; buttressed at base; bark reddish, smooth; new growth stems somewhat angular; fruits very immature, green.

McDonald \& Ismail 3752 


\title{
PLANTS OF INDONESIA
}

Cat. \# 3753

Voacanga?

Det. By: McDonald 1993

Locality:

New Guinea; Sarmi,

Coastal plain, 1-3 km N of Sewan on the Waske

River.

$2^{\circ} 4^{\prime} \mathrm{S} 138^{\circ} 46^{\prime} \mathrm{E}$ Alt: $10-20 \mathrm{~m}$

Habitat:

Primary lowland forest, canopy $25-45 \mathrm{~m}$ high, associates diverse, including Celtis, Sterculia,

Pometia, Ficus, Oncospermum, and sundry

Rubiaceae.

Field Char:

Understory tree $8 \mathrm{~m}$ tall, branching midway up; bole straight, contoured, $12 \mathrm{~cm}$ diam.; bark brownish gray, rough, latex white; calyx green, corolla creampale green, $2.5 \mathrm{~cm}$ long; fruits light green, turning orange at maturity.

McDonald \& Ismail 3753

31 May 1993

\author{
HERBARIUM BOGORIENSE \\ HARVARD UNIVERSITY HERBARIA
}

\section{PLANTS OF INDONESIA}

Rubiaceae

Cat. \# 3754

Psychotria sp.

Det. By: McDonald 1993

Locality:

New Guinea; Sarmi,

Coastal plain, 1-3 km N of Sewan on the Waske

River.

$2^{\circ} 4^{\prime} \mathrm{S} 138^{\circ} 46^{\prime} \mathrm{E}$ Alt: $10-20 \mathrm{~m}$

Habitat:

Primary lowland forest, canopy 25-45 m high, associates diverse, including Celtis, Sterculia, Pometia, Ficus, Oncospermum, and sundry

Rubiaceae.

Field Char:

Shrubby, understory tree, $5 \mathrm{~m}$ tall, branching midway up, basal stem to $4 \mathrm{~cm}$ diam.; bark pale gray; fruits abundant, ellipsoid, white with green striations when young, maturing to glossy white and round. 


\section{PLANTS OF INDONESIA}

Apocynaceae

Cat. \# 3755

Apocynaceae

Det. By: McDonald 1993

Locality:

New Guinea; Sarmi,

Coastal plain, 1-3 km N of Sewan on the Waske

River.

$2^{\circ} 4^{\prime} \mathrm{S} 138^{\circ} 46^{\prime}$ E Alt: $10-20 \mathrm{~m}$

Habitat:

Primary lowland forest, canopy $25-45 \mathrm{~m}$ high, associates diverse, including Celtis, Sterculia,

Pometia, Ficus, Oncospermum, and sundry

Rubiaceae.

Field Char:

Shrubby, riparian, understory tree-shrub, branching above and below; trunk cylindrical, $15 \mathrm{~cm}$ diam.; bark pale tan, smooth; calyx urceolate, covering tube, corolla tube twisted, cream, segments twisted; immature fruits in pairs, green, rotund, ca. $5 \mathrm{~cm}$ diam.

McDonald \& Ismail 3755

31 May 1993

\section{HERBARIUM BOGORIENSE HARVARD UNIVERSITY HERBARIA}

\section{PLANTS OF INDONESIA}

Combretaceae

Cat. \# 3756

Terminalia cf. rubiginosa $K$. Sch.

Det. By: McDonald 1993

Locality:

New Guinea; Sarmi,

Coastal plain, 1-3 km N of Sewan on the Waske

River.

$2^{\circ} 4^{\prime} \mathrm{S} 138^{\circ} 46^{\prime} \mathrm{E}$ Alt: $10-20 \mathrm{~m}$

Habitat:

Primary lowland forest, canopy $25-45 \mathrm{~m}$ high, associates diverse, including Celtis, Sterculia, Pometia, Ficus, Oncospermum, and sundry Rubiaceae.

Field Char:

Midstory tree $18 \mathrm{~m}$ tall, branching above; bole straight, $20 \mathrm{~cm}$ diam.; new growth ruddy-pubescent; corolla white, ca. $5 \mathrm{~mm}$ diam., anthers yellow, style erect. 


\section{PLANTS OF INDONESIA}

Cat. \# 3757

\section{Polyalthia?}

Det. By: McDonald 1993

Locality:

New Guinea; Sarmi,

Coastal plain, 1-3 km N of Sewan on the Waske

River.

$2^{\circ} 4^{\prime}$ S $138^{\circ} 46^{\prime}$ E Alt: $10-20 \mathrm{~m}$

\section{Habitat:}

Primary lowland forest, canopy $25-45 \mathrm{~m}$ high, associates diverse, including Celtis, Sterculia,

Pometia, Ficus, Oncospermum, and sundry

Rubiaceae.

Field Char:

Understory tree to $5 \mathrm{~m}$ tall, branching above and below; bole stright, cylindrical, $10 \mathrm{~cm}$. diam.; bark gray-brown, smooth; lateral branches perpendicularacute to trunk; fruits orange, 1-3 per flower.

McDonald \& Ismail 3757

31 May 1993

\section{HERBARIUM BOGORIENSE HARVARD UNIVERSITY HERBARIA}

\section{PLANTS OF INDONESIA}

Moraceae

Cat. \# 3758

Antiaris toxicaria Lesch.

Det. By: McDonald 1993

Locality:

New Guinea; Sarmi,

Coastal plain, 1-3 $\mathrm{km} \mathrm{N}$ of Sewan on the Waske

River.

$2^{\circ} 4^{\prime}$ 'S $138^{\circ} 46^{\prime}$ E Alt: $10-20 \mathrm{~m}$

Habitat:

Primary lowland forest, canopy $25-45 \mathrm{~m}$ high, associates diverse, including Celtis, Sterculia, Pometia, Ficus, Oncospermum, and sundry Rubiaceae.

Field Char:

Midstory tree $11 \mathrm{~m}$ tall, branching above; bole cylindrical, crooked, $10 \mathrm{~cm}$ diam.; bark tan, fissured longitudinally; inflorescence abundant, white or cream-yellow, roundish, to $1 \mathrm{~cm}$ diam.

McDonald \& Ismail 3758

HERBARIUM BOGORIENSE HARVARD UNIVERSITY HERBARIA 


\section{PLANTS OF INDONESIA}

Meliaceae

Cat. \# 3759

Chisocheton cumingianus (DC.) Harms cumingianus

Det. By: McDonald 1993

Locality:

New Guinea; Sarmi,

Coastal plain, $1-3 \mathrm{~km} \mathrm{~N}$ of Sewan on the Waske

River.

$2^{\circ} 4^{\prime}$ 'S $138^{\circ} 46^{\prime}$ E Alt: $10-20 \mathrm{~m}$

Habitat:

Primary lowland forest, canopy $25-45 \mathrm{~m}$ high, associates diverse, including Celtis, Sterculia, Pometia, Ficus, Oncospermum, and sundry

Rubiaceae.

Field Char:

Canopy tree, $45 \mathrm{~m}$ tall, branching midway up; bole somewhat crooked, much contoured, buttressed at base, $60 \mathrm{~cm}$ diam.; bark tan-gray, smooth, sparingly flaky; flowers abundant, cauliflorous on lateral branches, in bud, corollas tubular, yellow-green.

McDonald \& Ismail 3759

31 May 1993

\section{HERBARIUM BOGORIENSE HARVARD UNIVERSITY HERBARIA}

\section{PLANTS OF INDONESIA}

Cat. \# 3760

Sloetia sp.

Det. By: McDonald 1993

Locality:

New Guinea; Sarmi,

Coastal plain, 1-3 km N of Sewan on the Waske

River.

$2^{\circ} 4^{\prime} \mathrm{S} 138^{\circ} 46^{\prime} \mathrm{E}$ Alt: $10-20 \mathrm{~m}$

Habitat:

Primary lowland forest, canopy 25-45 m high, associates diverse, including Celtis, Sterculia, Pometia, Ficus, Oncospermum, and sundry

Rubiaceae.

Field Char:

Short canopy tree $20 \mathrm{~m}$ tall, branching midway up; bole straight, cylindrical, $25 \mathrm{~cm}$ diam.; bark narrowly fissured, covered with orange and pale gray lichen; ovaries green, stigmas sub-plumose; fruits green and reddish (like immature apples). 


\section{PLANTS OF INDONESIA}

Rubiaceae

Cat. \# 3761

Timonius grandifolius Val.

Det. By: McDonald 1993

Locality:

New Guinea; Sarmi,

Coastal plain, 1-3 km N of Sewan on the Waske

River.

$2^{\circ} 4^{\prime} \mathrm{S} 138^{\circ} 46^{\prime} \mathrm{E}$ Alt: $10-20 \mathrm{~m}$

Habitat:

Primary lowland forest, canopy 25-45 m high, associates diverse, including Celtis, Sterculia, Pometia, Ficus, Oncospermum, and sundry

Rubiaceae.

Field Char:

Midstory tree $12 \mathrm{~m}$ tall, branching midway up; bole straight, cylindrical, $15 \mathrm{~cm}$ diam.; bark ruddy, somewhat flaky; foliage aggregated at stem apex; inflorescence light green-bracteate, calyx pale green, corolla cream.

McDonald \& Ismail 3761

31 May 1993

\section{HERBARIUM BOGORIENSE HARVARD UNIVERSITY HERBARIA}

\section{PLANTS OF INDONESIA}

Euphorbiaceae

Cat. \# 3762

Drypetes lasiogynoides Pax. \& Hof

Det. By: McDonald 1993

Locality:

New Guinea; Sarmi,

Coastal plain, 1-3 km $\mathrm{N}$ of Sewan on the Waske

River.

$2^{\circ} 4$ 'S $138^{\circ} 46^{\prime} \mathrm{E}$ Alt: $10-20 \mathrm{~m}$

Habitat:

Primary lowland forest, canopy $25-45 \mathrm{~m}$ high, associates diverse, including Celtis, Sterculia, Pometia, Ficus, Oncospermum, and sundry

Rubiaceae.

Field Char:

Midstory tree $15 \mathrm{~m}$ tall, branching midway up; bole straight, slightly contoured, $15 \mathrm{~cm}$. diam.; fruits fleshy, abundant, maturing from yellow to red.

McDonald \& Ismail 3762

1 June 1993

HERBARIUM BOGORIENSE HARVARD UNIVERSITY HERBARIA 


\section{PLANTS OF INDONESIA}

Arecaceae

Cat. \# 3763

Borassus Heineana Becc.

Det. By: McDonald 1993

Locality:

New Guinea; Sarmi,

Coastal plain, 1-3 km N of Sewan on the Waske

River.

$2^{\circ} 4^{\prime}$ S $138^{\circ} 46^{\prime}$ E Alt: $10-20 \mathrm{~m}$

Habitat:

Primary lowland forest, canopy $25-45 \mathrm{~m}$ high, associates diverse, including Celtis, Sterculia, Pometia, Ficus, Oncospermum, and sundry

Rubiaceae.

Field Char:

Midstory palm, $13 \mathrm{~m}$ tall; bole straight, $30 \mathrm{~cm}$ diam., internodes ca. $10 \mathrm{~cm}$ long; leaves $4-6 \mathrm{~cm}$ long, segments palmate, 50-60, lamina 1-2 m long, dark green, petioles $2-4 \mathrm{~m}$ long, $3-10 \mathrm{~cm}$ wide; fruits 5-7 per inflorescence, roundish, ca. $12 \mathrm{~cm}$ long, hard, woody, apex umbinate.

McDonald \& Ismail $3763 \quad 1$ June 1993

\section{HERBARIUM BOGORIENSE HARVARD UNIVERSITY HERBARIA}

\section{PLANTS OF INDONESIA}

Euphorbiaceae

Cat. \# 3764

Baccaurea sp.

Det. By: McDonald 1993

Locality:

New Guinea; Sarmi,

Coastal plain, 1-3 km N of Sewan on the Waske

River.

$2^{\circ} 4^{\prime}$ 'S $138^{\circ} 46^{\prime}$ E Alt: $10-20 \mathrm{~m}$

Habitat:

Primary lowland forest, canopy 25-45 m high, associates diverse, including Celtis, Sterculia, Pometia, Ficus, Oncospermum, and sundry Rubiaceae.

Field Char:

Canopy tree $30 \mathrm{~m}$ tall, branching above; bole striaght, cylindrical, $40 \mathrm{~cm}$ diam.; bark tan smoothish; fruits subrotund, orange at maturity.

McDonald \& Ismail 3764

1 June 1993

HERBARIUM BOGORIENSE HARVARD UNIVERSITY HERBARIA 


\section{PLANTS OF INDONESIA}

Cat. \# 3765

Sizygium cf. samarangense (Bl.) Merr.

Det. By: McDonald 1993

Locality:

New Guinea; Sarmi,

Coastal plain, 1-3 $\mathrm{km} \mathrm{N}$ of Sewan on the Waske

River.

$2^{\circ} 4^{\prime} \mathrm{S} 138^{\circ} 46^{\prime} \mathrm{E}$ Alt: $10-20 \mathrm{~m}$

Habitat:

Primary lowland forest, canopy $25-45 \mathrm{~m}$ high, associates diverse, including Celtis, Sterculia,

Pometia, Ficus, Oncospermum, and sundry

Rubiaceae.

Field Char:

Midstory tree, $17 \mathrm{~m}$ tall, branching above; bole straight, contoured, slightly buttressed at base; bark pale gray-tan, roughish; fruits abundant, turning scarlet at maturity, very sweet and sour.

McDonald \& Ismail 3765

1 June 1993

\section{HERBARIUM BOGORIENSE HARVARD UNIVERSITY HERBARIA}

\section{PLANTS OF INDONESIA}

Sapindaceae

Cat. \# 3766

Cupaniopsis sp.

Det. By: McDonald 1993

Locality:

New Guinea; Sarmi,

Coastal plain, 1-3 km N of Sewan on the Waske

River.

$2^{\circ} 4$ 'S $138^{\circ} 46^{\prime} \mathrm{E}$ Alt: $10-20 \mathrm{~m}$

Habitat:

Primary lowland forest, canopy $25-45 \mathrm{~m}$ high, associates diverse, including Celtis, Sterculia, Pometia, Ficus, Oncospermum, and sundry

Rubiaceae.

Field Char:

Canopy tree $40 \mathrm{~m}$ tall, branching midway up; bole straight, heavily buttressed at base, ca. $8 \mathrm{~m}$ wide at ground level, trunk $1.5 \mathrm{~m}$ diam.; bark reddish, somewhat patchy-flaky; flowers abundant, calyx light green, corolla urceolate, cream-pale green, gynoecium 3-carpellate, anthers greenish.

McDonald \& Ismail 3766

1 June 1993 


\section{PLANTS OF INDONESIA}

Lauraceae

Cat. \# 3767

Endiandra cf. brassii Allen

Det. By: McDonald 1993

Locality:

New Guinea; Sarmi,

Coastal plain, 1-3 km N of Sewan on the Waske

River.

$2^{\circ} 4^{\prime}$ 'S $138^{\circ} 46^{\prime}$ E Alt: $10-20 \mathrm{~m}$

Habitat:

Primary lowland forest, canopy $25-45 \mathrm{~m}$ high, associates diverse, including Celtis, Sterculia,

Pometia, Ficus, Oncospermum, and sundry

Rubiaceae.

Field Char:

Canopy tree $30 \mathrm{~m}$ tall, branching above; bole stright, cylindrical, $30 \mathrm{~cm}$ diam.; bark pale gray, smoothish, petals 6 , yellow-orange.

McDonald \& Ismail 3767

1 June 1993

\section{HERBARIUM BOGORIENSE \\ HARVARD UNIVERSITY HERBARIA}

\section{PLANTS OF INDONESIA}

Polygalaceae

Cat. \# 3768

Xanthophyllum papuanum Melch.

Det. By: McDonald 1993

Locality:

New Guinea; Sarmi,

Coastal plain, 1-3 km N of Sewan on the Waske

River.

$2^{\circ} 4^{\prime}$ 'S $138^{\circ} 46^{\prime}$ E Alt: $10-20 \mathrm{~m}$

Habitat:

Primary lowland forest, canopy $25-45 \mathrm{~m}$ high, associates diverse, including Celtis, Sterculia, Pometia, Ficus, Oncospermum, and sundry Rubiaceae.

Field Char:

Tall midstory tree $15 \mathrm{~m}$ tall, branching midway up; bole straight, cylindrical, slender, $15 \mathrm{~cm}$ diam.; bark pale gray, smoothish; flowers in bud, buds very pale green.

McDonald \& Ismail 3768

1 June 1993

HERBARIUM BOGORIENSE HARVARD UNIVERSITY HERBARIA 


\section{PLANTS OF INDONESIA}

Cat. \# 3769

Antiariopsis decipiens $\mathrm{K}$. Sch.

Det. By: McDonald 1993

Locality:

New Guinea; Sarmi,

Coastal plain, 1-3 km N of Sewan on the Waske

River.

$2^{\circ} 4^{\prime}$ 'S $138^{\circ} 46^{\prime}$ E Alt: $10-20 \mathrm{~m}$

Habitat:

Primary lowland forest, canopy $25-45 \mathrm{~m}$ high, associates diverse, including Celtis, Sterculia,

Pometia, Ficus, Oncospermum, and sundry

Rubiaceae.

Field Char:

Understory tree $5 \mathrm{~m}$ tall, branching above and below; bole straight, cylindrical, $8 \mathrm{~cm}$ diam.; bark pale gray, smoothish; inflorescence compact, bracteate head, yellowish with exserted styles at apex during anthesis, bright red and exposing white, gelatinous fruits during fruition.

McDonald \& Ismail 3769

1 June 1993

\section{HERBARIUM BOGORIENSE \\ HARVARD UNIVERSITY HERBARIA}

\section{PLANTS OF INDONESIA}

Hernandiaceae

Cat. \# 3770

Hernandia aff. ovigera $L$.

Det. By: McDonald 1993

Locality:

New Guinea; Sarmi,

Coastal plain, 1-3 $\mathrm{km} \mathrm{N}$ of Sewan on the Waske

River.

$2^{\circ} 4^{\prime}$ 'S $138^{\circ} 46^{\prime}$ E Alt: $10-20 \mathrm{~m}$

Habitat:

Primary lowland forest, canopy $25-45 \mathrm{~m}$ high, associates diverse, including Celtis, Sterculia,

Pometia, Ficus, Oncospermum, and sundry

Rubiaceae.

Field Char:

Canopy tree $40 \mathrm{~m}$ tall, branching midway up; bole straight, cylindrical, $1 \mathrm{~m}$ diam.; bark tan, narrowly fissured; foliage dark green, leaves glossy, calyx urceolate, pale green, petals white, stigma white, capitate, flowers protogynous, anthers yellow-orange.

McDonald \& Ismail 3770

1 June 1993

HERBARIUM BOGORIENSE

HARVARD UNIVERSITY HERBARIA 


\section{PLANTS OF INDONESIA}

Rubiaceae

Cat. \# 3771

Psychotria sp.

Det. By: McDonald 1993

Locality:

New Guinea; Sarmi,

Coastal plain, 1-3 km N of Sewan on the Waske

River.

$2^{\circ} 4^{\prime}$ 'S $138^{\circ} 46^{\prime}$ E Alt: $10-20 \mathrm{~m}$

Habitat:

Primary lowland forest, canopy $25-45 \mathrm{~m}$ high, associates diverse, including Celtis, Sterculia,

Pometia, Ficus, Oncospermum, and sundry

Rubiaceae.

Field Char:

Understory tree $8 \mathrm{~m}$ tall, branching midway up; bole straight, cylindrical, $15 \mathrm{~cm}$ diam.; bark tan-gray, narrowly fissured; leaves glossy; flowers ca. $1 \mathrm{~mm}$ long and wide, light yellow-green.

McDonald \& Ismail 3771

2 June 1993

\section{HERBARIUM BOGORIENSE HARVARD UNIVERSITY HERBARIA}

\section{PLANTS OF INDONESIA}

Sterculiaceae

Cat. \# 3772

Sterculia sp.

Det. By: McDonald 1993

Locality:

New Guinea; Sarmi,

Coastal plain, 1-3 $\mathrm{km} \mathrm{N}$ of Sewan on the Waske

River.

$2^{\circ} 4^{\prime}$ 'S $138^{\circ} 46^{\prime}$ 'E Alt: $10-20 \mathrm{~m}$

Habitat:

Primary lowland forest, canopy 25-45 m high, associates diverse, including Celtis, Sterculia, Pometia, Ficus, Oncospermum, and sundry Rubiaceae.

Field Char:

Canopy tree $40 \mathrm{~m}$ tall, branching above; bole straight, cylindrical, buttressed at base, trunk $80 \mathrm{~cm}$. diam.; bark tannish, smooth or narrowly grooved longitudinally; calyx orange green, reddish inside.

McDonald \& Ismail 3772

2 June 1993

HERBARIUM BOGORIENSE HARVARD UNIVERSITY HERBARIA 


\section{PLANTS OF INDONESIA}

Rubiaceae

Cat. \# 3773

Timonius sp.

Det. By: McDonald 1993

Locality:

New Guinea; Sarmi,

Coastal plain, $1-3 \mathrm{~km} \mathrm{~N}$ of Sewan on the Waske

River.

$2^{\circ} 4^{\prime} \mathrm{S} 138^{\circ} 46^{\prime} \mathrm{E}$ Alt: $10-20 \mathrm{~m}$

Habitat:

Primary lowland forest, canopy $25-45 \mathrm{~m}$ high, associates diverse, including Celtis, Sterculia, Pometia, Ficus, Oncospermum, and sundry

Rubiaceae.

Field Char:

Midstory tree $13 \mathrm{~m}$ tall; branching above; bole straight, cylindrical, $15 \mathrm{~cm}$ diam.; bark gray, breaking into irregular plates; corolla yellow, infundibular, coriaceous; fruits immature, rotund, green.

McDonald \& Ismail 3773

2 June 1993

\section{HERBARIUM BOGORIENSE HARVARD UNIVERSITY HERBARIA}

\section{PLANTS OF INDONESIA}

Tiliaceae

Cat. \# 3774

Microcos $c f$. pentandra Burrett

Det. By: McDonald 1993

Locality:

New Guinea; Sarmi,

Coastal plain, 1-3 km N of Sewan on the Waske

River.

$2^{\circ} 4^{\prime} \mathrm{S} 138^{\circ} 46^{\prime} \mathrm{E}$ Alt: $10-20 \mathrm{~m}$

Habitat:

Primary lowl and forest, canopy $25-45 \mathrm{~m}$ high, associates diverse, including Celtis, Sterculia, Pometia, Ficus, Oncospermum, and sundry Rubiaceae.

Field Char:

Canopy tree $25 \mathrm{~m}$ tall, branching above; bole straight, contoured, $35 \mathrm{~cm}$ diam., somewhat buttressed at base; bark gray-brown, smoothish; fruits abundant, rhomboid, exterior red, orange and fibrous within. 


\section{PLANTS OF INDONESIA}

Agavaceae

Cat. \# 3775

Dracaena angustifolia Roxb.

Det. By: McDonald 1993

Locality:

New Guinea; Sarmi,

Coastal plain, $1-3 \mathrm{~km} \mathrm{~N}$ of Sewan on the Waske

River.

$2^{\circ} 4^{\prime} \mathrm{S} 138^{\circ} 46^{\prime} \mathrm{E}$ Alt: $10-20 \mathrm{~m}$

Habitat:

Primary lowland forest, canopy $25-45 \mathrm{~m}$ high, associates diverse, including Celtis, Sterculia,

Pometia, Ficus, Oncospermum, and sundry

Rubiaceae.

Field Char:

Understory tree $8 \mathrm{~m}$ tall, branching above and below; basal stems 4-10 cm diam.; bark tannish-gray,

rough; lateral branches arching; fruits orange, rotund.

McDonald \& Ismail 3775

2 June 1993

\section{HERBARIUM BOGORIENSE \\ HARVARD UNIVERSITY HERBARIA}

\section{PLANTS OF INDONESIA}

Apocynaceae

Cat. \# 3776

Cerbera floribunda K. Sch.

Det. By: McDonald 1993

Locality:

New Guinea; Sarmi,

Coastal plain, 1-3 km N of Sewan on the Waske

River.

$2^{\circ} 4^{\prime}$ 'S $138^{\circ} 46^{\prime} \mathrm{E}$ Alt: $10-20 \mathrm{~m}$

Habitat:

Primary lowland forest, canopy $25-45 \mathrm{~m}$ high, associates diverse, including Celtis, Sterculia, Pometia, Ficus, Oncospermum, and sundry Rubiaceae.

Field Char:

Canopy tree $33 \mathrm{~m}$ tall, branching above; bole straight, cylindrical, $22 \mathrm{~cm}$ diam.; bark dark brown, smooth, but with distinctive, horizontal, protruding, ellipsoid lenticels to $8 \mathrm{~mm}$ long, latex white, abundant; calyx light green, lobes reflexed; corollas white and purple. 


\section{PLANTS OF INDONESIA}

Cat. \# 3777

Neiospermum citriodora

Det. By: McDonald 1993

Locality:

New Guinea; Sarmi,

Coastal plain, 1-3 km N of Sewan on the Waske

River.

$2^{\circ} 4^{\prime}$ S $138^{\circ} 46^{\prime}$ E Alt: $10-20 \mathrm{~m}$

Habitat:

Primary lowland forest, canopy $25-45 \mathrm{~m}$ high, associates diverse, including Celtis, Sterculia,

Pometia, Ficus, Oncospermum, and sundry

Rubiaceae.

Field Char:

Midstory tree $12 \mathrm{~m}$ tall, branching midway up, lateral branches perpendicular to trunk, whorled; bole straight, cylindrical, $8 \mathrm{~cm}$ diam.; bark dark brown, smooth; latex white, sparing; flower buds white, corollas ca. $7 \mathrm{~mm}$ long; fruits paired,

ellipsoid, horizontal.

McDonald \& Ismail 3777

2 June 1993

\section{HERBARIUM BOGORIENSE HARVARD UNIVERSITY HERBARIA}

\section{PLANTS OF INDONESIA}

Burseraceae

Cat. \# 3778

\section{Canarium sp.}

Det. By: McDonald 1993

Locality:

New Guinea; Sarmi,

Coastal plain, 1-3 km N of Sewan on the Waske

River.

$2^{\circ} 4^{\prime}$ 'S $138^{\circ} 46^{\prime}$ E Alt: $10-20 \mathrm{~m}$

Habitat:

Primary lowland forest, canopy $25-45 \mathrm{~m}$ high, associates diverse, including Celtis, Sterculia,

Pometia, Ficus, Oncospermum, and sundry

Rubiaceae.

Field Char:

Midstory tree $17 \mathrm{~m}$ tall, branching above; bole straight, cylindrical; bark tan, smoothish; flowers trimerous, calyx pale green, corolla whitish, 3-lobed, anthers yellow. 


\section{PLANTS OF INDONESIA}

Burseraceae

Cat. \# 3779

Canarium lamii Leenh.

Det. By: McDonald 1993

Locality:

New Guinea; Sarmi,

Coastal plain, 1-3 km N of Sewan on the Waske

River.

$2^{\circ} 4^{\prime} \mathrm{S} 138^{\circ} 46^{\prime} \mathrm{E}$ Alt: $10-20 \mathrm{~m}$

Habitat:

Primary lowland forest, canopy $25-45 \mathrm{~m}$ high, associates diverse, including Celtis, Sterculia, Pometia, Ficus, Oncospermum, and sundry

Rubiaceae.

Field Char:

Canopy tree $35 \mathrm{~m}$ tall, branching above; bole straight, cylindrical, $30 \mathrm{~cm}$ diam.; bark pale gray, smooth; fruits occasional, subpyriform, ca. $8 \mathrm{~cm}$ long, green glossy.

McDonald \& Ismail 3779

2 June 1993

\section{HERBARIUM BOGORIENSE HARVARD UNIVERSITY HERBARIA}

\section{PLANTS OF INDONESIA}

Annonaceae

Cat. \# 3780

\section{Polyalthia sp.}

Det. By: McDonald 1993

Locality:

New Guinea; Sarmi,

Coastal plain, 1-3 $\mathrm{km} \mathrm{N}$ of Sewan on the Waske

River.

$2^{\circ} 4^{\prime}$ 'S $138^{\circ} 46^{\prime}$ E Alt: $10-20 \mathrm{~m}$

Habitat:

Primary lowland forest, canopy $25-45 \mathrm{~m}$ high, associates diverse, including Celtis, Sterculia, Pometia, Ficus, Oncospermum, and sundry Rubiaceae.

Field Char:

Canopy tree $33 \mathrm{~m}$ tall, branching above, lateral branches perpendicular to trunk; bole straight, cylindrical, $30 \mathrm{~cm}$ diam.; bark rough, covered with multi-colored lichens; flowers cauliflorous on lateral branches, peduncles green-orangish, calyx green, perianth cream; imm. fruit grn 
Fabaceae

Cat. \# 3781

\section{Archidendron cf. parviflorum Pulle}

Det. By: McDonald 1993

Locality:

New Guinea; Sarmi,

Coastal plain, 1-3 $\mathrm{km} \mathrm{N}$ of Sewan on the Waske

River.

$2^{\circ} 4^{\prime} \mathrm{S} 138^{\circ} 46^{\prime} \mathrm{E}$ Alt: $10-20 \mathrm{~m}$

Habitat:

Primary lowland forest, canopy $25-45 \mathrm{~m}$ high, associates diverse, including Celtis, Sterculia, Pometia, Ficus, Oncospermum, and sundry Rubiaceae.

Field Char:

Midstory tree $12 \mathrm{~m}$ tall, branching above; bole somewhat crooked, cylindrical, $15 \mathrm{~cm}$ diam.; bark tan, smoothish; stems hollowed by ants; fruits cauliflorous, 1-3 per node, coiled, bright red, yellow inside, seeds black, exposed upon dehiscence.

McDonald \& Ismail 3781

3 June 1993

\section{HERBARIUM BOGORIENSE HARVARD UNIVERSITY HERBARIA}

\section{PLANTS OF INDONESIA}

Euphorbiaceae

Cat. \# 3782

\section{Croton pusilliflorus Croiz.}

Det. By: McDonald 1993

Locality:

New Guinea; Sarmi,

Coastal plain, 1-3 km N of Sewan on the Waske

River.

$2^{\circ} 4^{\prime}$ 'S $138^{\circ} 46^{\prime} \mathrm{E} \mathrm{Alt:} 10-20 \mathrm{~m}$

Habitat:

Primary lowland forest, canopy 25-45 m high, associates diverse, including Celtis, Sterculia, Pometia, Ficus, Oncospermum, and sundry Rubiaceae.

Field Char:

Understory tree $8 \mathrm{~m}$ tall, branching midway up; bole straight, cylindrical, $12 \mathrm{~cm}$ diam.; bark tan, smoothish; fruits immature, very pale green.

McDonald \& Ismail 3782

3 June 1993

\section{HERBARIUM BOGORIENSE HARVARD UNIVERSITY HERBARIA}




\section{PLANTS OF INDONESIA}

Melastomataceae

Cat. \# 3783

Astronia glomerata Mansf.

Det. By: McDonald 1993

Locality:

New Guinea; Sarmi,

Coastal plain, 1-3 $\mathrm{km} \mathrm{N}$ of Sewan on the Waske

River.

$2^{\circ} 4^{\prime}$ 'S $138^{\circ} 46^{\prime}$ E Alt: $10-20 \mathrm{~m}$

Habitat:

Primary lowland forest, canopy 25-45 m high, associates diverse, including Celtis, Sterculia, Pometia, Ficus, Oncospermum, and sundry Rubiaceae.

Field Char:

Tall midstory tree, $18 \mathrm{~m}$ tall, branching above; bole straight, much contoured, $25 \mathrm{~cm}$ diam.; wood orange, bark flaky; leaves green above, rusty below; peduncles and calyx rusty, corolla white, urceolate, anthers yellow.

McDonald \& Ismail 3783

3 June 1993

\section{HERBARIUM BOGORIENSE HARVARD UNIVERSITY HERBARIA}

\section{PLANTS OF INDONESIA}

Fabaceae

Cat. \# 3784

Albizia sp.

Det. By: McDonald 1993

Locality:

New Guinea; Sarmi,

Coastal plain, 1-3 km N of Sewan on the Waske

River.

$2^{\circ} 4^{\prime} \mathrm{S} 138^{\circ} 46^{\prime} \mathrm{E}$ Alt: $10-20 \mathrm{~m}$

Habitat:

Primary lowland forest, canopy $25-45 \mathrm{~m}$ high, associates diverse, including Celtis, Sterculia, Pometia, Ficus, Oncospermum, and sundry Rubiaceae.

Field Char:

Canopy tree $20 \mathrm{~m}$ tall, branching above; bole straight, cylindrical, $25 \mathrm{~cm}$ diam.; bark pale gray, smooth; leaflets pale green below, dark green above; flowers abundant, calyx green, stamen filaments whitish.

McDonald \& Ismail 3784

3 June 1993

HERBARIUM BOGORIENSE HARVARD UNIVERSITY HERBARIA 


\section{PLANTS OF INDONESIA}

Arecaceae

Cat. \# 3785

Ptychococcus archboldianus Burrett

Det. By: McDonald 1993

Locality:

New Guinea; Sarmi,

Coastal plain, 1-3 km N of Sewan on the Waske

River.

$2^{\circ} 4^{\prime} \mathrm{S} 138^{\circ} 46^{\prime} \mathrm{E}$ Alt: $10-20 \mathrm{~m}$

Habitat:

Primary lowland forest, canopy $25-45 \mathrm{~m}$ high, associates diverse, including Celtis, Sterculia,

Pometia, Ficus, Oncospermum, and sundry

Rubiaceae.

Field Char:

Occasional palm, $14 \mathrm{~m}$ tall; trunk straight, $15 \mathrm{~cm}$ diam.; leaves 4-5 $\mathrm{m}$ long, ca. $1.5 \mathrm{~m}$ wide;

inflorescence branching, ca, $1 \mathrm{~m}$ long and wide, ca.

15 ramifications; peduncles and flowers pale creamgreen; fruits immature, green.

McDonald \& Ismail 3785

3 June 1993

\section{HERBARIUM BOGORIENSE \\ HARVARD UNIVERSITY HERBARIA}

\section{PLANTS OF INDONESIA}

Meliaceae

Cat. \# 3786

Aglaia sp.

Det. By: McDonald 1993

Locality:

New Guinea; Sarmi,

Coastal plain, 1-3 km N of Sewan on the Waske

River.

$2^{\circ} 4^{\prime} \mathrm{S} 138^{\circ} 46^{\prime} \mathrm{E}$ Alt: $10-20 \mathrm{~m}$

Habitat:

Primary lowland forest, canopy 25-45 m high, associates diverse, including Celtis, Sterculia, Pometia, Ficus, Oncospermum, and sundry Rubiaceae.

Field Char:

Canopy tree $45 \mathrm{~m}$ tall, branching above; bole ca. 1 m diam., buttressed below; bark tan, smooth, somewhat round flaky; fruits $12-16 \mathrm{~cm}$ diameter, light brown, laticiferous, 3-lobed.

McDonald \& Ismail 3786

3 June 1993

HERBARIUM BOGORIENSE HARVARD UNIVERSITY HERBARIA 


\section{PLANTS OF INDONESIA}

Icacinaceae

Cat. \# 3787

Pseudobotrys cauliflorum (Pulle) Sleu

Det. By: McDonald 1993

Locality:

New Guinea; Sarmi,

Coastal plain, 1-3 km N of Sewan on the Waske

River.

$2^{\circ} 4^{\prime}$ S $138^{\circ} 46^{\prime}$ E Alt: $10-20 \mathrm{~m}$

Habitat:

Primary lowland forest, canopy $25-45 \mathrm{~m}$ high, associates diverse, including Celtis, Sterculia,

Pometia, Ficus, Oncospermum, and sundry

Rubiaceae.

Field Char:

Understory tree $8 \mathrm{~m}$ tall, branching midway up; bole straight, somewhat contoured, $10 \mathrm{~cm}$ diam.; fruits cauliflorous on trunk, sparing, rotund, metallic blue, ca. 4-5 cm diam.

McDonald \& Ismail 3787

3 June 1993

\section{HERBARIUM BOGORIENSE HARVARD UNIVERSITY HERBARIA}

\section{PLANTS OF INDONESIA}

Sapotaceae

Cat. \# 3788

Sapotaceae

Det. By: McDonald 1993

Locality:

New Guinea; Sarmi,

Coastal plain, 1-3 $\mathrm{km} \mathrm{N}$ of Sewan on the Waske

River.

$2^{\circ} 4^{\prime} \mathrm{S} 138^{\circ} 46^{\prime} \mathrm{E}$ Alt: $10-20 \mathrm{~m}$

Habitat:

Primary lowland forest, canopy 25-45 m high, associates diverse, including Celtis, Sterculia, Pometia, Ficus, Oncospermum, and sundry

Rubiaceae.

Field Char:

Midstory tree $20 \mathrm{~m}$ tall, branching midway up; bole somewhat crooked, cylindrical, $25 \mathrm{~cm}$ diam.; bark grayish, rough, somewhat flaky, latex white; flowers in bud. 


\section{PLANTS OF INDONESIA}

Rhamnaceae

Cat. \# 3789

Ziziphus djamuensis Laut.

Det. By: McDonald 1993

Locality:

New Guinea; Sarmi,

Coastal plain, 1-3 $\mathrm{km} \mathrm{N}$ of Sewan on the Waske

River.

$2^{\circ} 4^{\prime} \mathrm{S} 138^{\circ} 46^{\prime} \mathrm{E}$ Alt: $10-20 \mathrm{~m}$

Habitat:

Primary lowland forest, canopy $25-45 \mathrm{~m}$ high, associates diverse, including Celtis, Sterculia,

Pometia, Ficus, Oncospermum, and sundry

Rubiaceae.

Field Char:

Robust liana, climbing to $35 \mathrm{~m}, 8 \mathrm{~cm}$ diam. at base; bark tan, rough due to many lenticels; stems bearing stipulate thorns; calyx green, disk yellow, anthers pale yellow.

McDonald \& Ismail 3789

4 June 1993

HERBARIUM BOGORIENSE

HARVARD UNIVERSITY HERBARIA

\section{PLANTS OF INDONESIA}

Icacinaceae

Cat. \# 3790

Citronella suaveolens (Bl.) Howard

Det. By: McDonald 1993

Locality:

New Guinea; Sarmi,

Coastal plain, 1-3 km N of Sewan on the Waske

River.

$2^{\circ} 4$ 'S $138^{\circ} 46^{\prime}$ E Alt: $10-20 \mathrm{~m}$

Habitat:

Primary lowland forest, canopy $25-45 \mathrm{~m}$ high, associates diverse, including Celtis, Sterculia, Pometia, Ficus, Oncospermum, and sundry

Rubiaceae.

Field Char:

Midstory tree $12 \mathrm{~m}$ tall, branching above; bole straight, cylindrical, $15 \mathrm{~cm}$ diam.; bark tan, somewhat chartaceous-flaky; foliage light green; flowers pentamerous, corolla whitish, tubular in bud, stamens exserted between limb segments; fruits ellipsoid, black.

McDonald \& Ismail 3790

4 June 1993 


\title{
PLANTS OF INDONESIA
}

Icacinaceae

Cat. \# 3791

Gonocaryum litorale (Bl.) Sleum.

Det. By: McDonald 1993

Locality:

New Guinea; Sarmi,

Coastal plain, 1-3 $\mathrm{km} \mathrm{N}$ of Sewan on the Waske

River.

$2^{\circ} 4^{\prime} \mathrm{S} 138^{\circ} 46^{\prime}$ E Alt: $10-20 \mathrm{~m}$

Habitat:

Primary lowland forest, canopy $25-45 \mathrm{~m}$ high, associates diverse, including Celtis, Sterculia,

Pometia, Ficus, Oncospermum, and sundry

Rubiaceae.

Field Char:

Understory tree, $8 \mathrm{~m}$ tall, branching midway up; bole knobby cylindrical, $10 \mathrm{~cm}$ diam; bark tan, sparingly chartaceous-flaky; leaves dark green above, petioles pale orange; fruits pendulous from lateral branches, red.

McDonald \& Ismail 3791

4 June 1993

\section{HERBARIUM BOGORIENSE HARVARD UNIVERSITY HERBARIA}

\section{PLANTS OF INDONESIA}

Euphorbiaceae

Cat. \# 3792

Macaranga aff. tanarius (L.) Muell

Det. By: McDonald 1993

Locality:

New Guinea; Sarmi,

Coastal plain, 1-3 km N of Sewan on the Waske

River.

$2^{\circ} 4^{\prime} \mathrm{S} 138^{\circ} 46^{\prime} \mathrm{E}$ Alt: $10-20 \mathrm{~m}$

Habitat:

Primary lowland forest, canopy $25-45 \mathrm{~m}$ high, associates diverse, including Celtis, Sterculia,

Pometia, Ficus, Oncospermum, and sundry

Rubiaceae.

Field Char:

Midstory tree $14 \mathrm{~m}$ tall, branching midway up; bole crooked, cylindrical, $12 \mathrm{~cm}$ diam.; brak grayish, smooth; inflorescence yellowish.

McDonald \& Ismail 3792

4 June 1993

\author{
HERBARIUM BOGORIENSE \\ HARVARD UNIVERSITY HERBARIA
}




\section{PLANTS OF INDONESIA}

Clusiaceae

Cat. \# 3793

Garcinia dulcis Kurz

Det. By: McDonald 1993

Locality:

New Guinea; Sarmi,

Coastal plain, 1-3 km N of Sewan on the Waske

River.

$2^{\circ} 4^{\prime} \mathrm{S} 138^{\circ} 46^{\prime} \mathrm{E}$ Alt: $10-20 \mathrm{~m}$

Habitat:

Primary lowland forest, canopy $25-45 \mathrm{~m}$ high, associates diverse, including Celtis, Sterculia,

Pometia, Ficus, Oncospermum, and sundry

Rubiaceae.

Field Char:

Midstory tree $17 \mathrm{~m}$ tall, branching midway up, lateral branches perpendicular to trunk; bole cylindrical, $20 \mathrm{~cm}$ diam.; bark brown-gray, smoothish; foliage dark green; flowers in bud, buds pale green.

McDonald \& Ismail 3793

4 June 1993

\section{HERBARIUM BOGORIENSE HARVARD UNIVERSITY HERBARIA}

\section{PLANTS OF INDONESIA}

Myrtaceae

Cat. \# 3794

Eugenia sp.

Det. By: McDonald 1993

Locality:

New Guinea; Sarmi,

Coastal plain, 1-3 km N of Sewan on the Waske

River.

$2^{\circ} 4^{\prime} \mathrm{S} 138^{\circ} 46^{\prime} \mathrm{E}$ Alt: $10-20 \mathrm{~m}$

Habitat:

Primary lowland forest, canopy $25-45 \mathrm{~m}$ high, associates diverse, including Celtis, Sterculia, Pometia, Ficus, Oncospermum, and sundry

Rubiaceae.

Field Char:

Canopy tree $23 \mathrm{~m}$ tall, branching midway up; bole straight, twisted-contoured, buttressed at base; bark reddish brown, smooth; fruits (some) persistent, dry, woody, brown.

McDonald \& Ismail 3794

4 June 1993

HERBARIUM BOGORIENSE HARVARD UNIVERSITY HERBARIA 


\section{PLANTS OF INDONESIA}

Rubiaceae

Cat. \# 3795

Mastixiodendron pachyclados (Sch). Melch

Det. By: McDonald 1993

Locality:

New Guinea; Sarmi,

Coastal plain, 1-3 km N of Sewan on the Waske

River.

$2^{\circ} 4^{\prime} \mathrm{S} 138^{\circ} 46^{\prime} \mathrm{E}$ Alt: $10-20 \mathrm{~m}$

Habitat:

Primary lowland forest, canopy $25-45 \mathrm{~m}$ high, associates diverse, including Celtis, Sterculia, Pometia, Ficus, Oncospermum, and sundry Rubiaceae.

Field Char:

Canopy tree $35 \mathrm{~m}$ tall, branching above; bole crooked, knobby, contoured, $80 \mathrm{~cm}$ diam., much buttressed at base ( $6 \mathrm{~m}$ wide); leaves dark green above, light green below; fruits immature, green, apex with an apparent calyptrum (dehiscence unknown).

McDonald \& Ismail 3795

4 June 1993

\section{HERBARIUM BOGORIENSE HARVARD UNIVERSITY HERBARIA}

\section{PLAN'TS OF INDONESIA}

Meliaceae

Cat. \# 3796

Chisocheton ceramica Miq.

Det. By: McDonald 1993

Locality:

New Guinea; Sarmi,

Coastal plain, 1-3 km N of Sewan on the Waske

River.

$2^{\circ} 4^{\prime}$ S $138^{\circ} 46^{\prime} \mathrm{E}$ Alt: $10-20 \mathrm{~m}$

Habitat:

Primary lowland forest, canopy 25-45 m high, associates diverse, including Celtis, Sterculia, Pometia, Ficus, Oncospermum, and sundry Rubiaceae.

Field Char:

Canopy tree $20 \mathrm{~m}$ tall, branching above; bole straight, contoured, $40 \mathrm{~cm}$ diam., somewhat buttressed at base; fruits abundant, red, finely velvety-puberulent.

McDonald \& Ismail 3796

4 June 1993

HERBARIUM BOGORIENSE HARVARD UNIVERSITY HERBARIA 


\section{PLANTS OF INDONESIA}

Icacinaceae

Cat. \# 3797

\section{Stemonurus monticolus}

Det. By: McDonald 1993

Locality:

New Guinea; Sarmi,

Coastal plain, $1-3 \mathrm{~km} \mathrm{~N}$ of Sewan on the Waske

River.

$2^{\circ} 4^{\prime} \mathrm{S} 138^{\circ} 46^{\prime} \mathrm{E}$ Alt: $10-20 \mathrm{~m}$

Habitat:

Primary lowland forest, canopy 25-45 m high, associates diverse, including Celtis, Sterculia,

Pometia, Ficus, Oncospermum, and sundry

Rubiaceae.

Field Char:

Tall midstory tree, $18 \mathrm{~m}$ tall, branching midway up; bole straight, cylindrical, $22 \mathrm{~cm}$ diam.; bark brownish gray, smoothish with horizontal, girdling ridges; flowers in aestivation, buds sessile-umbellate, light yellow-green.

McDonald \& Ismail 3797

5 June 1993

\section{HERBARIUM BOGORIENSE HARVARD UNIVERSITY HERBARIA}

\section{PLANTS OF INDONESIA}

Cat. \# 3798

Sizygium cf. burepense Hart.\&Perr

Det. By: McDonald 1993

Locality:

New Guinea; Sarmi,

Coastal plain, 1-3 $\mathrm{km} \mathrm{N}$ of Sewan on the Waske

River.

$2^{\circ} 4^{\prime}$ S $138^{\circ} 46^{\prime}$ E Alt: $10-20 \mathrm{~m}$

Habitat:

Primary lowland forest, canopy $25-45 \mathrm{~m}$ high, associates diverse, including Celtis, Sterculia, Pometia, Ficus, Oncospermum, and sundry Rubiaceae.

Field Char:

Understory tree $7 \mathrm{~m}$ tall, branching above and below; bole straight, $8 \mathrm{~cm}$ diam.; bark orange-brown, chartaceous; flowers aestivating, cauliflorous on lateral branches, buds pink.

McDonald \& Ismail 3798

5 June 1993

HERBARIUM BOGORIENSE HARVARD UNIVERSITY HERBARIA 


\section{PLANTS OF INDONESIA}

Myrtaceae

Cat. \# 3799

Eugenia sp.

Det. By: McDonald 1993

Locality:

New Guinea; Sarmi,

Coastal plain, 1-3 km N of Sewan on the Waske

River.

$2^{\circ} 4^{\prime} \mathrm{S} 138^{\circ} 46^{\prime} \mathrm{E}$ Alt: $10-20 \mathrm{~m}$

Habitat:

Primary lowland forest, canopy 25-45 m high, associates diverse, including Celtis, Sterculia,

Pometia, Ficus, Oncospermum, and sundry

Rubiaceae.

Field Char:

Understory tree $9 \mathrm{~m}$ tall, branching midway up; bole straight, contoured-grooved, $12 \mathrm{~cm}$ diam.; lateral branches perpendicular to trunk, crooked; fruits in terminal heads, urceolate, pale green.

McDonald \& Ismail 3799

5 June 1993

\section{HERBARIUM BOGORIENSE HARVARD UNIVERSITY HERBARIA}

\section{PLANTS OF INDONESIA}

Myristicaceae

Cat. \# 3800

Myristica subulata Miq.

Det. By: McDonald 1993

Locality:

New Guinea; Sarmi,

Coastal plain, 1-3 km N of Sewan on the Waske

River.

$2^{\circ} 4^{\prime} \mathrm{S} 138^{\circ} 46^{\prime} \mathrm{E}$ Alt: $10-20 \mathrm{~m}$

Habitat:

Primary lowland forest, canopy 25-45 m high, associates diverse, including Celtis, Sterculia, Pometia, Ficus, Oncospermum, and sundry Rubiaceae.

Field Char:

Small understory tree, $4 \mathrm{~m}$ tall, branching above and below, lateral branches whorled; trunk straight, cylindrical, $3 \mathrm{~cm}$ diam.; lateral branches covered with muricate bodies that attract aggressive ants; flowers tubular, tube pale yellow and green, limb segments refelxed

McDonald \& Ismail 3800

5 June 1993

HERBARIUM BOGORIENSE HARVARD UNIVERSITY HERBARIA 


\section{PLANTS OF INDONESIA}

Annonaceae

Cat. \# 3801

Annonaceae

Det. By: McDonald 1993

Locality:

New Guinea; Sarmi,

Coastal plain, 1-3 km N of Sewan on the Waske

River.

$2^{\circ} 4^{\prime} \mathrm{S} 138^{\circ} 46^{\prime} \mathrm{E}$ Alt: $10-20 \mathrm{~m}$

Habitat:

Primary lowland forest, canopy 25-45 m high, associates diverse, including Celtis, Sterculia, Pometia, Ficus, Oncospermum, and sundry

Rubiaceae.

Field Char:

Midstory tree $12 \mathrm{~m}$ tall, branching midway up; bole straight, cylindrical, $20 \mathrm{~cm}$ diam.; bark tan-brown, smoothish; flowers cauliflorous on lateral branches, sweet-scented; tepals pale green, young anthers pinkish, calyx green.

McDonald \& Ismail 3801

5 June 1993

\section{HERBARIUM BOGORIENSE HARVARD UNIVERSITY HERBARIA}

\section{PLANTS OF INDONESIA}

Verbenaceae

Cat. \# 3802

Premna cf. integrifolia $L$.

Det. By: McDonald 1993

Locality:

New Guinea; Sarmi,

Coastal plain, 1-3 km N of Sewan on the Waske

River.

$2^{\circ} 4^{\prime} \mathrm{S} 138^{\circ} 46^{\prime} \mathrm{E}$ Alt: $10-20 \mathrm{~m}$

Habitat:

Primary lowland forest, canopy 25-45 m high, associates diverse, including Celtis, Sterculia, Pometia, Ficus, Oncospermum, and sundry Rubiaceae.

Field Char:

Midstory secondary tree, $7 \mathrm{~m}$ tall, branching midway up; bark pale gray, rough; fruits maturing from light green to black.

McDonald \& Ismail 3802

5 June 1993

HERBARIUM BOGORIENSE HARVARD UNIVERSITY HERBARIA 


\section{PLANTS OF INDONESIA}

Capparaceae

Cat. \# 3803

Capparis anceolaris DC.

Det. By: McDonald 1993

Locality:

New Guinea; Sarmi,

Coastal plain, 1-3 km N of Sewan on the Waske

River.

$2^{\circ} 4^{\prime}$ ' $138^{\circ} 46^{\prime}$ E Alt: $10-20 \mathrm{~m}$

Habitat:

Primary lowland forest, canopy $25-45 \mathrm{~m}$ high, associates diverse, including Celtis, Sterculia,

Pometia, Ficus, Oncospermum, and sundry

Rubiaceae.

Field Char:

Liana to $10 \mathrm{~m}$ high, lateral branches drooping, thorny; calyx light green corolla white, stamens numerous, ovary ca. $3 \mathrm{~mm}$ diam, suspended on a white gynophore ca. $3 \mathrm{~cm}$ long during anthesis.

McDonald \& Ismail 3803

7 June 1993

HERBARIUM BOGORIENSE HARVARD UNIVERSITY HERBARIA

\section{PLANTS OF INDONESIA}

Urticaceae

Cat. \# 3804

\section{Urticaceae}

Det. By: McDonald 1993

Locality:

New Guinea; Sarmi,

Coastal plain, 1-3 km N of Sewan on the Waske

River.

$2^{\circ} 4^{\prime}$ 'S $138^{\circ} 46^{\prime}$ E Alt: $10-20 \mathrm{~m}$

Habitat:

Primary lowland forest, canopy $25-45 \mathrm{~m}$ high, associates diverse, including Celtis, Sterculia, Pometia, Ficus, Oncospermum, and sundry Rubiaceae.

Field Char:

Riparian tree $7 \mathrm{~m}$ tall, branching above and below, overhanging river; bark pale gray; inflorescence yellow-greenish.

McDonald \& Ismail 3804

7 June 1993

HERBARIUM BOGORIENSE HARVARD UNIVERSITY HERBARIA 


\section{PLANTS OF INDONESIA}

Moraceae

Cat. \# 3805

Ficus pungens Reinw. \& Bl.

Det. By: McDonald 1993

Locality:

New Guinea; Sarmi,

Coastal plain, 1-3 km N of Sewan on the Waske

River.

$2^{\circ} 4^{\prime} \mathrm{S} 138^{\circ} 46^{\prime} \mathrm{E}$ Alt: $10-20 \mathrm{~m}$

Habitat:

Primary lowland forest, canopy 25-45 m high, associates diverse, including Celtis, Sterculia, Pometia, Ficus, Oncospermum, and sundry

Rubiaceae.

Field Char:

Riparian tree $10 \mathrm{~m}$ tall, overhanging river; bole cylindrical, $20 \mathrm{~cm}$ diam; bark pale gray, smooth; inflorescence cauliflorous on trunk, woody, branching, figs small, green, hard.

McDonald \& Ismail 3805

7 June 1993

HERBARIUM BOGORIENSE

HARVARD UNIVERSITY HERBARIA

\section{PLANTS OF INDONESIA}

Thymelaeaceae

Cat. \# 3806

Phaleria coccinea (Gaud.) Muell.

Det. By: McDonald 1993

Locality:

New Guinea; Sarmi,

Coastal plain, 1-3 km N of Sewan on the Waske

River.

$2^{\circ} 4^{\prime} \mathrm{S} 138^{\circ} 46^{\prime} \mathrm{E}$ Alt: $10-20 \mathrm{~m}$

Habitat:

Primary lowland forest, canopy $25-45 \mathrm{~m}$ high, associates diverse, including Celtis, Sterculia,

Pometia, Ficus, Oncospermum, and sundry

Rubiaceae.

Field Char:

Shrub $5 \mathrm{~m}$ tall, branching sparingly; stems dark brown, flowers white, stamens and style exserted ca $1 \mathrm{~cm}$ beyond corolla tube.

McDonald \& Ismail 3806

7 June 1993

HERBARIUM BOGORIENSE HARVARD UNIVERSITY HERBARIA 


\section{PLANTS OF INDONESIA}

Rubiaceae

Cat. \# 3807

Pavetta platyclada Laut. \& K.Sch

Det. By: McDonald 1993

Locality:

New Guinea; Sarmi,

Coastal plain, 1-3 km N of Sewan on the Waske

River.

$2^{\circ} 4^{\prime} \mathrm{S} 138^{\circ} 46^{\prime} \mathrm{E}$ Alt: $10-20 \mathrm{~m}$

Habitat:

Primary lowland forest, canopy $25-45 \mathrm{~m}$ high, associates diverse, including Celtis, Sterculia, Pometia, Ficus, Oncospermum, and sundry

Rubiaceae.

Field Char:

Understory shrub, $3 \mathrm{~m}$ tall, branching above; foliage glossy; calyx green, corolla white, style exserted.

McDonald \& Ismail 3807

7 June 1993

\section{HERBARIUM BOGORIENSE HARVARD UNIVERSITY HERBARIA}

\section{PLANTS OF INDONESIA}

Pittosporaceae

Cat. \# 3808

Pittosporum sinuatum $\boldsymbol{B l}$.

Det. By: McDonald 1993

Locality:

New Guinea; Sarmi,

Coastal plain, 1-3 km N of Sewan on the Waske

River.

$2^{\circ} 4^{\prime} \mathrm{S} 138^{\circ} 46^{\prime} \mathrm{E}$ Alt: $10-20 \mathrm{~m}$

Habitat:

Primary lowland forest, canopy $25-45 \mathrm{~m}$ high, associates diverse, including Celtis, Sterculia, Pometia, Ficus, Oncospermum, and sundry

Rubiaceae.

Field Char:

Occasional understory shrub to $2 \mathrm{~m}$ tall, branching sparingly, stems brittle; fruit orange, succulentcoriaceous, dehiscent, exposing red-arillate seeds.

McDonald \& Ismail 3808

7 June 1993

HERBARIUM BOGORIENSE HARVARD UNIVERSITY HERBARIA 


\section{PLANTS OF INDONESIA}

Piperaceae

Cat. \# 3809

Piper mestonii Bailey

Det. By: McDonald 1993

Locality:

New Guinea; Sarmi,

Coastal plain, 1-3 $\mathrm{km} \mathrm{N}$ of Sewan on the Waske

River.

$2^{\circ} 4^{\prime}$ 'S $138^{\circ} 46^{\prime}$ E Alt: $10-20 \mathrm{~m}$

Habitat:

Primary lowland forest, canopy 25-45 m high, associates diverse, including Celtis, Sterculia,

Pometia, Ficus, Oncospermum, and sundry

Rubiaceae.

Field Char:

Liana, stems to $20 \mathrm{~m}$ long, $3 \mathrm{~cm}$ diam, branching;

inflorescenes abundant, scarlet.

McDonald \& Ismail 3809

7 June 1993

HERBARIUM BOGORIENSE

HARVARD UNIVERSITY HERBARIA

\section{PLANTS OF INDONESIA}

Euphorbiaceae

Cat. \# 3810

Phyllanthus sp.

Det. By: McDonald 1993

Locality:

New Guinea; Sarmi,

Coastal plain, 1-3 km N of Sewan on the Waske

River.

$2^{\circ} 4^{\prime}$ 'S $138^{\circ} 46^{\prime}$ E Alt: $10-20 \mathrm{~m}$

Habitat:

Primary lowland forest, canopy $25-45 \mathrm{~m}$ high, associates diverse, including Celtis, Sterculia, Pometia, Ficus, Oncospermum, and sundry

Rubiaceae.

Field Char:

Understory shrub $1.5 \mathrm{~m}$ tall, much branched; leaves pale green below, green above, berries reddish-pink.

McDonald \& Ismail 3810

7 June 1993

HERBARIUM BOGORIENSE HARVARD UNIVERSITY HERBARIA 


\section{PLANTS OF INDONESIA}

Rubiaceae

Cat. \# 3811

Ixora sp.

Det. By: McDonald 1993

Locality:

New Guinea; Sarmi,

Coastal plain, 1-3 km N of Sewan on the Waske

River.

$2^{\circ} 4^{\prime} \mathrm{S} 138^{\circ} 46^{\prime} \mathrm{E}$ Alt: $10-20 \mathrm{~m}$

Habitat:

Primary lowland forest, canopy 25-45 m high, associates diverse, including Celtis, Sterculia, Pometia, Ficus, Oncospermum, and sundry

Rubiaceae.

Field Char:

Understory shrub $3 \mathrm{~m}$ tall, branching sparingly; new growth stems chlorophyllous; inflorescence pendant, flowers in bud, ovary green, corolla tube pink at base, whitish above, limb segments green; fruits immature, green.

McDonald \& Ismail 3811

7 June 1993

\section{HERBARIUM BOGORIENSE HARVARD UNIVERSITY HERBARIA}

\section{PLANTS OF INDONESIA}

Anacardiaceae

Cat. \# 3812

Semecarpus magnificus $K$. Sch.

Det. By: McDonald 1993

Locality:

New Guinea; Sarmi,

Coastal plain, 1-3 km N of Sewan on the Waske

River.

$2^{\circ} 4^{\prime} \mathrm{S} 138^{\circ} 46^{\prime} \mathrm{E}$ Alt: $10-20 \mathrm{~m}$

Habitat:

Primary lowland forest, canopy $25-45 \mathrm{~m}$ high, associates diverse, including Celtis, Sterculia, Pometia, Ficus, Oncospermum, and sundry

Rubiaceae.

Field Char:

Understory shrubby tree, only branching from base, the foliage congregated at terminus of slender trunks; boles to $3 \mathrm{~cm}$ diam; latex black; inflorescence cauliflorous on base of bole, branching, flowers white, disk pale yellow.

McDonald \& Ismail 3812 


\section{PLANTS OF INDONESIA}

Rubiaceae

Cat. \# 3813

Mussaenda sp.

Det. By: McDonald 1993

Locality:

New Guinea; Sarmi,

Coastal plain, 1-3 km N of Sewan on the Waske

River.

$2^{\circ} 4^{\prime} \mathrm{S} 138^{\circ} 46^{\prime} \mathrm{E}$ Alt: $10-20 \mathrm{~m}$

Habitat:

Primary lowland forest, canopy 25-45 m high, associates diverse, including Celtis, Sterculia,

Pometia, Ficus, Oncospermum, and sundry

Rubiaceae.

Field Char:

Small shrub to $1 \mathrm{~m}$ tall, branching sparingly; leaves green below, green above, bracts white, corolla yellow.

McDonald \& Ismail 3813

7 June 1993

HERBARIUM BOGORIENSE

HARVARD UNIVERSITY HERBARIA

\section{PLANTS OF INDONESIA}

Rubiaceae

Cat. \# 3814

Amaracarpus sp.

Det. By: McDonald 1993

Locality:

New Guinea; Sarmi,

Coastal plain, 1-3 km N of Sewan on the Waske

River.

$2^{\circ} 4^{\prime}$ 'S $138^{\circ} 46^{\prime} \mathrm{E}$ Alt: $10-20 \mathrm{~m}$

Habitat:

Primary lowland forest, canopy $25-45 \mathrm{~m}$ high, associates diverse, including Celtis, Sterculia, Pometia, Ficus, Oncospermum, and sundry

Rubiaceae.

Field Char:

Undertory shrub $4 \mathrm{~m}$ tall, branched above and below; corolla white, ca. $6 \mathrm{~mm}$ long, fruits reddish.

McDonald \& Ismail 3814

7 June 1993

HERBARIUM BOGORIENSE HARVARD UNIVERSITY HERBARIA 


\section{PLANTS OF INDONESIA}

Myrsinaceae

Cat. \# 3815

Ardisia aff. arcuata Kan. \& Hat.

Det. By: McDonald 1993

Locality:

New Guinea; Sarmi,

Coastal plain, 1-3 km N of Sewan on the Waske

River.

$2^{\circ} 4^{\prime} \mathrm{S} 138^{\circ} 46^{\prime}$ E Alt: $10-20 \mathrm{~m}$

Habitat:

Primary lowland forest, canopy $25-45 \mathrm{~m}$ high, associates diverse, including Celtis, Sterculia,

Pometia, Ficus, Oncospermum, and sundry

Rubiaceae.

Field Char:

Understory shrub $2 \mathrm{~m}$ tall, branching sparingly, fruit pinkish red.

McDonald \& Ismail 3815

7 June 1993

HERBARIUM BOGORIENSE

HARVARD UNIVERSITY HERBARIA

\section{PLANTS OF INDONESIA}

Poaceae

Cat. \# 3816

Leptaspis urceolata (Roxb.) Judz.

Det. By: McDonald 1993

Locality:

New Guinea; Sarmi,

Coastal plain, 1-3 km N of Sewan on the Waske

River.

$2^{\circ} 4^{\prime} \mathrm{S} 138^{\circ} 46^{\prime} \mathrm{E}$ Alt: $10-20 \mathrm{~m}$

Habitat:

Primary lowland forest, canopy 25-45 m high, associates diverse, including Celtis, Sterculia, Pometia, Ficus, Oncospermum, and sundry

Rubiaceae.

Field Char:

Occasional perennial, understory herb to $1 \mathrm{~m}$ tall, fruits sticky.

McDonald \& Ismail 3816

7 June 1993

HERBARIUM BOGORIENSE HARVARD UNIVERSITY HERBARIA 


\section{PLANTS OF INDONESIA}

Araceae

Cat. \# 3817

Araceae

Det. By: McDonald 1993

Locality:

New Guinea; Sarmi,

Coastal plain, 1-3 km N of Sewan on the Waske

River.

$2^{\circ} 4^{\prime} \mathrm{S} 138^{\circ} 46^{\prime} \mathrm{E}$ Alt: $10-20 \mathrm{~m}$

Habitat:

Primary lowland forest, canopy $25-45 \mathrm{~m}$ high, associates diverse, including Celtis, Sterculia, Pometia, Ficus, Oncospermum, and sundry

Rubiaceae.

Field Char:

Understory perennial herb in temporally inundated areas, ca. 10 leaves per root stalk, petioles pale yellow and dark green, lamina sagittate, spadix the same; spathe maroon ouside, striate-maroon inside, broadly lanceolate, inflorescence yellowish.

McDonald \& Ismail 3817

7 June 1993

\section{HERBARIUM BOGORIENSE HARVARD UNIVERSITY HERBARIA}

\section{PLANTS OF INDONESIA}

Orchidaceae

Cat. \# 3818

Orchidaceae

Det. By: McDonald 1993

Locality:

New Guinea; Sarmi,

Coastal plain, 1-3 km N of Sewan on the Waske

River.

$2^{\circ} 4^{\prime} \mathrm{S} 138^{\circ} 46^{\prime} \mathrm{E}$ Alt: $10-20 \mathrm{~m}$

Habitat:

Primary lowland forest, canopy $25-45 \mathrm{~m}$ high, associates diverse, including Celtis, Sterculia, Pometia, Ficus, Oncospermum, and sundry

Rubiaceae.

Field Char:

Rare understory, solitary herb $1 \mathrm{~m}$ tall, terrestrial, suspended on stilt roots; branching sympodial; flowers white throughout, throat yellow.

McDonald \& Ismail 3818

7 June 1993

HERBARIUM BOGORIENSE HARVARD UNIVERSITY HERBARIA 


\section{PLANTS OF INDONESIA}

\section{Curcuma longa $L$.}

Det. By: McDonald 1993

Locality:

New Guinea; Sarmi,

Coastal plain, 1-3 km N of Sewan on the Waske

River.

$2^{\circ} 4^{\prime} \mathrm{S} 138^{\circ} 46^{\prime} \mathrm{E}$ Alt: $10-20 \mathrm{~m}$

Habitat:

Primary lowland forest, canopy 25-45 m high, associates diverse, including Celtis, Sterculia,

Pometia, Ficus, Oncospermum, and sundry

Rubiaceae.

Field Char:

Uncommon, understory herb, $50 \mathrm{~cm}$ tall, leaves light green; inflorescence bracts pink lavender at apex, grading into light breen below; corolla yellow.

McDonald \& Ismail 3819

7 June 1993

\section{HERBARIUM BOGORIENSE \\ HARVARD UNIVERSITY HERBARIA}

\section{PLANTS OF INDONESIA}

Cat. \# 3820

Comminsia gigantea (Schef.)K. Sch.

Det. By: McDonald 1993

Locality:

New Guinea; Sarmi,

Coastal plain, 1-3 km N of Sewan on the Waske

River.

$2^{\circ} 4^{\prime} \mathrm{S} 138^{\circ} 46^{\prime} \mathrm{E}$ Alt: $10-20 \mathrm{~m}$

Habitat:

Primary lowland forest, canopy $25-45 \mathrm{~m}$ high, associates diverse, including Celtis, Sterculia, Pometia, Ficus, Oncospermum, and sundry

Rubiaceae.

\section{Field Char:}

Tall, understory, perennial herb in temporally inundated areas, 2-3 m tall; flowers white, corolla limb ca. $3 \mathrm{~cm}$ diam; fruits very pale greenish, tube ca. $3 \mathrm{~cm}$ long. 


\title{
PLANTS OF INDONESIA
}

Zingiberaceae

Cat. \# 3821

Alpinia sp.

Det. By: McDonald 1993

Locality:

New Guinea; Sarmi,

Coastal plain, 1-3 km N of Sewan on the Waske

River.

$2^{\circ} 4^{\prime} \mathrm{S} 138^{\circ} 46^{\prime} \mathrm{E}$ Alt: $10-20 \mathrm{~m}$

Habitat:

Primary lowland forest, canopy $25-45 \mathrm{~m}$ high, associates diverse, including Celtis, Sterculia,

Pometia, Ficus, Oncospermum, and sundry

Rubiaceae.

Field Char:

Common shrubby herb in temporally inundated areas, 2-3 m tall; infructescence terminal; fruits red-orange, seeds black and orange.

McDonald \& Ismail 3821

7 June 1993

\author{
HERBARIUM BOGORIENSE \\ HARVARD UNIVERSITY HERBARIA
}

\section{PLANTS OF INDONESIA}

Zingiberaceae

Cat. \# 3822

Riedelia aff. corallina Val.

Det. By: McDonald 1993

Locality:

New Guinea; Sarmi,

Coastal plain, 1-3 km N of Sewan on the Waske

River.

$2^{\circ} 4^{\prime} \mathrm{S} 138^{\circ} 46^{\prime} \mathrm{E}$ Alt: $10-20 \mathrm{~m}$

Habitat:

Primary lowland forest, canopy $25-45 \mathrm{~m}$ high, associates diverse, including Celtis, Sterculia,

Pometia, Ficus, Oncospermum, and sundry

Rubiaceae.

Field Char:

Colonial herb forming shrub 1-2 $\mathrm{m}$ tall and wide,

leaves light green, fruits orange, seeds black, aril red, infructescence basal.

McDonald \& Ismail 3822

7 June 1993

HERBARIUM BOGORIENSE HARVARD UNIVERSITY HERBARIA 


\section{PLANTS OF INDONESIA}

Cat. \# 3823

Phyrnium macrocephalum K. Sch.

Det. By: McDonald 1993

Locality:

New Guinea; Sarmi,

Coastal plain, 1-3 km N of Sewan on the Waske

River.

$2^{\circ} 4^{\prime}$ 'S $138^{\circ} 46^{\prime}$ E Alt: $10-20 \mathrm{~m}$

Habitat:

Primary lowland forest, canopy $25-45 \mathrm{~m}$ high, associates diverse, including Celtis, Sterculia,

Pometia, Ficus, Oncospermum, and sundry

Rubiaceae.

Field Char:

Understory herb 1-1.5 $\mathrm{m}$ tall, fruits pinkish-lavender.

McDonald \& Ismail 3823

7 June 1993

\section{HERBARIUM BOGORIENSE \\ HARVARD UNIVERSITY HERBARIA}

\section{PLANTS OF INDONESIA}

Melastomataceae

Cat. \# 3824

Medinilla angustibasis Ohwi

Det. By: McDonald 1993

Locality:

New Guinea; Sarmi,

Coastal plain, 1-3 km N of Sewan on the Waske

River.

$2^{\circ} 4^{\prime} \mathrm{S} 138^{\circ} 46^{\prime} \mathrm{E}$ Alt: $10-20 \mathrm{~m}$

Habitat:

Primary lowland forest, canopy $25-45 \mathrm{~m}$ high, associates diverse, including Celtis, Sterculia,

Pometia, Ficus, Oncospermum, and sundry

Rubiaceae.

Field Char:

Liana $5 \mathrm{~m}$ tall, branching sparingly, lateral branches pendant; new stem growth angular; inflorescence branches bright red, berries white.

McDonald \& Ismail 3824

7 June 1993

HERBARIUM BOGORIENSE HARVARD UNIVERSITY HERBARIA 


\section{PLANTS OF INDONESIA}

Moraceae

Cat. \# 3825

Ficus adensosperma Miq.

Det. By: McDonald 1993

Locality:

New Guinea; Sarmi,

Valley of Gunung Top, vic. Sewan Village, W of

Sunga Tor, E of Sungai Waske, along Titfe Stream.

$2^{\circ} 4$ 'S $138^{\circ} 46^{\prime}$ E Alt: $10-100 \mathrm{~m}$

Habitat:

Primary lowland forest, canopy 10-60 m high;

associates include Sterculia, Knema, Polyalthia,

Pometia, etc.

Field Char:

Riparian tree $12 \mathrm{~cm}$ tall, overhanging river, branching above; bole bent, ellipsoidal, $30 \mathrm{~cm}$ diam; bark pale tan with gray patches; figs immature, pale green, at terminus of branches.

McDonald \& Ismail 3825

8 June 1993

HERBARIUM BOGORIENSE

HARVARD UNIVERSITY HERBARIA

\section{PLANTS OF INDONESIA}

Loranthaceae

Cat. \# 3826

Decaisnina hollrungii

Det. By: McDonald 1993

Locality:

New Guinea; Sarmi,

Valley of Gunung Top, vic. Sewan Village, W of

Sunga Tor, E of Sungai Waske, along Titfe Stream.

$2^{\circ} 4^{\prime} \mathrm{S} 138^{\circ} 46^{\prime} \mathrm{E}$ Alt: $10-100 \mathrm{~m}$

Habitat:

Primary lowland forest, canopy 10-60 m high;

associates include Sterculia, Knema, Polyalthia,

Pometia, etc.

Field Char:

Parasite of riparian fig tree, epiphyte, in canopy, shrubby, much branched, $1 \mathrm{~m}$ tall, $2 \mathrm{~m}$ wide;

bearing red seeds covered with mucilage.

McDonald \& Ismail 3826

8 June 1993

HERBARIUM BOGORIENSE

HARVARD UNIVERSITY HERBARIA 


\section{PLANTS OF INDONESIA}

Loranthaceae

Cat. \# 3827

Dendrophthoe falcata (L. f.) Etting.

Det. By: McDonald 1993

Locality:

New Guinea; Sarmi,

Valley of Gunung Top, vic. Sewan Village, W of

Sunga Tor, E of Sungai Waske, along Titfe Stream.

$2^{\circ} 4$ 'S $138^{\circ} 46^{\prime} \mathrm{E}$ Alt: $10-100 \mathrm{~m}$

Habitat:

Primary lowland forest, canopy 10-60 m high;

associates include Sterculia, Knema, Polyalthia,

Pometia, etc.

Field Char:

Parasite on riparian fig tree, epiphyte, shrub in

canopy, ca. $1 \mathrm{~m}$ tall, branching at base; calyx

greenish, corolla tube dark red, green at summit, limb segments orange, filaments red, stigma reddish.

McDonald \& Ismail 3827

8 June 1993

\section{HERBARIUM BOGORIENSE \\ HARVARD UNIVERSITY HERBARIA}

\section{PLANTS OF INDONESIA}

Myrtaceae

Cat. \# 3828

Eugenia sp.

Det. By: McDonald 1993

Locality:

New Guinea; Sarmi,

Valley of Gunung Top, vic. Sewan Village, W of

Sunga Tor, E of Sungai Waske, along Titfe Stream.

$2^{\circ} 4^{\prime} \mathrm{S} 138^{\circ} 46^{\prime} \mathrm{E}$ Alt: $10-100 \mathrm{~m}$

Habitat:

Primary lowland forest, canopy 10-60 m high; associates include Sterculia, Knema, Polyalthia, Pometia, etc.

Field Char:

Riparian tree overhanging river, $7 \mathrm{~m}$ tall, branching above, flowers almost open, buds pale green.

McDonald \& Ismail 3828

8 June 1993

HERBARIUM BOGORIENSE HARVARD UNIVERSITY HERBARIA 


\section{PLANTS OF INDONESIA}

Cat. \# 3829

Asplenium sp.

Det. By: McDonald 1993

Locality:

New Guinea; Sarmi,

Valley of Gunung Top, vic. Sewan Village, W of

Sunga Tor, E of Sungai Waske, along Titfe Stream.

$2^{\circ} 4^{\prime} \mathrm{S} 138^{\circ} 46^{\prime}$ E Alt: $10-100 \mathrm{~m}$

\section{Habitat:}

Primary lowland forest, canopy 10-60 m high;

associates include Sterculia, Knema, Polyalthia,

Pometia, etc.

Field Char:

Epiphytic fern on riparian Eugenia tree, fronds pale green, sori turning white to rusty.

McDonald \& Ismail 3829

8 June 1993

\section{HERBARIUM BOGORIENSE HARVARD UNIVERSITY HERBARIA}

\section{PLANTS OF INDONESIA}

Euphorbiaceae

Cat. \# 3830

\section{Euphorbiaceae}

Det. By: McDonald 1993

Locality:

New Guinea; Sarmi,

Valley of Gunung Top, vic. Sewan Village, W of

Sunga Tor, E of Sungai Waske, along Titfe Stream.

$2^{\circ} 4^{\prime} \mathrm{S} 138^{\circ} 46^{\prime} \mathrm{E}$ Alt: $10-100 \mathrm{~m}$

Habitat:

Primary lowland forest, canopy 10-60 m high;

associates include Sterculia, Knema, Polyalthia, Pometia, etc.

Field Char:

Riparian shrub $2 \mathrm{~m}$ tall, branching above, leaves pale green below, dark green above, corolla cream, pistil yellow.

McDonald \& Ismail 3830

8 June 1993

HERBARIUM BOGORIENSE HARVARD UNIVERSITY HERBARIA 


\section{PLANTS OF INDONESIA}

Cat. \# 3831

Freycinitia marantifolia Hemsl.

Det. By: McDonald 1993

Locality:

New Guinea; Sarmi,

Valley of Gunung Top, vic. Sewan Village, W of

Sunga Tor, E of Sungai Waske, along Titfe Stream.

$2^{\circ} 4^{\prime} \mathrm{S} 138^{\circ} 46^{\prime} \mathrm{E}$ Alt: $10-100 \mathrm{~m}$

Habitat:

Primary lowland forest, canopy 10-60 m high; associates include Sterculia, Knema, Polyalthia, Pometia, etc.

Field Char:

Riparian shrub, $1.5 \mathrm{~m}$ tall, branching sparingly at base; leaves light green, fruits bright, dark orange, fleshy-succulent.

McDonald \& Ismail $3831 \quad$ 8 June 1993

HERBARIUM BOGORIENSE

HARVARD UNIVERSITY HERBARIA

\section{PLANTS OF INDONESIA}

Acanthaceae

Cat. \# 3832

Hygrophila sp.

Det. By: McDonald 1993

Locality:

New Guinea; Sarmi,

Valley of Gunung Top, vic. Sewan Village, W of

Sunga Tor, E of Sungai Waske, along Titfe Stream.

$2^{\circ} 4^{\prime} \mathrm{S} 138^{\circ} 46^{\prime} \mathrm{E}$ Alt: $10-100 \mathrm{~m}$

Habitat:

Primary lowland forest, canopy $10-60 \mathrm{~m}$ high;

associates include Sterculia, Knema, Polyalthia,

Pometia, etc.

Field Char:

Riparian herb in rocks, corollas white.

McDonald \& Ismail 3832

8 June 1993

HERBARIUM BOGORIENSE

HARVARD UNIVERSITY HERBARIA 


\section{PLANTS OF INDONESIA}

Begoniaceae

Cat. \# 3833

Begonia weigalii Hemsl.

Det. By: McDonald 1993

Locality:

New Guinea; Sarmi,

Valley of Gunung Top, vic. Sewan Village, W of

Sunga Tor, E of Sungai Waske, along Titfe Stream.

$2^{\circ} 4^{\prime} \mathrm{S} 138^{\circ} 46^{\prime} \mathrm{E}$ Alt: $10-100 \mathrm{~m}$

Habitat:

Primary lowland forest, canopy 10-60 m high;

associates include Sterculia, Knema, Polyalthia,

Pometia, etc.

Field Char:

Riparian herb overhanging river on cliffs, stems succulent, flowers white and pink; fruits pendent, chartaceous.

McDonald \& Ismail 3833

8 June 1993

HERBARIUM BOGORIENSE

HARVARD UNIVERSITY HERBARIA

\section{PLANTS OF INDONESIA}

Heliconiaceae

Cat. \# 3834

\section{Heliconia papuana Kress}

Det. By: McDonald 1993

Locality:

New Guinea; Sarmi,

Valley of Gunung Top, vic. Sewan Village, W of

Sunga Tor, E of Sungai Waske, along Titfe Stream.

$2^{\circ} 4^{\prime} \mathrm{S} 138^{\circ} 46^{\prime}$ E Alt: $10-100 \mathrm{~m}$

Habitat:

Primary lowland forest, canopy 10-60 m high; associates include Sterculia, Knema, Polyalthia, Pometia, etc.

Field Char:

Subarborescent herb $3 \mathrm{~m}$ tall, inflorescence erect, bracts pale green, fruits immature, yellow.

McDonald \& Ismail 3834

8 June 1993

HERBARIUM BOGORIENSE HARVARD UNIVERSITY HERBARIA 


\section{PLANTS OF INDONESIA}

Convolvulaceae

Cat. \# 3835

Ipomoea aquatica Forsk.

Det. By: McDonald 1993

Locality:

New Guinea; Sarmi,

$25 \mathrm{~km}$ W of Sarmi on inland coastal road to Sewan.

$1^{\circ} 58^{\prime} \mathrm{S} 138^{\circ} 46^{\prime} \mathrm{E}$ Alt: $5 \mathrm{~m}$

Habitat:

Aquatic, roadside, inundated ditches. Dominants include Ipomoea, Cyperus, Eichhornia, Nymphaea, etc.

Field Char:

Trailing waterside plant, stems submerged, flowers and leaves emergent, corolla whitish, purple inside tube.

McDonald \& Ismail 3835

12 June 1993

\section{HERBARIUM BOGORIENSE \\ HARVARD UNIVERSITY HERBARIA}

\section{PLANTS OF INDONESIA}

Pontederiaceae

Cat. \# 3836

Monochoria sp.

Det. By: McDonald 1993

Locality:

New Guinea; Sarmi,

$25 \mathrm{~km} \mathrm{~W}$ of Sarmi on inland coastal road to Sewan.

$1^{\circ} 58^{\prime} \mathrm{S} 138^{\circ} 46^{\prime} \mathrm{E}$ Alt: $5 \mathrm{~m}$

Habitat:

Aquatic, roadside, inundated ditches. Dominants include Ipomoea, Cyperus, Eichhornia, Nymphaea, etc.

Field Char:

Emergent herb, leaves growing in rosettes, propagating asexually by underwater stolons, flowers yellow.

McDonald \& Ismail 3836

12 June 1993

HERBARIUM BOGORIENSE HARVARD UNIVERSITY HERBARIA 


\section{PLANTS OF INDONESIA}

Cyperaceae

Cat. \# 3837

Cyperus compactus Retz.

Det. By: McDonald 1993

Locality:

New Guinea; Sarmi,

$25 \mathrm{~km} \mathrm{~W}$ of Sarmi on inland coastal road to Sewan. $1^{\circ} 58^{\prime} \mathrm{S} 138^{\circ} 46^{\prime} \mathrm{E}$ Alt: $5 \mathrm{~m}$

Habitat:

Aquatic, roadside, inundated ditches. Dominants

include Ipomoea, Cyperus, Eichhornia, Nymphaea, etc.

Field Char:

Emergent herb $1 \mathrm{~m}$ tall, leaves in a loose rosette.

McDonald \& Ismail 3837

12 June 1993

HERBARIUM BOGORIENSE

HARVARD UNIVERSITY HERBARIA

\section{PLANTS OF INDONESIA}

Pontederiaceae

Cat. \# 3838

Monochoria vaginalis (Burm.) Presl.

Det. By: McDonald 1993

Locality:

New Guinea; Sarmi,

$25 \mathrm{~km} \mathrm{~W}$ of Sarmi on inland coastal road to Sewan.

$1^{\circ} 58^{\prime} \mathrm{S} 138^{\circ} 46^{\prime} \mathrm{E}$ Alt: $5 \mathrm{~m}$

Habitat:

Aquatic, roadside, inundated ditches. Dominants

include Ipomoea, Cyperus, Eichhornia, Nymphaea, etc.

Field Char:

Emergent herb, $0.5 \mathrm{~m}$ tall, leaves in a loose rosette, corolla light blue.

McDonald \& Ismail 3838

12 June 1993

HERBARIUM BOGORIENSE HARVARD UNIVERSITY HERBARIA 


\section{PLANTS OF INDONESIA}

Cat. \# 3839

Bacopa?

Det. By: McDonald 1993

Locality:

New Guinea; Sarmi,

$25 \mathrm{~km}$ W of Sarmi on inland coastal road to Sewan. $1^{\circ} 58^{\prime} \mathrm{S} 138^{\circ} 46^{\prime} \mathrm{E}$ Alt: $5 \mathrm{~m}$

Habitat:

Aquatic, roadside, inundated ditches. Dominants

include Ipomoea, Cyperus, Eichhornia, Nymphaea, etc.

Field Char:

Submerged herb, colonial, stem tips emergent with flowers, corollas whitish or very pale blue.

McDonald \& Ismail 3839

12 June 1993

\section{HERBARIUM BOGORIENSE HARVARD UNIVERSITY HERBARIA}

\section{PLANTS OF INDONESIA}

Nymphaeaceae

Cat. \# 3840

Nymphaea nouchalii Burm. $f$.

Det. By: McDonald 1993

Locality:

New Guinea; Sarmi,

$25 \mathrm{~km} \mathrm{~W}$ of Sarmi on inland coastal road to Sewan. $1^{\circ} 58^{\prime} \mathrm{S} 138^{\circ} 46^{\prime} \mathrm{E}$ Alt: $5 \mathrm{~m}$

Habitat:

Aquatic, roadside, inundated ditches. Dominants include Ipomoea, Cyperus, Eichhornia, Nymphaea, etc.

Field Char:

Occasional floating herb, flowers emergent, leaves green above, red with black punctae below, calyx green with small dark purple striations and punctae; corolla white, anthers cream, gynoecium bright yellow.

McDonald \& Ismail 3840

12 June 1993

HERBARIUM BOGORIENSE HARVARD UNIVERSITY HERBARIA 


\section{PLANTS OF INDONESIA}

Nymphaeaceae

Cat. \# 3841

Nymphaea pubescens Willd.

Det. By: McDonald 1993

Locality:

New Guinea; Jayapura,

Western outskirts of Sentani City, around paddies near shore of lake.

Alt: $20 \mathrm{~m}$

Habitat:

Aquatic, artificial fish ponds.

Field Char:

Floating aquatic, flowers diurnal, pinkish red, stamens red.

McDonald \& Ismail 3841

15 June 1993

HERBARIUM BOGORIENSE

HARVARD UNIVERSITY HERBARIA 


\section{PLANTS OF INDONESIA}

Pittosporaceae

Cat. \# 3842

Pittosporum ferrugineum Ait.

Det. By: McDonald 1993

Locality:

Kabaena; Kabaena,

Gunung Sabampolulu, $1 \mathrm{~km} \mathrm{S-SE} \mathrm{of} \mathrm{Tangkeno}$

$5^{\circ} 16^{\prime} \mathrm{S} 121^{\circ} 56^{\prime} \mathrm{E}$ Alt: $700-900 \mathrm{~m}$

Habitat:

W slopes of range, mixed ecotone of grasslands and short forest; arborescent associates include Grevillea, Helicia, Gardenia, Vaccinium, Eugenia; soils serpentine.

Field Char:

Occasional shrubby tree $5 \mathrm{~m}$ tall, branching sparingly midway up; corolla tubular, corolla tube 6$7 \mathrm{~mm}$ long, pale cream; anthers yellow, reaching to throat; fruits abundant, orange, fleshy, splitting.

McDonald \& Ismail 3842

21 July 1993

\section{HERBARIUM BOGORIENSE \\ HARVARD UNIVERSITY HERBARIA}

\section{PLANTS OF INDONESIA}

Epacridaceae

Cat. \# 3843

Styphelia abnormis (Sond.) J.J.S.

Det. By: McDonald 1993

Locality:

Kabaena; Kabaena,

Gunung Sabampolulu, $1 \mathrm{~km}$ S-SE of Tangkeno

$5^{\circ} 16^{\prime} \mathrm{S} 121^{\circ} 56^{\prime} \mathrm{E}$ Alt: $700-900 \mathrm{~m}$

Habitat:

W slopes of range, mixed ecotone of grasslands and short forest; arborescent associates include Grevillea, Helicia, Gardenia, Vaccinium, Eugenia; soils serpentine.

Field Char:

Subdominant shrub 1-1.5 m tall and wide, branching profusely; foliage light green, corolla white, urceolate, limb segments 5 , margins minutely fringed.

McDonald \& Ismail 3843

21 July 1993

\section{HERBARIUM BOGORIENSE HARVARD UNIVERSITY HERBARIA}




\section{PLANTS OF INDONESIA}

Cat. \# 3844

Sizygium zeylanicum (L.) DC.

Det. By: McDonald 1993

Locality:

Kabaena; Kabaena,

Gunung Sabampolulu, $1 \mathrm{~km} \mathrm{S-SE}$ of Tangkeno

$5^{\circ} 16^{\prime} \mathrm{S} 121^{\circ} 56^{\prime} \mathrm{E}$ Alt: $700-900 \mathrm{~m}$

Habitat:

W slopes of range, mixed ecotone of grasslands and short forest; arborescent associates include Grevillea, Helicia, Gardenia, Vaccinium, Eugenia; soils serpentine.

Field Char:

Uncommon tree $6 \mathrm{~m}$ tall, branching at base and above; 4 trunks, contoured, $10-15 \mathrm{~cm}$ diam.; bark chartaceous, exfoliating, tan-orange; foliage dark green, coriaceous; flowers abundant, calyx tube green, spent stamens reddish.

McDonald \& Ismail 3844

21 July 1993

\section{HERBARIUM BOGORIENSE HARVARD UNIVERSITY HERBARIA}

\section{PLANTS OF INDONESIA}

Araliaceae

Cat. \# 3845

Arthrophyllum cf. pacificum Philip.

Det. By: McDonald 1993

Locality:

Kabaena; Kabaena,

Gunung Sabampolulu, $1 \mathrm{~km} \mathrm{S-SE} \mathrm{of} \mathrm{Tangkeno}$

$5^{\circ} 16^{\prime} \mathrm{S} 121^{\circ} 56^{\prime} \mathrm{E}$ Alt: $700-900 \mathrm{~m}$

Habitat:

W slopes of range, mixed ecotone of grasslands and short forest; arborescent associates include Grevillea, Helicia, Gardenia, Vaccinium, Eugenia; soils serpentine.

Field Char:

Occasional tree 5-8 $\mathrm{m}$ tall, branching sparingly midway up; foliage present only at summit, light green; flowers abundant, inflorescence and flowers light green, floral disk olive, anthers yellow.

McDonald \& Ismail 3845

21 July 1993

HERBARIUM BOGORIENSE HARVARD UNIVERSITY HERBARIA 


\section{PLANTS OF INDONESIA}

Ericaceae

Cat. \# 3846

Vaccinium kjellbergii J.J.S.

Det. By: McDonald 1993

Locality:

Kabaena; Kabaena,

Gunung Sabampolulu, $1 \mathrm{~km}$ S-SE of Tangkeno

$5^{\circ} 16^{\prime} \mathrm{S} 121^{\circ} 56^{\prime} \mathrm{E}$ Alt: $700-900 \mathrm{~m}$

Habitat:

W slopes of range, mixed ecotone of grasslands and short forest; arborescent associates include Grevillea, Helicia, Gardenia, Vaccinium, Eugenia; soils serpentine.

Field Char:

Common shrub 1-3 m tall, branching above and below, stems crooked; flowers pendent, corolla conical-urceolate, pale pink, occasionally dark pinkstriate; fruits immature, green.

McDonald \& Ismail 3846

21 July 1993

HERBARIUM BOGORIENSE HARVARD UNIVERSITY HERBARIA

\section{PLANTS OF INDONESIA}

Caprifoliaceae

Cat. \# 3847

Viburnum cf. sambucinum $B l$.

Det. By: McDonald 1993

Locality:

Kabaena; Kabaena,

Gunung Sabampolulu, $1 \mathrm{~km}$ S-SE of Tangkeno

$5^{\circ} 16^{\prime} \mathrm{S} 121^{\circ} 56^{\prime} \mathrm{E}$ Alt: $700-900 \mathrm{~m}$

Habitat:

W slopes of range, mixed ecotone of grasslands and short forest; arborescent associates include Grevillea, Helicia, Gardenia, Vaccinium, Eugenia; soils serpentine.

Field Char:

Common shrub 3-5 $\mathrm{m}$ tall, branching profusely above; trunk $10 \mathrm{~cm}$ diam.; bark gray, smoothish; foliage light green, coriaceous; corollas white, stamens exserted; fruits green, turning red, flattenedellipsoid.

McDonald \& Ismail 3847

21 July 1993

HERBARIUM BOGORIENSE HARVARD UNIVERSITY HERBARIA 


\section{PLANTS OF INDONESIA}

Anacardiaceae

Cat. \# 3848

Buchanania arborescens (Bl.) Bl.

Det. By: McDonald 1993

Locality:

Kabaena; Kabaena,

Gunung Sabampolulu, $1 \mathrm{~km} \mathrm{S-SE} \mathrm{of} \mathrm{Tangkeno}$

$5^{\circ} 16^{\prime} \mathrm{S} 121^{\circ} 56^{\prime} \mathrm{E}$ Alt: $700-900 \mathrm{~m}$

Habitat:

W slopes of range, mixed ecotone of grasslands and short forest; arborescent associates include Grevillea, Helicia, Gardenia, Vaccinium, Eugenia; soils serpentine.

Field Char:

Occasional tree $11 \mathrm{~m}$ tall, branching above; bole straight, cylindrical, $20 \mathrm{~cm}$ diam.; bark brown, rough; inner bark reddish; flowers and inflorescence white, anthers yellow; fruits abundant, red above, green below.

McDonald \& Ismail 3848

21 July 1993

\section{HERBARIUM BOGORIENSE HARVARD UNIVERSITY HERBARIA}

\section{PLANTS OF INDONESIA}

Rhamnaceae

Cat. \# 3849

Alphitonia incana (Roxb.) Tiejs. \& Binn. ex Kurz

Det. By: McDonald 1993

Locality:

Kabaena; Kabaena,

Gunung Sabampolulu, $1 \mathrm{~km} \mathrm{S-SE} \mathrm{of} \mathrm{Tangkeno}$

$5^{\circ} 16^{\prime} \mathrm{S} 121^{\circ} 56^{\prime} \mathrm{E}$ Alt: $700-900 \mathrm{~m}$

Habitat:

W slopes of range, mixed ecotone of grasslands and short forest; arborescent associates include Grevillea, Helicia, Gardenia, Vaccinium, Eugenia; soils serpentine.

Field Char:

Uncommon canopy tree $15 \mathrm{~m}$ tall, branching above and below; trunk cylindrical, $30 \mathrm{~cm}$ diam.; bark tan, narrowly fissured; leaves shiny-silver below, green above; fruits abundant, black, seed orange; wood aromatic-spearmintish.

McDonald \& Ismail 3849

21 July 1993

HERBARIUM BOGORIENSE HARVARD UNIVERSITY HERBARIA 


\section{PLANTS OF INDONESIA}

Sterculiaceae

Cat. \# 3850

Sterculia cf. insularis $\boldsymbol{R}$. Br.

Det. By: McDonald 1993

Locality:

Kabaena; Kabaena,

Gunung Sabampolulu, $1 \mathrm{~km}$ S-SE of Tangkeno

$5^{\circ} 16^{\prime} \mathrm{S} 121^{\circ} 56^{\prime} \mathrm{E}$ Alt: $700-900 \mathrm{~m}$

Habitat:

W slopes of range, mixed ecotone of grasslands and short forest; arborescent associates include Grevillea, Helicia, Gardenia, Vaccinium, Eugenia; soils serpentine.

Field Char:

Occasional canopy tree $11 \mathrm{~m}$ tall, branching midway up; bole cylindrical, $20 \mathrm{~cm}$ diam.; bark tan-gray, rough, stems covered with ants; leaves coriaceous; inflorescence branches reddish, calyx tube greenish, red at base, limbs reddish-green, pubescent.

McDonald \& Ismail 3850

22 July 1993

\section{HERBARIUM BOGORIENSE HARVARD UNIVERSITY HERBARIA}

\section{PLANTS OF INDONESIA}

Burseraceae

Cat. \# 3851

\section{Canarium aspermum Benth.}

Det. By: McDonald 1993

Locality:

Kabaena; Kabaena,

Gunung Sabampolulu, $1 \mathrm{~km}$ S-SE of Tangkeno

$5^{\circ} 16^{\prime} \mathrm{S} 121^{\circ} 56^{\prime} \mathrm{E}$ Alt: $700-900 \mathrm{~m}$

Habitat:

W slopes of range, mixed ecotone of grasslands and short forest; arborescent associates include Grevillea, Helicia, Gardenia, Vaccinium, Eugenia; soils serpentine.

Field Char:

Common canopy tree $15 \mathrm{~m}$ tall, branching above; bole straight, cylindrical, $30 \mathrm{~cm}$ diam.; bark whitish, flaky, pinkish underneath; fruits abundant, immature green.

\section{HERBARIUM BOGORIENSE HARVARD UNIVERSITY HERBARIA}




\section{PLANTS OF INDONESIA}

Oxalidaceae

Cat. \# 3852

Sarcotheca celebica Veldkamp

Det. By: McDonald 1993

Locality:

Kabaena; Kabaena,

Gunung Sabampolulu, $1 \mathrm{~km}$ S-SE of Tangkeno

$5^{\circ} 16^{\prime} \mathrm{S} 121^{\circ} 56^{\prime} \mathrm{E}$ Alt: $700-900 \mathrm{~m}$

Habitat:

W slopes of range, mixed ecotone of grasslands and short forest; arborescent associates include Grevillea, Helicia, Gardenia, Vaccinium, Eugenia; soils serpentine.

Field Char:

Occasional forest-margin tree $8 \mathrm{~m}$ tall, branching midway up; bole straight, cylindrical, $12 \mathrm{~cm}$ diam.; bark tan, rough; leaves coriaceous, calyx green, petals maroon; fruit maroon, fleshy, segments 5 .

McDonald \& Ismail 3852

22 July 1993

HERBARIUM BOGORIENSE

HARVARD UNIVERSITY HERBARIA

\section{PLANTS OF INDONESIA}

Rosaceae

Cat. \# 3853

Prunus cf. grisea (Muell.)Kalk.

Det. By: McDonald 1993

Locality:

Kabaena; Kabaena,

Gunung Sabampolulu, $1 \mathrm{~km} \mathrm{S-SE} \mathrm{of} \mathrm{Tangkeno}$

$5^{\circ} 16^{\prime} \mathrm{S} 121^{\circ} 56^{\prime} \mathrm{E}$ Alt: $700-900 \mathrm{~m}$

Habitat:

W slopes of range, mixed ecotone of grasslands and short forest; arborescent associates include Grevillea, Helicia, Gardenia, Vaccinium, Eugenia; soils serpentine.

Field Char:

Occasional tree $12 \mathrm{~m}$ tall, branching midway up; bole cylindrical, $25 \mathrm{~cm}$ diam.; leaves coriaceous, green above, pale green below; fruits immature, light green.

McDonald \& Ismail 3853

22 July 1993

HERBARIUM BOGORIENSE HARVARD UNIVERSITY HERBARIA 


\section{PLANTS OF INDONESIA}

Myrtaceae

Xanthostemon confertiflorum Merr.

Det. By: McDonald 1993

Locality:

Kabaena; Kabaena,

Gunung Sabampolulu, $1 \mathrm{~km} \mathrm{S-SE}$ of Tangkeno

$5^{\circ} 16^{\prime} \mathrm{S} 121^{\circ} 56^{\prime} \mathrm{E}$ Alt: $700-900 \mathrm{~m}$

Habitat:

W slopes of range, mixed ecotone of grasslands and short forest; arborescent associates include Grevillea, Helicia, Gardenia, Vaccinium, Eugenia; soils serpentine.

Field Char:

Common forest-margin tree $5 \mathrm{~m}$ tall, branching at base and above; trunks 5-20 cm diam.; bark reddishbrown, flaky; flowers abundant, calyx green, petals red, stamens red, style red, ovary green.

McDonald \& Ismail 3854

22 July 1993

HERBARIUM BOGORIENSE HARVARD UNIVERSITY HERBARIA

\section{PLANTS OF INDONESIA}

Proteaceae

Cat. \# 3855

Grevillea elbertii Sleum.

Det. By: McDonald 1993

Locality:

Kabaena; Kabaena,

Gunung Sabampolulu, $1 \mathrm{~km} \mathrm{S-SE} \mathrm{of} \mathrm{Tangkeno}$

$5^{\circ} 16^{\prime} \mathrm{S} 121^{\circ} 56^{\prime} \mathrm{E}$ Alt: $700-900 \mathrm{~m}$

Habitat:

W slopes of range, mixed ecotone of grasslands and short forest; arborescent associates include Grevillea, Helicia, Gardenia, Vaccinium, Eugenia; soils serpentine.

Field Char:

Common forest-margin tree $8 \mathrm{~m}$ tall, branching midway up; bole $10 \mathrm{~cm}$ diam.; bark gray, narrowly and vertically grooved; leaves coppery-silver below, green above; flowers pale cream.

McDonald \& Ismail 3855

22 July 1993

\section{HERBARIUM BOGORIENSE HARVARD UNIVERSITY HERBARIA}




\section{PLANTS OF INDONESIA}

Casuarinaceae

Cat. \# 3856

\section{Gymnostoma sumatrana}

Det. By: McDonald 1993

Locality:

Kabaena; Kabaena,

Gunung Sabampolulu, $1 \mathrm{~km}$ S-SE of Tangkeno

$5^{\circ} 16^{\prime} \mathrm{S} 121^{\circ} 56^{\prime} \mathrm{E}$ Alt: $700-900 \mathrm{~m}$

Habitat:

W slopes of range, mixed ecotone of grasslands and short forest; arborescent associates include Grevillea, Helicia, Gardenia, Vaccinium, Eugenia; soils serpentine.

Field Char:

Common canopy tree to $35 \mathrm{~m}$ tall; bole straight, cylindrical, $70 \mathrm{~cm}$ diam.; lateral branches deciduous until summit; foliage dark green, dense.

McDonald \& Ismail 3856

22 July 1993

HERBARIUM BOGORIENSE HARVARD UNIVERSITY HERBARIA

\section{PLANTS OF INDONESIA}

Apocynaceae

Cat. \# 3857

Tabernaemontana sp.

Det. By: McDonald 1993

Locality:

Kabaena; Kabaena,

Gunung Sabampolulu, $1 \mathrm{~km}$ S-SE of Tangkeno $5^{\circ} 16^{\prime} \mathrm{S} 121^{\circ} 56^{\prime} \mathrm{E}$ Alt: $700-900 \mathrm{~m}$

Habitat:

W slopes of range, mixed ecotone of grasslands and short forest; arborescent associates include Grevillea, Helicia, Gardenia, Vaccinium, Eugenia; soils serpentine.

Field Char:

Occasional tree $15 \mathrm{~m}$ tall, branching midway up; bole straight, cylindrical, $50 \mathrm{~cm}$ diam.; bark pale gray, narrowly and vertically-fissured; lamina green with yellow venation above, latex white;

inflorescence branches reddish, calyx pale green, flowers white. 


\section{PLANTS OF INDONESIA}

Araliaceae

Cat. \# 3858

Polyscias nodosa (Bl.) Seem.

Det. By: McDonald 1993

Locality:

Kabaena; Kabaena,

Gunung Sabampolulu, $1 \mathrm{~km}$ S-SE of Tangkeno

$5^{\circ} 16^{\prime} \mathrm{S} 121^{\circ} 56^{\prime} \mathrm{E}$ Alt: $700-900 \mathrm{~m}$

Habitat:

W slopes of range, mixed ecotone of grasslands and short forest; arborescent associates include Grevillea, Helicia, Gardenia, Vaccinium, Eugenia; soils serpentine.

Field Char:

Occasional canopy tree $14 \mathrm{~m}$ tall, branching sparingly near summit, lateral branches whorled; bole straight, cylindrical; bark grayish, smooth above, vertically fissured below; foliage only at apex; inflorescence and flower buds green, terminal, ca. $50 \mathrm{~cm}$ long, $30 \mathrm{~cm}$ wide.

McDonald \& Ismail 3858

22 July 1993

\section{HERBARIUM BOGORIENSE HARVARD UNIVERSITY HERBARIA}

\section{PLANTS OF INDONESIA}

Myrtaceae

Cat. \# 3859

\section{Eugenia sp.}

Det. By: McDonald 1993

Locality:

Kabaena; Kabaena,

Gunung Sabampolulu, $1 \mathrm{~km}$ S-SE of Tangkeno

$5^{\circ} 16^{\prime} \mathrm{S} 121^{\circ} 56^{\prime} \mathrm{E}$ Alt: $700-900 \mathrm{~m}$

Habitat:

W slopes of range, mixed ecotone of grasslands and short forest; arborescent associates include Grevillea, Helicia, Gardenia, Vaccinium, Eugenia; soils serpentine.

Field Char:

Canopy tree $15 \mathrm{~m}$ tall, branching thrice from base and above; trunks cylindrical, $15-22 \mathrm{~cm}$ diam.; bark tannish-gray, very narrowly fissured; leaves coriaceous, green above, light green below; calyx light green, petals white, stamens white.

McDonald \& Ismail 3859 


\section{PLANTS OF INDONESIA}

Tiliaceae

Cat. \# 3860

Trichospermum kjellbergii Burrett

Det. By: McDonald 1993

Locality:

Kabaena; Kabaena,

Gunung Sabampolulu, $1 \mathrm{~km}$ S-SE of Tangkeno

$5^{\circ} 16^{\prime} \mathrm{S} 121^{\circ} 56^{\prime} \mathrm{E}$ Alt: $700-900 \mathrm{~m}$

Habitat:

W slopes of range, mixed ecotone of grasslands and short forest; arborescent associates include Grevillea, Helicia, Gardenia, Vaccinium, Eugenia; soils serpentine.

Field Char:

Occasional forest-margin tree $13 \mathrm{~m}$ tall, branching midway up; bole cylindrical, $20 \mathrm{~cm}$ diam.; bark pale gray, rough; leaves coriaceous, green above and below; flower buds brown-green, pubescent; fruits dry, covered with copper-urticating hairs; seeds black.

McDonald \& Ismail 3860

23 July 1993

\section{HERBARIUM BOGORIENSE HARVARD UNIVERSITY HERBARIA}

\section{PLANTS OF INDONESIA}

Aquifoliaceae

Cat. \# 3861

Ilex sp.

Det. By: McDonald 1993

Locality:

Kabaena; Kabaena,

Gunung Sabampolulu, $1 \mathrm{~km} \mathrm{S-SE} \mathrm{of} \mathrm{Tangkeno}$

$5^{\circ} 16^{\prime} \mathrm{S} 121^{\circ} 56^{\prime} \mathrm{E}$ Alt: $700-900 \mathrm{~m}$

Habitat:

W slopes of range, mixed ecotone of grasslands and short forest; arborescent associates include Grevillea, Helicia, Gardenia, Vaccinium, Eugenia; soils serpentine.

Field Char:

Short forest-margin tree $8 \mathrm{~m}$ tall, branching above and below; bole cylindrical, $15 \mathrm{~cm}$ diam.; bark brown, cracking-fissured vertically; leaves coriaceous; flowers pale yellow.

McDonald \& Ismail 3861

23 July 1993

\section{HERBARIUM BOGORIENSE HARVARD UNIVERSITY HERBARIA}




\section{PLANTS OF INDONESIA}

Proteaceae

Cat. \# 3862

\section{Helicia kjellbergii Burrett}

Det. By: McDonald 1993

Locality:

Kabaena; Kabaena,

Gunung Sabampolulu, $1 \mathrm{~km} \mathrm{S-SE} \mathrm{of} \mathrm{Tangkeno}$

$5^{\circ} 16^{\prime} \mathrm{S} 121^{\circ} 56^{\prime} \mathrm{E}$ Alt: $700-900 \mathrm{~m}$

Habitat:

W slopes of range, mixed ecotone of grasslands and short forest; arborescent associates include Grevillea, Helicia, Gardenia, Vaccinium, Eugenia; soils serpentine.

Field Char:

Common shrubby tree $7 \mathrm{~m}$ tall, rotund, branching at base and above; trunks to $10 \mathrm{~cm}$ diam.; bark tangray, smoothish, very shallow-fissured; foliage dense, leaves coriaceous, green above, greenglaucous below; fruits abundant, dark blue-purple, glaucous.

McDonald \& Ismail 3862

23 July 1993

\section{HERBARIUM BOGORIENSE HARVARD UNIVERSITY HERBARIA}

\section{PLANTS OF INDONESIA}

Celastraceae

Cat. \# 3863

\section{Salacia korthalsiana Miq.}

Det. By: McDonald 1993

Locality:

Kabaena; Kabaena,

Gunung Sabampolulu, $1 \mathrm{~km}$ S-SE of Tangkeno

$5^{\circ} 16^{\prime} \mathrm{S} 121^{\circ} 56^{\prime} \mathrm{E}$ Alt: $700-900 \mathrm{~m}$

Habitat:

W slopes of range, mixed ecotone of grasslands and short forest; arborescent associates include Grevillea, Helicia, Gardenia, Vaccinium, Eugenia; soils serpentine.

Field Char:

Clambering shrub in grasslands, branching abundantly; foliage light green, leaves coriaceous; flowers abundant, petals light green, glossy, basal disc dark green; ovary green, stigma orange; fruits green, hard.

McDonald \& Ismail 3863

HERBARIUM BOGORIENSE HARVARD UNIVERSITY HERBARIA 


\section{PLANTS OF INDONESIA}

Verbenaceae

Cat. \# 3864

Callicarpa pentandra roxb.

Det. By: McDonald 1993

Locality:

Kabaena; Kabaena,

Gunung Sabampolulu, $1 \mathrm{~km} \mathrm{S-SE} \mathrm{of} \mathrm{Tangkeno}$

$5^{\circ} 16^{\prime} \mathrm{S} 121^{\circ} 56^{\prime} \mathrm{E}$ Alt: $700-900 \mathrm{~m}$

Habitat:

W slopes of range, mixed ecotone of grasslands and short forest; arborescent associates include Grevillea, Helicia, Gardenia, Vaccinium, Eugenia; soils serpentine.

Field Char:

Forest-margin subcanopy tree $8 \mathrm{~m}$ tall, branching above; bole cylindrical, $15 \mathrm{~cm}$ diam.; bark gray, deeply fissured and plating; new growth stems rusty pubescent; calyx rusty pubescent, corolla pale lavender, anthers brown, style lavender, stigma white; berries bright pink when mature.

McDonald \& Ismail 3864

23 July 1993

\section{HERBARIUM BOGORIENSE HARVARD UNIVERSITY HERBARIA}

\section{PLANTS OF INDONESIA}

Apocynaceae

Cat. \# 3865

Alyxia clusiacea (Baill.) Pin.

Det. By: McDonald 1993

Locality:

Kabaena; Kabaena,

Gunung Sabampolulu, $1 \mathrm{~km}$ S-SE of Tangkeno

$5^{\circ} 16^{\prime} \mathrm{S} 121^{\circ} 56^{\prime} \mathrm{E}$ Alt: $700-900 \mathrm{~m}$

Habitat:

W slopes of range, mixed ecotone of grasslands and short forest; arborescent associates include Grevillea, Helicia, Gardenia, Vaccinium, Eugenia; soils serpentine.

Field Char:

Clambering liana to $10 \mathrm{~m}$ high; stems to $4 \mathrm{~cm}$ diam.; bark pale gray; leaves at stem apex only, latex white; calyx light green, corolla tube yellow, limb white.

McDonald \& Ismail 3865

23 July 1993

HERBARIUM BOGORIENSE HARVARD UNIVERSITY HERBARIA 


\section{PLANTS OF INDONESIA}

Olacaceae

Cat. \# 3866

Olax scandens Roxb.

Det. By: McDonald 1993

Locality:

Kabaena; Kabaena,

Gunung Sabampolulu, $1 \mathrm{~km}$ S-SE of Tangkeno

$5^{\circ} 16^{\prime} \mathrm{S} 121^{\circ} 56^{\prime} \mathrm{E}$ Alt: $700-900 \mathrm{~m}$

Habitat:

W slopes of range, mixed ecotone of grasslands and short forest; arborescent associates include Grevillea, Helicia, Gardenia, Vaccinium, Eugenia; soils serpentine.

Field Char: Clambering liana to $8 \mathrm{~m}$ high; stems stout, branching, to $6 \mathrm{~cm}$ diam. at base; bark gray smooth; bracteoles green; flowers devoid of perianth, ovary yellow, style cream; fruit immature, hard, cream.

McDonald \& Ismail 3866

23 July 1993

HERBARIUM BOGORIENSE HARVARD UNIVERSITY HERBARIA

\section{PLANTS OF INDONESIA}

Myristicaceae

Cat. \# 3867

Horsfieldia glabra (Bl.) Warb.

Det. By: McDonald 1993

Locality:

Kabaena; Kabaena,

Gunung Sabampolulu, $1 \mathrm{~km} \mathrm{S-SE} \mathrm{of} \mathrm{Tangkeno}$

$5^{\circ} 16^{\prime} \mathrm{S} 121^{\circ} 56^{\prime} \mathrm{E}$ Alt: $700-900 \mathrm{~m}$

Habitat:

W slopes of range, mixed ecotone of grasslands and short forest; arborescent associates include Grevillea, Helicia, Gardenia, Vaccinium, Eugenia; soils serpentine.

Field Char:

Uncommon forest-margin tree $9 \mathrm{~m}$ tall, branching midway up, lateral branches whorled; bole straight, cylindrical, $12 \mathrm{~cm}$ diam.; bark pale gray, narrowly grooved; leaves coriaceous; flower buds turquoise, yoke during anthesis.

McDonald \& Ismail 3867

23 July 1993

HERBARIUM BOGORIENSE HARVARD UNIVERSITY HERBARIA 


\section{PLANTS OF INDONESIA}

Myrsinaceae

Cat. \# 3868

Maesa cf. tetandra (Roxb.) DC.

Det. By: McDonald 1993

Locality:

Kabaena; Kabaena,

Gunung Sabampolulu, $1 \mathrm{~km}$ S-SE of Tangkeno

$5^{\circ} 16^{\prime} \mathrm{S} 121^{\circ} 56^{\prime} \mathrm{E}$ Alt: $700-900 \mathrm{~m}$

Habitat:

W slopes of range, mixed ecotone of grasslands and short forest; arborescent associates include Grevillea, Helicia, Gardenia, Vaccinium, Eugenia; soils serpentine.

Field Char:

Common climbing liana or shrub, 1-4 m tall, branching profusely; stems to $3 \mathrm{~cm}$ diam.;

inflorescence branching, yellowish, flowers cream.

McDonald \& Ismail 3868

23 July 1993

HERBARIUM BOGORIENSE HARVARD UNIVERSITY HERBARIA

PLANTS OF INDONESIA

Fabaceae

Cat. \# 3869

Albizia saponaria (Lour.) Bl.ex Miq.

Det. By: McDonald 1993

Locality:

Kabaena; Kabaena,

Gunung Sabampolulu, 1 km S-SE of Tangkeno

$5^{\circ} 16^{\prime} \mathrm{S} 121^{\circ} 56^{\prime} \mathrm{E}$ Alt: $700-900 \mathrm{~m}$

Habitat:

W slopes of range, mixed ecotone of grasslands and short forest; arborescent associates include Grevillea, Helicia, Gardenia, Vaccinium, Eugenia; soils serpentine.

Field Char:

Small tree at forest-margin, $10 \mathrm{~m}$ tall, branching above; bole crooked, $5 \mathrm{~cm}$ diam.; bark gray, smooth; leaves dark green, fruits light green.

McDonald \& Ismail 3869

23 July 1993

HERBARIUM BOGORIENSE HARVARD UNIVERSITY HERBARIA 


\section{PLANTS OF INDONESIA}

Euphorbiaceae

Cat. \# 3870

Glochidion aff. obscurum (Willd) Bl

Det. By: McDonald 1993

Locality:

Kabaena; Kabaena,

Gunung Sabampolulu, $1 \mathrm{~km}$ S-SE of Tangkeno

$5^{\circ} 16^{\prime} \mathrm{S} 121^{\circ} 56^{\prime} \mathrm{E}$ Alt: $700-900 \mathrm{~m}$

Habitat:

W slopes of range, mixed ecotone of grasslands and short forest; arborescent associates include Grevillea, Helicia, Gardenia, Vaccinium, Eugenia; soils serpentine.

Field Char:

Gnarled woody shrub $3 \mathrm{~m}$ tall and wide, branching abundantly, trunks to $5 \mathrm{~cm}$ diam.; bark brown-gray, cracked, exfoliating; foliage at stem apex, leaves coriaceous; flowers cream, waxy coriaceous; fruits depressed, subrotund, fleshy, white-striped pink.

McDonald \& Ismail 3870

24 July 1993

\section{HERBARIUM BOGORIENSE HARVARD UNIVERSITY HERBARIA}

\section{PLANTS OF INDONESIA}

Myrtaceae

Cat. \# 3871

Leptospermum amboinse $\mathrm{Bl}$.

Det. By: McDonald 1993

Locality:

Kabaena; Kabaena,

Gunung Sabampolulu, $1 \mathrm{~km}$ S-SE of Tangkeno

$5^{\circ} 16^{\prime} \mathrm{S} 121^{\circ} 56^{\prime} \mathrm{E}$ Alt: $700-900 \mathrm{~m}$

Habitat:

W slopes of range, mixed ecotone of grasslands and short forest; arborescent associates include Grevillea, Helicia, Gardenia, Vaccinium, Eugenia; soils serpentine.

Field Char:

Locally dominant tree $5 \mathrm{~m}$ tall, branching midway up; bole contoured-grooved, $30 \mathrm{~cm}$ diam.; bark palegray, deeply fissure-furrowed; lateral branches contorted, bearing foliage at terminus; leaves coriaceous; flowers occasional, petals white, calyx green; fruit brown, woody.

McDonald \& Ismail 3871 


\section{PLANTS OF INDONESIA}

Cat. \# 3872

Zizygium fastigiatum (Bl.) Merr.

Det. By: McDonald 1993

Locality:

Kabaena; Kabaena,

Gunung Sabampolulu, $1 \mathrm{~km} \mathrm{S-SE}$ of Tangkeno

$5^{\circ} 16^{\prime} \mathrm{S} 121^{\circ} 56^{\prime} \mathrm{E}$ Alt: $700-900 \mathrm{~m}$

Habitat:

W slopes of range, mixed ecotone of grasslands and short forest; arborescent associates include Grevillea, Helicia, Gardenia, Vaccinium, Eugenia; soils serpentine.

Field Char:

Common canopy tree $15 \mathrm{~m}$ tall, branching above; bole striaght, cylindrical, $18 \mathrm{~cm}$ diam.; bark palid gray, smoothish, exfoliating in roundish flakes; leaves coriaceous; flowers abundant, apetalous, white, yellowish inside calyx cup.

McDonald \& Ismail 3872

24 July 1993

\section{HERBARIUM BOGORIENSE HARVARD UNIVERSITY HERBARIA}

\section{PLANTS OF INDONESIA}

Myristicaceae

Cat. \# 3873

Myristica cf. koordersii Warb.

Det. By: McDonald 1993

Locality:

Kabaena; Kabaena,

Gunung Sabampolulu, $1 \mathrm{~km} \mathrm{S-SE} \mathrm{of} \mathrm{Tangkeno}$

$5^{\circ} 16^{\prime} \mathrm{S} 121^{\circ} 56^{\prime} \mathrm{E}$ Alt: $700-900 \mathrm{~m}$

Habitat:

W slopes of range, mixed ecotone of grasslands and short forest; arborescent associates include Grevillea, Helicia, Gardenia, Vaccinium, Eugenia; soils serpentine.

Field Char:

Canopy tree $12 \mathrm{~m}$ tall, branching above; bole straight, cylindrical, $15 \mathrm{~cm}$ diam.; bark dark brown, rough and sparingly vertically cracked; lateral branches perpendicular to trunk and pendant; leaves dark green above, silvery-green below, coriaceous; fruit orange-tan.

McDonald \& Ismail 3873

24 July 1993

HERBARIUM BOGORIENSE HARVARD UNIVERSITY HERBARIA 


\section{PLANTS OF INDONESIA}

Magnoliaceae

Cat. \# 3874

Magnolia candollii (Bl.) Keng var. candollii

Det. By: McDonald 1993

Locality:

Kabaena; Kabaena,

Gunung Sabampolulu, $1 \mathrm{~km}$ S-SE of Tangkeno

$5^{\circ} 16^{\prime} \mathrm{S} 121^{\circ} 56^{\prime} \mathrm{E}$ Alt: $700-900 \mathrm{~m}$

Habitat:

W slopes of range, mixed ecotone of grasslands and short forest; arborescent associates include Grevillea, Helicia, Gardenia, Vaccinium, Eugenia; soils serpentine.

Field Char:

Canopy tree $18 \mathrm{~m}$ tall, branching midway up; bole straight, cylindrical, $20 \mathrm{~cm}$ diam.; bark tan-gray, rough, inner bark tan, new growth stems green, old growth stems gray; leaves coriaceous; flower buds ellipsoid, green; sepals 3, green, coriaceous, reflexed during anthesis; petals waxy.

McDonald \& Ismail 3874

24 July 1993

\section{HERBARIUM BOGORIENSE \\ HARVARD UNIVERSITY HERBARIA}

\section{PLANTS OF INDONESIA}

Aquifoliaceae

Cat. \# 3875

Ilex $s p$.

Det. By: McDonald 1993

Locality:

Kabaena; Kabaena,

Gunung Sabampolulu, $1 \mathrm{~km} \mathrm{S-SE} \mathrm{of} \mathrm{Tangkeno}$

$5^{\circ} 16^{\prime} \mathrm{S} 121^{\circ} 56^{\prime} \mathrm{E}$ Alt: $700-900 \mathrm{~m}$

Habitat:

W slopes of range, mixed ecotone of grasslands and short forest; arborescent associates include Grevillea, Helicia, Gardenia, Vaccinium, Eugenia; soils serpentine.

Field Char:

Canopy tree $26 \mathrm{~m}$ tall, branching above; bole somewhat crooked, cylindrical, $25 \mathrm{~cm}$ diam.; bark tan-gray, rough, spongy inside; leaves light green; fruits immature, light green with brown apex.

McDonald \& Ismail 3875

HERBARIUM BOGORIENSE HARVARD UNIVERSITY HERBARIA 


\section{PLANTS OF INDONESIA}

Celastraceae

Cat. \# 3876

Euonymus impressus Blakelock

Det. By: McDonald 1993

Locality:

Kabaena; Kabaena,

Gunung Sabampolulu, $1 \mathrm{~km}$ S-SE of Tangkeno

$5^{\circ} 16^{\prime} \mathrm{S} 121^{\circ} 56^{\prime} \mathrm{E}$ Alt: $700-900 \mathrm{~m}$

Habitat:

W slopes of range, mixed ecotone of grasslands and short forest; arborescent associates include Grevillea, Helicia, Gardenia, Vaccinium, Eugenia; soils serpentine.

Field Char:

Midstory tree $8 \mathrm{~m}$ tall, branching midway up; bole somewhat 3-angular, $10 \mathrm{~cm}$ diam.; bark gray, rough, leaves light green; disk and calyx reddish, corolla cream, petals $4 \mathrm{~mm}$ long, notched at apex; gynoecium green, flat; fruit obpyriform, green turning red, dehiscing along 2-5 valves.

McDonald \& Ismail 3876

24 July 1993

HERBARIUM BOGORIENSE HARVARD UNIVERSITY HERBARIA

\section{PLANTS OF INDONESIA}

Celastraceae

Cat. \# 3877

Euonymus javanicus $B l$.

Det. By: McDonald 1993

Locality:

Kabaena; Kabaena,

Gunung Sabampolulu, $1 \mathrm{~km}$ S-SE of Tangkeno

$5^{\circ} 16^{\prime} \mathrm{S} 121^{\circ} 56^{\prime} \mathrm{E}$ Alt: $700-900 \mathrm{~m}$

Habitat:

W slopes of range, mixed ecotone of grasslands and short forest; arborescent associates include Grevillea, Helicia, Gardenia, Vaccinium, Eugenia; soils serpentine.

Field Char:

Midstory tree $10 \mathrm{~m}$ tall, branching above; trunk straight, cylindrical, $10 \mathrm{~cm}$ diam.; bark gray, narrowly grooved; leaves light green, coriaceous; calyx greenish, calyx 5, revolute medially, fringed at apex, bright maroon proximally, light green distally, disk light green. 


\section{PLANTS OF INDONESIA}

Theaceae

Cat. \# 3878

Adinandra sp. nov.?

Det. By: McDonald 1993

Locality:

Kabaena; Kabaena,

Gunung Sabampolulu, $1 \mathrm{~km} \mathrm{S-SE} \mathrm{of} \mathrm{Tangkeno}$

$5^{\circ} 16^{\prime} \mathrm{S} 121^{\circ} 56^{\prime} \mathrm{E}$ Alt: $700-900 \mathrm{~m}$

Habitat:

W slopes of range, mixed ecotone of grasslands and short forest; arborescent associates include Grevillea, Helicia, Gardenia, Vaccinium, Eugenia; soils serpentine.

Field Char:

Uncommon canopy tree, $20 \mathrm{~m}$ tall; branching above and below; bole cylindrical, straight, $1 \mathrm{~m}$ diam.; bark dark brown, deeply furrowed and plated; leaves coriaceous; floral buds rotund, pale salmon; fruits immature, green.

McDonald \& Ismail 3878

24 July 1993

\section{HERBARIUM BOGORIENSE HARVARD UNIVERSITY HERBARIA}

\section{PLAN'TS OF INDONESIA}

Arecaceae

Cat. \# 3879

Caryotasp.

Det. By: McDonald 1993

Locality:

Kabaena; Kabaena,

Gunung Sabampolulu, $1 \mathrm{~km}$ S-SE of Tangkeno

$5^{\circ} 16^{\prime} \mathrm{S} 121^{\circ} 56^{\prime} \mathrm{E}$ Alt: $700-900 \mathrm{~m}$

Habitat:

W slopes of range, mixed ecotone of grasslands and short forest; arborescent associates include Grevillea, Helicia, Gardenia, Vaccinium, Eugenia; soils serpentine.

Field Char:

Uncommon understory palm, $5 \mathrm{~m}$ tall; bole $6 \mathrm{~cm}$ diam.; leaves 4-5 cm long; inflorescence ca. 50 pendent branchlets; fruits immature, green.

McDonald \& Ismail 3879

24 July 1993

HERBARIUM BOGORIENSE HARVARD UNIVERSITY HERBARIA 


\section{PLANTS OF INDONESIA}

Casuarinaceae

Cat. \# 3880

\section{Gymnostoma sumatrana}

Det. By: McDonald 1993

Locality:

Kabaena; Kabaena,

Gunung Sabampolulu, $1 \mathrm{~km}$ S-SE of Tangkeno

$5^{\circ} 16^{\prime} \mathrm{S} 121^{\circ} 56^{\prime} \mathrm{E}$ Alt: $700-900 \mathrm{~m}$

Habitat:

W slopes of range, mixed ecotone of grasslands and short forest; arborescent associates include Grevillea, Helicia, Gardenia, Vaccinium, Eugenia; soils serpentine.

Field Char:

Timberline, canopy tree, $12 \mathrm{~m}$ tall, branching above and sparingly; bole straight, cylindrical, $12 \mathrm{~cm}$ diam. ; bark light gray, rough; foliage glossy-green, leaves coriaceous; flower buds in 3 rows of 3 flowers, calyx 4-merous, light green, petals 4 , white, coriaceous, stamens opposite.

McDonald \& Ismail 3880

25 July 1993

\section{HERBARIUM BOGORIENSE HARVARD UNIVERSITY HERBARIA}

\section{PLANTS OF INDONESIA}

Cat. \# 3881

Evodia eunera Miq.

Det. By: McDonald 1993

Locality:

Kabaena; Kabaena,

Gunung Sabampolulu, $1 \mathrm{~km} \mathrm{S-SE} \mathrm{of} \mathrm{Tangkeno}$

$5^{\circ} 16^{\prime} \mathrm{S} 121^{\circ} 56^{\prime} \mathrm{E}$ Alt: $700-900 \mathrm{~m}$

Habitat:

W slopes of range, mixed ecotone of grasslands and short forest; arborescent associates include Grevillea, Helicia, Gardenia, Vaccinium, Eugenia; soils serpentine.

Field Char:

Canopy tree $12 \mathrm{~m}$ tall, branching above; trunk straight, cylindrical, $12 \mathrm{~cm}$ diam; bark light gray, rough; branching sparing, foliage glossy green; calyx 4-merous, light green, petals 4, white, coriaceous, stamens opposite petals, ovary 4-lobed, green, style white.

Notes:

NCI voucher

McDonald \& Ismail 3881 


\section{PLANTS OF INDONESIA}

Elaeocarpaceae

Cat. \# 3882

Elaeocarpus aff. griffith (Wight) A Gray

Det. By: McDonald 1993

Locality:

Kabaena; Kabaena,

Gunung Sabampolulu, $1 \mathrm{~km} \mathrm{S-SE} \mathrm{of} \mathrm{Tangkeno}$

$5^{\circ} 16^{\prime} \mathrm{S} 121^{\circ} 56^{\prime} \mathrm{E}$ Alt: $700-900 \mathrm{~m}$

Habitat:

W slopes of range, mixed ecotone of grasslands and short forest; arborescent associates include Grevillea, Helicia, Gardenia, Vaccinium, Eugenia; soils serpentine.

Field Char:

Short tree $4 \mathrm{~m}$ tall, branching midway up; trunk cylindrical, $3 \mathrm{~cm}$ diam.; bark pale gray; calyx vary pale green, petals white, fringed, ca. $8 \mathrm{~mm}$ long; stamens numerous, with small yellow glands at base collaring the green ovary; fruits dark blue-green, glossy.

McDonald \& Ismail 3882

25 July 1993

\section{HERBARIUM BOGORIENSE \\ HARVARD UNIVERSITY HERBARIA}

\section{PLANTS OF INDONESIA}

Oleaceae

Cat. \# 3883

\section{Ligustrum sp.}

Det. By: McDonald 1993

Locality:

Kabaena; Kabaena,

Gunung Sabampolulu, $1 \mathrm{~km} \mathrm{S-SE} \mathrm{of} \mathrm{Tangkeno}$

$5^{\circ} 16^{\prime} \mathrm{S} 121^{\circ} 56^{\prime} \mathrm{E}$ Alt: $700-900 \mathrm{~m}$

Habitat:

W slopes of range, mixed ecotone of grasslands and short forest; arborescent associates include Grevillea, Helicia, Gardenia, Vaccinium, Eugenia; soils serpentine.

Field Char:

Occasional shrub 2-3 $\mathrm{m}$ tall, branching above and at base; basal stems to $3 \mathrm{~cm}$ diam.; new growth reddishbrown with tan lenticels, bark light gray; leaves coriaceous, green above, light green below; fruits abundant, green below, dark purple below .

McDonald \& Ismail 3883 


\section{PLANTS OF INDONESIA}

Proteaceae

Cat. \# 3884

Macadamia sp. nov. McDonald ined.

Det. By: McDonald 1993

Locality:

Kabaena; Kabaena,

Gunung Sabampolulu, $1 \mathrm{~km}$ S-SE of Tangkeno

$5^{\circ} 16^{\prime} \mathrm{S} 121^{\circ} 56^{\prime} \mathrm{E}$ Alt: $700-900 \mathrm{~m}$

Habitat:

W slopes of range, mixed ecotone of grasslands and short forest; arborescent associates include Grevillea, Helicia, Gardenia, Vaccinium, Eugenia; soils serpentine.

Field Char:

Canopy tree $14 \mathrm{~m}$ tall, branching above; bole straight, cylindrical, $70 \mathrm{~cm}$ diam.; bark brown, somewhat flaky; leaves coriaceous; fruit single, hard, green.

McDonald \& Ismail 3884

25 July 1993

HERBARIUM BOGORIENSE HARVARD UNIVERSITY HERBARIA

\section{PLANTS OF INDONESIA}

Lauraceae

Cat. \# 3885

Cryptocarya parviflora Merr.

Det. By: McDonald 1993

Locality:

Kabaena; Kabaena,

Gunung Sabampolulu, $1 \mathrm{~km}$ S-SE of Tangkeno

$5^{\circ} 16^{\prime}$ 'S $121^{\circ} 56^{\prime}$ E Alt: $700-900 \mathrm{~m}$

Habitat:

W slopes of range, mixed ecotone of grasslands and short forest; arborescent associates include Grevillea, Helicia, Gardenia, Vaccinium, Eugenia; soils serpentine.

Field Char:

Canopy tree $12 \mathrm{~m}$ tall, canopy "krummholz"; bole straight, cylindrical, $20 \mathrm{~cm}$ diam.; bark gray, rough; foliage dark green; leaves coriaceous; fruits immature, green.

McDonald \& Ismail 3885

HERBARIUM BOGORIENSE HARVARD UNIVERSITY HERBARIA 


\section{PLANTS OF INDONESIA}

Theaceae

Cat. \# 3886

Ternstroemia sp.

Det. By: McDonald 1993

Locality:

Kabaena; Kabaena,

Gunung Sabampolulu, $1 \mathrm{~km} \mathrm{S-SE} \mathrm{of} \mathrm{Tangkeno}$

$5^{\circ} 16^{\prime} \mathrm{S} 121^{\circ} 56^{\prime} \mathrm{E}$ Alt: $700-900 \mathrm{~m}$

Habitat:

W slopes of range, mixed ecotone of grasslands and short forest; arborescent associates include Grevillea, Helicia, Gardenia, Vaccinium, Eugenia; soils serpentine.

Field Char:

Midstory tree $8 \mathrm{~m}$ tall, branching above; bole cylindrical, $8 \mathrm{~cm}$ diam.; bark light gray, rough; foliage light green, leves coriaceous, green with reddish margins; calyx reddish, corolla $8 \mathrm{~mm}$ diam., petals cream, androecium yellow; fruits reddish and green.

McDonald \& Ismail 3886

25 July 1993

\section{HERBARIUM BOGORIENSE \\ HARVARD UNIVERSITY HERBARIA}

\section{PLANTS OF INDONESIA}

Cat. \# 3887

Planchonella sp.

Det. By: McDonald 1993

Locality:

Kabaena; Kabaena,

Gunung Sabampolulu, $1 \mathrm{~km}$ S-SE of Tangkeno

$5^{\circ} 16^{\prime} \mathrm{S} 121^{\circ} 56^{\prime} \mathrm{E}$ Alt: $700-900 \mathrm{~m}$

Habitat:

$\mathrm{W}$ slopes of range, mixed ecotone of grasslands and short forest; arborescent associates include Grevillea, Helicia, Gardenia, Vaccinium, Eugenia; soils serpentine.

Field Char:

Tree $13 \mathrm{~m}$ tall, branching midway up; trunk straight, cylindrical, $20 \mathrm{~cm}$ diam.; bark dark brown, cracking into hard plates; leaves terminal, green above, light green below; flowers solitary in leaf axils, peduncles green or rusty, clayx light green.

McDonald \& Ismail 3887

25 July 1993

HERBARIUM BOGORIENSE HARVARD UNIVERSITY HERBARIA 


\section{PLANTS OF INDONESIA}

Arecaceae

Cat. \# 3888

Pinanga sp.

Det. By: McDonald 1993

Locality:

Kabaena; Kabaena,

Gunung Sabampolulu, $1 \mathrm{~km}$ S-SE of Tangkeno

$5^{\circ} 16^{\prime} \mathrm{S} 121^{\circ} 56^{\prime} \mathrm{E}$ Alt: $700-900 \mathrm{~m}$

Habitat:

W slopes of range, mixed ecotone of grasslands and short forest; arborescent associates include Grevillea, Helicia, Gardenia, Vaccinium, Eugenia; soils serpentine.

Field Char:

Understory tree, in ravines, solitary or colonial, aerial roots $2-5 \mathrm{~m}$ long; fronds $2-2.5 \mathrm{~m}$ long, 1-1.5 $\mathrm{m}$ wide, sheath yellowish; inflorescence creamypeach, flowers cream; fruits immature, green.

McDonald \& Ismail 3888

25 July 1993

HERBARIUM BOGORIENSE HARVARD UNIVERSITY HERBARIA

\section{PLANTS OF INDONESIA}

Oleaceae

Cat. \# 3889

Chionanthus aff. ramiflorus Roxb.

Det. By: McDonald 1993

Locality:

Kabaena; Kabaena,

Gunung Sabampolulu, $1 \mathrm{~km}$ S-SE of Tangkeno

$5^{\circ} 16^{\prime} \mathrm{S} 121^{\circ} 56^{\prime} \mathrm{E}$ Alt: $700-900 \mathrm{~m}$

Habitat:

W slopes of range, mixed ecotone of grasslands and short forest; arborescent associates include Grevillea, Helicia, Gardenia, Vaccinium, Eugenia; soils serpentine.

Field Char:

Canopy tree in ravine, $24 \mathrm{~m}$ tall, branching above; bole cylindrical, $18 \mathrm{~cm}$ diam.; bark light gray, somewhat roughened; fruits immature, green.

McDonald \& Ismail 3889

25 July 1993

HERBARIUM BOGORIENSE HARVARD UNIVERSITY HERBARIA 


\section{PLANTS OF INDONESIA}

Clusiaceae

Cat. \# 3890

Calophyllum soulatri Burm. $f$.

Det. By: McDonald 1993

Locality:

Kabaena; Kabaena,

Gunung Sabampolulu, $1 \mathrm{~km} \mathrm{S-SE} \mathrm{of} \mathrm{Tangkeno}$

$5^{\circ} 16^{\prime} \mathrm{S} 121^{\circ} 56^{\prime} \mathrm{E}$ Alt: $700-900 \mathrm{~m}$

Habitat:

W slopes of range, mixed ecotone of grasslands and short forest; arborescent associates include Grevillea, Helicia, Gardenia, Vaccinium, Eugenia; soils serpentine.

Field Char:

Midstory tree $12 \mathrm{~m}$ tall, branching midway up; bole cylindrical, $8 \mathrm{~cm}$ diam.; bark gray, smoothish.

McDonald \& Ismail 3890

25 July 1993

\section{HERBARIUM BOGORIENSE \\ HARVARD UNIVERSITY HERBARIA}

\section{PLANTS OF INDONESIA}

Cat. \# 3891

\section{Cyathea aff. elmeri Copel}

Det. By: McDonald 1993

Locality:

Kabaena; Kabaena,

Gunung Sabampolulu, $1 \mathrm{~km}$ S-SE of Tangkeno

$5^{\circ} 16^{\prime} \mathrm{S} 121^{\circ} 56^{\prime} \mathrm{E}$ Alt: $700-900 \mathrm{~m}$

Habitat:

W slopes of range, mixed ecotone of grasslands and short forest; arborescent associates include Grevillea, Helicia, Gardenia, Vaccinium, Eugenia; soils serpentine.

Field Char:

Arborescent fern in samll ravines, 1-2 m tall, stems monopodial.

Notes:

NCI voucher

McDonald \& Ismail 3891

HERBARIUM BOGORIENSE HARVARD UNIVERSITY HERBARIA 


\section{PLANTS OF INDONESIA}

Ulmaceae

Cat. \# 3892

Trema orientalis (L.) Bl.

Det. By: McDonald 1993

Locality:

Kabaena; Kabaena,

Gunung Sabampolulu, $1 \mathrm{~km} \mathrm{S-SE} \mathrm{of} \mathrm{Tangkeno}$

$5^{\circ} 16^{\prime} \mathrm{S} 121^{\circ} 56^{\prime} \mathrm{E}$ Alt: $700-900 \mathrm{~m}$

Habitat:

W slopes of range, mixed ecotone of grasslands and short forest; arborescent associates include Grevillea, Helicia, Gardenia, Vaccinium, Eugenia; soils serpentine.

Field Char:

Canopy tree $23 \mathrm{~m}$ tall, branching above and below; bole cylindrical, $70 \mathrm{~cm}$ diam., wood soft; bark light gray, smoothish; flowers apetalous, ovary green, stigma white.

McDonald \& Ismail 3892

26 July 1993

HERBARIUM BOGORIENSE

HARVARD UNIVERSITY HERBARIA

\section{PLANTS OF INDONESIA}

Lauraceae

Cat. \# 3893

Dehaasia caesia bl.

Det. By: McDonald 1993

Locality:

Kabaena; Kabaena,

Gunung Sabampolulu, $1 \mathrm{~km} \mathrm{S-SE} \mathrm{of} \mathrm{Tangkeno}$

$5^{\circ} 16^{\prime} \mathrm{S} 121^{\circ} 56^{\prime} \mathrm{E}$ Alt: $700-900 \mathrm{~m}$

Habitat:

W slopes of range, mixed ecotone of grasslands and short forest; arborescent associates include Grevillea, Helicia, Gardenia, Vaccinium, Eugenia; soils serpentine.

Field Char:

Canopy tree $12 \mathrm{~m}$ tall, branching above; bole gray, weakly vertical-furrowed; foliage coriaceous, light green; flowers light green, stamens yellow.

McDonald \& Ismail 3893

26 July 1993

HERBARIUM BOGORIENSE HARVARD UNIVERSITY HERBARIA 


\section{PLANTS OF INDONESIA}

Lauraceae

Cat. \# 3894

Dehaasia sp.

Det. By: McDonald 1993

Locality:

Kabaena; Kabaena,

Gunung Sabampolulu, $1 \mathrm{~km}$ S-SE of Tangkeno

$5^{\circ} 16^{\prime} \mathrm{S} 121^{\circ} 56^{\prime} \mathrm{E}$ Alt: $700-900 \mathrm{~m}$

Habitat:

W slopes of range, mixed ecotone of grasslands and short forest; arborescent associates include Grevillea, Helicia, Gardenia, Vaccinium, Eugenia; soils serpentine.

Field Char:

Canopy tree $20 \mathrm{~m}$ tall, branching midway up; bole straight, cylindrical, $70 \mathrm{~cm}$ diam.; bark brown, deeply furrowed; leaves dark green, coriaceous; immature fruits light green, at maturity peduncles red, fruits black.

McDonald \& Ismail 3894

26 July 1993

\section{HERBARIUM BOGORIENSE HARVARD UNIVERSITY HERBARIA}

\section{PLANTS OF INDONESIA}

Myristicaceae

Cat. \# 3895

Knema tomentella (Miq.) Warb.

Det. By: McDonald 1993

Locality:

Kabaena; Kabaena,

Gunung Sabampolulu, $1 \mathrm{~km}$ S-SE of Tangkeno

$5^{\circ} 16^{\prime} \mathrm{S} 121^{\circ} 56^{\prime} \mathrm{E}$ Alt: $700-900 \mathrm{~m}$

Habitat:

W slopes of range, mixed ecotone of grasslands and short forest; arborescent associates include Grevillea, Helicia, Gardenia, Vaccinium, Eugenia; soils serpentine.

Field Char:

Midstory tree $11 \mathrm{~m}$ tall, branching above and below; bole cylindrical, $30 \mathrm{~cm}$ diam.; bark grayish-brown, smooth; lateral branches perpendicular to bole, then pendent; leaves green above, silverish below; fruits brown.

McDonald \& Ismail 3895

26 July 1993

\section{HERBARIUM BOGORIENSE HARVARD UNIVERSITY HERBARIA}




\section{PLANTS OF INDONESIA}

Annonaceae

Cat. \# 3896

Annonaceae Goniothalamus sp.

det. S.Zmarty \& P. Byglave (K) 1997

Det. By: MeDenald 1993

Locality:

Kabaena; Kabaena,

Gunung Sabampolulu, $1 \mathrm{~km} \mathrm{S-SE} \mathrm{of} \mathrm{Tangkeno}$

$5^{\circ} 16^{\prime} \mathrm{S} 121^{\circ} 56^{\prime} \mathrm{E}$ Alt: $700-900 \mathrm{~m}$

Habitat:

W slopes of range, mixed ecotone of grasslands and short forest; arborescent associates include Grevillea, Helicia, Gardenia, Vaccinium, Eugenia; soils serpentine.

Field Char:

Common understory tree $5 \mathrm{~m}$ tall; trunk $4 \mathrm{~cm}$ diam.; inflorescence from base of trunk; lower tepals pale green, pink at base, inner tepals pinkish; androecium pink, carpels cream, tepals turning pale-maroon; fruits immature, dark olive, subcorky-striate longitudinally.

McDonald \& Ismail 3896

26 July 1993

\section{HERBARIUM BOGORIENSE HARVARD UNIVERSITY HERBARIA}

\section{PLANTS OF INDONESIA}

Ebenaceae

Cat. \# 3897

Diospyros sp.

Det. By: McDonald 1993

Locality:

Kabaena; Kabaena,

Gunung Sabampolulu, $1 \mathrm{~km}$ S-SE of Tangkeno

$5^{\circ} 16^{\prime} \mathrm{S} 121^{\circ} 56^{\prime} \mathrm{E}$ Alt: $700-900 \mathrm{~m}$

Habitat:

W slopes of range, mixed ecotone of grasslands and short forest; arborescent associates include Grevillea, Helicia, Gardenia, Vaccinium, Eugenia; soils serpentine.

Field Char:

Short canopy tree $13 \mathrm{~m}$ tall, branching above; bole somewhat crooked, $15 \mathrm{~cm}$. diam.; bark grayish, smoothish, inner bark yellow; leaves dark green above, pale green below; flowers cauliflorous on lateral branches; maturing fruits silvery green velutinous.

McDonald \& Ismail 3897 


\section{PLANTS OF INDONESIA}

Ulmaceae

Cat. \# 3898

Gironniera subaequalis Planch.

Det. By: McDonald 1993

Locality:

Kabaena; Kabaena,

Gunung Sabampolulu, $1 \mathrm{~km} \mathrm{S-SE} \mathrm{of} \mathrm{Tangkeno}$

$5^{\circ} 16^{\prime} \mathrm{S} 121^{\circ} 56^{\prime} \mathrm{E}$ Alt: $700-900 \mathrm{~m}$

Habitat:

W slopes of range, mixed ecotone of grasslands and short forest; arborescent associates include Grevillea, Helicia, Gardenia, Vaccinium, Eugenia; soils serpentine.

Field Char:

Canopy tree $22 \mathrm{~m}$ tall, branching midway up; bole straight, cylindrical, $60 \mathrm{~cm}$ diam.; bark light gray, rough; leaves dark green above, light green below; fruits abundant, dull orange.

McDonald \& Ismail 3898

26 July 1993

HERBARIUM BOGORIENSE

HARVARD UNIVERSITY HERBARIA

PLANTS OF INDONESIA

Burseraceae

Cat. \# 3899

Canarium hirsutum Willd. Leeuh. multicostulatum

Det. By: McDonald 1993

Locality:

Kabaena; Kabaena,

Gunung Sabampolulu, $1 \mathrm{~km} \mathrm{S-SE} \mathrm{of} \mathrm{Tangkeno}$

$5^{\circ} 16^{\prime} \mathrm{S} 121^{\circ} 56^{\prime} \mathrm{E}$ Alt: $700-900 \mathrm{~m}$

Habitat:

W slopes of range, mixed ecotone of grasslands and short forest; arborescent associates include Grevillea, Helicia, Gardenia, Vaccinium, Eugenia; soils serpentine.

Field Char:

Canopy tree $18 \mathrm{~m}$ tall, branching above; bole straight, cylindrical, $20 \mathrm{~cm}$ diam.; bark pale gray, rough, inner bark tan; stems resinous, aromatic; fruits occasional, turning green to black.

McDonald \& Ismail 3899

HERBARIUM BOGORIENSE HARVARD UNIVERSITY HERBARIA 


\title{
PLANTS OF INDONESIA
}

Lauraceae

Cat. \# 4000

Lindera malaccensis Hook. $f$.

Det. By: McDonald 1993

Locality:

Kabaena; Kabaena,

Gunung Sabampolulu, $1 \mathrm{~km} \mathrm{S-SE} \mathrm{of} \mathrm{Tangkeno}$

$5^{\circ} 16^{\prime} \mathrm{S} 121^{\circ} 56^{\prime} \mathrm{E}$ Alt: $700-900 \mathrm{~m}$

Habitat:

W slopes of range, mixed ecotone of grasslands and short forest; arborescent associates include Grevillea, Helicia, Gardenia, Vaccinium, Eugenia; soils serpentine.

Field Char:

Canopy tree $18 \mathrm{~m}$ tall, branching midway up; bole somewhat buttressed, $40 \mathrm{~cm}$ diam.; bark reddish brown, crust under plates; leaves dark green above, light green below; stamens in 5 fascicles, anthers yellow.

McDonald \& Ismail 4000

26 July 1993

\author{
HERBARIUM BOGORIENSE \\ HARVARD UNIVERSITY HERBARIA
}

\section{PLANTS OF INDONESIA}

Sterculiaceae

Cat. \# 4001

\section{Melochia arborea Blanco}

Det. By: McDonald 1993

Locality:

Kabaena; Kabaena,

Gunung Sabampolulu, $1 \mathrm{~km} \mathrm{S-SE} \mathrm{of} \mathrm{Tangkeno}$

$5^{\circ} 16^{\prime}$ 'S $121^{\circ} 56^{\prime}$ E Alt: $700-900 \mathrm{~m}$

\section{Habitat:}

W slopes of range, mixed ecotone of grasslands and short forest; arborescent associates include Grevillea, Helicia, Gardenia, Vaccinium, Eugenia; soils serpentine.

Field Char:

Canopy tree $12 \mathrm{~m}$ tall, branching above and below; bole cylindrical, $20 \mathrm{~cm}$ diam.; bark gray with horizontal, black lenticels; calyx pale green, petals pink, corolla throat orange; filaments lavender.

McDonald \& Ismail 4001

HERBARIUM BOGORIENSE HARVARD UNIVERSITY HERBARIA 


\section{PLANTS OF INDONESIA}

Icacinaceae

Cat. \# 4002

Gomphandra sp.

Det. By: McDonald 1993

Locality:

Kabaena; Kabaena,

Gunung Sabampolulu, $1 \mathrm{~km} \mathrm{S-SE} \mathrm{of} \mathrm{Tangkeno}$

$5^{\circ} 16^{\prime} \mathrm{S} 121^{\circ} 56^{\prime} \mathrm{E}$ Alt: $700-900 \mathrm{~m}$

Habitat:

W slopes of range, mixed ecotone of grasslands and short forest; arborescent associates include Grevillea, Helicia, Gardenia, Vaccinium, Eugenia; soils serpentine.

Field Char:

Canopy tree $15 \mathrm{~m}$ tall, branching midway up; bole straight, cylindrical, $50 \mathrm{~cm}$ diam.; bark light gray, patchy-tan; foliage green-glossy; fruits abundant, light green, immature.

McDonald \& Ismail 4002

26 July 1993

\section{HERBARIUM BOGORIENSE \\ HARVARD UNIVERSITY HERBARIA}

\section{PLANTS OF INDONESIA}

Fabaceae

Cat. \# 4003

\section{Mucuna sp.}

Det. By: McDonald 1993

Locality:

Kabaena; Kabaena,

Gunung Sabampolulu, $1 \mathrm{~km}$ S-SE of Tangkeno

$5^{\circ} 16^{\prime} \mathrm{S} 121^{\circ} 56^{\prime} \mathrm{E}$ Alt: $700-900 \mathrm{~m}$

Habitat:

W slopes of range, mixed ecotone of grasslands and short forest; arborescent associates include Grevillea, Helicia, Gardenia, Vaccinium, Eugenia; soils serpentine.

Field Char:

Robust liana branching prodigiously, lower stems to $3 \mathrm{~cm}$ diam, lvs dark green-glossy; infl. and calyx light green, corolla light green with dark purple throat, whitish androecial tube, ovary yellowishpubescent.

McDonald \& Ismail 4003 


\section{PLANTS OF}

This is a voucher specimen for anticancer testing: YES NO

Fam.: Pittosporaceae

Det. by:

$$
\text { Pittosporum }
$$

Field characters:

rean

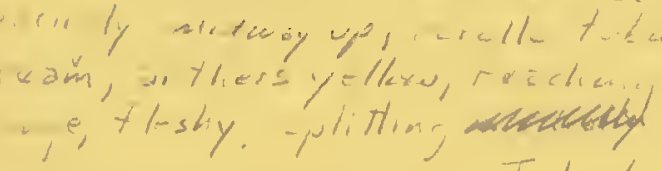

Loc: Kabaena Islant, Gunuarg Sabampolulu, $1 \mathrm{~km}$ S-SE of Tongkeno.

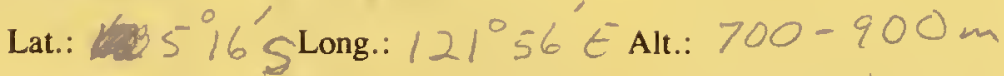

Habitat: $W$ slopes of range, mixed ecotone of grasslands and short forest. Aiborescent asscciates include Grevillea, Helicia, Gardenia, Vacoiniom Solls serpentine.

Loc. name(s): "Kayu tei ayam" (Fish-chicken wood)

Med. use(s):

Part(s) used:

General notes:

Hazard notes:

Collector(s): Mc Donald $\sum_{1}^{\prime}$ Ism

$$
\text { No.: } 3842 \text { Date: } 21 \text { Vul } 1993
$$

Sample plant part(s): $\quad L F U-14 Z-7931-\omega$

NCI code number(s): TW $\quad-7932-X$

Collected under the auspices of:

U.S. National Cancer Institute, Bethesda, MD.

University of Illinois at Chicago, Chicago, IL.

-Arnold Arboretum, Harvard University, Cambridge, MA.

Field Museum of Natural History, Chicago, IL.

Host Institution: $B, Y / F|P|$ 


\section{PLANTS OF}

This is a voucher specimen for anticancer testing: YES NO

Fam.: Epacridaceae

$$
\text { Epacris }
$$

Det. by:

Date:

Field characters:

Lat:: $384 / 2$ ) Long.:

Alt.:

Habitat:

$$
(3842)
$$

Loc. name(s):

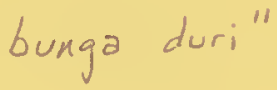

Med. use(s):

Part(s) used:

General notes:

Hazard notes:

Collector(s): MeDonald $\xi$ I smail

$$
\text { No.: } 3843 \text { Date: } 21 \text { Dul } 1993
$$

Sample plant part(s): LF $U 44 z-7833-y$

NCI code number(s): Tw $\quad-7934-z$

Collected under the auspices of:

U.S. National Cancer Institute, Bethesda, MD.

University of Illinois at Chicago, Chicago, IL.

Arnold Arboretum, Harvard University, Cambridge, MA.

Field Museum of Natural History, Chicago, IL.

Host Institution: 


\section{PLANTS OF}

This is a voucher specimen for anticancer testing: YES $\_$NO

Fam.: Myrtaceae

Det. by:

Date:

Field characters:

Lat.: 3842 Long.:

Alt.:

Habitat:

$$
(38 \mathrm{k} / 2)
$$

Loc. name(s):

Med. use(s):

Part(s) used:

General notes:

Hazard notes:

Collector(s): Mc Donald $\xi_{1}$ Ismail

$$
\text { No.: } 3844 \text { Date: } 21 \text { Dul } 1993
$$

Sample plant part(s): $\quad L F \quad \cup 44 Z-7835-A$

NCI code number(s): $\begin{array}{ll}T \omega & -7836-C \\ B A & -7837-F\end{array}$

Collected under the auspices of:

U.S. National Cancer Institute, Bethesda, MD.

University of Illinois at Chicago, Chicago, IL.

Arnold Arboretum, Harvard University, Cambridge, MA.

Field Museum of Natural History, Chicago, IL.

Host Institution: 


\section{PLANTS OF}

This is a voucher specimen for anticancer testing: YES NO

Fam.: Araliaceae

Det. by:

Date:

Field characters:

Lat.: $(35 \%$ Long.:

Alt:

Habitat:

$$
(\geq 8212)
$$

Loc. name(s): "Hohope"

Med. use(s):

Part(s) used:

General notes:

Hazard notes:

Collector(s): McDonald \& Ismail

$$
\text { No.: } 3845 \text { Date: } 21 \text { Dul } 1993
$$

Sample plant part(s): $\angle F \quad U 44 Z-7538-G$

NCI code number(s):

$$
\begin{array}{ll}
P_{E}^{-} & -7539-\mathrm{H} \\
B A & -7840-I
\end{array}
$$

Collected under the auspices of:

U.S. National Cancer Institute, Bethesda, MD.

University of Illinois at Chicago, Chicago, IL.

Arnold Arboretum, Harvard University, Cambridge, MA.

Field Museum of Natural History, Chicago, IL.

Host Institution: $E$ 


\section{PLANTS OF}

This is a voucher specimen for anticancer testing: YES $\angle$ NO

Fam.: Ericaceae

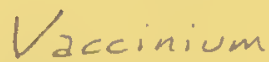

Det. by:

Date:

Field characters:

Lat: $(3842) \quad$ Long.:

Alt :

Habitat: $(3842)$

Loc. name(s):

Med. use(s):

Part(s) used:

General notes:

Hazard notes:

Collector(s): McDonald

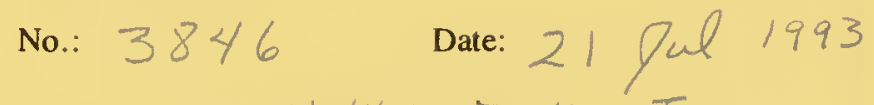

Sample plant part(s): $\quad$ F $U 44 Z-7841-J$

NCI code number(s): TW $\quad-7842-K$

Collected under the auspices of:

U.S. National Cancer Institute, Bethesda, MD.

University of Illinois at Chicago, Chicago, IL.

- Amold Arboretum, Harvard University, Cambridge, MA.

Field Museum of Natural History, Chicago, IL.

Host Institution: $B D, \angle, \cdots$ 


\section{PLANTS OF}

This is a voucher specimen for anticancer testing: YES NO

Fam.: Coprifoliaceae

\section{Viburnum}

Det. by:

Date:

Field characters: (Cow $\ldots$ shive $3-5$ m tall, L, wrle...

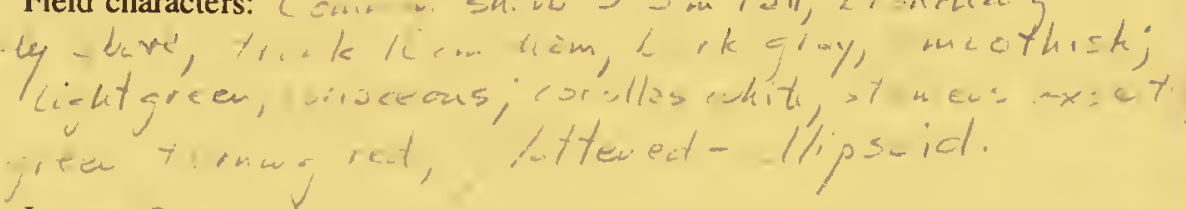

Loc: $\$ 842$ )

Lat.: $(=84 / 2)$ Long.:

Alt :

Habitat:

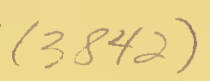

Loc. name(s):

Med. use(s):

Part(s) used:

General notes:

Hazard notes:

Collector(s): Me Dovald \& Isinail

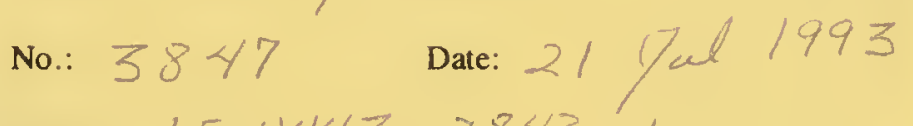

Sample plant part(s): $\quad$ F $\cup 44 Z-7843-\angle$

$\mathrm{NCI}$ code number(s):

$$
\text { Tw }-7844-M
$$

BA

$-7845 \cdot N$

Collected under the auspices of:

U.S. National Cancer Institute, Bethesda, MD.

University of Illinois at Chicago, Chicago, IL.

- Arnold Arboretum, Harvard University, Cambridge, MA.

Field Museum of Natural History, Chicago, IL. 


\section{PLANTS OF}

This is a voucher specimen for anticancer testing: YES $\longleftarrow$ NO

Fam.:

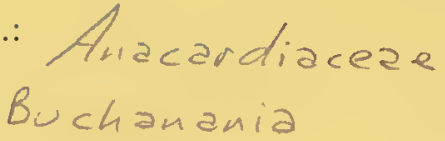

Det. by:

Date:

Field characters: i ceisionst tice /lew toll, l, wh che.

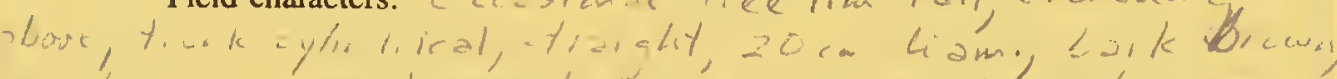
ate. $b-r k, 2 . t=4$; crithes

Loc.:

$$
(3,342)
$$

Lat.: $(3942)$ Long.:

Alt :

Habitat:

$$
(\geq 8</ 2)
$$

Loc. name(s): "Mor on fo""

Med. use(s):

Part(s) used:

General notes:

Hazard notes:

Collector(s): Mc Danald É Ismail

$$
\text { No.: } 3848 \text { Date: } 21 \text { gul } 1993
$$

Sample plant part(s): $\quad \angle F \quad U 44 Z-7846-0$

$\mathrm{NCl}$ code number(s): $\quad B A$

Collected under the auspices of:

$$
\begin{aligned}
& -7847-P \\
& -7849-Q
\end{aligned}
$$

U.S. National Cancer Institute, Bethesda, MD.

the University of Illinois at Chicago, Chicago, IL.

- Arnold Arboretum, Harvard University, Cambridge, MA.

Field Museum of Natural History, Chicago, IL. 


\section{PLANTS OF}

This is a voucher specimen for anticancer testing: YES NO

Fam.: Rhamudceae Aphitonia

Det. by:

Date:

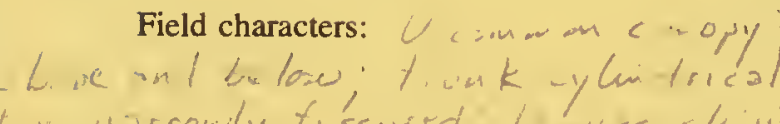

tan, Harrowly fo'ssured, h cues ahiny milper belów

bere; wits abow tant,

Loc: $(3542)$

Lat: $(3842)$ Long.:

Alt :

Habitat: $(3842)$

Loc. name(s):

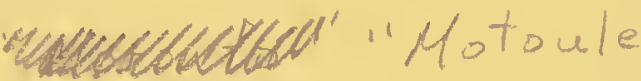

Med. use(s):

Part(s) used:

General notes:

Hazard notes:

Collector(s): Me Dowald E Ismail

$$
\text { No.: } 3849 \text { Date: } 21 \text { low } 1993
$$

Sample plant part(s): $\quad L F \quad U 44 Z-7849-R$

NCI code number(s): TW $\quad-7850-5$

\begin{tabular}{ll}
$F R$ & $-7851-T$ \\
\hline F & $-7852-U$
\end{tabular}

Collected under the auspices of:
U.S. National Cancer Institute, Bethesda, MD.

University of Illinois at Chicago, Chicago, IL.

Arnold Arboretum, Harvard University, Cambridge, MA.

Field Museum of Natural History, Chicago, IL. 


\section{PLANTS OF}

This is a voucher specimen for anticancer testing: YES NO

Fam.: Sterculiaceae stereulia

Det. by:

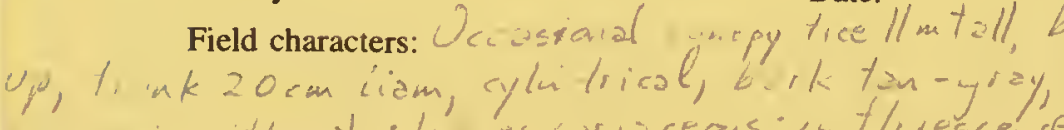

Date:

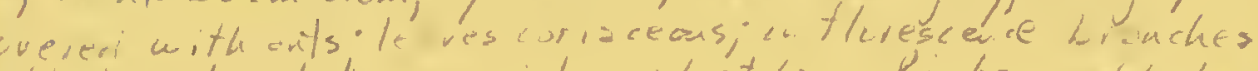

chlish, calyx tube jeenish, ted at base, hanbs
gleen, pobescent.
Loc.:

Loc.: $(3842)$

Lat.: ( 2842 ) Long.:

Alt.:

Habitat: $(3842)$

Loc. name(s):

Med. use(s):

Part(s) used:

General notes:

Hazard notes:

Collector(s): Me Dorald \& I smail

$$
\text { No: } 3850 \text { Date: } 22 \text { \%ol } 1993
$$

Sample plant part(s): $\angle F \quad U 44 Z-7853-V$

NCI code number(s):

$$
\text { Tw }
$$

BA

$-7354-w$

$-7855-x$

Collected under the auspices of:

U.S. National Cancer Institute, Bethesda, MD.

University of Illinois at Chicago, Chicago, IL.

Arnold Arboretum, Harvard University, Cambridge, MA.

Field Museum of Natural History, Chicago, IL. 


\section{PLANTS OF}

This is a voucher specimen for anticancer testing: YES $\_$NO

Fam.: Burseraceae

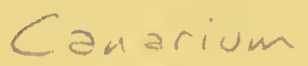

Det. by:

Date:

Field characters: Ceminon canopy yiee $15 \mathrm{~m}$ tall, ti an lo

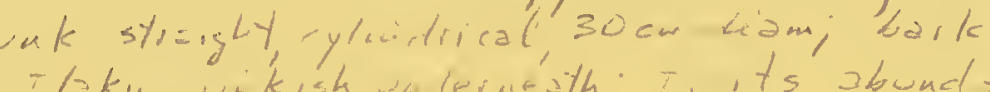

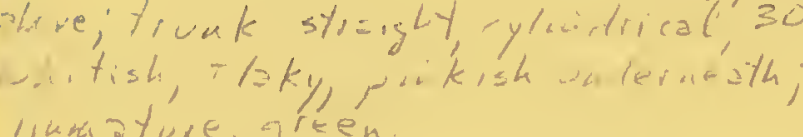
like ature, green

Loc: $(3842)$

Lat:: $(3,342)$ Long.:

Alt.:

Habitat:

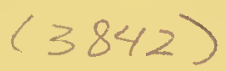

Loc. name(s): "Bendor,"

Med. use(s):

Part(s) used:

General notes:

Hazard notes:

Collector(s): Mc Donald E Ismail

$$
\text { No.: } 3851 \quad \text { Date: } 22
$$

Sample plant part(s): $\frac{\angle F}{T \omega}$ U4 $Z Z-7856-Y$

NCI code number(s):

Collected under the auspices of:

$$
\begin{aligned}
& \text { QA } \\
& \text { F R } R \text { : }
\end{aligned}
$$$$
-7857-z
$$

U.S. National Cancer Institute, Bethesda, MD.

University of Illinois at Chicago, Chicago, IL.

- Arnold Arboretum, Harvard University, Cambridge, MA. Field Museum of Natural History, Chicago, IL. 


\section{PLANTS OF}

This is a voucher specimen for anticancer testing: YES $\longleftarrow \mathrm{NO}$

Fam.: Arerrhoaceae

$$
\text { Sarcotheca }
$$

Det. by:

Date:

Field characters: ceasianal , est-moigin tree $8 \mathrm{~m}$

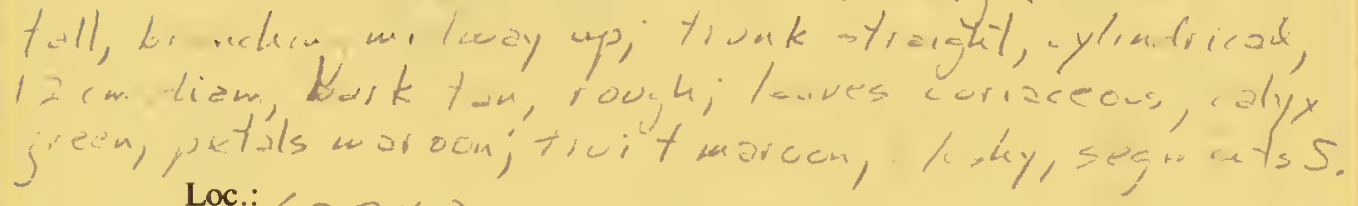

Loc.: $(3842)$

Lat.: $(=842)$ Long.: Alt.:

Habitat: $(3842)$

Loc. name(s): "Benono"

Med. use(s):

Part(s) used: fruit edible, sour

General notes:

Hazard notes:

Collector(s): McDonald \& Ismail

$$
\text { No.: } 3852 \text { Date: } 22 \text { gul } 1993
$$

Sample plant part(s): LF U44Z-7860 - F

NCI code number(s):

$$
\text { Thtw }
$$

$$
B A \cup 44 Z-7861-G
$$

Collected under the auspices of:

U.S. National Cancer Institute, Bethesda, MD.

University of Illinois at Chicago, Chicago, IL.

- Arnold Arboretum, Harvard University, Cambridge, MA.

Field Museum of Natural History, Chicago, IL. 


\section{PLANTS OF}

This is a voucher specimen for anticancer testing: YES NO

Fam.: Rosaceae Prunus

Det. by:

Field characters:

Date:

Occosiand Yic $12 \mathrm{~m} / \mathrm{all}$, $\%$, nk trical, $25 \mathrm{~cm}$ liam,

Loc: $(3842)$
Lent green.

Lat.: $(3842)$ Long.:

Alt: :

Habitat: $(3842)$

Loc. name(s):

Med. use(s):

Part(s) used:

General notes:

Hazard notes:

Collector(s): McDonald \&' Ismail

$$
\text { No: } 3853 \text { Date: } 22 \text { fal } 1993
$$

Sample plant part(s): $\quad$ F $U 44 Z-7962-H$

$\mathrm{NCI}$ code number(s):

$$
\begin{array}{ll}
\text { Tw } & -7863-I \\
B A & -7864-J
\end{array}
$$

Collected under the auspices of:

U.S. National Cancer Institute, Bethesda, MD.

University of Illinois at Chicago, Chicago, IL.

- Arnold Arboretum, Harvard University, Cambridge, MA.

Field Museum of Natural History, Chicago, IL. 


\section{PLANTS OF}

This is a voucher specimen for anticancer testing: YES $\swarrow$ NO

Fam.: Myrtaceae

Metrosideros

Det. by:

Date:

Field characters:

Loc.: $(3,84 / 2$ )

Lat.: $(3842)$ Long.:

Alt.:

Habitat:

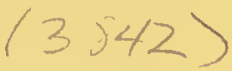

Loc. name(s): "Nona"

Med. use(s):

Part(s) used:

General notes:

Hazard notes:

Collector(s): Mc Donald \&' Ismail

$$
\text { No.: } 3854 \text { Date: } 22 \text { Peal } 1993
$$

Sample plant part(s): LF $U 4 / Z-7365-K$

$\mathrm{NCI}$ code number(s):

$$
\begin{array}{ll}
\pi \omega & -7866-\angle \\
B A & -7867-M
\end{array}
$$

Collected under the auspices of:

U.S. National Cancer Institute, Bethesda, MD.

University of Illinois at Chicago, Chicago, IL.

Arnold Arboretum, Harvard University, Cambridge, MA.

Field Museum of Natural History, Chicago, IL. 


\section{PLANTS OF}

This is a voucher specimen for anticancer testing: YES _ $\_$NO

Fam.: Proteaceae

Grevillea

Det. by:

Date:

Field characters:

Lat.: $(3842)$ Long.:

Alt :

Habitat: $(3 \delta 42)$

Loc. name(s): "Keulan tangkeno"

Med. use(s):

Part(s) used:

General notes:

Hazard notes:

Collector(s): McDonald \& Ismail

$$
\text { No.: } 3855 \text { Date: } 22 \text { Jul } 1993
$$

Sample plant part(s): $\begin{array}{rr}L F & \cup 44 Z-7868-N \\ & \text { Tu) }\end{array}$

NCI code number(s): $B A \quad-7870-P$

Collected under the auspices of:

FR

U.S. National Cancer Institute, Bethesda, MD.

University of Illinois at Chicago, Chicago, IL.

Arnold Arboretum, Harvard University, Cambridge, MA.

Field Museum of Natural History, Chicago, IL. 


\section{PLANTS OF}

This is a voucher specimen for anticancer testing: YES NO

Fam.: Casuarinaceae

\section{Casuarina}

Det. by:

Field characters:

Loc.: $(5842)$

Lat.: $=842$ ) Long.:

Date:

Field characters.

Habitat: 3942$)$

Alt.:

Loc. name(s): "Vai"

Med. use(s):

Part(s) used:

General notes:

Hazard notes:

Collector(s): Mc Danald हो Ismail

$$
\text { No.: } 3856 \text { Date: } 22 \text { Tul } 1993
$$

Sample plant part(s): LF $U 4 / Z-7872-R$

NCI code number(s): $B A$

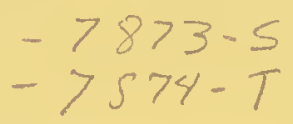

Collected under the auspices of:

U.S. National Cancer Institute, Bethesda, MD.

University of Illinois at Chicago, Chicago, IL.

- Arnold Arboretum, Harvard University, Cambridge, MA.

Field Museum of Natural History, Chicago, IL. 


\section{PLANTS OF}

This is a voucher specimen for anticancer testing: YES $\_$NO

Fam.: Apocyuaceae

Det. by:

Date:

Field characters:

$15 \mathrm{~m}+\mathrm{all}$,

ipitruk straght, ykidoical,

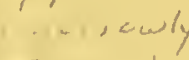

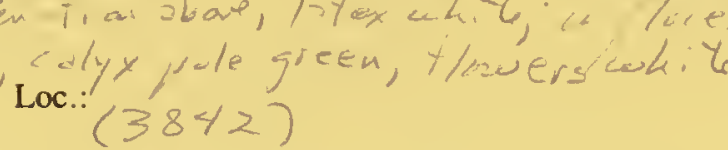

Lat.: $(3 \delta / 2)$ Long.:

Alt:

Habitat: $(3842)$

Loc. name(s):

Med. use(s):

Part(s) used:

General notes:

Hazard notes:

Collector(s): $M<$ Douald $\xi$ /smail

$$
\text { No.: } 5857 \text { Date: } 22 \mathrm{Pal} / 993
$$

Sample plant part(s): $\angle F \quad U / 4 Z Z-7875-U$

NCI code number(s): $\begin{array}{ll}\text { Tw } & -7876-\mathrm{V} \\ B A & -7877-\mathrm{W}\end{array}$

Collected under the auspices of:

U.S. National Cancer Institute, Bethesda, MD.

University of Illinois at Chicago, Chicago, IL.

- Arnold Arboretum, Harvard University, Cambridge, MA.

Field Museum of Natural History, Chicago, IL. 


\section{PLANTS OF}

Sulauresi

This is a voucher specimen for anticancer testing: YES $\_$NO

Fam.: Araliaceae

Det. by:

Date:

Field characters:

$$
(3542)
$$

Lat.:( 3842$)$ Long.:

Alt.:

Habitat: $(3842)$

Loc. name(s): "Banki

Med. use(s):

Part(s) used:

General notes:

Hazard notes:

Collector(s):

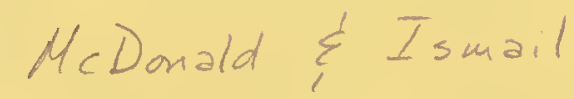

$$
\text { No.: } 3858 \quad \text { Date: } 22 \mathrm{Jul} / 993
$$

Sample plant part(s): LF $U 44 z-7878-x$

NCI code number(s): $\quad B A$

Collected under the auspices of:

$$
\begin{array}{ll}
B A & -7880-Z \\
\text { INEL } & -7881-A
\end{array}
$$

U.S. National Cancer Institute, Bethesda, MD.

University of Illinois at Chicago, Chicago, IL.

- Arnold Arboretum, Harvard University, Cambridge, MA.

Field Museum of Natural History, Chicago, IL. 


\section{PLANTS OF}

This is a voucher specimen for anticancer testing: YES $\_$NO

Fam.: Myrtaceae

$$
\text { Eugenia }
$$

Det. by:

Date:

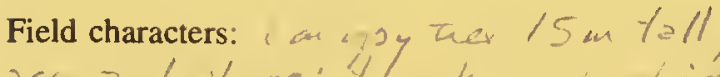

Lat.: $(\geqslant 842)$ Long.:

Alt.:

Habitat: $(3842)$

Loc. name(s): "Dambukeo"

Med. use(s):

Part(s) used:

General notes:

Hazard notes:

Collector(s): Mc Denald \&́ Ismail

$$
\text { No: } 3859 \quad \text { Date: } 22 \mathrm{Dul} / 993
$$

Sample plant part(s): $\angle F \quad U 44 Z-7882-C$

$\mathrm{NCl}$ code number(s):

Collected under the auspices of:

U.S. National Cancer Institute, Bethesda, MD.

University of Illinois at Chicago, Chicago, IL.

- Amold Arboretum, Harvard University, Cambridge, MA.

Field Museum of Natural History, Chicago, IL. 


\section{PLANTS OF}

This is a voucher specimen for anticancer testing: YES NO

Fam.: Tiliceeae

Det. by:

Date:

Field characters:

Loc.: $(3842)$

Lat. $(3542$ ) Long.:

Alt :

Habitat: $(3842)$

Loc. name(s):

Med. use(s):

Part(s) used:

General notes:

Hazard notes:

Collector(s): He Donald E Ismail

$$
\text { No.: } 3860 \text { Date: } 230 \mathrm{ful} / 993
$$

Sample plant part(s): $\angle F \quad \cup 44 Z-7885-H$

NCI code number(s): $\begin{array}{ll}T \omega & -7886-I \\ B A & -7887-J\end{array}$

Collected under the auspices of:

U.S. National Cancer Institute, Bethesda, MD.

University of Illinois at Chicago, Chicago, IL.

- Arnold Arboretum, Harvard University, Cambridge, MA.

Field Museum of Natural History, Chicago, IL. 


\section{PLANTS OF}

This is a voucher specimen for anticancer testing: YES NO

Fam.: ? Celastraceae

Det. by:

Date:

Field characters:

Lat: $(35 \% 2)$ Long.:

Alt :

Habitat: $(\geq 542)$

Loc. name(s):

Med. use(s):

Part(s) used:

General notes:

Hazard notes:

Collector(s): Mc Donald $\sum$ Ismail

$$
\text { No.: } 3861 \text { Date: } 23 / \text { ul } / 993
$$

Sample plant part(s): LF $144 Z-7589-K$

$$
\text { NCI code number(s): } \begin{array}{ll}
\text { Tw } & -7889-L \\
B A & -7890-M
\end{array}
$$

Collected under the auspices of:

U.S. National Cancer Institute, Bethesda, MD.

University of Illinois at Chicago, Chicago, IL.

- Arnold Arboretum, Harvard University, Cambridge, MA.

Field Museum of Natural History, Chicago, IL. 


\section{PLANTS OF}

This is a voucher specimen for anticancer testing: YES NO

Fam.: Protecceae

Helicia

Det. by:

Date:

Field characters:

Loc: t tuse

$$
(3842)
$$

Lat.: $(3542)$ Long.:

Alt.:

Habitat: $(2842)$

Loc. name(s):

Med. use(s):

Part(s) used:

General notes:

Hazard notes:

Collector(s): HC Danald \& Ismail

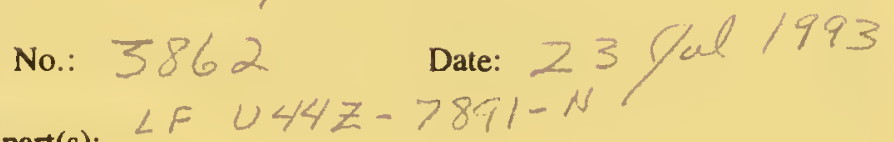

Sample plant part(s): $\angle F \quad \cup 44 Z-7891-N$

NCI code number(s): $B A$

$-7892-0$

$F R$

Collected under the auspices of:

$-7893-P$

U.S. National Cancer Institute, Bethesda, MD.

University of Illinois at Chicago, Chicago, $\mathrm{IL}$.

- Arnold Arboretum, Harvard University, Cambridge, MA.

Field Museum of Natural History, Chicago, IL. 


\section{PLANTS OF}

This is a voucher specimen for anticancer testing: YES $\mathrm{NO}$

Fam.: Celastraceae

Det. by:

Date:

Field characters: tinath

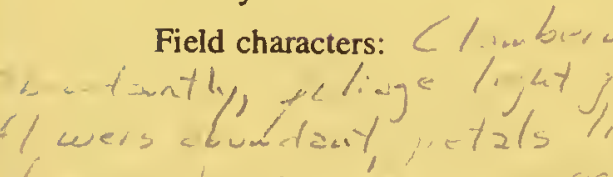

- wers aluatawy

$4=i c$ l.

Loc:

$$
(382 / 2)
$$

Lat.: $(3 \delta 42)$ Long.:

Alt.:

Habitat: $(3842)$

Loc. name(s):

Med. use(s):

Part(s) used:

General notes:

Hazard notes:

Collector(s): He Donald \& Ismail

$$
\text { No.: } 3863 \quad \text { Date: } 23 \mathrm{Jul} / 993
$$

Sample plant part(s): LF U44Z-7875-R

NCI code number(s): Tw $\quad-7896-5$

Collected under the auspices of:

U.S. National Cancer Institute, Bethesda, MD.

University of Illinois at Chicago, Chicago, IL.

- Arnold Arboretum, Harvard University, Cambridge, MA.

Field Museum of Natural History, Chicago, IL. 


\section{PLANTS OF}

This is a voucher specimen for anticancer testing: YES NO

Fam.: Wite aceae

$$
\text { Callicarpa }
$$

Det. by:

Date:

Field characters:

Loc.: atio vi,<smiles></smiles>

Lat.: $(\geqslant \$ 4 / 2)$ Long.:

Alt :

Habitat: $(35 / 2)$

Loc. name(s):

Med. use(s):

Part(s) used:

General notes:

Hazard notes:

Collector(s):

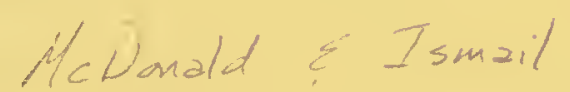

$$
\text { No.: } 3864 \text { Date: } 23 \text { / ut } 1793
$$

Sample plant part(s): $\quad \angle F \cup 44 Z-7897-T$

$\mathrm{NCl}$ code number(s):

$$
\text { Tw }
$$

$B, A$

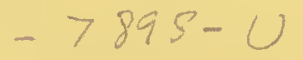

Collected under the auspices of:

U.S. National Cancer Institute, Bethesda, MD.

University of Illinois at Chicago, Chicago, IL.

- Arnold Arboretum, Harvard University, Cambridge, MA. Field Museum of Natural History, Chicago, IL. 


\section{PLANTS OF}

This is a voucher specimen for anticancer testing: YES $\longleftarrow$ NO

Fam.: Apocyuaceae ? Alyxia

Det. by:

Date:

Field characters: L Mmbeing liana to $10 \mathrm{~m}$ high; stans

Lat.: $(\geq 842)$ Long.: Alt.:

Habitat: $(2842)$

Loc. name(s):

Med. use(s):

Part(s) used:

General notes:

Hazard notes: [Sop can blind]

Collector(s): McDonald E' Isuail

$$
\text { No.: } 3865 \text { Date: } 23 / \mathrm{Jul} / 993
$$

Sample plant part(s): LF U44Z-7900-W

NCI code number(s):

$$
\text { Tw } \quad-7901-x
$$

Collected under the auspices of:

U.S. National Cancer Institute, Bethesda, MD.

University of Illinois at Chicago, Chicago, IL.

- Arnold Arboretum, Harvard University, Cambridge, MA.

Field Museum of Natural History, Chicago, IL. 


\section{PLANTS OF}

This is a voucher specimen for anticancer testing: YES NO

Fam.: ? Rhamuaceae

Det. by:

Date:

Field characters:

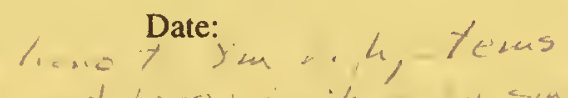

ites hous

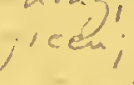

it car ature,

$+t h$

l

Loc: $(\geq 84 / 2)$

Lat:: $(38 \%$ Long.:

Alt :

Habitat: $(38 \% 2)$

Loc. name(s):

Med. use(s):

Part(s) used:

General notes:

Hazard notes:

Collector(s): Hc Danald \& I smail

$$
\text { No.: } 3866 \text { Date: } 23 \mathrm{ful} / 793
$$

Sample plant part(s): $\angle F \quad U 44 Z-7902-Y$

$\mathrm{NCl}$ code number(s):

$$
\text { Tw }-7903-z
$$

Collected under the auspices of:

U.S. National Cancer Institute, Bethesda, MD.

University of Illinois at Chicago, Chicago, IL.

- Arnold Arboretum, Harvard University, Cambridge, MA.

Field Museum of Natural History, Chicago, IL. 


\section{PLANTS OF}

This is a voucher specimen for anticancer testing: YES NO

Fam.: Myristicaceae Hors fie lia

Det. by:

Date:

Field characters:

Lat.: $(3842)$ Long.:

Alt :

Habitat:<smiles>[CH]C=[As]=C1CCCC1</smiles>

Loc. name(s):

Med. use(s):

Part(s) used:

General notes:

Hazard notes:

Collector(s): $M<D$ onald $\xi$ Ismail

$$
\text { No.: } 3867 \text { Date: } 231 \mathrm{fal} / 993
$$

Sample plant part(s): $\angle F \cup 44 Z-7904-A^{\prime}$

NCI code number(s):

$$
\text { Tw }
$$

$B A$

$-7905-C$

$-7906-F$

Collected under the auspices of:

U.S. National Cancer Institute, Bethesda, MD.

University of Illinois at Chicago, Chicago, IL.

- Amold Arboretum, Harvard University, Cambridge, MA.

Field Museum of Natural History, Chicago, IL. 


\section{PLANTS OF}

This is a voucher specimen for anticancer testing: YES $\longleftarrow$ NO

Fam.: Myrsinactae Mavea

Det. by:

Date:

Field characters:

Loc: $(2542)$

Lat.: $(38 \% 2)$ Long.:

Alt :

Habitat: $(3842)$

Loc. name(s):

Med. use(s):

Part(s) used:

General notes:

Hazard notes:

Collector(s): H/C Donald \& Ismail

$$
\text { No.: } 3868 \text { Date: } 23 \text { gul } 1993
$$

Sample plant part(s): $\angle F \quad \cup 44 Z-7907-G$

$\mathrm{NCl}$ code number(s):

Collected under the auspices of:

U.S. National Cancer Institute, Bethesda, MD.

University of Illinois at Chicago, Chicago, IL.

- Arnold Arboretum, Harvard University, Cambridge, MA.

Field Museum of Natural History, Chicago, IL. 


\section{PLANTS OF}

This is a voucher specimen for anticancer testing: YES NO

Fam.: Him os aceae

? Albizia

Det. by:

Date:

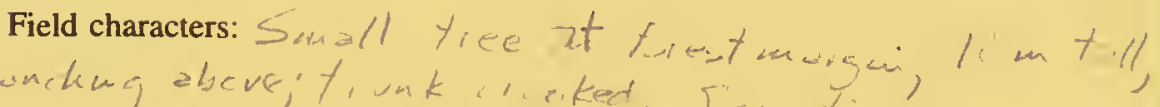

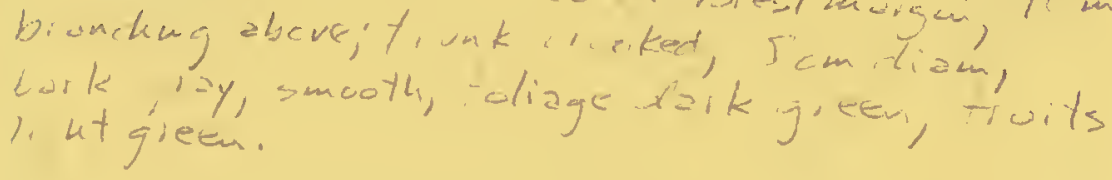

Loc: $(38 \% 2)$

Lat: $(3<42)$ Long.:

Alt.:

Habitat: $\geq 3 / 2$ )

Loc. name(s):

Med. use(s):

Part(s) used:

General notes:

Hazard notes:

Collector(s): HeDenald $\xi$ Ismail

$$
\text { No.: } 3869 \quad \text { Date: } 23 / 7 \text { ul } 1993
$$

Sample plant part(s): $\angle F+F K$ U) $44 Z-7909-I$

NCI code number(s):

$$
\text { TW }
$$

Collected under the auspices of:

U.S. National Cancer Institute, Bethesda, MD.

University of Illinois at Chicago, Chicago, IL.

- Arnold Arboretum, Harvard University, Cambridge, MA.

Field Museum of Natural History, Chicago, IL. 


\section{PLANTS OF}

This is a voucher specimen for anticancer testing: YES NO

Fam.: Euphorbiaceae Glochidion

Det. by:

Date:

Field characters:

Loc.:

1,1,

ic

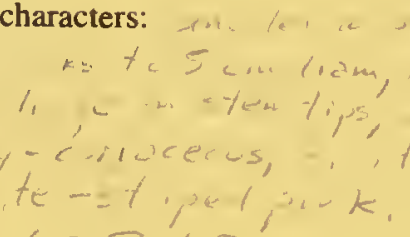

$(2842)$

Lat.: $/ \geq 3 \% 2$ Long.:

Alt :

Habitat:

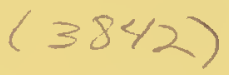

Loc. name(s):

Med. use(s):

Part(s) used:

General notes:

Hazard notes:

Collector(s): $/ / C$ Donald \& Ismail

$$
\text { No: } 3870 \quad \text { Date: } 24 / \mathrm{cal} / 993
$$

Sample plant part(s): $\angle F \cup 44 Z-7912-\angle$

NCI code number(s):
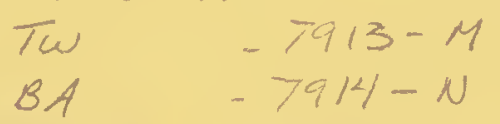

Collected under the auspices of:

U.S. National Cancer Institute, Bethesda, MD.

University of Illinois at Chicago, Chicago, IL.

- Amold Arboretum, Harvard University, Cambridge, MA.

Field Museum of Natural History, Chicago, IL. 


\section{PLANTS OF}

This is a voucher specimen for anticancer testing: YES NO

Fam.: Myrtaceae

Det. by:

Date:

Field characters:

Loc.: $(2342)$

Lat.: $(38<2)$ Long:

Alt :

Habitat: $(3842)$

Loc. name(s):

Med. use(s):

Part(s) used:

General notes:

Hazard notes:

Collector(s):

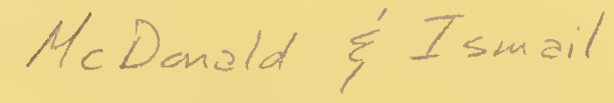

No.: 387

Date: 24 Pul /993

Sample plant part(s): $\angle F \quad U 44 Z-7715-0$

$\mathrm{NCl}$ code number(s):

Collected under the auspices of:

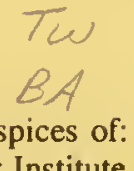

U.S. National Cancer Institute, Bethesda, MD.

University of Illinois at Chicago, Chicago, IL.

- Arnold Arboretum, Harvard University, Cambridge, MA. Field Museum of Natural History, Chicago, IL. 


\section{PLANTS OF}

This is a voucher specimen for anticancer testing: YES NO

Fam.: Hyrtaceae

Det. by:

Date:

Field characters:

Loc.: $(334 / 2)$

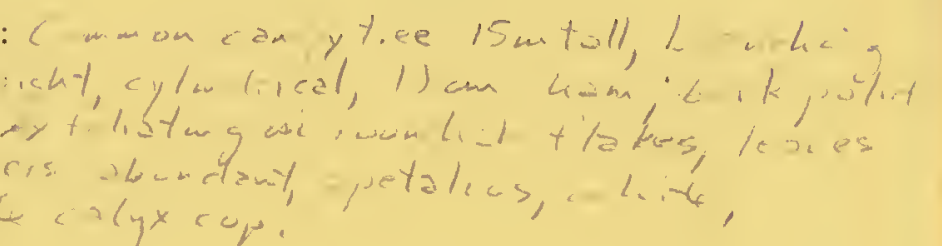

Lat.: ( 3842$)$ Long.:

Alt: :

Habitat:

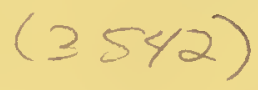

Loc. name(s):

Med. use(s):

Part(s) used:

General notes:

Hazard notes:

Collector(s): McDonald \& Ismail

$$
\text { No.: } 3872 \text { Date: } 24 \text { Pul } / 993
$$

Sample plant part(s): LF U44Z- $\quad$ G $/ 5-R$

NCI code number(s):

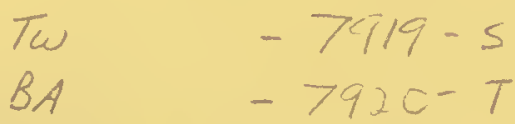

Collected under the auspices of:

U.S. National Cancer Institute, Bethesda, MD.

University of Illinois at Chicago, Chicago, IL.

- Arnold Arboretum, Harvard University, Cambridge, MA.

Field Museum of Natural History, Chicago, IL. 


\section{PLANTS OF}

This is a voucher specimen for anticancer testing: YES NO

Fam.: Myristicaceae

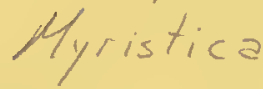

Det. by:

Date:

Field characters:

Loc.:

$$
(38 \% 2)
$$

Lat.: $(\geq \rho \% 2)$ Long.:

Alt :

Habitat: 3842$)$

Loc. name(s):

Med. use(s):

Part(s) used:

General notes:

Hazard notes:

Collector(s): $4<D$ anald है Ismail

No: 5873 Date: $24 \mathrm{gul} / 943$

Sample plant part(s): $\operatorname{LF}_{T \omega} \begin{array}{r}W 4 Z-7721-U \\ -7922-V\end{array}$

NCI code number(s): $B A$

$-7923-W$

Collected under the auspices of:

U.S. National Cancer Institute, Bethesda, MD.

University of Illinois at Chicago, Chicago, IL.

- Arnold Arboretum, Harvard University, Cambridge, MA.

Field Museum of Natural History, Chicago, IL. 


\section{PLANTS OF}

This is a voucher specimen for anticancer testing: YES $\_$NO

Fam.: Magnoliaceae

$$
\text { ? Talauma }
$$

Det. by:

Date:

Field characters:

Loc.:

$$
(3342)
$$

Lat.: $<3342$ ) Long.:

Alt.:

Habitat: $(38 \% 2)$

Loc. name(s):

Med. use(s):

Part(s) used:

General notes:

Hazard notes:

Collector(s): MCD onald 乡' Ismail

$$
\text { No.: } 3874 \quad \text { Date: } 24 \text { /al } / 993
$$

Sample plant part(s): $\angle F \quad U / 4 Z=7724-X$

$\mathrm{NCI}$ code number(s):

$$
\text { Tw }
$$

$B A$

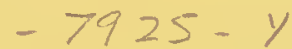

$-7926-z$

Collected under the auspices of:

U.S. National Cancer Institute, Bethesda, MD.

University of Illinois at Chicago, Chicago, IL.

Arnold Arboretum, Harvard University, Cambridge, MA.

Field Museum of Natural History, Chicago, IL.

Host Institution: $\mathrm{B} C<\angle 1 \mathrm{~N}$ 


\section{PLANTS OF}

This is a voucher specimen for anticancer testing: YES NO

Fam.: Aqui foliaceae

\section{Ilex}

Det. by:

Date:

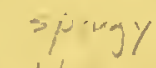

l.

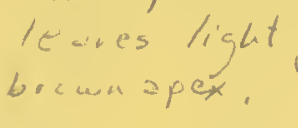

Loc.:

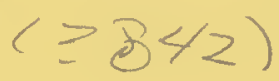

Lat.: $(3842)$ Long.:

Alt:

Habitat: $(3842)$

Loc. name(s):

Med. use(s):

Part(s) used:

General notes:

Hazard notes:

Collector(s): MCDanald \& Ismail

$$
\text { No.: } 3875 \text { Date: } 24 \text { Jul /993 }
$$

Sample plant part(s): $\angle F \quad U 44 Z-7927-A$

NCI code number(s):

$$
\begin{array}{ll}
\text { TW } & -7928-C \\
B A & -7929-F
\end{array}
$$

Collected under the auspices of:

U.S. National Cancer Institute, Bethesda, MD.

University of Illinois at Chicago, Chicago, IL.

- Arnold Arboretum, Harvard University, Cambridge, MA.

Field Museum of Natural History, Chicago, IL.

Host Institution: $B O$ 


\section{PLANTS OF}

This is a voucher specimen for anticancer testing: YES $\mathrm{NO}$

Fam.: Calastraceae

$$
\text { Evonymus }
$$

Det. by:

Date:

Field characters:

Loc: $(342)$

Lat.: $(38 / 2)$ Long.:

Alt.:

Habitat: $(3842)$

Loc. name(s):

Med. use(s):

Part(s) used:

General notes:

Hazard notes:

Collector(s):

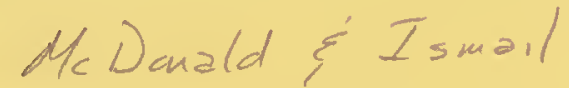

$$
\text { No.: } 3876 \quad \text { Date: } 24 \text { / al } 1993
$$

Sample plant part(s): $\angle F \quad U 4 / Z-7930-6$

$\mathrm{NCI}$ code number(s):

$$
\text { Tw }
$$$$
-7931-4
$$$$
-7932 \text { I }
$$

Collected under the auspices of:

U.S. National Cancer Institute, Bethesda, MD.

University of Illinois at Chicago, Chicago, IL.

Arnold Arboretum, Harvard University, Cambridge, MA.

Field Museum of Natural History, Chicago, IL.

Host Institution: 


\section{PLANTS OF}

This is a voucher specimen for

anticancer testing: YES NO

Fam.: Celastrocece

Det. by:

Date:

Field characters:

Loc.:

trat 4 t cylow

Lat.: $(384 / 2)$ Long.:

Alt.:

Habitat:

$3842)$

Loc. name(s):

Med. use(s):

Part(s) used:

General notes:

Hazard notes:

Collector(s):

HC Donald F Ismail

$$
\text { No.: } 3877 \text { Date: } 24 \mathrm{fal} / 993
$$

Sample plant part(s): $\quad \angle F \quad \cup 44 z-7933-J$

$\begin{array}{lll} & \text { NCI code number(s): } & -7934-K \\ B A & -7935-L\end{array}$

Collected under the auspices of:

U.S. National Cancer Institute, Bethesda, MD.

University of Illinois at Chicago, Chicago, IL.

Amold Arboretum, Harvard University, Cambridge, MA.

Field Museum of Natural History, Chicago, IL.

Host Institution: 


\section{PLANTS OF}

This is a voucher specimen for anticancer testing: YES $\mathrm{NO}$

Fam.: Theaceae

Alivandra

Det. by:

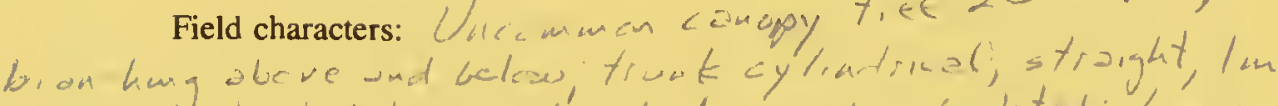

Date: 20 in $t a / 1$,

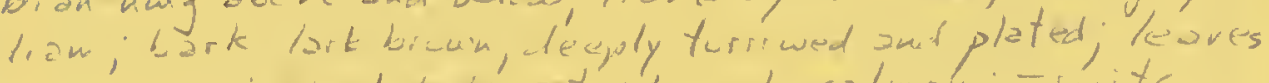
curiacecus, thal buls rotund, pale salmon; i wits

Loc.: $(3842)$

Lat.: $(38 \% 2)$ Long.:

Alt :

Habitat:

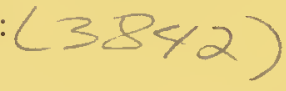

Loc. name(s):

Med. use(s):

Part(s) used:

General notes:

Hazard notes:

Collector(s): HCD anald \& Ismail

$$
\text { No.: } 3878 \quad \text { Date: } 24 / 7 \text { al } / 993
$$

Sample plant part(s): $\quad \angle F \quad \cup 44 Z-7936-M$

$\mathrm{NCl}$ code number(s):

$$
\begin{aligned}
& \text { Tw } \quad-7937-N \\
& \text { BA }-7938-0
\end{aligned}
$$

Collected under the auspices of:

U.S. National Cancer Institute, Bethesda, MD.

University of Illinois at Chicago, Chicago, IL.

- Amold Arboretum, Harvard University, Cambridge, MA.

Field Museum of Natural History, Chicago, IL.

Host Institution: $\mathrm{BO}$ 


\section{PLANTS OF}

This is a voucher specimen for

anticancer testing: YES NO

Fam.: Arecaceae

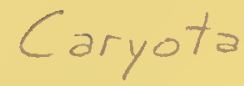

Det. by:

Date:

Field characters:

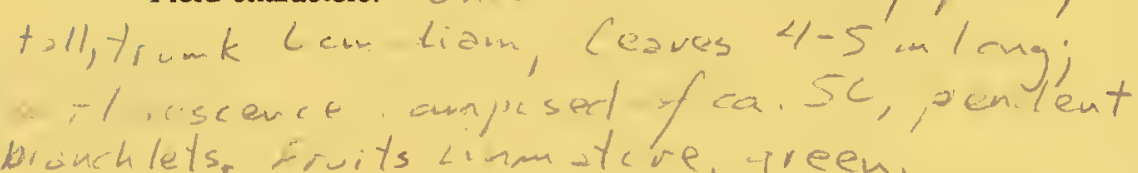

Loc.:

$(3842)$

Lat: $(38 \% 2)$ Long.:

Alt.:

Habitat: $(3342)$

Loc. name(s):

Med. use(s):

Part(s) used:

General notes:

Hazard notes:

Collector(s): McDonald $\xi$ / smail

$$
\text { No.: } 3879 \quad \text { Date: } 24 \mathrm{Ful} / 993
$$

Sample plant part(s): $\quad \angle F \quad \cup 44 Z-7939-P$

NCI code number(s):

Collected under the auspices of:

U.S. National Cancer Institute, Bethesda, MD.

University of Illinois at Chicago, Chicago, IL.

Amold Arboretum, Harvard University, Cambridge, MA.

Field Museum of Natural History, Chicago, IL.

Host Institution: 


\section{PLANTS OF}

This is a voucher specimen for anticancer testing: YES $\measuredangle$ NO

Fam.: Rutaceae

\section{Evodia}

Det. by:

Date:

Field characters: Timberline, cancpy tree, 12 m t-ll, branchen a abure, trunk straigly, aylindrical, $12 \mathrm{~cm} \mathrm{liam;} \mathrm{Lark} \mathrm{light} \mathrm{gray,}$ rough; Lranchargsparingly. foliage lussy-jeen, leaves helt oreen, petals 4 white curiaceolus stamensopposite vetals Loc: $f_{1}$ lament white, anthersex serted, yellow, ovary
y-lobed, green, stylkwhite.

$$
(3842)
$$

Lat:: $(38 \% / 2)$ Long.:

Alt.:

Habitat:

$$
\text { (3842) Uyper timberline }
$$

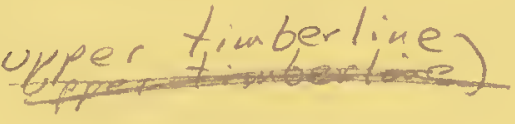

Loc. name(s):

Med. use(s):

Part(s) used:

General notes:

Hazard notes:

Collector(s): HeDanald \& Ismail

$$
\text { No.: } 3881 \text { Date: } 25 \text { D/al } 1993
$$

Sample plant part(s): $\angle F$

$$
\begin{aligned}
U 44 Z & -7940-Q \\
& -7941-R \\
& -7942-S
\end{aligned}
$$

NCI code number(s): ${ }^{T} \mathrm{~A}$

Collected under the auspices of:

U.S. National Cancer Institute, Bethesda, MD.

University of Illinois at Chicago, Chicago, IL. Arnold Arboretum, Harvard University, Cambridge, MA.

Field Museum of Natural History, Chicago, IL. 


\section{PLANTS OF}

This is a voucher specimen for anticancer testing: YES NO

Fam.: L/aeocarpaceae Flaeocarpus

Det. by:

Date:

Field characters: Shoft tree $4 \mathrm{me}$ tall, bianching midcuey

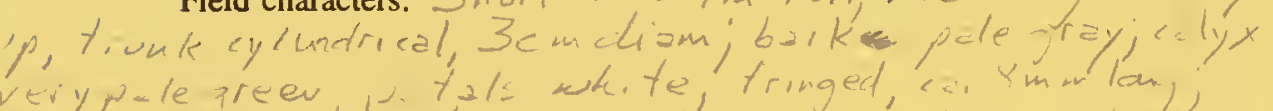
stamens a gueroo's, with swall yellow-laxts at base ylu)

5lamens a nerous, whth sm all yellow
Loc.:
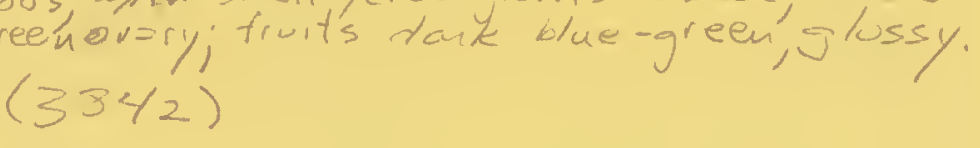

Lat.: $(3842)$ Long.:

Alt: :

Habitat: ( 3542 , wper timberline)

Loc. name(s):

Med. use(s):

Part(s) used:

General notes:

Hazard notes:

Collector(s):

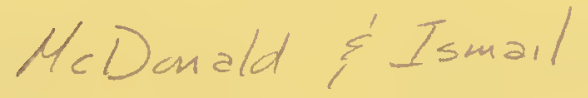

No.: 3882 Date: $25 \%$ al /993

Sample plant part(s): $\quad \angle F \cup 4 / 2-7743-T$

$\mathrm{NCI}$ code number(s):

Collected under the auspices of:

U.S. National Cancer Institute, Bethesda, MD.

University of Illinois at Chicago, Chicago, IL.

Arnold Arboretum, Harvard University, Cambridge, MA.

Field Museum of Natural History, Chicago, IL.

Host Institution: 


\section{PLANTS OF}

This is a voucher specimen for anticancer testing: YES $\_$NO

Fam.: Oleaceae

Ligustrum

Det. by:

Date:

Field characters:

ore and at base, Lasar ctams

latishbrownwi th tan lenticels

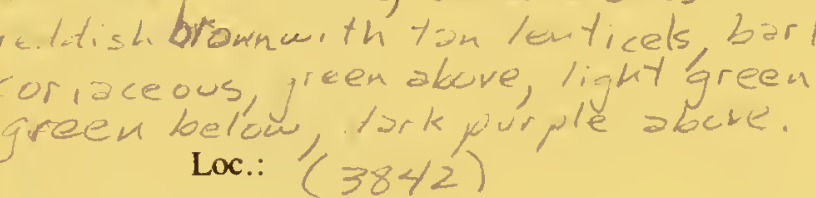
green below, tark pur ple abote.
Loc: $(3842)$

$2-3 m$ tall, bicuchurg

Lat.: $(384 / 2)$ Long.:

Alt.:

Habitat:

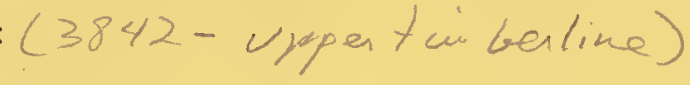

Loc. name(s):

Med. use(s):

Part(s) used:

General notes:

Hazard notes:

Collector(s):

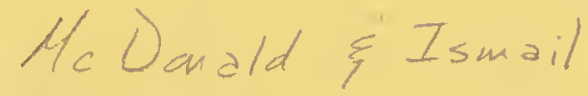

$$
\text { No.: } 3883 \quad \text { Date: } 25 \mathrm{ful} / 993
$$

Sample plant part(s):

NCI code number(s):

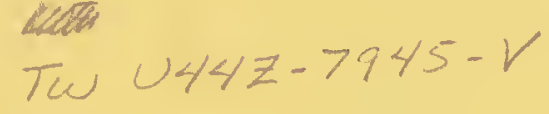

Collected under the auspices of:

U.S. National Cancer Institute, Bethesda, MD.

University of Illinois at Chicago, Chicago, IL.

Amold Arboretum, Harvard University, Cambridge, MA.

Field Museum of Natural History, Chicago, IL.

Host Institution: 
PLANTS OF

This is a voucher specimen for anticancer testing: YES

NO

Fam.: Proteaceae

Macadamia

Bet. by:

Date:

Field characters: (anopiy tree $14 \mathrm{~m} / \mathrm{m} / 1$, branching

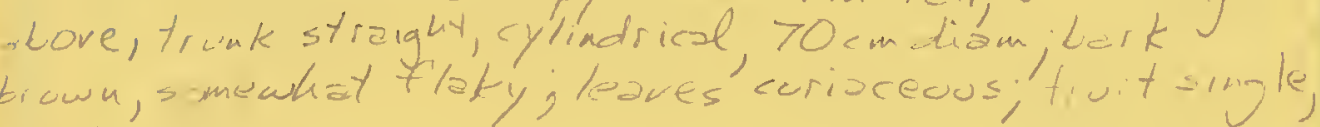
$\therefore=$ ret, green.

Lac: $(3842)$

Lat:: $(=84 z)$ Long.: Alt.:

Habitat: (3842, upper ter ba line)

Lox. names):

Med. uses):

Parts) used:

General notes:

Hazard notes:

Collectors): Mc Donald \& Ismail

No.: $3884 \quad$ Date: 25 f ul 1993

Sample plant parts): $\angle F \quad U 44 Z-7946-\omega$

NCI code numbers): $\begin{array}{ll}\text { To } & -7947-x \\ B A & -7948-y\end{array}$

Collected under the auspices of:

U.S. National Cancer Institute, Bethesda, MD.

University of Illinois at Chicago, Chicago, IL.

Amold Arboretum, Harvard University, Cambridge, MA.

Field Museum of Natural History, Chicago, IL.

Host Institution: 


\section{PLANTS OF}

This is a voucher specimen for anticancer testing: YES NO

Fam.: Lauraceae

$$
\text { Cryptocarya }
$$

Det. by:

Date:

Field characters:

Lat.: $(3842)$ Long.: Alt: :

Habitat: (3812 - uppier timberlais)

Loc. name(s):

Med. use(s):

Part(s) used:

General notes:

Hazard notes:

Collector(s):

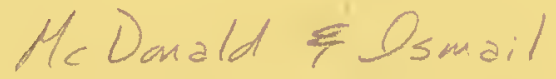

$$
\text { No.: } 3885 \quad \text { Date: } 25 \text { fol } 1993
$$

Sample plant part(s): $\frac{\angle F}{T W} U 44 Z-7949-Z$

NCI code number(s): $-7750-A$

Collected under the auspices of:

$-7951-C$
$-7952-F$

U.S. National Cancer Institute, Bethesda, MD.

University of Illinois at Chicago, Chicago, IL.

- Amold Arboretum, Harvard University, Cambridge, MA.

Field Museum of Natural History, Chicago, IL.

Host Institution: 


\section{PLANTS OF}

This is a voucher specimen for anticancer testing: YES NO

Fam.: The aceae

Ternstroemia

Det. by:

Field characters:

cylindrical,

ight green, kaves coriace

Loc.:

$$
(38 / 2)
$$

Date:

Lat: $(3542)$ Long.: Alt.:

Habitat: ( $35<2$, ypper timberline)

Loc. name(s):

Med. use(s):

Part(s) used:

General notes:

Hazard notes:

Collector(s):

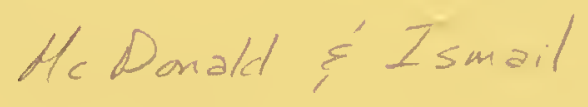

$$
\text { No.: } 3886 \quad \text { Date: } 25 \text { foll } 1993
$$

Sample plant part(s): $\quad \angle F \quad U / 4 / Z-7953-6$

$\mathrm{NCl}$ code number(s):

$$
\text { Tw }
$$

BA

$-7954-H$

$-7955-I$

Collected under the auspices of:

U.S. National Cancer Institute, Bethesda, MD.

University of Illinois at Chicago, Chicago, IL.

Amold Arboretum, Harvard University, Cambridge, MA.

Field Museum of Natural History, Chicago, IL.

Host Institution: 


\section{PLANTS OF}

This is a voucher specimen for anticancer testing: YES $\_$NO

Fam.: Sapotaceae

Planchone//a

Det. by:

Date:

Field characters: Tree $13 \mathrm{~m}$ tall bracking midway up,

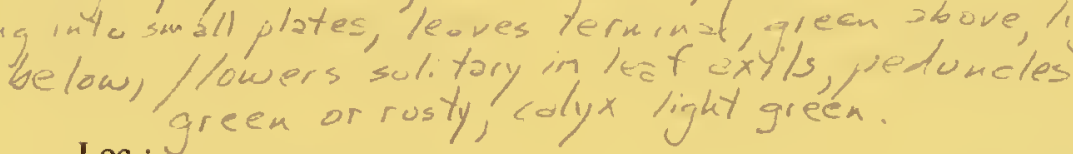

Loc.: $(3542)$

Lat.: $(3 J 4 / 2)$ Long.: Alt.:

Habitat: ( 3542 , upper timberline)

Loc. name(s):

Med. use(s):

Part(s) used:

General notes:

Hazard notes:

Collector(s):

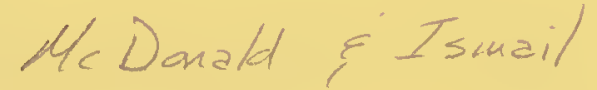

No.: 3887

Date:

Sample plant part(s): $\angle F \quad U \angle 4 Z-7956-J$

$\mathrm{NCl}$ code number(s):

Collected under the auspices of:

U.S. National Cancer Institute, Bethesda, MD.

University of Illinois at Chicago, Chicago, IL.

- Amold Arboretum, Harvard University, Cambridge, MA.

Field Museum of Natural History, Chicago, IL.

Host Institution: $E O \quad \sim / P \mid$ 


\section{PLANTS OF}

This is a voucher specimen for

anticancer testing: YES NO

Det. by:

Date:

Field characters:

Loc.:

Fam.: Arecaceae

Pinanga

Lat: $(3842)$ Long:

Alt.:

Habitat: ( 3842 , upper timberline)

Loc. name(s):

Med. use(s):

Part(s) used:

General notes:

Hazard notes:

Collector(s): He Donald $\xi$ Ismail

$$
\text { No.: } 3888 \quad \text { Date: } 25 \text { fol } 1993
$$

Sample plant part(s): $\angle F \quad U 44 Z-7959-M$

NCI code number(s): $\begin{array}{ll}P E & -7960-N \\ R T & -7961-0\end{array}$

Collected under the auspices of:

U.S. National Cancer Institute, Bethesda, MD.

University of Illinois at Chicago, Chicago, IL.

Arnold Arboretum, Harvard University, Cambridge, MA.

Field Museum of Natural History, Chicago, IL.

Host Institution: $8,5<1,4$ 


\section{PLANTS OF}

This is a voucher specimen for

anticancer testing: YES NO

Fam.: $0 / 4$ aceae

chionanthus

Det. by:

Date:

Field characters: Canopy tree in r-vine, $24 \mathrm{~m}$ tall,
sbove, trunk cylidadrical, $18 \mathrm{~cm}$ di $2 m$, tork bran hew g above, trunk cylindrical, $18 \mathrm{~cm}$ diam, idork
light gray, somewhat roughemed; fruits immature, green,

Loc.: $(3842)$

Lat.: $(3542)$ Long.:

Alt.:

Habitat: ( 3842 , upper tmberline)

Loc. name(s):

Med. use(s):

Part(s) used:

General notes:

Hazard notes:

Collector(s):

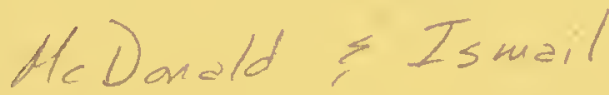

No.: $3889 \quad$ Date: 25 fol 1993
Sample plant part(s): $\angle F \quad U 44 Z-7962-P$

NCI code number(s):

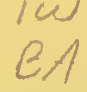$$
-7963-Q
$$$$
-7964-R
$$

Collected under the auspices of:

U.S. National Cancer Institute, Bethesda, MD.

University of Illinois at Chicago, Chicago, IL.

- Amold Arboretum, Harvard University, Cambridge, MA.

Field Museum of Natural History, Chicago, IL.

Host Institution: 


\section{PLANTS OF}

This is a voucher specimen for

anticancer testing: YES NO

Fam.: Clusiaceae

$$
\text { Calophyllum } \quad \text { esterile }=
$$

Det. by:

Date:

Field characters: $M$ instery tree 12 m tall, Lumcheng

Loc.: $(38 \% 2)$

Lat.: $(3842)$ Long.:

Alt.:

Habitat: $(3842)$

Loc. name(s):

Med. use(s):

Part(s) used:

General notes:

Hazard notes:

Collector(s): $/<D$ anald $E I \sin 21 /$

$$
\text { No: } 3890 \text { Date: } 25 \mathrm{fol} / 993
$$

Sample plant part(s): LF U44Z-7965-

$\mathrm{NCI}$ code number(s):

$$
\text { Tw }
$$

BA

$-7966-T$

Collected under the auspices of:

U.S. National Cancer Institute, Bethesda, MD.

University of Illinois at Chicago, Chicago, IL.

- Amold Arboretum, Harvard University, Cambridge, MA.

Field Museum of Natural History, Chicago, IL.

Host Institution: 


\section{PLANTS OF}

This is a voucher specimen for

anticancer testing: YES

$\leftarrow$ NO

Fam.: U/maceae

Trema

Det. by:

Date:

Field characters:

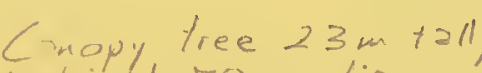

and below; truale cylientrical

hark light glay, smoothish

green, stigromahite.

Loc.: $(33 / 2)$

Lat.: $(38 \% / 2)$ Long.:

Alt:

Habitat: $(3842)$

Loc. name(s):

Med. use(s):

Part(s) used:

General notes:

Hazard notes:

Collector(s):

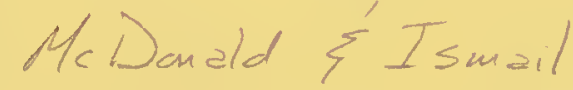

$$
\text { No.: } 3892 \text { Date: } 26 \text { ful } 1993
$$

Sample plant part(s): $\quad \angle F \quad \cup 44 Z-7965-V$

NCI code number(s): $\begin{array}{ll}\text { Tw } & -7969-\omega \\ B A & -7970-x\end{array}$

Collected under the auspices of:

U.S. National Cancer Institute, Bethesda, MD.

University of Illinois at Chicago, Chicago, IL.

- Amold Arboretum, Harvard University, Cambridge, MA.

Field Museum of Natural History, Chicago, IL.

Host Institution: 


\section{PLANTS OF}

This is a voucher specimen for anticancer testing: YES NO

Fam.: Laurace
Dehasia

Det. by:

Date:

Field characters: joy, weakly wertical-furrowed; foliage coria
green; thowers lighy green, stemens yellow.

Loc: $(384 / 2)$

Lat.: $(3542)$ Long.:

Alt:

Habitat: $(3842)$

Loc. name(s):

Med. use(s):

Part(s) used:

General notes:

Hazard notes:

Collector(s):

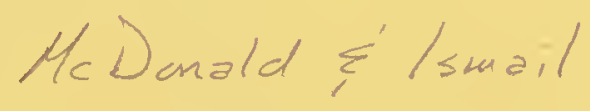

$$
\text { No.: } 3893 \text { Date: } 26 \text { /ull } 1993
$$

Sample plant part(s): $\quad \angle F \quad \cup 44 z-797 /-Y$

NCI code number(s): $\begin{array}{ll}T \omega & -7972-Z \\ B A & -7973-A\end{array}$

Collected under the auspices of:

U.S. National Cancer Institute, Bethesda, MD.

University of Illinois at Chicago, Chicago, IL.

- Arnold Arboretum, Harvard University, Cambridge, MA. Field Museum of Natural History, Chicago, IL.

Host Institution: 


\section{PLANTS OF}

This is a voucher specimen for

anticancer testing: YES NO

Fam.: Lauraceae

De hasia

Det. by:

Date:

Field characters:

Lat:: $(382 / 2)$ Long.:

Alt: :

Habitat: $(3842)$

Loc. name(s):

Med. use(s):

Part(s) used:

General notes:

Hazard notes:

Collector(s):

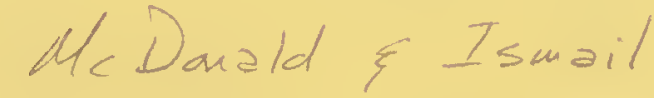

No.: 3894 Date: 26 foel 1943

Sample plant part(s): LF U/4Z-7974-C

NCI code number(s):
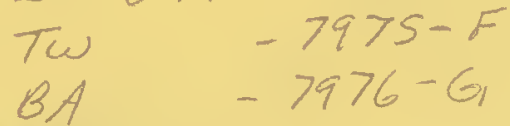

Collected under the auspices of:

U.S. National Cancer Institute, Bethesda, MD.

University of Illinois at Chicago, Chicago, IL.

Amold Arboretum, Harvard University, Cambridge, MA.

Field Museum of Natural History, Chicago, IL.

Host Institution: 50 


\section{PLANTS OF}

This is a voucher specimen for anticancer testing: YES NO

Fam.: Hyristicaceae Kn $\ll$ ma

Det. by:

Tree II mate:

Field characters:

and belew

itruak

green abore, silverish below,

Loc: $(384 / 2)$

Lat:: $(3842)$ Long.:

Alt.:

Habitat: $(38</ 2)$

Loc. name(s):

Med. use(s):

Part(s) used:

General notes:

Hazard notes:

Collector(s):

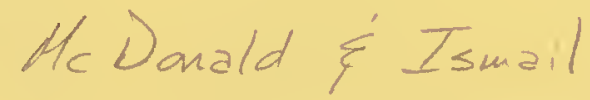

$$
\text { No.: } 3895 \quad \text { Date: } 26 \text { ful } 1993
$$

Sample plant part(s): $\quad \angle F \quad U 4 / 4 Z-7977-H$

$\mathrm{NCl}$ code number(s):

$$
\text { Tw }
$$

$B A$

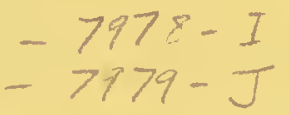

Collected under the auspices of:

U.S. National Cancer Institute, Bethesda, MD.

University of Illinois at Chicago, Chicago, IL.

- Amold Arboretum, Harvard University, Cambridge, MA.

Field Museum of Natural History, Chicago, IL.

Host Institution: 
This is a voucher specimen for anticancer testing: YES _ NO

Fam.:

Det. by:

Date:

Field characters:

Loc.:

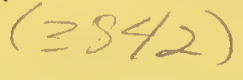

Lat.: $(38 / 2)$ Long.:

Alt:

Habitat:

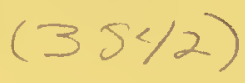

Loc. name(s):

Med. use(s):

Part(s) used:

General notes:

Hazard notes:

Collector(s):

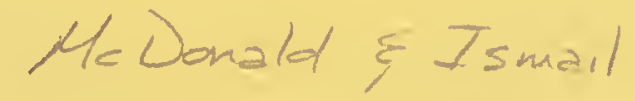

No.: 3896 Date:

Sample plant part(s): $\angle F \quad U / 4 / Z-7930-K$

$\mathrm{NCI}$ code number(s):

$T_{B A}$

$-7981-\angle$

Collected under the auspices of:

U.S. National Cancer Institute, Bethesda, MD.

University of Illinois at Chicago, Chicago, IL.

Amold Arboretum, Harvard University, Cambridge, MA.

Field Museum of Natural History, Chicago, IL.

Host Institution: $B=, \angle i r$ 


\section{PLANTS OF}

This is a voucher specimen for anticancer testing: YES $\measuredangle$ NO

Fam.: Ebenaceae

$$
\text { Diospyros }
$$

Det. by:
Field characters: Short canopy tree $13 \mathrm{~m}$ tall, branchm
unk scmewh at crooked, $15 \mathrm{~cm}$ liam; bark gray, shy

Det. by:
Field characters: Short camopy tree $13 \mathrm{~m}$ tall, branchm
unk scmewh at crooked, $15 \mathrm{~cm}$ liam; bark gray, shy atsore, trunk scmewhat in er bark yellow, Gaves tar'k green above, stmoothish, below; flowers caulf flosous anlateral branches maturing fruits silvery green - velutinous

Loc: $(3842)$

Lat: $(3342)$ Long.:

Alt : :

Habitat: $384 / 2)$

Loc. name(s):

Med. use(s):

Part(s) used:

General notes:

Hazard notes:

Collector(s):

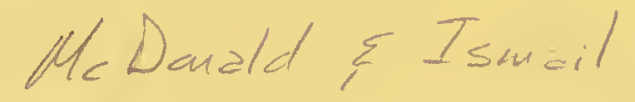

$$
\text { No.: } 5897 \text { Date: } 26 \text { Pal /993 }
$$

Sample plant part(s): LF U4HZ $-7983-N$

NCI code number(s): Tw $-7984-0$

$$
B A-7985-p
$$

Collected under the auspices of:

U.S. National Cancer Institute, Bethesda, MD.

University of Illinois at Chicago, Chicago, IL.

- Arnold Arboretum, Harvard University, Cambridge, MA.

Field Museum of Natural History, Chicago, IL. 


\section{PLANTS OF}

This is a voucher specimen for anticancer testing: YES NO

Fam.: U/maceae Girroniera

Det. by:

Date:

Field characters:

Lat: $(3842)$ Long.:

Alt.:

Habitat:

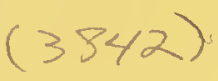

Loc. name(s):

Med. use(s):

Part(s) used:

General notes:

Hazard notes:

Collector(s):

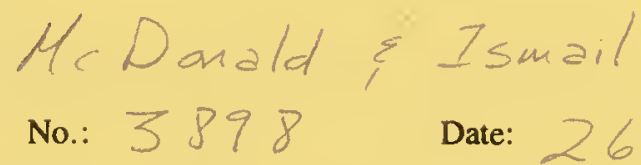

Sample plant part(s): $\angle F \quad \cup 44 Z-7986-Q$

$\mathrm{NCI}$ code number(s):
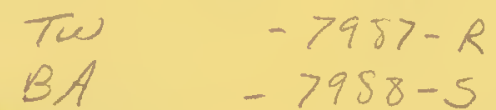

$-7958-5$

Collected under the auspices of:

U.S. National Cancer Institute, Bethesda, MD.

University of Illinois at Chicago, Chicago, IL.

- Arnold Arboretum, Harvard University, Cambridge, MA.

Field Museum of Natural History, Chicago, IL.

Host Institution: 


\section{PLANTS OF}

This is a voucher specimen for anticancer testing: YES NO

Fam.: Burseracede

Det. by:

Date:

Field characters:

Lat: $(38 / 2)$ Long.:

Alt.:

Habitat: $(3842)$

Loc. name(s):

Med. use(s):

Part(s) used:

General notes:

Hazard notes:

Collector(s):

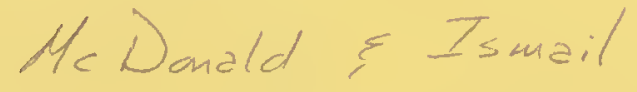

$$
\text { No.: } 3899 \text { Date: } 26 \text { ful } / 993
$$

Sample plant part(s): $\angle F \quad U S / 4 Z-7789-T$

$\mathrm{NCl}$ code number(s):

Collected under the auspices of:

Fpices of

U.S. National Cancer Institute, Bethesda, MD.

University of Illinois at Chicago, Chicago, IL.

Amold Arboretum, Harvard University, Cambridge, MA.

Field Museum of Natural History, Chicago, IL.

Host Institution: 


\section{PLANTS OF}

This is a voucher specimen for

anticancer testing: YES NO

Fam.:

Det. by:

Date:

Field characters:

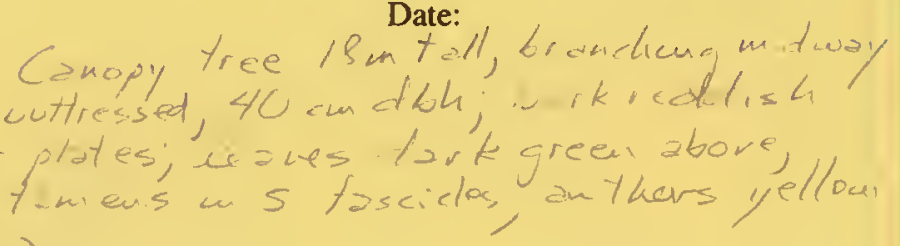

Loc: $(334 / 2)$

Lat.: $(35 \%$ Long.:

Alt.:

Habitat:

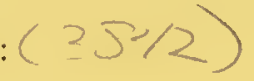

Loc. name(s):

Med. use(s):

Part(s) used:

General notes:

Hazard notes:

Collector(s):

$$
\text { No.: }
$$

Date:

Sample plant part(s): $\quad U 44 Z-7995-x$

$\mathrm{NCl}$ code number(s):

Collected under the auspices of:

U.S. National Cancer Institute, Bethesda, MD.

University of Illinois at Chicago, Chicago, IL.

Arnold Arboretum, Harvard University, Cambridge, MA.

Field Museum of Natural History, Chicago, IL.

Host Institution: 


\section{PLANTS OF}

This is a voucher specimen for anticancer testing: YES $\checkmark \mathrm{NO}$

Fam.: Sterculiaceae

Det. by:

Date:

beluw frunk cylinatrical, $20 \mathrm{~cm}$

zantal, black lenticels;
olla throat orange,
Loc.: $(33</ 2)$

Lat.: $(\geq: 1 / 2)$ Long.:

Alt.:

Habitat: $(38,8 / 2)$

Loc. name(s):

Med. use(s):

Part(s) used:

General notes:

Hazard notes:

Collector(s):

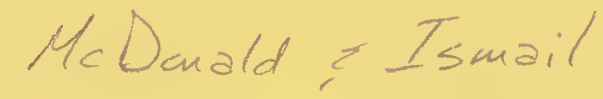

No.:

Date:

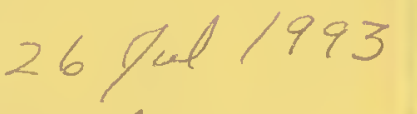

Sample plant part(s): $\angle F \quad \cup 44 Z-7996-1$

$\mathrm{NCl}$ code number(s):

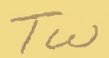

$B A$

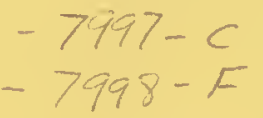

Collected under the auspices of:

U.S. National Cancer Institute, Bethesda, MD.

University of Illinois at Chicago, Chicago, IL.

Amold Arboretum, Harvard University, Cambridge, MA.

Field Museum of Natural History, Chicago, IL.

Host Institution: 


\section{PLANTS OF}

This is a voucher specimen for anticancer testing: YES NO

Fam.: O/acaceae

Strombosia

Det. by:

Date:

Field characters:

Field characers:

,

runk straight?

Loc: $(3842)$

Lat.: $(2842)$ Long.:

Alt.:

Habitat:

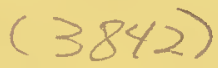

Loc. name(s):

Med. use(s):

Part(s) used:

General notes:

Hazard notes:

Collector(s): He Danald Ismail

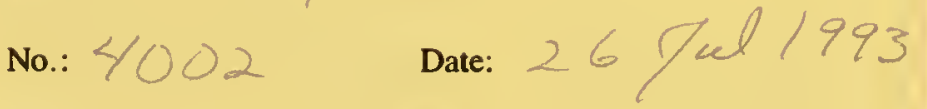

Sample plant part(s): $\angle F \quad U 44 Z-7999-G$

NCI code number(s): Tw $B A$ $-8000-H$ - $8001-I$

Collected under the auspices of:

U.S. National Cancer Institute, Bethesda, MD.

University of Illinois at Chicago, Chicago, IL.

Amold Arboretum, Harvard University, Cambridge, MA.

Field Museum of Natural History, Chicago, IL.

Host Institution: 


\section{PLANTS OF}

This is a voucher specimen for anticancer testing: YES

Fam.: Jterculiaceae

$$
\text { Pterospermum }
$$

Det. by:

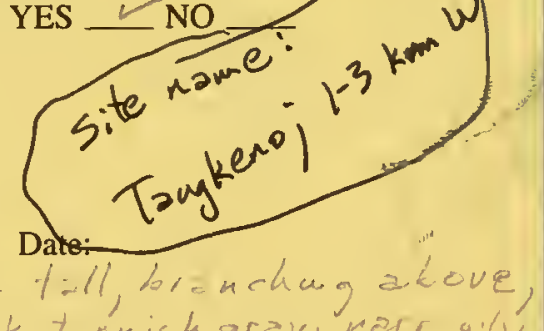

Field characters:

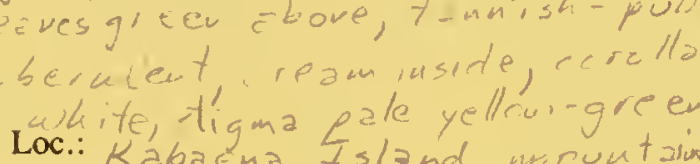

ght, eylibedoual

oc.: Kabaema Island, mountaim ri lge between

$$
\text { Lat.: } 5^{\circ} 16^{\prime} S \text { Long.: } 121^{\circ} 56^{\prime} E \text { Alt.: } 700-800 \mathrm{~m}
$$

Habitat:

$$
\text { Secon tary forest around ladangs. }
$$

tes include Arenga, bamboos, Ficus,

Trickospermum, Albizia, ete.

Loc. name(s):

Med. use(s):

Part(s) used:

General notes:

Hazard notes:

Collector(s):

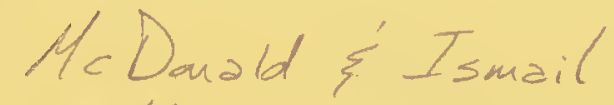

$$
\text { No.: } 4004 \text { Date: } 27 \text { Pal } 1993
$$

Sample plant part(s): $\angle F \quad U 4 / Z Z-8002-J$

NCI code number(s):

$$
\text { Tw }
$$

$B A$

$-8003-K$

$-8004-L$

Collected under the auspices of:

U.S. National Cancer Institute, Bethesda, MD.

University of Illinois at Chicago, Chicago, IL.

Amold Arboretum, Harvard University, Cambridge, MA.

Field Museum of Natural History, Chicago, IL.

Host Institution: 


\section{PLANTS OF}

This is a voucher specimen for anticancer testing: YES NO

Fam.: M.mo
$?$ Albizia

Det. by:

Date:

Field characters: Shor tree $7 \mathrm{~m}$ tall, bianchuig above bekw; trurt cylim-rical, Bcw tiam; tark garayish smooth; truits green, mottledred

Loc: $(4004)$

Lat.: $(400 \%)$ Long.:

Alt: :

Habitat:

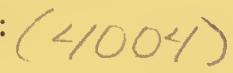

Loc. name(s):

Med. use(s):

Part(s) used:

General notes:

Hazard notes:

Collector(s):

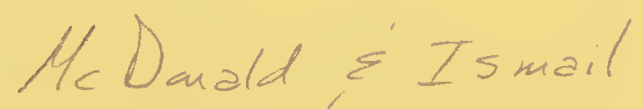

No.: $4005 \quad$ Date: 27

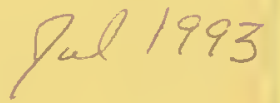

Sample plant part(s): LF U44Z-8005-M

NCI code number(s):

Collected under the auspices of:

U.S. National Cancer Institute, Bethesda, MD.

University of Illinois at Chicago, Chicago, IL.

- Amold Arboretum, Harvard University, Cambridge, MA. Field Museum of Natural History, Chicago, IL.

Host Institution: 


\section{PLANTS OF}

This is a voucher specimen for anticancer testing: YES NO

Fam.: Tilia ceae

Trichospermum

Det. by:

Date:

Field characters: Short tiee
telcas; trunt 30 mol tiam,

le gray, =mooth

Loc.: $(4004)$

Lat.: $(4004)$ Long.:

Alt. :

Habitat: $(4004)$

Loc. name(s):

Med. use(s):

Part(s) used:

General notes:

Hazard notes:

Collector(s):

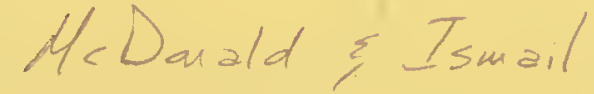

No.: $4006 \quad$ Date: 27

Sample plant part(s):

$U 44 Z-8008-P$

$\mathrm{NCI}$ code number(s):

Collected under the auspices of:

U.S. National Cancer Institute, Bethesda, MD.

University of Illinois at Chicago, Chicago, IL.

Amold Arboretum, Harvard University, Cambridge, MA.

Field Museum of Natural History, Chicago, IL.

Host Institution: 


\section{PLANTS OF}

This is a voucher specimen for anticancer testing: YES _ NO

Fam.: Hor aceae

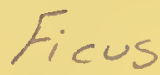

Det. by:

Date:

Field characters: "anopy toee $20 \mathrm{~m}$ tall, brameluarg

up trunk cylinitrical, crooked, 20 cm bam

cinnsh gray, smoothish; lecups scabrous

Loc:

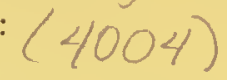

Lat: $(4004)$ Long.:

Alt.:

Habitat: $(4004)$

Loc. name(s):

Med. use(s):

Part(s) used:

General notes:

Hazard notes:

Collector(s):
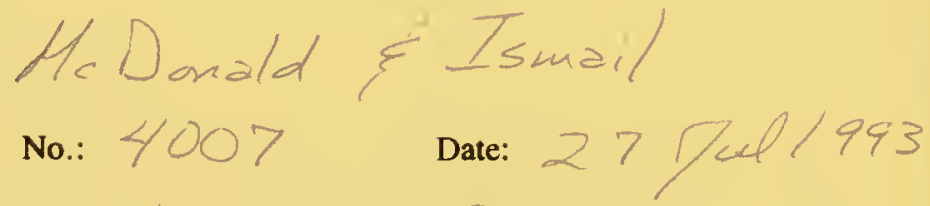

Sample plant part(s): $\angle F \cup 44 z-8011-5$

NCI code number(s):

$$
\text { Tw }
$$

Collected under the auspices of:

U.S. National Cancer Institute, Bethesda, MD.

University of Illinois at Chicago, Chicago, IL.

- Amold Arboretum, Harvard University, Cambridge, MA.

Field Museum of Natural History, Chicago, IL.

Host Institution: 


\section{PLANTS OF}

This is a voucher specimen for anticancer testing: YES NO

Fam.: Acanthaceae

Det. by:

Date:

Field characters: bace, Zn yall,

lavender usprtect

Loc: $(4004)$

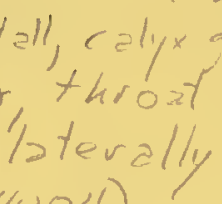

trom

$$
\text { Understory shrub }
$$

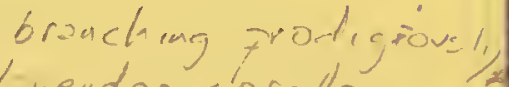

Lat.: (4/004) Long.:

Alt:

Habitat:

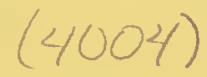

Loc. name(s):

Med. use(s):

Part(s) used:

General notes:

Hazard notes:

Collector(s):
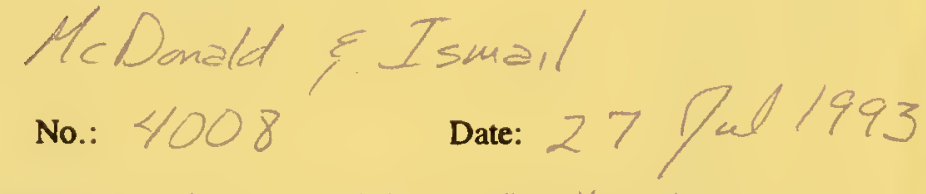

Sample plant part(s): LF UH/4Z-80/4-V

NCI code number(s): Tw $\quad-8015-W$

Collected under the auspices of:

U.S. National Cancer Institute, Bethesda, MD.

University of Illinois at Chicago, Chicago, IL.

Amold Arboretum, Harvard University, Cambridge, MA.

Field Museum of Natural History, Chicago, IL.

Host Institution: 


\section{PLANTS OF}

This is a voucher specimen for anticancer testing: YES $\sim$ NO

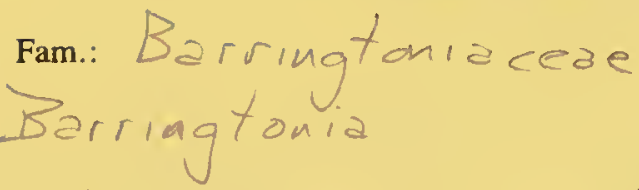

Det. by:

Date:

Field characters:

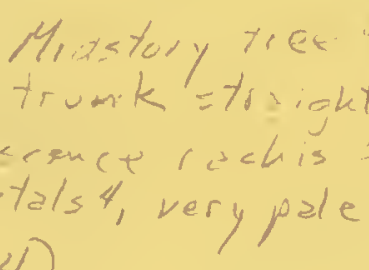

Loc.: $(4004)$

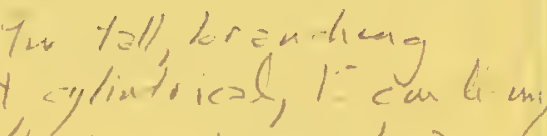

Lat: $(400 \%)$ Long.:

Alt.:

Habitat:

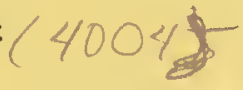

Loc. name(s):

Med. use(s):

Part(s) used:

General notes:

Hazard notes:

Collector(s):

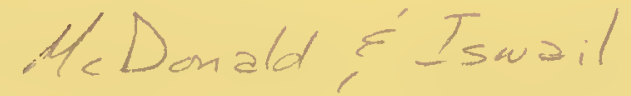

No.: 4009

Date:

Sample plant part(s): $\angle F \quad U 44 Z-8016-X$

NCI code number(s):

Collected under the auspices of:

U.S. National Cancer Institute, Bethesda, MD.

University of Illinois at Chicago, Chicago, IL.

Amold Arboretum, Harvard University, Cambridge, MA.

Field Museum of Natural History, Chicago, IL.

Host Institution: 


\section{PLANTS OF}

This is a voucher specimen for anticancer testing: YES $\simeq$ NO

Fam.: Caesalpin.aceae

Det. by:

Date:

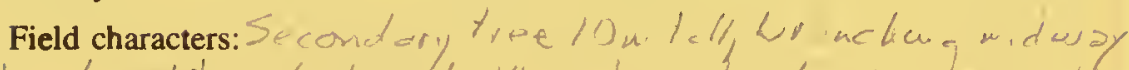

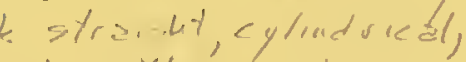
ni nior bark yellow, calyx

Loc.: $(4004)$

Lat.: $(4004)$ Long.:

Alt.:

Habitat: $(204)$

Loc. name(s):

Med. use(s):

Part(s) used:

General notes:

Hazard notes:

Collector(s):

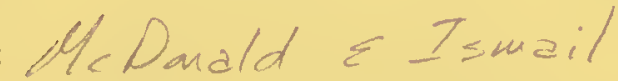

No.: $4 / 010$

Date: 27 Dal $/ 993$

Sample plant part(s): LF U4/ $/ Z-8019-A$

$\mathrm{NCI}$ code number(s):

Collected under the auspices of:

U.S. National Cancer Institute, Bethesda, MD.

University of Illinois at Chicago, Chicago, IL.

Amold Arboretum, Harvard University, Cambridge, MA.

Field Museum of Natural History, Chicago, IL.

Host Institution: 


\section{PLANTS OF}

This is a voucher specimen for anticancer testing: YES

Fam.: Rubi aceae

Det. by:

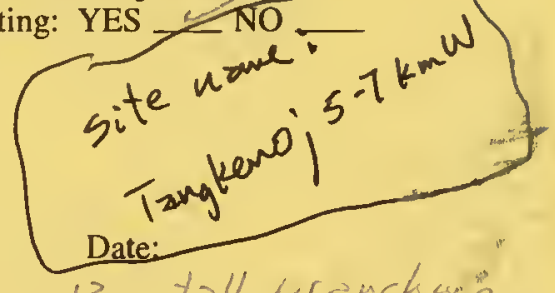

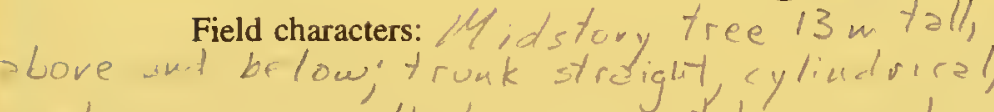
tok cream ciolth borewn fivits imarature, green.

Loc: Kabaena Islant, Botu Sangia peak, $5-7 \mathrm{~km}$ Wof Tangkeno.

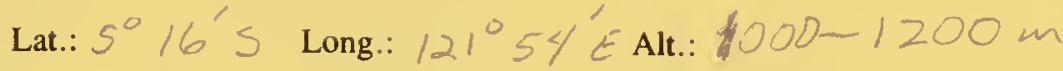
Habitat: Forest below clifts, Eslopes, Mesophytic, Hssociates cri clude hithocarpos, Eugenia, Voacangd, Lepisanthes, Celastraceae.

Loc. name(s):

Med. use(s):

Part(s) used:

General notes:

Hazard notes:

Collector(s):

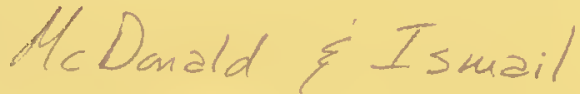

No.: $4011 \quad$ Date: $27 \mathrm{Pul} / 993$

Sample plant part(s): $\angle F \quad$ U44Z - 8022- G

NCI code number(s): Tw $\quad-8023-\mathrm{H}$

Collected under the auspices of:

U.S. National Cancer Institute, Bethesda, MD.

University of Illinois at Chicago, Chicago, IL.

- Amold Arboretum, Harvard University, Cambridge, MA. Field Museum of Natural History, Chicago, IL.

Host Institution: 


\section{PLANTS OF}

This is a voucher specimen for anticancer testing: YES NO

Fam.: Sapindaceae ? Nephelium

Det. by:

Date:

Field characters:

Loc.: $(4011)$ straight

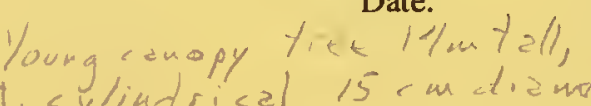

uove,

rimk

mewh?

vipery-pulescert,

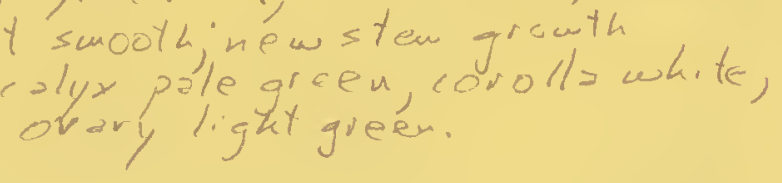

Lat.: $(4011)$ Long.:

Alt :

Habitat:

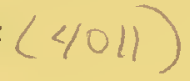

Loc. name(s):

Med. use(s):

Part(s) used:

General notes:

Hazard notes:

Collector(s): McDonald $\sum$ Ismail

$$
\text { No.: } 4012 \quad \text { Date: } 27 \text { Jul } / 993
$$

Sample plant part(s): $\angle F \quad U 44 Z-8025-J$

$\mathrm{NCl}$ code number(s):

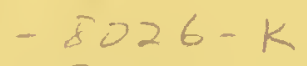

$B A$

$-8027-4$

Collected under the auspices of:

U.S. National Cancer Institute, Bethesda, MD.

University of Illinois at Chicago, Chicago, IL.

Amold Arboretum, Harvard University. Cambridge, MA.

Field Museum of Natural History, Chicago, IL.

Host Institution: 


\section{PLANTS OF}

This is a voucher specimen for anticancer testing: YES NO

Fam.: Fabaceae

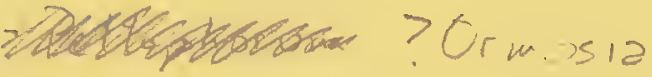

Det. by:

Date:

Field characters:

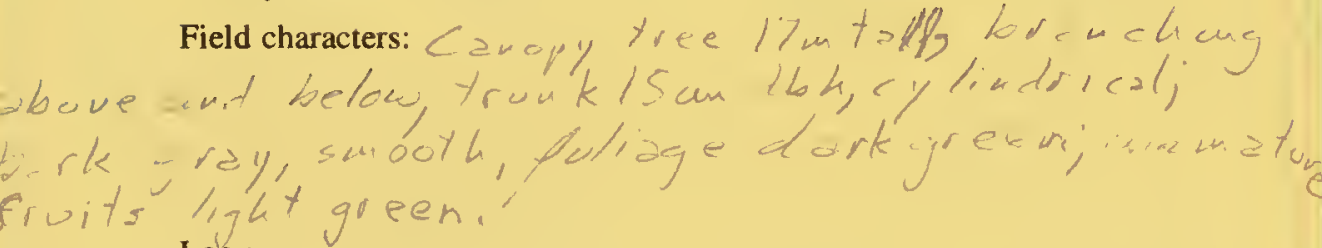

Loc.:

$(40 \|)$

Lat.: $(1011)$ Long.:

Alt.:

Habitat:

\section{$(4011)$}

Loc. name(s):

Med. use(s):

Part(s) used:

General notes:

Hazard notes:

Collector(s): $4<$ Donald $\xi$ Ismail

$$
\text { No.: } 2 / 013 \quad \text { Date: } 27
$$

Sample plant part(s): $\angle F \quad V 44 Z-8028-M$

NCI code number(s): $B A$ _ $8030-$

Collected under the auspices of:

U.S. National Cancer Institute, Bethesda, MD.

University of Illinois at Chicago, Chicago, IL.

- Amold Arboretum, Harvard University, Cambridge, MA.

Field Museum of Natural History, Chicago, IL.

Host Institution: 


\section{PLANTS OF}

This is a voucher specimen for anticancer testing: YES $\longleftarrow$ NO

Fam.: Myrtaceae Eugenia

Det. by:

Date:

Field characters:

akove ant belcu, truak straight,

Loc.: (4011)

2 m $y=11$, wis anclice. ming sm

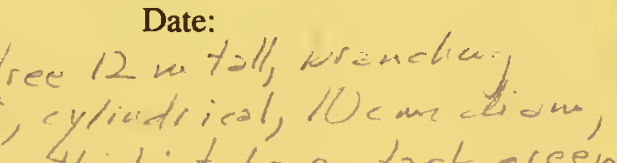

Lat.: $(40 / 1) \quad$ L
Habitat: $(4011)$

Loc. name(s):

Med. use(s):

Long.:

Alt:

Part(s) used:

General notes:

Hazard notes:

Collector(s):

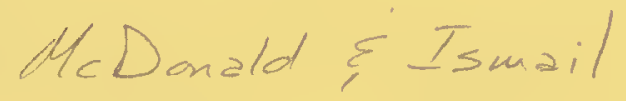

No.: 4014 Date: $27 \mathrm{fal} / 993$

Sample plant part(s): $\angle F \quad U 44 Z-8032-Q$

NCI code number(s):

$$
\begin{array}{ll}
T \omega & -8033-R \\
B A & -8034-S
\end{array}
$$

Collected under the auspices of:

U.S. National Cancer Institute, Bethesda, MD.

University of Illinois at Chicago, Chicago, IL.

Amold Arboretum, Harvard University, Cambridge, MA.

Field Museum of Natural History, Chicago, IL.

Host Institution: 


\section{PLANTS OF}

This is a voucher specimen for anticancer testing: YES NO

Fam.: Moraceae<smiles>[CH]1C[CH-][AsH]1</smiles>

Det. by:

Date:

Field characters:

midway up, trunk cyliadrical,

Loc.:

Lat: $(4011)$ Long.:

Alt.:

Habitat: 4011$)$

Loc. name(s):

Med. use(s):

Part(s) used:

General notes:

Hazard notes:

Collector(s):

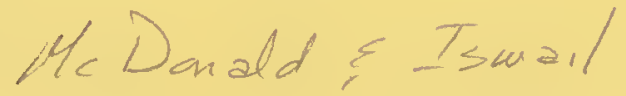

No.: $4015 \quad$ Date: 28 Pal 1993

Sample plant part(s): $\angle F U 4 / Z-8035-T$

$\mathrm{NCl}$ code number(s):

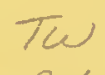

$B A$

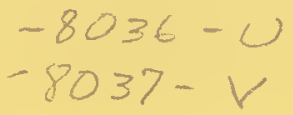

Collected under the auspices of:

U.S. National Cancer Institute, Bethesda, MD.

University of Illinois at Chicago, Chicago, IL.

- Arnold Arboretum, Harvard University, Cambridge, MA.

Field Museum of Natural History, Chicago, IL.

Host Institution: 


\section{PLANTS OF}

This is a voucher specimen for anticancer testing: YES $\measuredangle$ NO

Fam.: Sapinctaceae

?) Elatostachys

Det. by:

Date:

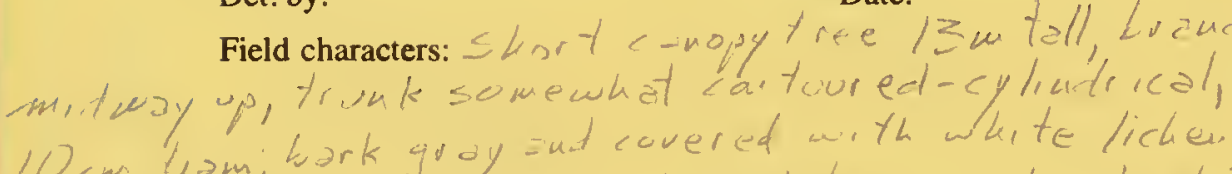

Loc.:

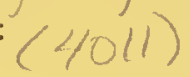

Lat.: $(1 / 011)$ Long.:

Alt.:

Habitat: $4 / 011)$

Loc. name(s):

Med. use(s):

Part(s) used:

General notes:

Hazard notes:

Collector(s):

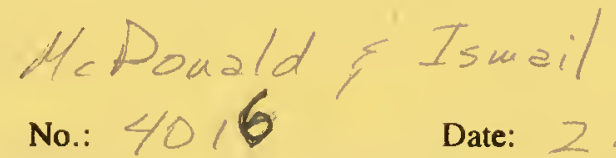

Sample plant part(s): $\angle F$

$U 44 z-8038-\omega$

$\mathrm{NCI}$ code number(s):

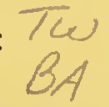

Collected under the auspices of:

U.S. National Cancer Institute, Bethesda, MD.

University of Illinois at Chicago, Chicago, IL.

Amold Arboretum, Harvard University, Cambridge, MA.

Field Museum of Natural History, Chicago, IL.

Host Institution: 


\section{PLANTS OF}

This is a voucher specimen for anticancer testing: YES NO

Fam.: Lauraceae

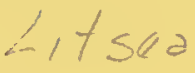

Det. by:

Date:

Field characters: Mitstory to ee Ilu toll, bercuckurg abovej trink rreqular, SOcandiam; hark brown wilh

Loc.:<smiles>[GeH3]</smiles>

Lat.: $(4 / 1 / 1) \quad$ Long.:

Alt.:

Habitat: $(40 / 1)$

Loc. name(s):

Med. use(s):

Part(s) used:

General notes:

Hazard notes:

Collector(s):
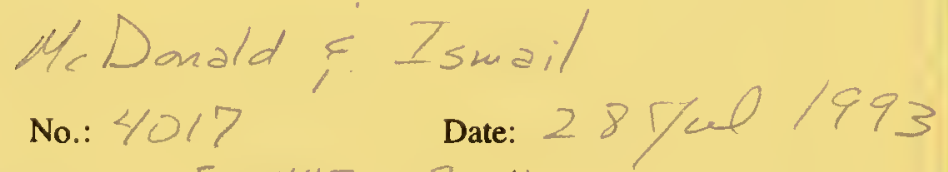

Sample plant part(s): $\begin{array}{ll}\angle F U 44 Z & -8041-Z \\ T \omega & -8042-A\end{array}$

NCI code number(s): $B A \quad-8043-C$

Collected under the auspices of:

$-8044-F$

U.S. National Cancer Institute, Bethesda, MD.

University of Illinois at Chicago, Chicago, IL.

Amold Arboretum, Harvard University, Cambridge, MA.

Field Museum of Natural History, Chicago, IL.

Host Institution: 


\section{PLANTS OF}

This is a voucher specimen for anticancer testing: YES $\_$NO

Fam.: Moraceae

Ficus

Det. by:

Date:

Field characters: $\angle a r g e$

toum base and above, $10 \mathrm{~m}$ tall

cylicitical, Zucwatiam,

succulent-coriaceous, tigs cream.

Loc.: $(4011)$

Lat.: $(4011) \quad$ Long.:

Alt.:

Habitat: $(4011)$

Loc. name(s):

Med. use(s):

Part(s) used:

General notes:

Hazard notes:

Collector(s):

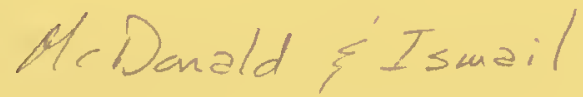

$$
\text { No.: } 4 / 018 \text { Date: } 28 \mathrm{Tul} / 993
$$

Sample plant part(s): $\angle F \quad U 4 / Z-8045-G$

$\mathrm{NCI}$ code number(s):

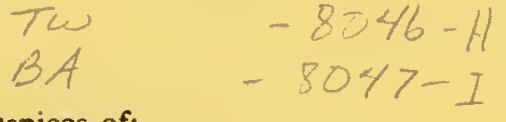

Collected under the auspices of:

U.S. National Cancer Institute, Bethesda, MD.

University of Illinois at Chicago, Chicago, IL.

- Amold Arboretum, Harvard University, Cambridge, MA.

Field Museum of Natural History, Chicago, IL.

Host Institution: 


\section{PLANTS OF}

This is a voucher specimen for anticancer testing: YES NO

Fam.: Rubiaceae

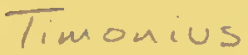

Det. by:

Field characters:

fruits
Loc.: $(4 / \mathrm{U} / 1)$

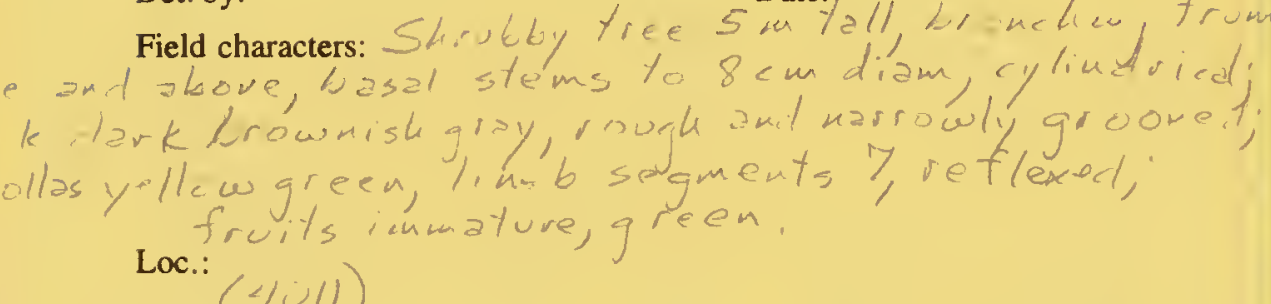

Lat.: $(40 / 1)$ Long.:

Alt :

Habitat: $(1 / 2 / 1)$

Loc. name(s):

Med. use(s):

Part(s) used:

General notes:

Hazard notes:

Collector(s):

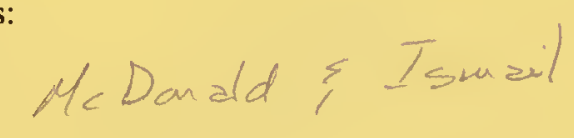

No.: $4019 \quad$ Date: 28 f fwl 1993

Sample plant part(s): $\angle F \quad U 44 Z-8048-J$

$\mathrm{NCl}$ code number(s):

$$
\text { Tie }
$$$$
-8049-K
$$$$
-8050-4
$$

Collected under the auspices of:

U.S. National Cancer Institute, Bethesda, MD.

University of Illinois at Chicago, Chicago, IL.

Amold Arboretum, Harvard University, Cambridge, MA.

Field Museum of Natural History, Chicago, IL.

Host Institution: 


\section{PLANTS OF}

This is a voucher specimen for anticancer testing: YES NO

Fam.: Celastracea e

Det. by:

Date:

Field characters:

Lat.: $(4 / 1)$ Long.:

Alt:

Habitat: $(/ 0 / 1)$

Loc. name(s):

Med. use(s):

Part(s) used:

General notes:

Hazard notes:

Collector(s):
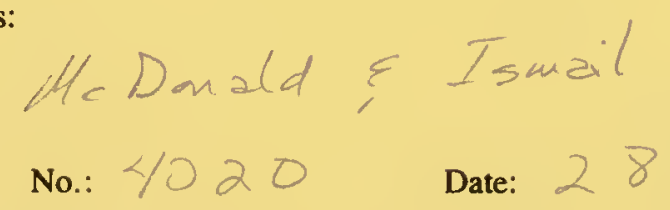

Sample plant part(s): LF U4/ZZ- $2051-M$

NCI code number(s):

Collected under the auspices of:

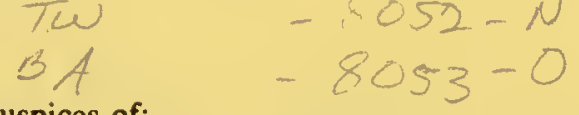

U.S. National Cancer Institute, Bethesda, MD.

University of Illinois at Chicago, Chicago, IL.

Amold Arboretum, Harvard University, Cambridge, MA

Field Museum of Natural History, Chicago, IL.

Host Institution: 


\section{PLANTS OF}

This is a voucher specimen for

Fam.: ? anticancer testing: YES

Det. by:

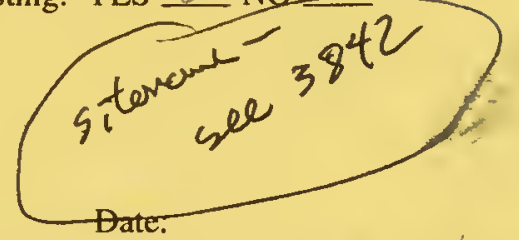

Field characters:

$3 \mathrm{~cm}$ tiam, bark pale tan-gray,

green; fru,ts immature, green, spongy.

Loc.: $(3542)$

Lat.: $(3842)$ Long.:

Alt: :

Habitat: $<342$

Loc. name(s):

Med. use(s):

Part(s) used:

General notes:

Hazard notes:

Collector(s):

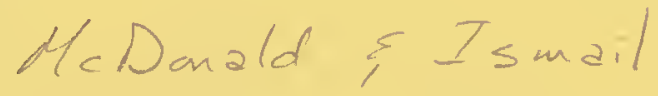

No.: 4021 Date: $29 \mathrm{Tul} / 993$

Sample plant part(s): $\quad \angle F \quad \cup 44 Z-2054-P$

$\mathrm{NCI}$ code number(s):

$$
\text { Tw } \quad-8055-Q
$$

Collected under the auspices of:

U.S. National Cancer Institute, Bethesda, MD.

University of Illinois at Chicago, Chicago, IL.

Amold Arboretum, Harvard University, Cambridge, MA.

Field Museum of Natural History, Chicago, IL.

Host Institution: 


\section{PLANTS OF}

This is a voucher specimen for anticancer testing: YES NO

Fam.: ffyristicaceae

Myristica

Det. by:

Date:

Field characters:

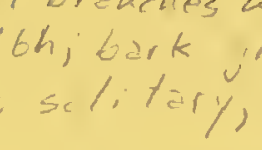

Loc.

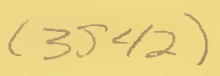

Lat.: $(354 / 2)$ Long.:

Alt.:

Habitat:

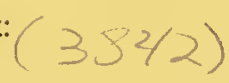

Loc. name(s):

Med. use(s):

Part(s) used:

General notes:

Hazard notes:

Collector(s):

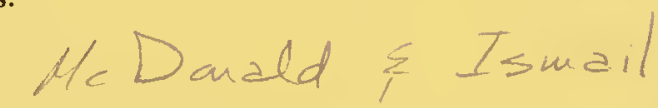

$$
\text { No.: } 4022 \text { Date: } 29
$$

Sample plant part(s): $\angle F \cup 44 Z-805 L-R$

$\mathrm{NCI}$ code number(s):

$$
\text { Tw }
$$

$B A$

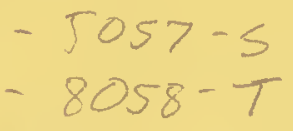

Collected under the auspices of:

U.S. National Cancer Institute, Bethesda, MD.

University of Illinois at Chicago, Chicago, IL.

- Amold Arboretum, Harvard University, Cambridge, MA.

Field Museum of Natural History, Chicago, IL.

Host Institution: 


\section{PLANTS OF}

This is a voucher specimen for

anticancer testing: YES NO

Fam.: Kubiaceae

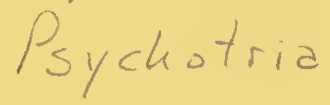

Det. by:

Date:

Field characters: Small, margual-for est toee, 5 m tel/,

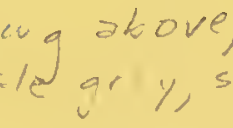

Loc.: $(3542)$

Lat: $(3842)$ Long.:

Alt :

Habitat: $(35</ 2)$

Loc. name(s):

Med. use(s):

Part(s) used:

General notes:

Hazard notes:

Collector(s):

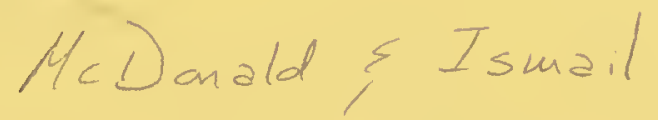

No.: $4023 \quad$ Date: $291 / 001993$

Sample plant part(s): $\angle F U 44 Z-8.359-U$

$\mathrm{NCl}$ code number(s):

$$
\text { Tw }
$$

$B A$

Collected under the auspices of:

U.S. National Cancer Institute, Bethesda, MD.

University of Illinois at Chicago, Chicago, IL.

Arnold Arboretum, Harvard University, Cambridge, MA.

Field Museum of Natural History, Chicago, IL.

Host Institution: 


\section{PLANTS OF}

This is a voucher specimen for

anticancer testing: YES NO

Fam.: Saurauriacear

Saurauria

Det. by:

Date:

Field characters:

Loc: $(3842)$

Lat.: $(3842)$ Long.:

Alt.:

Habitat: $(33</ 2)$

Loc. name(s):

Med. use(s):

Part(s) used:

General notes:

Hazard notes:

Collector(s):

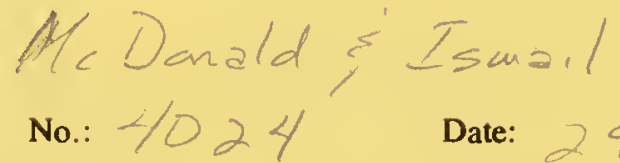

Sample plant part(s): $\angle F$

$\mathrm{NCI}$ code number(s):

$$
\text { Tw }
$$

$$
\angle A
$$

Collected under the auspices of:

U.S. National Cancer Institute, Bethesda, MD.

University of Illinois at Chicago, Chicago, IL.

Arnold Arboretum, Harvard University, Cambridge, MA

Field Museum of Natural History, Chicago, IL.

Host Institution: $B=, \angle, P$ 


\section{PLANTS OF}

This is a voucher specimen for

anticancer testing: YES _ NO

Fam.: $C / a c a c e a e$

Strom hosia

Det. by:

Date:

Field characters: $1 /$ ids losy tice

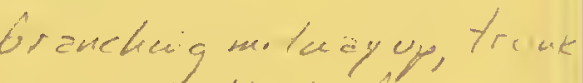
tur treal, $13 \mathrm{~cm}$ tian; bark rusty brown, smoothish

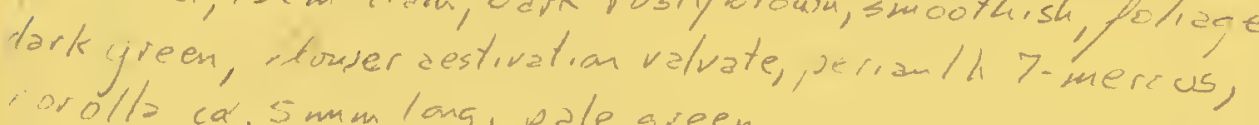

Loc: $(3,543)$

Lat.: $(35 / 2)$ Long.:

Alt.:

Habitat: $(2,42)$

Loc. name(s):

Med. use(s):

Part(s) used:

General notes:

Hazard notes:

Collector(s): H< Danald

No.: $4 / 025$

Date:

Sample plant part(s):

$\angle F$ 1442 


\section{PLANTS OF}

This is a voucher specimen for anticancer testing: YES NO

Fam.: Lythraceae

Lagerstroem ia

Det. by:

Date:

Field characters: $C 2$ nop

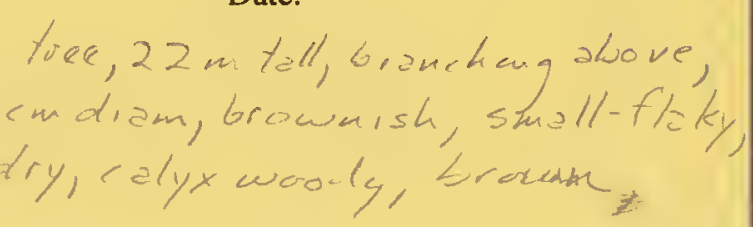

Loc.: $(334 / 2)$

Lat.: $(3842)$ Long.:

Alt:

Habitat: $(38 / 2)$

Loc. name(s):

Med. use(s):

Part(s) used:

General notes:

Hazard notes:

Collector(s):
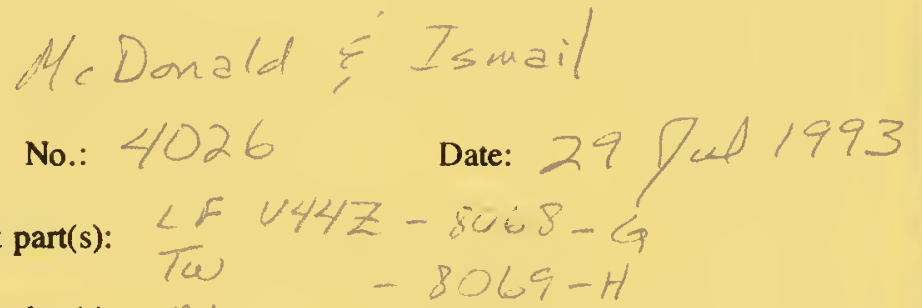

$\mathrm{NCI}$ code number(s): $\quad B, A$

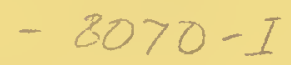

Collected under the auspices of:

U.S. National Cancer Institute, Bethesda, MD.

University of Illinois at Chicago, Chicago, IL.

Amold Arboretum, Harvard University, Cambridge, MA.

Field Museum of Natural History, Chicago, IL.

Host Institution: 


\section{PLANTS OF}

This is a voucher specimen for anticancer testing: YES NO

Fam.: Rosaceae

Prunus

Det. by:

Date:

Field characters:

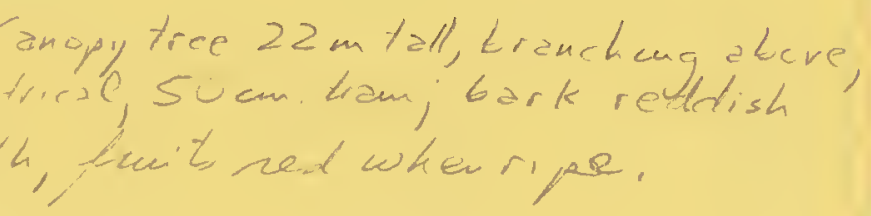

Loc: $(3842)$

Lat.: $(3842)$ Long.:

Alt:

Habitat: $(38 \% 2)$

Loc. name(s):

Med. use(s):

Part(s) used:

General notes:

Hazard notes:

Collector(s):

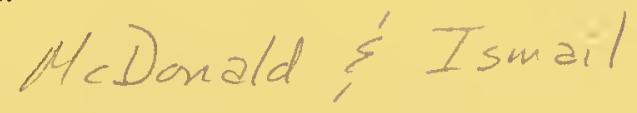

$$
\text { No.: } 4027 \text { Date: } 29 \text { Gul } 1993
$$

Sample plant part(s): LF $044 Z-8071-\sqrt{ }$

$\mathrm{NCI}$ code number(s):

Collected under the auspices of:

U.S. National Cancer Institute, Bethesda, MD.

University of Illinois at Chicago, Chicago, IL.

- Amold Arboretum, Harvard University, Cambridge, MA.

Field Museum of Natural History, Chicago, IL.

Host Institution: 


\section{PLANTS OF}

This is a voucher specimen for anticancer testing: YES $\_$NO

Fam.: Acan thoceae

Det. by:

Date:

Field characters:

Loc.: $(38 \% 2)$

Lat.: $(35 \% 2$ Long.:

Alt.:

Habitat: $(3342)$

Loc. name(s):

Med. use(s):

Part(s) used:

General notes:

Hazard notes:

Collector(s):
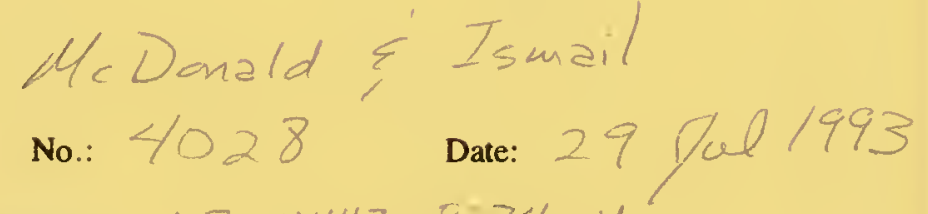

Sample plant part(s): $\angle F \quad 044 Z$ -

NCI code number(s): TLe

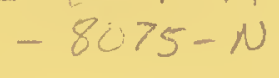

Collected under the auspices of:

U.S. National Cancer Institute, Bethesda, MD.

- University of Illinois at Chicago, Chicago, IL. Amold Arboretum, Harvard University, Cambridge, MA.

Field Museum of Natural History, Chicago, IL.

Host Institution: 


\section{PLANTS OF}

This is a voucher specimen for

anticancer testing: YES NO

Fam.: Lauracea e

Phoebe

Det. by:

Date:

Field characters:

Lat.: $(2,542)$ Long.:

Alt:

Habitat: $(33 / 2)$

Loc. name(s):

Med. use(s):

Part(s) used:

General notes:

Hazard notes:

Collector(s):

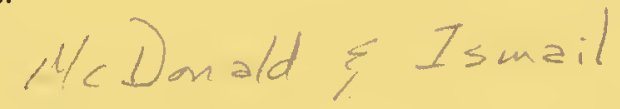

No.: 4029 Date: $29 \mathrm{lul} / 993$

Sample plant part(s): $\angle F \quad U 4 / Z-8076-0$

$\mathrm{NCI}$ code number(s):

Tw

Q1

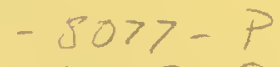

$-8078-Q$

Collected under the auspices of:

U.S. National Cancer Institute, Bethesda, MD.

University of Illinois at Chicago, Chicago, IL.

- Amold Arboretum, Harvard University, Cambridge, MA.

Field Museum of Natural History, Chicago, IL.

Host Institution: 


\section{PLANTS OF}

This is a voucher specimen for anticancer testing: YES NO

Fam.: Urticaceae

Det. by:

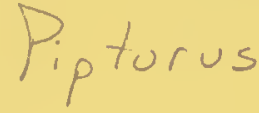

Field char

Date:

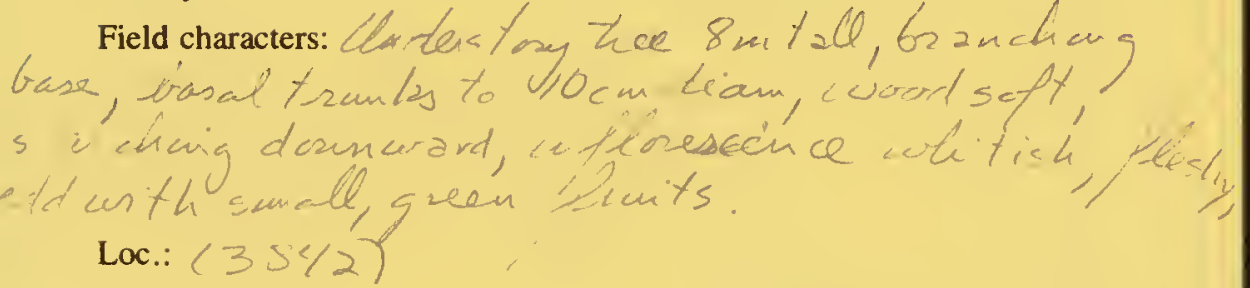

Loc.: $(3542)$

Lat.: $(\geqslant \rho 42)$ Long.:

Alt: :

Habitat: $(\geq \sqrt[J]{2})$

Loc. name(s):

Med. use(s):

Part(s) used:

General notes:

Hazard notes:

Collector(s):

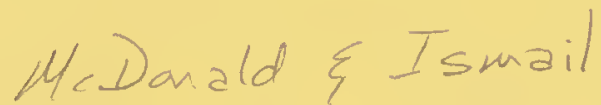

$$
\text { No.: } 4030 \quad \text { Date: } 29
$$

Sample plant part(s):

$\mathrm{NCI}$ code number(s):

$$
\text { TW }
$$

$B A$

$044 z$

$-8079-k$

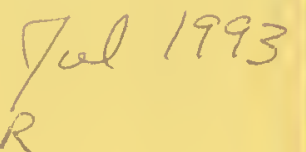

Collected under the auspices of:

U.S. National Cancer Institute, Bethesda, MD.

University of Illinois at Chicago, Chicago, IL.

Arnold Arboretum, Harvard University, Cambridge, MA.

Field Museum of Natural History, Chicago, IL.

Host Institution: 


\section{PLANTS OF}

This is a voucher specimen for anticancer testing: YES NO

Fam.: llubiaceae

$$
\text { Urophyllum }
$$

Det. by:

Date:

Field characters:

aps, Leents

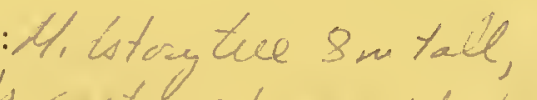

Loc.: $(38 \% 2)$

Lat.: $(33 / 2)$ Long.:

Alt.:

Habitat:

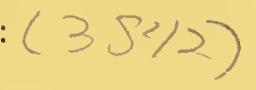

Loc. name(s):

Med. use(s):

Part(s) used:

General notes:

Hazard notes: Benk producos skun illargy
Collector(s): 4 CDonald $\xi$ Tsmail

$$
\text { No.: } 4031 \text { Date: } 29 \text { Tuel } 1993
$$

Sample plant part(s): $\angle F \quad U 4 / 4 Z-8082-U$

$\mathrm{NCI}$ code number(s):

$$
\text { Tw }
$$

$B A$

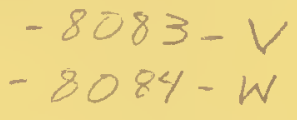

Collected under the auspices of:

U.S. National Cancer Institute, Bethesda, MD.

University of Illinois at Chicago, Chicago, IL.

Amold Arboretum, Harvard University, Cambridge, MA.

Field Museum of Natural History, Chicago, IL.

Host Institution: 


\section{PLANTS OF}

This is a voucher specimen for anticancer testing: YES NO

Fam.: J/u d ceae

$$
\text { ? Aphauantha }
$$

Det. by:

Date:

Field characters:

up, Zacule

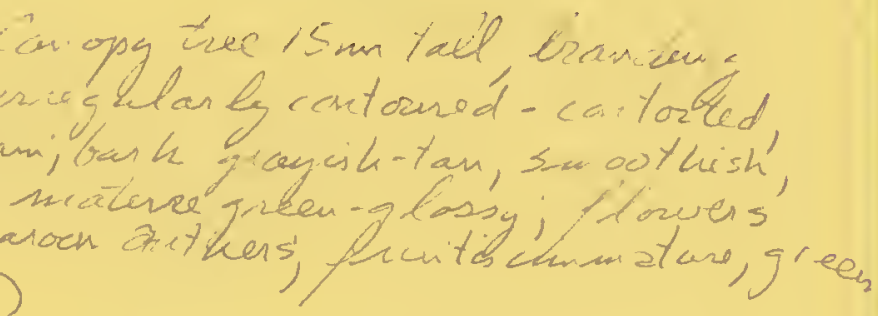

Lat.: $(3 P 42)$ Long.:

Alt :

Habitat: 3.542 )

Loc. name(s):

Med. use(s):

Part(s) used:

General notes:

Hazard notes:

Collector(s):

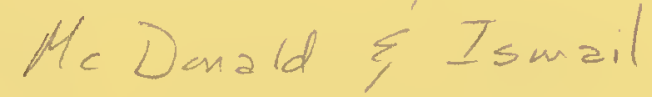

No.: 4032 Date: 29 /ul $/ 993$

Sample plant part(s): $\begin{aligned} & \angle F U 4 / 4 Z-8085-x \\ & T \omega J \quad-8086-Y\end{aligned}$

NCI code number(s): $\quad 3,4 \quad-8097-z$

Collected under the auspices of:

U.S. National Cancer Institute, Bethesda, MD.

University of Illinois at Chicago, Chicago, IL.

Amold Arboretum, Harvard University, Cambridge, MA.

Field Museum of Natural History, Chicago, IL.

Host Institution: 


\section{PLANTS OF}

This is a voucher specimen for

Fam.: Mor aceate<smiles>CCCCCCCCCC</smiles>

Det. by:

Field characters: anticancer testing: YES

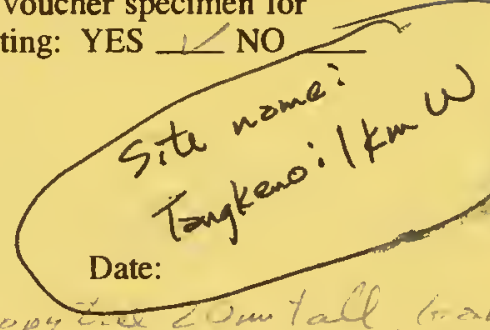

belcow, tranek Bmaliam

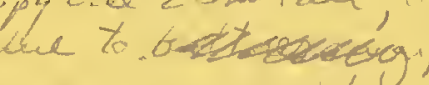

cenal toots, barkf gian, smooth, tan wa

Loc.

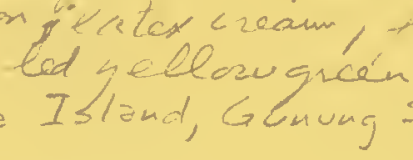

Tongkeno,

Lat.: $50 / 6^{\prime} \mathrm{S}$ Long.: $121^{\circ} 56^{\prime} \mathrm{E}^{-}$Alt: $700 \mathrm{~m}$ Habitat: Eslopes of ange, short forest savaumah,
cent assocvaths anclude Ficus, Hetrosileres Gardenia, Decasperma, Cratoxylan. Soils serpentine

Loc. name(s):

Med. use(s):

Part(s) used:

General notes:

Hazard notes:

Collector(s): M<Donald 5 Tsmeil

$$
\text { No.: } 4033 \text { Date: } 30 \text { Dul /993 }
$$

Sample plant part(s): $\angle F \quad 444 z^{7}-5089-0$

NCI code number(s): $B A \quad-8091-6$

Collected under the auspices of:

$$
\text { FR }-8092-H
$$

U.S. National Cancer Institute, Bethesda, MD.

University of Illinois at Chicago, Chicago, IL.

- Amold Arboretum, Harvard University, Cambridge, MA.

Field Museum of Natural History, Chicago, IL.

Host Institution: 


\section{PLANTS OF}

This is a voucher specimen for

anticancer testing: YES NO

Fam.: Si mar ouloaceae

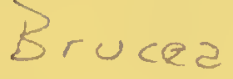

Det. by:

Date:

Field characters:

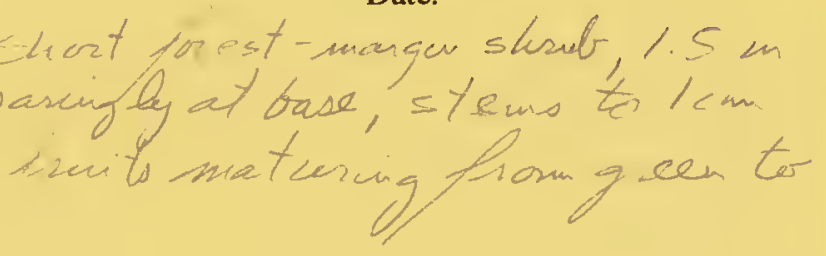

Loc.:

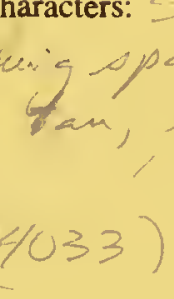

Lat: $(2 / 033)$ Long.:

Alt.:

Habitat: $4 / 033)$

Loc. name(s):

Med. use(s):

Part(s) used:

General notes:

Hazard notes:

Collector(s):

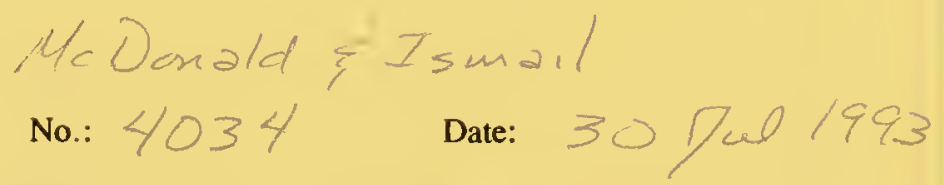

Sample plant part(s): $\quad \angle F \quad \cup 44 Z-$

$93-1$

$\mathrm{NCI}$ code number(s):

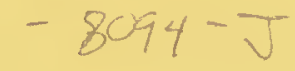

Collected under the auspices of:

U.S. National Cancer Institute, Bethesda, MD.

University of Illinois at Chicago, Chicago, IL.

Arnold Arboretum, Harvard University, Cambridge, MA.

Field Museum of Natural History, Chicago, IL.

Host Institution: 


\section{PLANTS OF}

This is a voucher specimen for anticancer testing: YES NO

Fam.: Nauleaceae

\section{Nauclea}

Det. by:

Date:

Field characters:

below $t_{2}$..

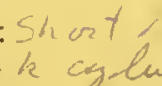

maing

t.e 7 m toll, Grancher. dat ot baxe; Leares surce Cet-condacrous,

Loc: $(4033)$

Lat.: $(4033)$ Long.:

Alt:

Habitat: $(4033)$

Loc. name(s):

Med. use(s):

Part(s) used:

General notes:

Hazard notes:

Collector(s):

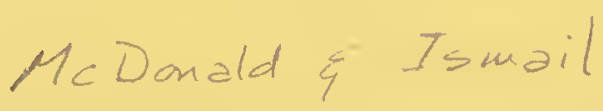

$$
\text { No.: } 4035 \text { Date: } 30 \text { Tel/993 }
$$

Sample plant part(s): $\angle F \quad U 44 Z-8095-K$

$\mathrm{NCl}$ code number(s):

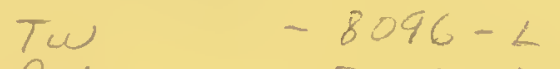

$$
-8097-M
$$

Collected under the auspices of:

U.S. National Cancer Institute, Bethesda, MD.

University of Illinois at Chicago, Chicago, IL.

- Arnold Arboretum, Harvard University, Cambridge, MA.

Field Museum of Natural History, Chicago, IL.

Host Institution: 


\section{PLANTS OF}

This is a voucher specimen for anticancer testing: YES $\_$NO

Fam.: Lauracea e

Actinodaphue

Det. by:

Date:

Field characters:

Loc.:

$$
(4033)
$$

Lat.: ( 4033$)$ Long.:

Alt.:

Habitat: $(4033)$

Loc. name(s):

Med. use(s):

Part(s) used:

General notes:

Hazard notes:

Collector(s):

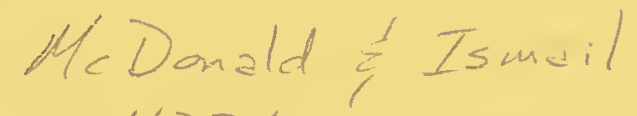

$$
\text { No.: } 4036 \quad \text { Date: }
$$

Sample plant part(s): $\angle F U 44 Z-8098-N$

$\mathrm{NCI}$ code number(s):

$$
\text { TW }
$$

$B A$

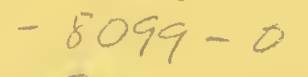

- $10100-P$

Collected under the auspices of:

U.S. National Cancer Institute, Bethesda, MD.

University of Illinois at Chicago, Chicago, IL.

- Amold Arboretum, Harvard University, Cambridge, MA.

Field Museum of Natural History, Chicago, IL.

Host Institution: 


\section{PLANTS OF}

This is a voucher specimen for anticancer testing: YES NO

Fam.: Clusiaceae Garcinia

Det. by:

Date:

Field characters:

in tall, trear cle en

colidricbl, baik nas

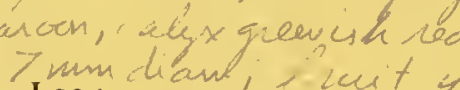

Loc.:

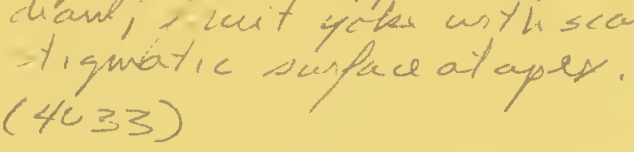

Lat.: $(4 / 0 \geq 3)$ Long.:

Alt.:

Habitat: 4033$)$

Loc. name(s):

Med. use(s):

Part(s) used:

General notes:

Hazard notes:

Collector(s):

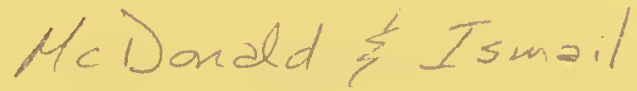

$$
\text { No.: } 4037 \text { Date: } 30
$$

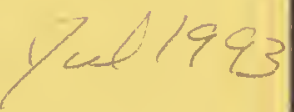

Sample plant part(s): $\angle F \quad U 44 Z=8101-Q$

NCI code number(s):

$$
\begin{array}{ll}
\text { Tw } & -8102-R \\
B A & -8103-S
\end{array}
$$

Collected under the auspices of:

U.S. National Cancer Institute, Bethesda, MD.

University of Illinois at Chicago, Chicago, IL.

- Arnold Arboretum, Harvard University, Cambridge, MA.

Field Museum of Natural History, Chicago, IL.

Host Institution: 


\section{PLANTS OF}

This is a voucher specimen for anticancer testing: YES NO

Fam.: Euphorbiaceae

\section{Bridelia}

Det. by:

Date:

Field characters: Short thee brancheng f com base, $5 \mathrm{~m}$ toll, busal stems to $5 \mathrm{~cm}$ diam, é Cuidrichel; tonk smooth, its an in terve, geeem.

Loc.: $(4033)$

Lat.: $(\angle / O \geqslant 3)$ Long.:

Alt: :

Habitat: $4 / 033$ )

Loc. name(s):

Med. use(s):

Part(s) used:

General notes:

Hazard notes:

Collector(s): Mc Danald E Ismail

$$
\text { No.: } 4038 \text { Date: } 30 \text { gel/993 }
$$

Sample plant part(s): $\angle F \quad U 44 Z-8104-T$

$\mathrm{NCI}$ code number(s):

$$
\text { Tw }
$$

BA

Collected under the auspices of:

U.S. National Cancer Institute, Bethesda, MD.

University of Illinois at Chicago, Chicago, IL.

- Amold Arboretum, Harvard University, Cambridge, MA.

Field Museum of Natural History, Chicago, IL.

Host Institution: 


\section{PLANTS OF}

This is a voucher specimen for

anticancer testing: YES NO

Fam.: Verbenaceae

Teijsmanniodendron

Det. by:

Date:

Field characters:

Loc. name(s):

Med. use(s):

Part(s) used:

General notes:

Hazard notes:

Collector(s):

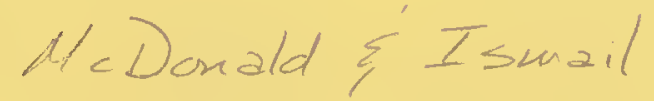

$$
\text { No.: } 4039 \text { Date: } 30 \text { pul } / 993
$$

Sample plant part(s): $\angle F \quad U 44 Z-8107-\omega$

$\mathrm{NCl}$ code number(s):

$$
\begin{aligned}
& \text { Ta }-8108-x \\
& \text { eA }
\end{aligned}
$$

Collected under the auspices of:

U.S. National Cancer Institute, Bethesda, MD.

University of Illinois at Chicago, Chicago, IL.

- Amold Arboretum, Harvard University, Cambridge, MA.

Field Museum of Natural History, Chicago, IL.

Host Institution: 


\section{PLANTS OF}

This is a voucher specimen for anticancer testing: YES NO

Fam.:

$$
\text { Cratoxylon }
$$

Det. by:

Date:

Field characters:

Loc.: bere wo tiekow,

$$
(4033)
$$

Lat:: $(4033)^{\text {Long.: }}$

Alt.:

Habitat: $(4033)$

Loc. name(s):

Med. use(s):

Part(s) used:

General notes:

Hazard notes:

Collector(s):
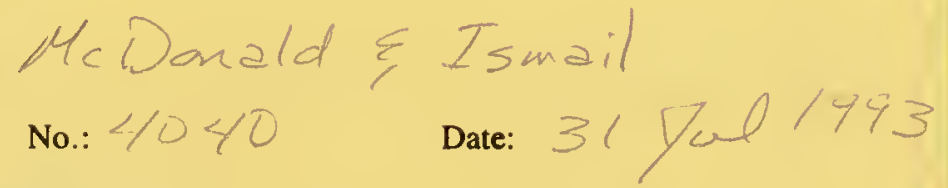

Sample plant part(s): $\quad \angle F \quad U 44 Z-8110-Z$

$\mathrm{NCl}$ code number(s):

$$
\text { Tw }
$$

BA

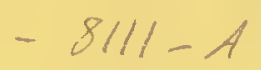

Collected under the auspices of:

U.S. National Cancer Institute, Bethesda, MD.

University of Illinois at Chicago, Chicago, IL.

- Arnold Arboretum, Harvard University, Cambridge, MA.

Field Museum of Natural History, Chicago, IL.

Host Institution: 


\section{PLANTS OF}

This is a voucher specimen for anticancer testing: YES

Fam.: Ku taceae ? Clausena

Det. by:

Date:

Field characters: coryinf,

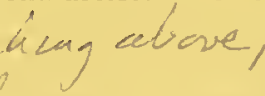

$$
(4033)
$$

Lat:: $(4033)$ Long.:

Alt.:

Habitat: 4033$)$

Loc. name(s):

Med. use(s):

Part(s) used:

General notes:

Hazard notes:

Collector(s):

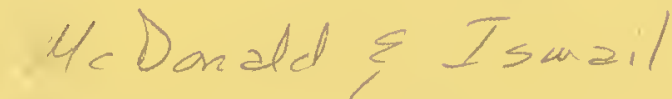

No.: $404 /$ Date: 31

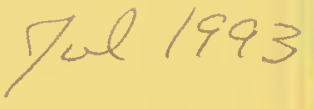

Sample plant part(s): $\quad \angle F \quad U 44 z-9 / 13-F$

NCI code number(s):

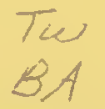

Collected under the auspices of:

U.S. National Cancer Institute, Bethesda, MD.

University of Illinois at Chicago, Chicago, IL.

Amold Arboretum, Harvard University, Cambridge, MA.

Field Museum of Natural History, Chicago, IL.

Host Institution: 


\section{PLANTS OF}

This is a voucher specimen for anticancer testing: YES NO

Fam.: $O / a$ caceae

Det. by:

Date:

Field characters:

$x_{1} 3$

Loc.:

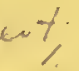

$(4033)$

Lat: $\left(4 / \mathrm{C}_{3} 3\right)$ Long.:

Alt :

Habitat: $(4 / 033)$

Loc. name(s):

Med. use(s):

Part(s) used:

General notes:

Hazard notes:

Collector(s):

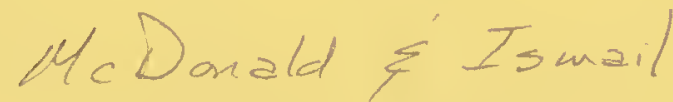

$$
\text { No.: } 4042 \text { Date: } 31 \mathrm{ful} / 993
$$

Sample plant part(s): $\angle F \quad U 4 / 2 Z-81 / C-I$

$\mathrm{NCI}$ code number(s):
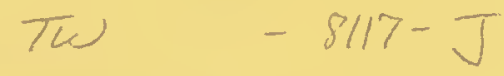

Collected under the auspices of:

U.S. National Cancer Institute, Bethesda, MD.

University of Illinois at Chicago, Chicago, IL.

- Amold Arboretum, Harvard University, Cambridge, MA.

Field Museum of Natural History, Chicago, IL.

Host Institution: 


\section{PLANTS OF}

This is a voucher specimen for anticancer testing: YES $\_$NO

Fam.: Urticaceae.

$$
\text { ? Boehweria }
$$

Det. by:

Date:

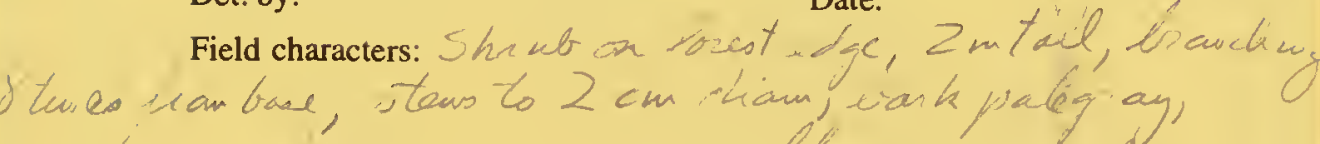
suroothish, trares conia crons, cuflosescence

$t$ urning whitish wsith age.
Loc.: $(4033)$

Lat.: $(4033)$ Long.:

Alt :

Habitat: $(4033)$

Loc. name(s):

Med. use(s):

Part(s) used:

General notes:

Hazard notes:

Collector(s): H<Darald E Ismail

$$
\text { No.: } 4043 \text { Date: } 319 \text { at } / 993
$$

Sample plant part(s): $\quad<F \quad U 44 Z-3 / 18-K$

NCI code number(s): The $-8 / 19-L$

Collected under the auspices of:

U.S. National Cancer Institute, Bethesda, MD.

University of Illinois at Chicago, Chicago, lL.

- Arnold Arboretum, Harvard University, Cambridge, MA.

Field Museum of Natural History, Chicago, IL.

Host Institution: $B$ 


\section{PLANTS OF}

This is a voucher specimen for anticancer testing: YES $\_$NO

Fam.: Orchidaceae

Varda

Det. by:

Date:

Field characters:

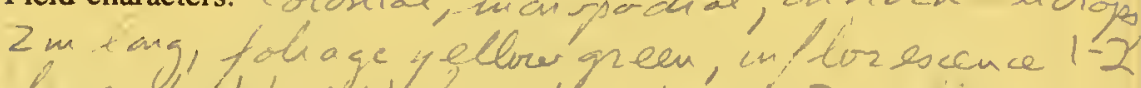

lower bud bright Leavender; ypolo 5 y t licus

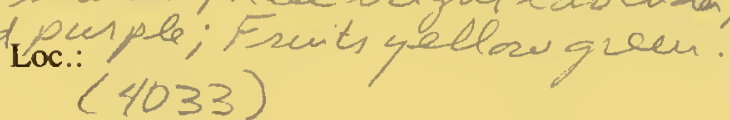

Lat::(4033) Long.:

Alt: :

Habitat: $4 / 033)$

Loc. name(s):

Med. use(s):

Part(s) used:

General notes:

Hazard notes:

Collector(s): McDanald $;$ Ismail

No.: 4044 Date: 31

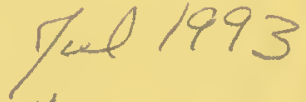

Sample plant part(s): $\angle F \quad \cup 44 Z-8120-M$

$\mathrm{NCI}$ code number(s):

$$
\text { Tw }
$$

Cat 7

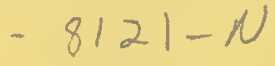

$-8122-0$

Collected under the auspices of: F $R-8123-P$

U.S. National Cancer Institute, Bethesda, MD.

University of Illinois at Chicago, Chicago, IL.

- Arnold Arboretum, Harvard University, Cambridge, MA.

Field Museum of Natural History, Chicago, IL.

Host Institution: $B$ 


\section{PLANTS OF}

This is a voucher specimen for anticancer testing: YES $\_$NO

Fam.: Urticaceae

Det. by:

Date:

Field characters: Shat thee an rock cutterop,

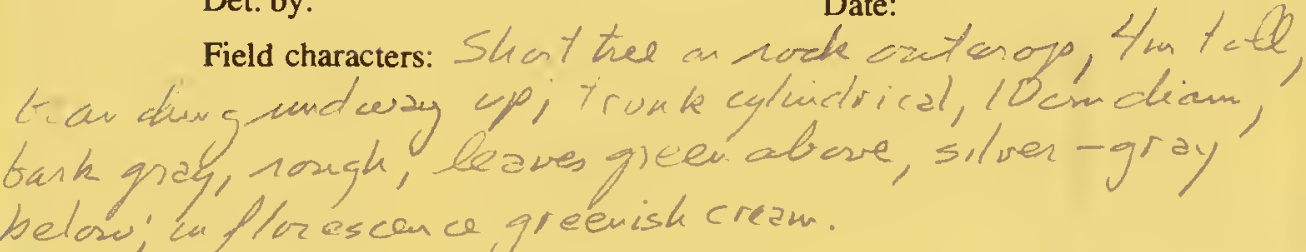

below; w for escence greenish crean.
Loc.: $(4033)$

Lat: $(4 / 033)$ Long.:

Alt.:

Habitat: $(4033)$

Loc. name(s):

Med. use(s):

Part(s) used:

General notes:

Hazard notes:

Collector(s): HcDaral $\{$ Ismail

$$
\text { No.: } 4045 \text { Date: } 317 \text { ul } / 993
$$

Sample plant part(s): $\angle F \quad U / 4 Z-8124-Q$

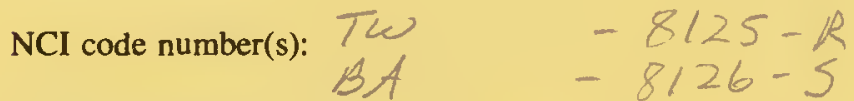

Collected under the auspices of:

U.S. National Cancer Institute, Bethesda, MD.

University of Illinois at Chicago, Chicago, IL.

- Arnold Arboretum, Harvard University, Cambridge, MA.

Field Museum of Natural History, Chicago, IL.

Host Institution: 


\section{PLANTS OF}

This is a voucher specimen for anticancer testing: YES NO

Fam.: Clusiaceae

Garcinia

Det. by:

Field characters: Cunopy thee un forest ravine, $12 \mathrm{~m}$
Yall, branchung above; trunk straight, cyluidsical, $13 \mathrm{~cm}$

Date: diam; banktan, rough; fruits unmature, green.

Loc.: $(4 / 033)$

Lat: $(4 / 033)$ Long.:

Alt.:

Habitat: $(4 / 033)$

Loc. name(s):

Med. use(s):

Part(s) used:

General notes:

Hazard notes:

Collector(s):

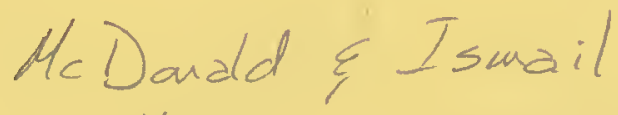

No.: 4048

Date: 31

Sample plant part(s): $\begin{aligned} & \text { LF U U } \\ & \text { TW }\end{aligned}$

NCI code number(s):

$B A$

$F=R$

Collected under the auspices of:

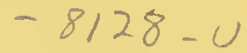

$-8129-v$

$-8130-w$

U.S. National Cancer Institute, Bethesda, MD.

University of Illinois at Chicago, Chicago, IL.

- Arnold Arboretum, Harvard University, Cambridge, MA. Field Museum of Natural History, Chicago, IL. Host Institution: 


\section{PLANTS OF}

This is a voucher specimen for anticancer testing: YES _ NO

Fam.: Heliaceae

? Didymoketan

Det. by:

Date:

Field characters: Ar terstong tha, 5 moll, brencleng above; runk cyludreal, 12 cudiam, bank jray, rough; Trwers on bud, thubulan, pale orange, suturess, reddish.

Loc.: $(4033)$

Lat.: ( $/ 033)$ Long.:

Alt.:

Habitat: $(4033)$

Loc. name(s):

Med. use(s):

Part(s) used:

General notes:

Hazard notes:

Collector(s):

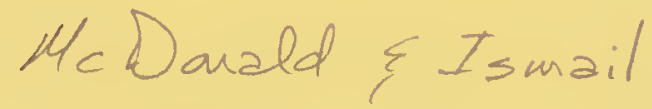

No.: 4049

Date: 3

Sample plant part(s): $L F \quad U 44 Z-8131-x$

$\mathrm{NCI}$ code number(s):

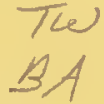
$-8132-y$$$
-8133-z
$$

Collected under the auspices of:

U.S. National Cancer Institute, Bethesda, MD.

University of Illinois at Chicago, Chicago, IL.

- Arnold Arboretum, Harvard University, Cambridge, MA. Field Museum of Natural History, Chicago, IL. Host Institution: $\mathrm{BC}$ 


\section{PLANTS OF}

This is a voucher specimen for anticancer testing: YES NO

Fam.: Lauraceae

Actiuat a phue

Det. by:
Field characters: 4, tstory tree $12 \mathrm{~m}$ tall, bi anching
ruak cylindrical, $20 \mathrm{~cm}$ diam; berk tan-graly,

Date:

trunk cylindrical, $20 \mathrm{~cm}$ diam; bark tan -graly, lough, t lower louds, pale yellow green, pelalsb, brown. fruits

immature, green

Loc.: $(4033)$

Lat: $(4033)$ Long.:

Alt :

Habitat: $(4 / 033)$

Loc. name(s):

Med. use(s):

Part(s) used:

General notes:

Hazard notes:

Collector(s):

$$
\begin{aligned}
& \text { McDonald } \xi \text { Tsmail } \\
& \text { No.: } 4050 \text { Date: } 31 \text { Yul } 1993
\end{aligned}
$$

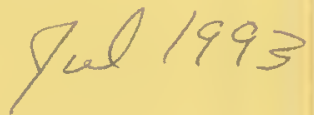

Sample plant part(s): $\quad \angle F \quad U 44 Z-8134-A$

NCI code number(s):

$$
\text { The }
$$

$B A$

$-8135-c$

Collected under the auspices of:

U.S. National Cancer Institute, Bethesda, MD.

University of Illinois at Chicago, Chicago, IL.

- Arnold Arboretum, Harvard University, Cambridge, MA.

Field Museum of Natural History, Chicago, IL.

Host Institution: 3 
PLANTS OF

Sulawesi

This is a voucher specimen for anticancer testing: YES

Fam.: Podocarpaceae Podocarpos

Bet. by:

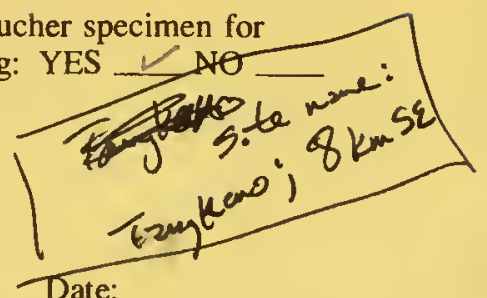

Date:

Field characters: History, yea 10 m tall, biandicyg midway up, lateral broaches perpendicular to trunk, in whorls of 3 , trunk "yea dire, Yea tiamjuark rusty brown, narrowly, vertically grooved, leaves dark green; seed green, aril fleshy, red at maturity.

Loci: Kabaena Island, Mt. Sangria Wi ta, ca. $8 \mathrm{~km} \mathrm{se}$ of Tangkeno.

Lat.: $5^{0} 19^{\prime} 5$ Long.: $121^{\circ} 58^{\prime} \mathrm{E}^{-}$Alt.: $1200 \mathrm{~m}$

Habitat: Forested NW slopes, canopy $10-20 \mathrm{~m}$ tall, ascraiates wiclude Eugenia, Psychotic ia, Podocaras Vernonia, Piflosporum, Melastana, etc. Soils serpent i Los. names):

Med. uses):

Parts) used:

General notes:

Hazard notes:

Collectors): He Donald \& Ismail

No.: 4051 Date: / Aug /993

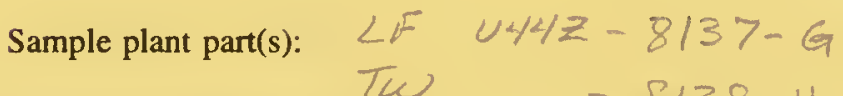

$12-S 138-H$

BA $\quad-8139-I$

Collected under the auspices of:

U.S. National Cancer Institute, Bethesda, MD.

University of Illinois at Chicago, Chicago, IL.

- Arnold Arboretum, Harvard University, Cambridge, MA. Field Museum of Natural History, Chicago, IL.

Host Institution: $E$ C 


\section{PLANTS OF}

This is a voucher specimen for anticancer testing: YES $\angle \mathrm{NO}$

Fam.: Araliaceae

Det. by:

Date:

Field characters: Canopy Yree $10 \mathrm{~m}$ Yall, broncheng sparingly akove; trunk cyliwdrical, $17 \mathrm{~cm}$ diam; bark gray, cleeply fissured, formung a diamond-latfice pattern, leaves Coriaceous, trower buds thak maroon, petals maroon, stamens pale yelluw,

fru,ts maroonish.

(4051)

Lat.:(4051) Long.:

Alt :

Habitat: $(4051)$

Loc. name(s):

Med. use(s):

Part(s) used:

General notes:

Hazard notes:

Collector(s):

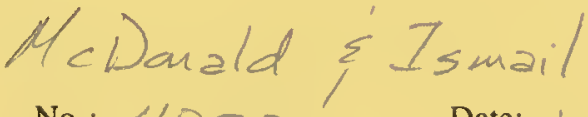

No.: 4052

Date: $8140-7$

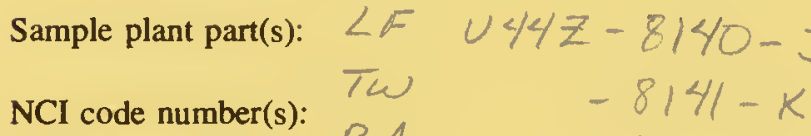

Collected under the auspices of:$$
-8142-L
$$

U.S. National Cancer Institute, Bethesda, MD.

University of Illinois at Chicago, Chicago, IL.

- Arnold Arboretum, Harvard University, Cambridge, MA. Field Museum of Natural History, Chicago, IL. Host Institution: $5 ;<|p|$ 


\section{PLANTS OF}

This is a voucher specimen for anticancer testing: YES NO

Fam.: Rubiaceae

Psychotria

Det. by:

Date:

Field characters: Co- tomenant, understory tree, $7 \mathrm{~m}$ tall, midway upj trwak crooked, cylindr,cal, $10 \mathrm{~cm}$ diam,

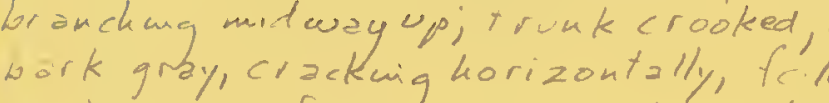
work gray, crackuighorizontally, friage dark green,
coriaceous, fruits orange to red turuigmaturatian

Loc.: $(4051)$

Lat.: $(4051)$ Long.:

Alt.:

Habitat: 4051 )

Loc. name(s):

Med. use(s):

Part(s) used:

General notes:

Hazard notes:

Collector(s):

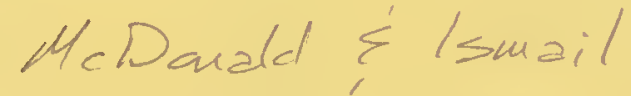

No.: 4053

Date:

Sample plant part(s)

$$
\begin{aligned}
& \angle F \\
& T W
\end{aligned}
$$

$\mathrm{NCI}$ code number(s):
$044 Z$

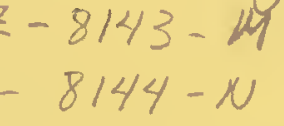

$-8145-0$

Collected under the auspices of:

U.S. National Cancer Institute, Bethesda, MD.

University of Illinois at Chicago, Chicago, IL.

- Arnold Arboretum, Harvard University, Cambridge, MA.

Field Museum of Natural History, Chicago, IL.

Host Institution: $B O-\angle 1 \%$ 


\section{PLANTS OF}

This is a voucher specimen for anticancer testing: YES $\_$NO

Fam.: Myrtaceae

Eugenia

Det. by:

Date: Field characters: (anopy tree $12 \mathrm{~m}$ tall, branching miduay,
upitrunk cylindical, $10 \mathrm{~cm}$ diam; bark gray, smooth, floral lise paleyellow, fruits creamygreen.

Loc.: $(4051)$

Lat.: $(4051)$ Long.:

Alt:

Habitat: 4051 )

Loc. name(s):

Med. use(s):

Part(s) used:

General notes:

Hazard notes:

Collector(s): $M \subset D$ arald $\&$ Tsmail

No.: 4054 Date:

Sample plant part(s): $\angle F \quad U 4 / 4 Z-8146-P$

NCI code number(s):

$$
\text { TW }
$$

$B A$

Collected under the auspices of:

U.S. National Cancer Institute, Bethesda, MD.

University of Illinois at Chicago, Chicago, IL.

Arnold Arboretum, Harvard University, Cambridge, MA.

Field Museum of Natural History, Chicago, IL.

Host Institution: 00 


\section{PLANTS OF Sulawes,}

This is a voucher specimen for anticancer testing: YES $\longleftarrow$ NO

Fam.: Celastraceae

Det. by:

Date:

Field characters: Camopy tree $13 \mathrm{~m}$ tall, branch ang above, trunk cylintrical-contoured, $30 \mathrm{~cm}$ diam; bisk brownish gray, tlaky, truits hard, yellowish and red. Loc: $(4051)$

Lat.:( $/ 051)$ Long.:

Alt.:

Habitat: $(1051)$

Loc. name(s):

Med. use(s):

Part(s) used:

General notes:

Hazard notes:

Collector(s):
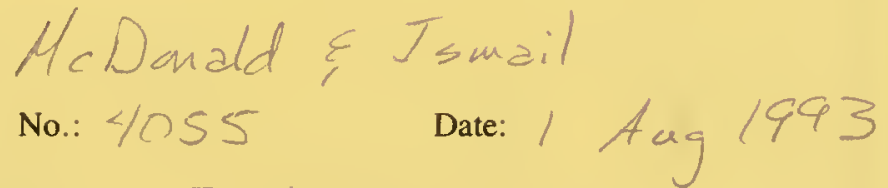

Sample plant part(s): $\angle F \quad U / 4 Z-8149-5$

NCI code number(s):

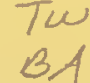

Collected under the auspices of:

$-3 / 50-7$

$-8151-0$

U.S. National Cancer Institute, Bethesda, MD.

University of Illinois at Chicago, Chicago, IL.

- Arnold Arboretum, Harvard University, Cambridge, MA.

Field Museum of Natural History, Chicago, IL.

Host Institution: $B . D, \angle I P$ ! 


\section{PLANTS OF}

This is a voucher specimen for anticancer testing: YES $\_$NO

Fam.: Asteraceae

Vernonia

Det. by:

Date:

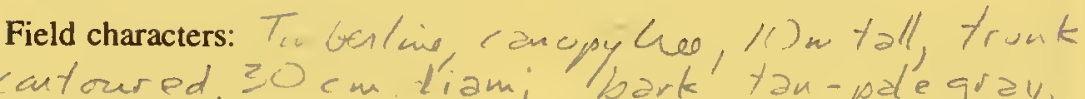
rioked cantoured, $30 \mathrm{~cm}$. L'iam; bark' tan-pal'e gray, lateral baanches wery crooked; la avescoriaceous Wood Lierd.

Loc.: $(4051)$

Lat.: $(4051)$ Long.:

Alt: :

Habitat: $(4051)$

Loc. name(s):

Med. use(s):

Part(s) used:

General notes:

Hazard notes:

Collector(s): He Douald \& Tsun il

$$
\text { No.: } 4056 \text { Date: I Aug } 1993
$$

Sample plant part(s): $L F U 44 Z-8152-V$

NCI code number(s):

$$
\text { Tw }-8153-\omega
$$

$B A$

$$
-8154-x
$$

Collected under the auspices of:

U.S. National Cancer Institute, Bethesda, MD.

University of Illinois at Chicago, Chicago, IL.

Arnold Arboretum, Harvard University, Cambridge, MA.

Field Museum of Natural History, Chicago, IL.

Host Institution: $E a, \quad, P \mid$ 


\section{PLANTS OF}

This is a voucher specimen for anticancer testing: YES — NO

Fam.: Daphuiphyllaceae
Daphniphyllum

Det. by:

Date:

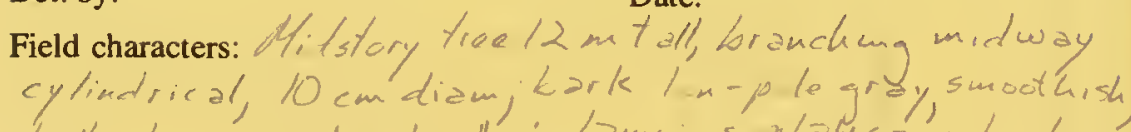

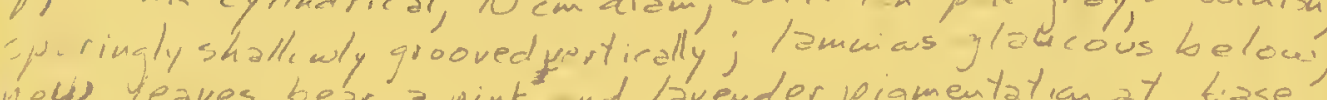
hew teaves bear a pink nd buender pigmentation at tase

of petiole; Lfowers staminate, arthers dark lavender

Lat.: $(4051)$ Long.:

Alt.:

Habitat $(2 / 051)$

Loc. name(s):

Med. use(s):

Part(s) used:

General notes:

Hazard notes:

Collector(s): 4 CDardel $\xi$ I smail

No.: 4057 Date: / Aug 1993

Sample plant part(s): $\angle F \quad U 44 z-8155-y$

NCI code number(s):

$$
\text { TW }
$$

$B A$

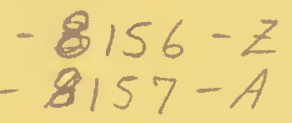

Collected under the auspices of:

U.S. National Cancer Institute, Bethesda, MD.

University of Illinois at Chicago, Chicago, IL.

- Amold Arboretum, Harvard University, Cambridge, MA.

Field Museum of Natural History, Chicago, IL.

Host Institution: $B \longrightarrow 2 i p$ 


\section{PLANTS OF}

This is a voucher specimen for anticancer testing: YES $\longleftarrow$ NO

Fam.: Lellelean Rutaceae

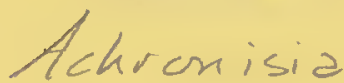

Det. by:

Date:

Field characters: Smiall tree Su tall, krancharg above and belou, triak cylintrical, $6 \mathrm{~cm} d i a m ; b a r k$ pale gray, smooth, imner bark tam, witlurescence pale
green, fowers cream; fruits immature, pale green
Loc.: Loc: $(4051)$

Lat.: $(4051)$ Long.:

Alt.:

Habitat:

$$
(4051)
$$

Loc. name(s):

Med. use(s):

Part(s) used:

General notes:

Hazard notes:

Collector(s): Me Danald $E$ Tsmail No.: $40 \cos ^{-8}$

Date:

Sample plant part(s): LF U44Z-815S-C

NCI code number(s):

$$
\text { The }
$$

$B A$

Collected under the auspices of:

- 8159-F

$-8160-G$

U.S. National Cancer Institute, Bethesda, MD.

University of Illinois at Chicago, Chicago, IL.

Arnold Arboretum, Harvard University, Cambridge, MA.

Field Museum of Natural History, Chicago, IL.

Host Institution: 
PLANTS OF

Sulawesi

This is a voucher specimen for anticancer testing: $\mathrm{YES} \perp \mathrm{NO}$

Fam.: $S a b, a c e a e$

Melisma

Site $\frac{\text { ranger o } 10}{10}$

Bet. by:

Date:

Field characters: Canopy tree $8 \mathrm{~m}$ tall, Luanchang above; truk cylurlrical, $14 \mathrm{~cm}$ diam; Lark gray, roughened by lenticel; leaves coriaceous, t lowers in bud, green; fruits immature, green and mottled brown,

Lac: Kabaena Island, Summit of Sangia Nita, ca. $10 \mathrm{~km}$ se of Tangkeno.

Lat.: $5^{\circ} 19^{\prime} 5$ Long.: $12158^{\prime} \dot{E}$ Alt.: $1500 \mathrm{~m}$

Habitat: Short scleropkyllous forest, sube/fin, Associates mande Melastoma, Actinotaphne, Melisma, Calamus, Daphniphyllum, atc. Soils Loc. names): serpentine

Med. uses):

Parts) used:

General notes:

Hazard notes:

Collectors): Mc Donald Isanail

No.: 4059 Date: / Aug 1993

Sample plant parts): $\angle F \quad U 44 Z-8 / C /-H$

NCI code numbers): $\begin{array}{ll}\text { Tu } & -8162-I \\ \text { BA } & -8163-T\end{array}$

Collected under the auspices of:

U.S. National Cancer Institute, Bethesda, MD.

University of Illinois at Chicago, Chicago, IL.

Arnold Arboretum, Harvard University, Cambridge, MA.

Field Museum of Natural History, Chicago, IL.

Host Institution: 


\section{PLANTS OF}

This is a voucher specimen for anticancer testing: YES

Fam.: Lauraceae

Det. by:

Date:

Field characters: Shrub $3 m$ tall, branchmg above and below basal stem $4 \mathrm{~cm}$ liam; ntw leaves =, lver, soff-rancscent,

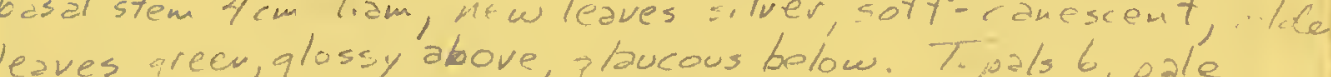
leaves grecr, glossy above, jlaucous below. $7 \mathrm{p} p \mathrm{pls} \mathrm{b}, \mathrm{pale}$ green, stamens 3. Fruits rotures black, ra. I cm diam, burn on a greencop ca. $8 \mathrm{~mm}$.tiam, 3 man tall.

$$
(4059)
$$

Lat: $(4 / 459)$ Long.: Alt.: $1500 \mathrm{~m}$

Habitat: ( 1059 )

Loc. name(s):

Med. use(s):

Part(s) used:

General notes:

Hazard notes:

Collector(s): Mc Douald E Isunail No.: 4060 Date: I A a 1993

Sample plant part(s): $\quad \angle F \quad U 44 Z-8164-K$

NCI code number(s):
TW $B, 4$

$$
\begin{aligned}
& -3165-2 \\
& -8166-M
\end{aligned}
$$

Collected under the auspices of:

U.S. National Cancer Institute, Bethesda, MD.

University of Illinois at Chicago, Chicago, IL.

Arnold Arboretum, Harvard University, Cambridge, MA.

Field Museum of Natural History, Chicago, IL.

Host Institution: $E=$ 


\section{PLANTS OF}

This is a voucher specimen for anticancer testing: YES NO

Fam.: Mor acea

$$
\text { Ficus }
$$

Det. by:

Date:

Field characters: Canopy tree $15 \mathrm{~m}$ tall, tranchmg above; trunk cylimdrual, / scm diam, burk gray, semewhat smooth, bearing cauliflorous branches. Figs raddish, spotted yollow (apple colors).

Loc.: $(3842)$

Lat: $(3842)$ Long.:

Alt:

Habitat: $(3842)$

Loc. name(s):

Med. use(s):

Part(s) used:

General notes:

Hazard notes:

Collector(s):

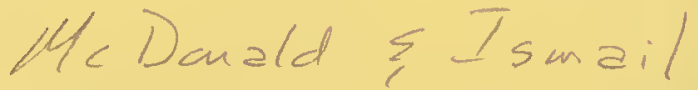

$$
\text { No.: } 4060-5 \text { Date: }
$$

Sample plant part(s): $\frac{\angle F}{T W} \cup 44 z-816>-W$

$\mathrm{NCI}$ code number(s): $B A$

Collected under the auspices of:

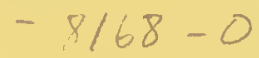

Fe

$-8169-P$

U.S. National Cancer Institute, Bethesda, MD.

University of Illinois at Chicago, Chicago, IL.

- Arnold Arboretum, Harvard University, Cambridge, MA.

Field Museum of Natural History, Chicago, IL.

Host Institution: 


\section{PLANTS OF}

This is a voucher specimen for

- anticancer testing: YES $\_$NO

Fam.: 52 potaceae

Det. by:

Date:

Field characters: Tice t m tall, biancheng above, trunk cylindrical, $30 \mathrm{~cm}$ hiam/berk grayish blown, omewihat green, flossy.
Loc.: $(38<2)$

Lat: $(38 \% \beta)$ Long.:

Alt:

Habitat:<smiles>C1C[As]C1</smiles>

Loc. name(s):

Med. use(s):

Part(s) used:

General notes:

Hazard notes:

Collector(s): HC Donald \& Ismail No.: $4 / 63 \quad$ Date: 6 Aug 1993

Sample plant part(s): LF U4/Z-8171-R

NCI code number(s):

$$
\text { Tw }
$$

$B A$

$-8172-5$

$-3173-1$

Collected under the auspices of:

U.S. National Cancer Institute, Bethesda, MD.

University of Illinois at Chicago, Chicago, IL.

- Arnold Arboretum, Harvard University, Cambridge, MA.

Field Museum of Natural History, Chicago, IL.

Host Institution: $B O,-\angle 1$ 


\section{PLANTS OF}

This is a voucher specimen for

anticancer testing: YES _ NO

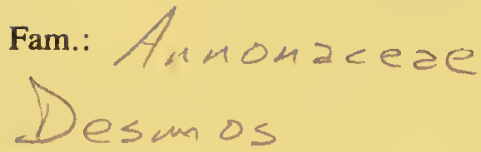

Det. by:

Date:

Field characters: Liana $7 \mathrm{~m}$ tall, much bramched; stems twining, to lum long, basal atere stems ca. 1/cm liam; bark dark grayor tepals un 2 series, broadly lanceolate, $2-5 \mathrm{~cm}$ long, $1-1.5$ cmwide, coriacecus, Loc.: yellow green.

$$
(3842)
$$

Lat.: $(38 / 2)$ Long.:

Alt.:

Habitat: $(3842)$

Loc. name(s):

Med. use(s):

Part(s) used:

General notes:

Hazard notes:

Collector(s): He Donald $\sum$ Tsmzil

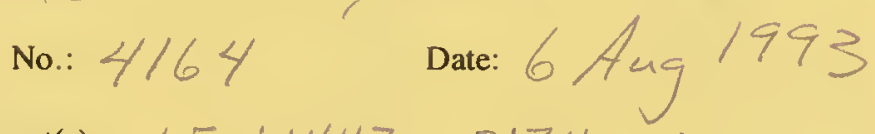

Sample plant part(s): $\angle F U 44 Z-8174-U$

NCI code number(s): Tw $-8175-\mathrm{V}$

Collected under the auspices of:

U.S. National Cancer Institute, Bethesda, MD.

University of lllinois at Chicago, Chicago, IL.

- Arnold Arboretum, Harvard University, Cambridge, MA.

Field Museum of Natural History, Chicago, IL. 


\section{PLANTS OF INDONESIA}

Sterculiaceae

Cat. \# 4004

Pterospermum celebicum Miq.

Det. By:

Locality:

Kabaena; Kabaena,

Mountain range between Tangkeno and Batu Sangia, 1-3 km W of Tangkeno.

$5^{\circ} 16^{\prime} \mathrm{S} 121^{\circ} 56^{\prime} \mathrm{E}$ Alt: $700-900 \mathrm{~m}$

Habitat:

Secondary forest around ladangs. Associates include Arenga, bamboos, Ficus, Trichospermum, Albizia.

Field Char:

Canopy tree $12 \mathrm{~m}$ tall, branching above; bole

straight, cylindrical, $20 \mathrm{~cm}$ diam.; bark tannish-gray, narrowly grooved; leaves green above, tannishpubescent below; calyx brown puberulent, cream inside, corolla white, stamens and style white, stigma pale-yellow green.

McDonald \& Ismail 4004

27 July 1993

\section{HERBARIUM BOGORIENSE HARVARD UNIVERSITY HERBARIA}

\section{PLANTS OF INDONESIA}

Fabaceae

Cat. \# 4005

\section{Albizia aff. corniculata}

Det. By: McDonald 1993

Locality:

Kabaena; Kabaena,

Mountain range between Tangkeno and Batu Sangia,

1-3 km W of Tangkeno.

$5^{\circ} 16^{\prime}$ S $121^{\circ} 56^{\prime} \mathrm{E}$ Alt: $700-900 \mathrm{~m}$

Habitat:

Secondary forest around ladangs. Associates include Arenga, bamboos, Ficus, Trichospermum, Albizia.

Field Char:

Short tree $7 \mathrm{~m}$ tall, branching above and below; bole cylindrical, $8 \mathrm{~cm}$. diam; bark grayish white; fruits green, mottled red.

McDonald \& Ismail 4005

27 July 1993

HERBARIUM BOGORIENSE HARVARD UNIVERSITY HERBARIA 


\section{PLANTS OF INDONESIA}

Tiliaceae

Cat. \# 4006

Trichospermum

Det. By: McDonald 1993

Locality:

Kabaena; Kabaena,

Mountain range between Tangkeno and Batu Sangia, 1-3 km W of Tangkeno.

$5^{\circ} 16^{\prime} \mathrm{S} 121^{\circ} 56^{\prime} \mathrm{E}$ Alt: $700-900 \mathrm{~m}$

Habitat:

Secondary forest around ladangs. Associates include Arenga, bamboos, Ficus, Trichospermum, Albizia.

Field Char:

Short tree $9 \mathrm{~m}$ tall, branching above and below; bole contoured-cylindrical, $30 \mathrm{~cm}$ diam.; bark pale gray, smooth; leaves scabrous; fruits dry, brown.

McDonald \& Ismail 4006

27 July 1993

\section{HERBARIUM BOGORIENSE HARVARD UNIVERSITY HERBARIA}

\section{PLANTS OF INDONESIA}

Moraceae

Cat. \# 4007

Ficus gul Ltb. \& K. Sch.

Det. By: McDonald 1993

Locality:

Kabaena; Kabaena,

Mountain range between Tangkeno and Batu Sangia,

$1-3 \mathrm{~km} \mathrm{~W}$ of Tangkeno.

$5^{\circ} 16^{\prime}$ 'S $121^{\circ} 56^{\prime} \mathrm{E}$ Alt: $700-900 \mathrm{~m}$

Habitat:

Secondary forest around ladangs. Associates include Arenga, bamboos, Ficus, Trichospermum, Albizia.

Field Char:

Canopy tree $20 \mathrm{~m}$ tall, branching midway up; trunk cylindrical, crooked, $20 \mathrm{~cm}$ diam; bark tannish-gray, smoothish; leaves scabrous below, fruits green.

McDonald \& Ismail 4007

27 July 1993

HERBARIUM BOGORIENSE HARVARD UNIVERSITY HERBARIA 


\section{PLANTS OF INDONESIA}

Acanthaceae

Cat. \# 4008

Hypoestes polythrysa Miq.

Det. By: McDonald 1993

Locality:

Kabaena; Kabaena,

Mountain range between Tangkeno and Batu Sangia, $1-3 \mathrm{~km} \mathrm{~W}$ of Tangkeno.

$5^{\circ} 16^{\prime} \mathrm{S} 121^{\circ} 56^{\prime} \mathrm{E}$ Alt: $700-900 \mathrm{~m}$

Habitat:

Secondary forest around ladangs. Associates include Arenga, bamboos, Ficus, Trichospermum, Albizia.

Field Char:

Understory shrub $2 \mathrm{mi}$ tall, branching prodigiously

from base; calyx green, tinged lavender, throat white, stamens lavender and exserted laterally to tube, style white.

McDonald \& Ismail 4008

27 July 1993

\section{HERBARIUM BOGORIENSE HARVARD UNIVERSITY HERBARIA}

\section{PLANTS OF INDONESIA}

Lecythidaceae

Cat. \# 4009

Barringtonia racemosa bl.

Det. By: McDonald 1993

Locality:

Kabaena; Kabaena,

Mountain range between Tangkeno and Batu Sangia,

$1-3 \mathrm{~km} \mathrm{~W}$ of Tangkeno.

$5^{\circ} 16^{\prime} \mathrm{S} 121^{\circ} 56^{\prime} \mathrm{E}$ Alt: $700-900 \mathrm{~m}$

Habitat:

Secondary forest around ladangs. Associates include Arenga, bamboos, Ficus, Trichospermum, Albizia.

Field Char:

Midstory tree $9 \mathrm{~m}$ tall, branching much midway up; bole straight, cylindrical, $15 \mathrm{~cm}$ diam.; bark tannishgray; inflorescence rachis dark pink, sepals 2 , gray and pink, petals 4 , very pale pink, stamens "hot" pink.

McDonald \& Ismail 4009

27 July 1993

HERBARIUM BOGORIENSE HARVARD UNIVERSITY HERBARIA 


\section{PLANTS OF INDONESIA}

Fabaceae

Cat. \# 4010

Cassia cf. timoriense $D C$.

Det. By: McDonald 1993

Locality:

Kabaena; Kabaena,

Mountain range between Tangkeno and Batu Sangia,

1-3 km W of Tangkeno.

$5^{\circ} 16^{\prime} \mathrm{S} 121^{\circ} 56^{\prime} \mathrm{E}$ Alt: $700-900 \mathrm{~m}$

Habitat:

Secondary forest around ladangs. Associates include Arenga, bamboos, Ficus, Trichospermum, Albizia.

Field Char:

Secondary tree $10 \mathrm{~m}$ tall, branching midway up; bole straight, cylindrical, $14 \mathrm{~cm}$ diam.; bark gray, rough, inner bark yellow; calyx green, petals 6, yellow, flower $1.5 \mathrm{~cm}$ wide, fertile stamens 6 , yellow, staminodes 3 , style and ovary green; fruits turning green to dark purple.

McDonald \& Ismail 4010

27 July 1993

\section{HERBARIUM BOGORIENSE HARVARD UNIVERSITY HERBARIA}

\section{PLANTS OF INDONESIA}

Cat. \# 4011

Tarrenoidea wallichii (Hook.f.) Tir.

Det. By: McDonald 1993

Locality:

Kabaena; Kabaena,

Batu Sangia peak, $5-7 \mathrm{~km}$ W of Tangkeno

$5^{\circ} 16^{\prime} 121^{\circ} 54^{\prime}$ Alt: $1000-1200$

Habitat:

Forest below cliffs, E slopes, mesophytic; Associates include Lithocarpus, Eugenia, Voacanga,

Lepisanthes, Celastraceae.

Field Char:

Midstory tree $13 \mathrm{~m}$ tall, branching above and below; bole straight, cylindrical, $13 \mathrm{~cm}$ diam.; bark cream with brown patches, ca. $1 \mathrm{~cm}$ thick, smooth; fruits immature, green.

McDonald \& Ismail 4011

27 July 1993

HERBARIUM BOGORIENSE HARVARD UNIVERSITY HERBARIA 


\section{PLANTS OF INDONESIA}

Sapindaceae

Cat. \# 4012

Guioa sp. nov.

Det. By: McDonald 1993

Locality:

Kabaena; Kabaena,

Batu Sangia peak, $5-7 \mathrm{~km}$ W of Tangkeno

$5^{\circ} 16^{\prime}$ 121 ${ }^{\circ} 54^{\prime}$ Alt: $1000-1200$

Habitat:

Forest below cliffs, E slopes, mesophytic; Associates include Lithocarpus, Eugenia, Voacanga,

Lepisanthes, Celastraceae.

Field Char:

Young canopy tree $14 \mathrm{~m}$ tall, branching above; trunk straight, cylindrical, $15 \mathrm{~cm}$ diam.; bark copperypubescent, calyx pale green, corolla white, ovary light green.

McDonald \& Ismail 4012

27 July 1993

HERBARIUM BOGORIENSE HARVARD UNIVERSITY HERBARIA

\section{PLANTS OF INDONESIA}

Fabaceae

Cat. \# 4013

\section{Ormosia macrodisca Baker}

Det. By: McDonald 1993

Locality:

Kabaena; Kabaena,

Batu Sangia peak, $5-7 \mathrm{~km}$ W of Tangkeno

$5^{\circ} 16^{\prime} 121^{\circ} 54^{\prime}$ Alt: $1000-1200$

Habitat:

Forest below cliffs, E slopes, mesophytic; Associates include Lithocarpus, Eugenia, Voacanga,

Lepisanthes, Celastraceae.

Field Char:

Canopy tree $17 \mathrm{~m}$ tall, branching above and below; bole cylindrical, $15 \mathrm{~cm}$ diam.; bark gray, smooth; foliage dark green; immature fruits light green.

McDonald \& Ismail 4013

27 July 1993

HERBARIUM BOGORIENSE HARVARD UNIVERSITY HERBARIA 


\section{PLANTS OF INDONESIA}

Myrtaceae

Cat. \# 4014

Eugenia sp.

Det. By: McDonald 1993

Locality:

Kabaena; Kabaena,

Batu Sangia peak, $5-7 \mathrm{~km}$ W of Tangkeno

$5^{\circ} 16^{\prime} 121^{\circ} 54^{\prime}$ Alt: $1000-1200$

Habitat:

Forest below cliffs, E slopes, mesophytic; Associates include Lithocarpus, Eugenia, Voacanga,

Lepisanthes, Celastraceae.

Field Char:

Midstory tree $12 \mathrm{~m}$ tall, branching above and below; bole straight, cylindrical, $10 \mathrm{~cm}$ diam.; bark rustybrown, thin, smoothish; foliage dark green; flowers cauliflorous on trunk; inflorescence and flower buds pale green, succulent, fragile.

McDonald \& Ismail 4014

27 July 1993

HERBARIUM BOGORIENSE

HARVARD UNIVERSITY HERBARIA

\section{PLANTS OF INDONESIA}

Moraceae

Cat. \# 4015

\section{Ficus asperiuscula}

Det. By: McDonald 1993

Locality:

Kabaena; Kabaena,

Batu Sangia peak, $5-7 \mathrm{~km}$ W of Tangkeno.

$5^{\circ} 16^{\prime}$ 'S $121^{\circ} 54^{\prime}$ E Alt: $1000-1200 \mathrm{~m}$

Habitat:

Forst below cliffs, E slopes, mesophytic. Associates include Lithocarpus, Eugenia, Voacanga,

Lepisanthes, Celastraceae.

Field Char:

Understory tree $6 \mathrm{~m}$ tall, branching midway up; bole cylindrical, $8 \mathrm{~cm}$ diam.; bark gray, smooth; leaves scabrous above and below; figs orange, bearing small, yellowish, foliaceous bracts.

McDonald \& Ismail 4015

28 July 1993

HERBARIUM BOGORIENSE HARVARD UNIVERSITY HERBARIA 


\section{PLANTS OF INDONESIA}

Cat. \# 4016

Ellatostachys verrucosum (Bl.) Radlk.

Det. By: McDonald 1993

Locality:

Kabaena; Kabaena,

Batu Sangia peak, 5-7 km W of Tangkeno.

$5^{\circ} 16$ 'S $121^{\circ} 54^{\prime} \mathrm{E}$ Alt: $1000-1200 \mathrm{~m}$

Habitat:

Forst below cliffs, E slopes, mesophytic. Associates include Lithocarpus, Eugenia, Voacanga,

Lepisanthes, Celastraceae.

Field Char:

Short canopy tree $13 \mathrm{~m}$ tall, branching midway up; bole somewhat contoured-cylindrical, $10 \mathrm{~cm}$ diam.; bark gray and covered with gray lichen; foliage dark green; fruits dry and brown, already dehisced.

McDonald \& Ismail 4016

28 July 1993

HERBARIUM BOGORIENSE

HARVARD UNIVERSITY HERBARIA

\section{PLANTS OF INDONESIA}

Lauraceae

Cat. \# 4017

Litsea sp.

Det. By: McDonald 1993

Locality:

Kabaena; Kabaena,

Batu Sangia peak, 5-7 km W of Tangkeno.

$5^{\circ} 16^{\prime} \mathrm{S} 121^{\circ} 54^{\prime} \mathrm{E}$ Alt: $1000-1200 \mathrm{~m}$

Habitat:

Forst below cliffs, E slopes, mesophytic. Associates include Lithocarpus, Eugenia, Voacanga,

Lepisanthes, Celastraceae.

Field Char:

Midstory tree $11 \mathrm{~m}$ tall, branching above; bole irregular, $50 \mathrm{~cm}$ diam.; bark brown with small, prominent, round lenticels; foliage dark green, laminas coriaceous; fruits abundant, green, speckled pale green.

McDonald \& Ismail 4017

28 July 1993

HERBARIUM BOGORIENSE HARVARD UNIVERSITY HERBARIA 


\section{PLANTS OF INDONESIA}

Moraceae

Cat. \# 4018

Ficus calophylla Bl.

Det. By: McDonald 1993

Locality:

Kabaena; Kabaena,

Batu Sangia peak, 5-7 km W of Tangkeno.

$5^{\circ} 16^{\prime} \mathrm{S} 121^{\circ} 54^{\prime} \mathrm{E}$ Alt: $1000-1200 \mathrm{~m}$

Habitat:

Forst below cliffs, E slopes, mesophytic. Associates include Lithocarpus, Eugenia, Voacanga,

Lepisanthes, Celastraceae.

Field Char:

Large tree at base of cliff, $10 \mathrm{~m}$ tall, branching from base and above; crown $20 \mathrm{~m}$ wide; bole cylindrical, $20 \mathrm{~cm}$ diam.; bark off-white, crusty-smooth; leaves succulent, coriaceous; figs cream.

McDonald \& Ismail 4018

28 July 1993

\section{HERBARIUM BOGORIENSE HARVARD UNIVERSITY HERBARIA}

\section{PLANTS OF INDONESIA}

Rubiaceae

Cat. \# 4019

Timonius belensis Merr. \& Perr.

Det. By: McDonald 1993

Locality:

Kabaena; Kabaena,

Batu Sangia peak, $5-7 \mathrm{~km} \mathrm{~W}$ of Tangkeno.

$5^{\circ} 16^{\prime} \mathrm{S} 121^{\circ} 54^{\prime} \mathrm{E}$ Alt: $1000-1200 \mathrm{~m}$

Habitat:

Forst below cliffs, E slopes, mesophytic. Associates include Lithocarpus, Eugenia, Voacanga, Lepisanthes, Celastraceae.

Field Char:

Shrubby tree $5 \mathrm{~m}$ tall, branching from base and above; basal stems to $8 \mathrm{~cm}$ diam., cylindrical; bark dark brownish-gray, rough, narrowly grooved; corolla yellow green, limb segments 7, reflexed; fruits immature, green.

McDonald \& Ismail 4019

28 July 1993

HERBARIUM BOGORIENSE HARVARD UNIVERSITY HERBARIA 


\section{PLANTS OF INDONESIA}

Celastraceae

Cat. \# 4020

Euonymus javanicus $B l$.

Det. By: McDonald 1993

Locality:

Kabaena; Kabaena,

Batu Sangia peak, 5-7 km W of Tangkeno.

$5^{\circ} 16^{\prime} \mathrm{S} 121^{\circ} 54^{\prime} \mathrm{E}$ Alt: $1000-1200 \mathrm{~m}$

Habitat:

Forst below cliffs, E slopes, mesophytic. Associates include Lithocarpus, Eugenia, Voacanga,

Lepisanthes, Celastraceae.

Field Char:

Midstory tree $11 \mathrm{~m}$ tall, branching midway up; bole straight, cylindrical, $10 \mathrm{~cm}$ diam.; bark dark brown, narrowly grooved, new stem growth green; flowers pale green; fruit dull pink, valves 5; seed black with bright orange aril.

McDonald \& Ismail 4020

28 July 1993

HERBARIUM BOGORIENSE

HARVARD UNIVERSITY HERBARIA

\section{PLANTS OF INDONESIA}

Fabaceae

Cat. \# 4021

Fabaceae Strongylodon lucidus (G.Forst.) seem.

Det. By: MeDonald 1993- det. J. Barham (k) 4/1998

Locality:

Kabaena; Kabaena,

Gunung Sabampolulu, $1 \mathrm{~km}$ S-SE of Tangkeno

5०16'S $121^{\circ} 56^{\prime} \mathrm{E}$ Alt: $700-900 \mathrm{~m}$

Habitat:

W slopes of range, mixed ecotone of grasslands and short forest; arborescent associates include Grevillea, Helicia, Gardenia, Vaccinium, Eugenia; soils serpentine.

Field Char:

Liana climbing to $8 \mathrm{~m}$; basal stems to $3 \mathrm{~cm}$; bark pale tan-gray, rough; leaves dark green; fruits immature, green, spongy.

McDonald \& Ismail 4021

29 July 1993

HERBARIUM BOGORIENSE HARVARD UNIVERSITY HERBARIA 


\section{PLANTS OF INDONESIA}

Myristicaceae

Cat. \# 4022

Myristica cf. simiarum A. DC.

Det. By: McDonald 1993

Locality:

Kabaena; Kabaena,

Gunung Sabampolulu, $1 \mathrm{~km}$ S-SE of Tangkeno

$5^{\circ} 16^{\prime} \mathrm{S} 121^{\circ} 56^{\prime} \mathrm{E}$ Alt: $700-900 \mathrm{~m}$

Habitat:

W slopes of range, mixed ecotone of grasslands and short forest; arborescent associates include Grevillea, Helicia, Gardenia, Vaccinium, Eugenia; soils serpentine.

Field Char:

Canopy tree $15 \mathrm{~m}$ tall, branching midway up, lateral branches whorled, perpendicular to bole; bole $30 \mathrm{~cm}$ diam.; bark grayish, rough, large-flaky; fruits solitary, terminal, pendent, orange.

McDonald \& Ismail 4022

29 July 1993

\section{HERBARIUM BOGORIENSE HARVARD UNIVERSITY HERBARIA}

\section{PLANTS OF INDONESIA}

Rubiaceae

Cat. \# 4023

Psychotria sp.

Det. By: McDonald 1993

Locality:

Kabaena; Kabaena,

Gunung Sabampolulu, $1 \mathrm{~km}$ S-SE of Tangkeno

$5^{\circ} 16^{\prime} \mathrm{S} 121^{\circ} 56^{\prime} \mathrm{E}$ Alt: $700-900 \mathrm{~m}$

Habitat:

W slopes of range, mixed ecotone of grasslands and short forest; arborescent associates include Grevillea, Helicia, Gardenia, Vaccinium, Eugenia; soils serpentine.

Field Char:

Small, marginal-forest tree, $5 \mathrm{~m}$ tall, branching above, crown round; bole cylindrical, $6 \mathrm{~cm}$ diam.; bark pale gray, smooth; fruits immature, pale green.

McDonald \& Ismail 4023

29 July 1993

HERBARIUM BOGORIENSE HARVARD UNIVERSITYY HERBARIA 


\section{PLANTS OF INDONESIA}

Cat. \# 4024

Saurauria aff. nudiflora $D C$.

Det. By: McDonald 1993

Locality:

Kabaena; Kabaena,

Gunung Sabampolulu, $1 \mathrm{~km}$ S-SE of Tangkeno

$5^{\circ} 16^{\prime} \mathrm{S} 121^{\circ} 56^{\prime} \mathrm{E}$ Alt: $700-900 \mathrm{~m}$

Habitat:

W slopes of range, mixed ecotone of grasslands and short forest; arborescent associates include Grevillea, Helicia, Gardenia, Vaccinium, Eugenia; soils serpentine.

Field Char:

Shrub in ravine, 10 stems branching from base, these to $4 \mathrm{~cm}$ diam.; bark gray, smoothish; stems and leaves almost devoid of hairs; calyx white, corolla campanulate-urceolate and white, stamens yellow, styles 4 , white.

McDonald \& Ismail 4024

29 July 1993

HERBARIUM BOGORIENSE

HARVARD UNIVERSITY HERBARIA

\section{PLANTS OF INDONESIA}

Cat. \# 4025

Anacalosia frutescens (Bl.) Bl.

Det. By: McDonald 1993

Locality:

Kabaena; Kabaena,

Gunung Sabampolulu, $1 \mathrm{~km} \mathrm{S-SE} \mathrm{of} \mathrm{Tangkeno}$

$5^{\circ} 16^{\prime} \mathrm{S} 121^{\circ} 56^{\prime} \mathrm{E}$ Alt: $700-900 \mathrm{~m}$

Habitat:

W slopes of range, mixed ecotone of grasslands and short forest; arborescent associates include Grevillea, Helicia, Gardenia, Vaccinium, Eugenia; soils serpentine.

Field Char:

Midstory tree, branching midway up; bole cylindrical, $13 \mathrm{~cm}$ diam.; bark rusty brown, smoothish; foliage dark green; flower aestivation valvate, perianth 7 -merous, corolla ca. $5 \mathrm{~mm}$ long, pale green. 


\section{PLANTS OF INDONESIA}

Lythraceae

Cat. \# 4026

Lagerstroemia speciosa Miq.

Det. By: McDonald 1993

Locality:

Kabaena; Kabaena,

Gunung Sabampolulu, $1 \mathrm{~km}$ S-SE of Tangkeno

$5^{\circ} 16^{\prime} \mathrm{S} 121^{\circ} 56^{\prime} \mathrm{E}$ Alt: $700-900 \mathrm{~m}$

Habitat:

W slopes of range, mixed ecotone of grasslands and short forest; arborescent associates include Grevillea, Helicia, Gardenia, Vaccinium, Eugenia; soils serpentine.

Field Char:

Canopy tree $22 \mathrm{~m}$ tall, branching above; bole much contoured, $70 \mathrm{~cm}$ diam.; bark brownish, small flaky; leaves coriaceous; fruits dry, calyx woody, brown.

McDonald \& Ismail 4026

29 July 1993

\section{HERBARIUM BOGORIENSE HARVARD UNIVERSITY HERBARIA}

\section{PLANTS OF INDONESIA}

Rosaceae

Cat. \# 4027

Prunus arborea Bl. Kalk.

Det. By: McDonald 1993

Locality:

Kabaena; Kabaena,

Gunung Sabampolulu, $1 \mathrm{~km}$ S-SE of Tangkeno

$5^{\circ} 16^{\prime} \mathrm{S} 121^{\circ} 56^{\prime} \mathrm{E}$ Alt: $700-900 \mathrm{~m}$

Habitat:

W slopes of range, mixed ecotone of grasslands and short forest; arborescent associates include Grevillea, Helicia, Gardenia, Vaccinium, Eugenia; soils serpentine.

Field Char:

Canopy tree $22 \mathrm{~m}$ tall, branching above; bole straight, cylindrical, $50 \mathrm{~cm}$ diam.; bark reddish tan, somewhat smooth; fruits red when ripe.

McDonald \& Ismail 4027

29 July 1993

HERBARIUM BOGORIENSE HARVARD UNIVERSITY HERBARIA 


\section{PLANTS OF INDONESIA}

Acanthaceae

Cat. \# 4028

\section{Acanthaceae}

Det. By: McDonald 1993

Locality:

Kabaena; Kabaena,

Gunung Sabampolulu, $1 \mathrm{~km} \mathrm{S-SE} \mathrm{of} \mathrm{Tangkeno}$

$5^{\circ} 16^{\prime} \mathrm{S} 121^{\circ} 56^{\prime} \mathrm{E}$ Alt: $700-900 \mathrm{~m}$

Habitat:

W slopes of range, mixed ecotone of grasslands and short forest; arborescent associates include Grevillea, Helicia, Gardenia, Vaccinium, Eugenia; soils serpentine.

Field Char:

Understory shrub branching from base, stems brittle, 1-2 cm diam.; leaves terminal; bracts pale green,

corolla white, speckled yellow and brown inside tube, stamens bent and exserted laterally.

McDonald \& Ismail 4028

29 July 1993

HERBARIUM BOGORIENSE

HARVARD UNIVERSITY HERBARIA

\section{PLANTS OF INDONESIA}

Lauraceae

Cat. \# 4029

Alseodaphne sp.

Det. By: McDonald 1993

Locality:

Kabaena; Kabaena,

Gunung Sabampolulu, $1 \mathrm{~km}$ S-SE of Tangkeno

$5^{\circ} 16^{\prime} \mathrm{S} 121^{\circ} 56^{\prime} \mathrm{E}$ Alt: $700-900 \mathrm{~m}$

Habitat:

W slopes of range, mixed ecotone of grasslands and short forest; arborescent associates include Grevillea, Helicia, Gardenia, Vaccinium, Eugenia; soils serpentine.

Field Char:

Canopy tree $20 \mathrm{~m}$ tall, branching midway up; bole cylindrical, $30 \mathrm{~cm}$ diam.; bark grayish brown, narrowly ridged; calyx 3-merous, pale green, stamens 6 , anthers brown, ovary pale green.

McDonald \& Ismail 4029

29 July 1993

HERBARIUM BOGORIENSE HARVARD UNIVERSITY HERBARIA 


\title{
PLANTS OF INDONESIA
}

Cat. \# 4030

Villebrunea capitellata (Bl.) Bl.

Det. By: McDonald 1993

Locality:

Kabaena; Kabaena,

Gunung Sabampolulu, $1 \mathrm{~km}$ S-SE of Tangkeno

$5^{\circ} 16^{\prime} \mathrm{S} 121^{\circ} 56^{\prime} \mathrm{E}$ Alt: $700-900 \mathrm{~m}$

Habitat:

W slopes of range, mixed ecotone of grasslands and short forest; arborescent associates include Grevillea, Helicia, Gardenia, Vaccinium, Eugenia; soils serpentine.

Field Char:

Understory tree $8 \mathrm{~m}$ tall, branching from base; basal trunks to $10 \mathrm{~cm}$ diam., wood soft, stems arching downward; inflorescence whitish, fleshy, embedded with small fruits.

McDonald \& Ismail 4030

29 July 1993

\author{
HERBARIUM BOGORIENSE \\ HARVARD UNIVERSITY HERBARIA
}

\section{PLANTS OF INDONESIA}

Loganiaceae

Cat. \# 4031

Geniostoma rupestre Forst.

Det. By: McDonald 1993

Locality:

Kabaena; Kabaena,

Gunung Sabampolulu, $1 \mathrm{~km} \mathrm{S-SE} \mathrm{of} \mathrm{Tangkeno}$

$5^{\circ} 16^{\prime} \mathrm{S} 121^{\circ} 56^{\prime} \mathrm{E}$ Alt: $700-900 \mathrm{~m}$

Habitat:

W slopes of range, mixed ecotone of grasslands and short forest; arborescent associates include Grevillea, Helicia, Gardenia, Vaccinium, Eugenia; soils serpentine.

Field Char:

Midstory tree $8 \mathrm{~m}$ tall, branching midway up; trunk contoured-grooved, $10 \mathrm{~cm}$ diam.; bark gray, smooth; foliage coriaceous; fruits pale green.

McDonald \& Ismail 4031

29 July 1993

HERBARIUM BOGORIENSE HARVARD UNIVERSITY HERBARIA 


\section{PLANTS OF INDONESIA}

Ulmaceae

Cat. \# 4032

Aphananthe cuspidata (Bl.) Planch.

Det. By: McDonald 1993

Locality:

Kabaena; Kabaena,

Gunung Sabampolulu, $1 \mathrm{~km}$ S-SE of Tangkeno

$5^{\circ} 16^{\prime} \mathrm{S} 121^{\circ} 56^{\prime} \mathrm{E}$ Alt: $700-900 \mathrm{~m}$

Habitat:

W slopes of range, mixed ecotone of grasslands and short forest; arborescent associates include Grevillea, Helicia, Gardenia, Vaccinium, Eugenia; soils serpentine.

Field Char:

Canopy tree $15 \mathrm{~m}$ tall, branching midway up; bole irregularly contoured-contorted, buttressed, $70 \mathrm{~cm}$ diam.; bark grayish-tan, smoothish; new leaves reddish, mature leaves reddish-glossy; flowers pale green with maroon anthers; fruits immature, green.

McDonald \& Ismail 4032

29 July 1993

\section{HERBARIUM BOGORIENSE HARVARD UNIVERSITY HERBARIA}

\section{PLANTS OF INDONESIA}

Moraceae

Cat. \# 4033

Ficus depressa Bl.

Det. By: McDonald 1993

Locality:

Kabaena; Kabaena,

Gunung Sabampolulu, $1 \mathrm{~km} \mathrm{~W}$ of Tangkeno.

$5^{\circ} 16^{\prime} 121^{\circ} 56^{\prime}$ Alt: $700 \mathrm{~m}$

Habitat:

E slopes of range, short forest savannah.

Arborescent associates include Ficus, Metrosideros,

Gardenia, Decasperma, Cratoxyla. Soils serpentine.

Field Char:

Occasional canopy tree $20 \mathrm{~m}$ tall, branching above and below; trunk $3 \mathrm{~m}$ diam. due to thickening aerial roots; bark gray, smooth; lamina light green with yellow venation; latex white; figs abundant, light green, speckled light green.

McDonald \& Ismail 4033

30 July 1993

HERBARIUM BOGORIENSE HARVARD UNIVERSITY HERBARIA 


\section{PLANTS OF INDONESIA}

Simaroubaceae

Cat. \# 4034

Brucea javanica (L.) Merr.

Det. By: McDonald 1993

Locality:

Kabaena; Kabaena,

Gunung Sabampolulu, $1 \mathrm{~km} \mathrm{~W}$ of Tangkeno.

$5^{\circ} 16^{\prime} 121^{\circ} 56^{\prime}$ Alt: $700 \mathrm{~m}$

Habitat:

E slopes of range, short forest savannah.

Arborescent associates include Ficus, Metrosideros,

Gardenia, Decasperma, Cratoxyla. Soils serpentine.

Field Char:

Short forest-margin shrub, $1.5 \mathrm{~m}$ tall, branching

sparingly at base; stems to $1 \mathrm{~cm}$ diam.; bark pale tan; fruits maturing from green to black.

McDonald \& Ismail 4034

30 July 1993

\section{HERBARIUM BOGORIENSE \\ HARVARD UNIVERSITY HERBARIA}

\section{PLANTS OF INDONESIA}

Rubiaceae

Cat. \# 4035

\section{Nauclea unicapitulifera Ridsd.}

Det. By: McDonald 1993

Locality:

Kabaena; Kabaena,

Gunung Sabampolulu, $1 \mathrm{~km} \mathrm{~W}$ of Tangkeno.

$5^{\circ} 16^{\prime} 121^{\circ} 56^{\prime}$ Alt: $700 \mathrm{~m}$

Habitat:

E slopes of range, short forest savannah.

Arborescent associates include Ficus, Metrosideros,

Gardenia, Decasperma, Cratoxyla. Soils serpentine.

Field Char:

Short forst-margin tree $7 \mathrm{~m}$ tall, brancing above and below; trunk cylindrical-angular; bark reddish-tan,

flaking somewhat at base; leaves succulent-

coriaceous, green with yellow-green venation;

inflorescence dry, brown.

McDonald \& Ismail 405

30 July 1993

HERBARIUM BOGORIENSE HARVARD UNIVERSITY HERBARIA 


\section{PLANTS OF INDONESIA}

Cat. \# 4036

Machilus sp.

Det. By: McDonald 1993

Locality:

Kabaena; Kabaena,

Gunung Sabampolulu, $1 \mathrm{~km}$ W of Tangkeno.

$5^{\circ} 16^{\prime} 121^{\circ} 56^{\prime}$ Alt: $700 \mathrm{~m}$

Habitat:

E slopes of range, short forest savannah.

Arborescent associates include Ficus, Metrosideros, Gardenia, Decasperma, Cratoxyla. Soils serpentine.

Field Char:

Canopy tree in forest islands of grassland, $12 \mathrm{~m}$ tall, branching above and below; bole $35 \mathrm{~cm}$ diam; bark pale gray, rough; foliage light green; inflorescence branching, yellow green; tepals 6, pale cream-green, greenish at base, anthers yoke.

McDonald \& Ismail 4036

30 July 1993

\section{HERBARIUM BOGORIENSE HARVARD UNIVERSITY HERBARIA}

\section{PLANTS OF INDONESIA}

Clusiaceae

Cat. \# 4037

Garcinia sp.

Det. By: McDonald 1993

Locality:

Kabaena; Kabaena,

Gunung Sabampolulu, $1 \mathrm{~km}$ W of Tangkeno.

$5^{\circ} 16^{\prime} 121^{\circ} 56^{\prime}$ Alt: $700 \mathrm{~m}$

Habitat:

E slopes of range, short forest savannah.

Arborescent associates include Ficus, Metrosideros,

Gardenia, Decasperma, Cratoxyla. Soils serpentine.

Field Char:

Short tree in forest islands of grassland, $7 \mathrm{~m}$ tall, branching abundantly above and below; bole cylindrical, $12 \mathrm{~cm}$ diam.; bark gray, fissured; sap yellow; flower buds maroon, calyx greenish-red, petals 4 and scarlet during anthesis, flowers $7 \mathrm{~mm}$ diam.; fruit yoke w/ stigma at apex

McDonald \& Ismail 4037 
PLANTS OF INDONESIA

Euphorbiaceae

Cat. \# 4038

Bridelia cf. tomentosa Bl.

Det. By: McDonald 1993

Locality:

Kabaena; Kabaena,

Gunung Sabampolulu, $1 \mathrm{~km} \mathrm{~W}$ of Tangkeno.

$5^{\circ} 16^{\prime} 121^{\circ} 56^{\prime}$ Alt: $700 \mathrm{~m}$

Habitat:

E slopes of range, short forest savannah.

Arborescent associates include Ficus, Metrosideros,

Gardenia, Decasperma, Cratoxyla. Soils serpentine.

Field Char:

Short tree branching from base, $5 \mathrm{~m}$ tall, basal stems to $5 \mathrm{~cm}$ diam., cylindrical; bark smooth, pinkish-tan, inner bark pink; flowers pale yellow-green; fruits

immature, green.

McDonald \& Ismail 4038

30 July 1993

\author{
HERBARIUM BOGORIENSE \\ HARVARD UNIVERSITY HERBARIA
}

PLANTS OF INDONESIA

Verbenaceae

Cat. \# 4039

Vitex cofassus Reinw.

Det. By: McDonald 1993

Locality:

Kabaena; Kabaena,

Gunung Sabampolulu, $1 \mathrm{~km} \mathrm{~W}$ of Tangkeno.

$5^{\circ} 16^{\prime} 121^{\circ} 56^{\prime}$ Alt: $700 \mathrm{~m}$

Habitat:

E slopes of range, short forest savannah.

Arborescent associates include Ficus, Metrosideros,

Gardenia, Decasperma, Cratoxyla. Soils serpentine.

Field Char:

Slender canopy tree $15 \mathrm{~m}$ tall, branching at summit; bole straight, cylindrical, $15 \mathrm{~cm}$ diam.; bark tannishgray, rough; calyx pale green, corolla $7 \mathrm{~mm}$ long, tube purple at base, limb blue, segments 5 , bluepurple, reflexed

McDonald \& Ismail 4039

\title{
HERBARIUM BOGORIENSE HARVARD UNIVERSITY HERBARIA
}




\title{
PLANTS OF INDONESIA
}

Cat. \# 4040

Cratoxylon formosum (Jack) Dyer

Det. By: McDonald 1993

Locality:

Kabaena; Kabaena,

Gunung Sabampolulu, $1 \mathrm{~km} \mathrm{~W}$ of Tangkeno.

$5^{\circ} 16^{\prime} 121^{\circ} 56^{\prime}$ Alt: $700 \mathrm{~m}$

Habitat:

E slopes of range, short forest savannah.

Arborescent associates include Ficus, Metrosideros,

Gardenia, Decasperma, Cratoxyla. Soils serpentine.

Field Char:

Short tree on forest-margin $7 \mathrm{~m}$ tall, branching above and below, lateral branches ascending; bole

cylindrical, $10 \mathrm{~cm}$ diam.; bark gray, small flaky;

foliage green and reddish; fruit abundant, dry,

brown, already dehisced.

McDonald \& Ismail 4040

31 July 1993

\author{
HERBARIUM BOGORIENSE \\ HARVARD UNIVERSITY HERBARIA
}

\section{PLANTS OF INDONESIA}

Rutaceae

Cat. \# 4041

Clausena aff. excavata Burm. $f$.

Det. By: McDonald 1993

Locality:

Kabaena; Kabaena,

Gunung Sabampolulu, $1 \mathrm{~km} \mathrm{~W}$ of Tangkeno.

$5^{\circ} 16^{\prime} 121^{\circ} 56^{\prime}$ Alt: $700 \mathrm{~m}$

Habitat:

E slopes of range, short forest savannah.

Arborescent associates include Ficus, Metrosideros,

Gardenia, Decasperma, Cratoxyla. Soils serpentine.

Field Char:

Small tree on forest-margin, $8 \mathrm{~m}$ tall, branching

above; bole cylindrical, $10 \mathrm{~cm}$ diam.; bark pale gray, smoothish; fruits in terminal corymb, maturing from green to orange.

McDonald \& Ismail 4041

31 July 1993

HERBARIUM BOGORIENSE HARVARD UNIVERSITY HERBARIA 


\section{PLANTS OF INDONESIA}

Olacaceae

Cat. \# 4042

Olax scandens Roxb.

Det. By: McDonald 1993

Locality:

Kabaena; Kabaena,

Gunung Sabampolulu, $1 \mathrm{~km}$ W of Tangkeno.

$5^{\circ} 16^{\prime} 121^{\circ} 56^{\prime}$ Alt: $700 \mathrm{~m}$

Habitat:

E slopes of range, short forest savannah.

Arborescent associates include Ficus, Metrosideros,

Gardenia, Decasperma, Cratoxyla. Soils serpentine.

Field Char:

Shrubby liana to $8 \mathrm{~m}$ tall, branches stout, pendent; calyx yellowish-green, corolla white, segments 3 , bearing 2 bifid appendages inside, anthers yellowish, ovary pale yellow.

McDonald \& Ismail 4042

31 July 1993

\section{HERBARIUM BOGORIENSE \\ HARVARD UNIVERSITY HERBARIA}

\section{PLANTS OF INDONESIA}

Urticaceae

Cat. \# 4043

\section{Leucosyke aff. capitulata}

Det. By: McDonald 1993

Locality:

Kabaena; Kabaena,

Gunung Sabampolulu, $1 \mathrm{~km} \mathrm{~W}$ of Tangkeno.

$5^{\circ} 16^{\prime} 121^{\circ} 56^{\prime}$ Alt: $700 \mathrm{~m}$

Habitat:

E slopes of range, short forest savannah.

Arborescent associates include Ficus, Metrosideros,

Gardenia, Decasperma, Cratoxyla. Soils serpentine.

Field Char:

Shrub on forest edge, $2 \mathrm{~m}$ tall, branching 8 times

from base, stems to $2 \mathrm{~cm}$ diam.; bark pale gray, smoothish; leaves coriaceous; inflorescence pale green, turning whitish with age. 


\section{PLANTS OF INDONESIA}

Orchidaceae

Cat. \# 4044

Vandopsis lissochiloides (Guad.) Pfitz.

Det. By: McDonald 1993

Locality:

Kabaena; Kabaena,

Gunung Sabampolulu, $1 \mathrm{~km}$ W of Tangkeno.

$5^{\circ} 16^{\prime} 121^{\circ} 56^{\prime}$ Alt: $700 \mathrm{~m}$

Habitat:

E slopes of range, short forest savannah.

Arborescent associates include Ficus, Metrosideros,

Gardenia, Decasperma, Cratoxyla. Soils serpentine.

Field Char:

Colonial, monopodial, on rock outcrops, stems to 2

$\mathrm{m}$ long; foliage yellow-green; inflorescence 1-2 m

long; flower bud bright lavender; tepals 5, yellow

speckled maroon, keel bright lavender, column white, speckled purple; fruits yellow green.

McDonald \& Ismail 4044

31 July 1993

\section{HERBARIUM BOGORIENSE \\ HARVARD UNIVERSITY HERBARIA}

\section{PLANTS OF INDONESIA}

Urticaceae

Cat. \# 4045

Pipturus sp.

Det. By: McDonald 1993

Locality:

Kabaena; Kabaena,

Gunung Sabampolulu, $1 \mathrm{~km}$ W of Tangkeno.

$5^{\circ} 16^{\prime} 121^{\circ} 56^{\prime}$ Alt: $700 \mathrm{~m}$

Habitat:

E slopes of range, short forest savannah.

Arborescent associates include Ficus, Metrosideros,

Gardenia, Decasperma, Cratoxyla. Soils serpentine.

Field Char:

Short tree at rock outcrops, $4 \mathrm{~m}$ tall, branching

midway up; bole cylindrical, $10 \mathrm{~cm}$ diam.; bark gray, rough; leaves green above, silver-gray below;

inflorescence greenish cream.

McDonald \& Ismail 4045

31 July 1993

HERBARIUM BOGORIENSE HARVARD UNIVERSITY HERBARIA 


\section{PLANTS OF INDONESIA}

Cat. \# 4046

Dioscoreaceae?

Det. By: McDonald 1993

Locality:

Kabaena; Kabaena,

Gunung Sabampolulu, $1 \mathrm{~km} \mathrm{~W}$ of Tangkeno.

$5^{\circ} 16^{\prime} 121^{\circ} 56^{\prime}$ Alt: $700 \mathrm{~m}$

Habitat:

E slopes of range, short forest savannah.

Arborescent associates include Ficus, Metrosideros,

Gardenia, Decasperma, Cratoxyla. Soils serpentine.

Field Char:

Succulent vine, flowers yellow.

McDonald \& Ismail 4046

31 July 1993

\section{HERBARIUM BOGORIENSE \\ HARVARD UNIVERSITY HERBARIA}

\section{PLANTS OF INDONESIA}

Orchidaceae

Cat. \# 4047

\section{Calanthe sp.}

Det. By: McDonald 1993

Locality:

Kabaena; Kabaena,

Gunung Sabampolulu, $1 \mathrm{~km} \mathrm{~W}$ of Tangkeno.

$5^{\circ} 16^{\prime} 121^{\circ} 56^{\prime}$ Alt: $700 \mathrm{~m}$

Habitat:

E slopes of range, short forest savannah.

Arborescent associates include Ficus, Metrosideros,

Gardenia, Decasperma, Cratoxyla. Soils serpentine.

Field Char:

Terrestrial orchid, leaves absent, rachis light green,

flower white, tube column lavender inside.

McDonald \& Ismail 4047

31 July 1993

HERBARIUM BOGORIENSE HARVARD UNIVERSITY HERBARIA 


\section{PLANTS OF INDONESIA}

Clusiaceae

Cat. \# 4048

Garcinia sp.

Det. By: McDonald 1993

Locality:

Kabaena; Kabaena,

Gunung Sabampolulu, $1 \mathrm{~km}$ W of Tangkeno.

$5^{\circ} 16^{\prime} 121^{\circ} 56^{\prime}$ Alt: $700 \mathrm{~m}$

Habitat:

E slopes of range, short forest savannah.

Arborescent associates include Ficus, Metrosideros,

Gardenia, Decasperma, Cratoxyla. Soils serpentine.

Field Char:

Canopy tree in forest ravine, $12 \mathrm{~m}$ tall, branching above; bole straight, cylindrical, $13 \mathrm{~cm}$ diam,; bark tan, rough; fruits immature, green.

McDonald \& Ismail 4048

31 July 1993

\section{HERBARIUM BOGORIENSE HARVARD UNIVERSITY HERBARIA}

\section{PLANTS OF INDONESIA}

Meliaceae

Cat. \# 4049

Dysoxylum nutans Miq.

Det. By: McDonald 1993

Locality:

Kabaena; Kabaena,

Gunung Sabampolulu, $1 \mathrm{~km}$ W of Tangkeno.

$5^{\circ} 16^{\prime} 121^{\circ} 56^{\prime}$ Alt: $700 \mathrm{~m}$

Habitat:

E slopes of range, short forest savannah.

Arborescent associates include Ficus, Metrosideros,

Gardenia, Decasperma, Cratoxyla. Soils serpentine.

Field Char:

Understory tree, $5 \mathrm{~m}$ tall, branching above; bole

cylindrical, $12 \mathrm{~cm}$ diam.; bark gray, rough; flowers in bud, tubular, pale green, ca. $1 \mathrm{~cm}$ long; fruits occasional, pale orange, sutures 5 , reddish.

McDonald \& Ismail 4049

31 July 1993

HERBARIUM BOGORIENSE HARVARD UNIVERSITY HERBARIA 


\section{PLANTS OF INDONESIA}

Lauraceae

Cat. \# 4050

Actinodaphne cf. macrophylla Bl.

Det. By: McDonald 1993

Locality:

Kabaena; Kabaena,

Gunung Sabampolulu, $1 \mathrm{~km}$ W of Tangkeno.

$5^{\circ} 16^{\prime} 121^{\circ} 56^{\prime}$ Alt: $700 \mathrm{~m}$

Habitat:

E slopes of range, short forest savannah.

Arborescent associates include Ficus, Metrosideros,

Gardenia, Decasperma, Cratoxyla. Soils serpentine.

Field Char:

Midstory tree $12 \mathrm{~m}$ tall, branching above; bole

cylindrical, $20 \mathrm{~cm}$ diam.; bark tan-gray, rough;

flowers buds pale yellow green, petals 6 , brown;

fruits immature, green.

McDonald \& Ismail 4050

31 July 1993

HERBARIUM BOGORIENSE

HARVARD UNIVERSITY HERBARIA

\section{PLANTS OF INDONESIA}

Podocarpaceae

Cat. \# 4051

Podocarpus sp.

Det. By:

Locality:

Kabaena; Kabaena,

Mt. Sangia Wita, ca. $8 \mathrm{~km}$ SE of Tankeno.

$5^{\circ} 19^{\prime} 121^{\circ} 58^{\prime}$ Alt: $1200 \mathrm{~m}$

Habitat:

Forested NW slopes, canopy 10-20 m tall, associates include Eugenia, Psychotira, Podocarpus, Vernonia, Pittosporum, Melastoma. Soils serpentine.

Field Char:

Midstory tree $10 \mathrm{~m}$ tall, branching midway up,

lateral branches perpendicular to trunk, in whorls of 3; bole cylindrical, $7 \mathrm{~cm}$ diam.; bark rusty brown, narrowly, vertically grooved; leaves dark green; seed green, aril fleshy, red at maturity. 


\section{PLANTS OF INDONESIA}

Araliaceae

Cat. \# 4052

Gastonia serratifolia (Miq.) Philipson

Det. By: McDonald 1993

Locality:

Kabaena; Kabaena,

Mt. Sangia Wita, ca. $8 \mathrm{~km} \mathrm{SE}$ of Tankeno.

$5^{\circ} 19^{\prime} 121^{\circ} 58^{\prime}$ Alt: $1200 \mathrm{~m}$

Habitat:

Forested NW slopes, canopy 10-20 m tall, associates include Eugenia, Psychotira, Podocarpus, Vernonia, Pittosporum, Melastoma. Soils serpentine.

Field Char:

Canopy tree $10 \mathrm{~m}$ tall, branching sparingly above; bole cylindrical, $17 \mathrm{~cm}$ diam.; bark gray, deeply fissured, forming a diamond-lattice pattern; leaves coriaceous; flower buds dark maroon, petals maroon, stamens pale yellow, fruits maroonish.

McDonald \& Ismail 4052

1 August 1993

\section{HERBARIUM BOGORIENSE \\ HARVARD UNIVERSITY HERBARIA}

\section{PLANTS OF INDONESIA}

Rubiaceae

Cat. \# 4053

Psychotria sp.

Det. By: McDonald 1993

Locality:

Kabaena; Kabaena,

Mt. Sangia Wita, ca. $8 \mathrm{~km} \mathrm{SE}$ of Tankeno.

$5^{\circ} 19^{\prime} 121^{\circ} 58^{\prime}$ Alt: $1200 \mathrm{~m}$

Habitat:

Forested NW slopes, canopy $10-20 \mathrm{~m}$ tall, associates include Eugenia, Psychotira, Podocarpus, Vernonia, Pittosporum, Melastoma. Soils serpentine.

Field Char:

Co-dominant understory tree, $7 \mathrm{~m}$ tall, branching midway up; bole crooked, cylindrical, $10 \mathrm{~cm}$ diam.; bark gray, cracking horizontally; foliage dark green, coriaceous; fruits orange to red during maturation.

McDonald \& Ismail 4053

1 August 1993

\section{HERBARIUM BOGORIENSE HARVARD UNIVERSITY HERBARIA}




\section{PLANTS OF INDONESIA}

Myrtaceae

Cat. \# 4054

Eugenia sp.

Det. By: McDonald 1993

Locality:

Kabaena; Kabaena,

Mt. Sangia Wita, ca. $8 \mathrm{~km}$ SE of Tankeno.

$5^{\circ} 19^{\prime} 121^{\circ} 58^{\prime}$ Alt: $1200 \mathrm{~m}$

Habitat:

Forested NW slopes, canopy $10-20 \mathrm{~m}$ tall, associates include Eugenia, Psychotira, Podocarpus, Vernonia, Pittosporum, Melastoma. Soils serpentine.

Field Char:

Canopy tree $12 \mathrm{~m}$ tall, branching midway up; bole cylindrical, $10 \mathrm{~cm}$ diam.; bark gray, smooth; floral disk pale yellow; fruits creamy green.

McDonald \& Ismail 4054

1 August 1993

\section{HERBARIUM BOGORIENSE \\ HARVARD UNIVERSITY HERBARIA}

\section{PLANTS OF INDONESIA}

Euphorbiaceae

Cat. \# 4055

Austrobuxus cf. nitudus (Miq.) v.St.

Det. By: McDonald 1993

Locality:

Kabaena; Kabaena,

Mt. Sangia Wita, ca. $8 \mathrm{~km}$ SE of Tankeno.

$5^{\circ} 19^{\prime} 121^{\circ} 58^{\prime}$ Alt: $1200 \mathrm{~m}$

Habitat:

Forested NW slopes, canopy 10-20 m tall, associates include Eugenia, Psychotira, Podocarpus, Vernonia, Pittosporum, Melastoma. Soils serpentine.

Field Char:

Canopy tree $13 \mathrm{~m}$ tall, branching above; bole cylindrical-contoured, $30 \mathrm{~cm}$ diam.; bark brownish gray, flaky; fruits hard, yellowish and red.

McDonald \& Ismail 4055

1 August 1993

HERBARIUM BOGORIENSE HARVARD UNIVERSITY HERBARIA 


\section{PLANTS OF INDONESIA}

Asteraceae

Cat. \# 4056

Vernonia arborea Buch. Ham.

Det. By: McDonald 1993

Locality:

Kabaena; Kabaena,

Mt. Sangia Wita, ca. $8 \mathrm{~km}$ SE of Tankeno.

$5^{\circ} 19^{\prime} 121^{\circ} 58^{\prime}$ Alt: $1200 \mathrm{~m}$

Habitat:

Forested NW slopes, canopy 10-20 m tall, associates include Eugenia, Psychotira, Podocarpus, Vernonia, Pittosporum, Melastoma. Soils serpentine.

Field Char:

Timberline, canopy tree, $10 \mathrm{~m}$ tall; bole crooked, contoured, $30 \mathrm{~cm}$ diam.; bark tan-pale gray, lateral branches very crooked; leaves coriaceous; wood hard.

McDonald \& Ismail 4056

1 August 1993

\section{HERBARIUM BOGORIENSE HARVARD UNIVERSITY HERBARIA}

\section{PLANTS OF INDONESIA}

Daphniphyllaceae

Cat. \# 4057

Daphniphyllum cf. glaucescens $B l$.

Det. By: McDonald 1993

Locality:

Kabaena; Kabaena,

Mt. Sangia Wita, ca. $8 \mathrm{~km}$ SE of Tankeno.

$5^{\circ} 19^{\prime} 121^{\circ} 58^{\prime}$ Alt: $1200 \mathrm{~m}$

Habitat:

Forested NW slopes, canopy 10-20 m tall, associates include Eugenia, Psychotira, Podocarpus, Vernonia, Pittosporum, Melastoma. Soils serpentine.

Field Char:

Midstory tree $12 \mathrm{~m}$ tall, branching midway up; bole cylindrical, $10 \mathrm{~cm}$ diam.; bark tan-pale gray, smoothish, sparingly, shallowly grooved vertically; laminas glaucous below, new leaves bear a pink and lavender pigmentation at base of petiole; flowers staminate, anthers dark lavender. 


\section{PLANTS OF INDONESIA}

Cat. \# 4058

\section{Achronisia laurifolia $\mathbf{B l}$.}

Det. By: McDonald 1993

Locality:

Kabaena; Kabaena,

Mt. Sangia Wita, ca. $8 \mathrm{~km}$ SE of Tankeno.

$5^{\circ} 19^{\prime} 121^{\circ} 58^{\prime}$ Alt: $1200 \mathrm{~m}$

Habitat:

Forested NW slopes, canopy 10-20 m tall, associates include Eugenia, Psychotira, Podocarpus, Vernonia, Pittosporum, Melastoma. Soils serpentine.

Field Char:

Small tree $5 \mathrm{~m}$ tall, branching above and below; bole cylindrical, $6 \mathrm{~cm}$ diam.; bark pale gray, smooth, inner bark tan; inflorescence pale green, flowers cream; fruits immature, pale green.

McDonald \& Ismail 4058

1 August 1993

\section{HERBARIUM BOGORIENSE HARVARD UNIVERSITY HERBARIA}

\section{PLANTS OF INDONESIA}

Sabiaceae

Cat. \# 4059

Meliosma sp. nov. McDonald ined.

Det. By: McDonald 1993

Locality:

Kabaena; Kabaena,

Summit of Sangia Wita, ca. $10 \mathrm{~km}$ SE of Tangkeno . $5^{\circ} 16^{\prime} \mathrm{S} 121^{\circ} 54^{\prime} \mathrm{E}$ Alt: $1500 \mathrm{~m}$

Habitat:

Short sclerophyllus forest, subelfin. Associates include Melastoma, Actinodaphne, Meliosma,

Calamus, Daphniphyllum. Solis serpentine.

\section{Field Char:}

Canopy tree $8 \mathrm{~m}$ tall, branching above; bole cylindrical, $14 \mathrm{~cm}$ diam.; bark gray, roughened by lenticels; leaves coriaceous; flowers in bud, green; fruits immature, green and mottled brown.

McDonald \& Ismail 4059

1 August 1993

HERBARIUM BOGORIENSE HARVARD UNIVERSITY HERBARIA 


\section{PLANTS OF INDONESIA}

Moraceae

Cat. \# 40605

Ficus congesta Roxb.

Det. By: McDonald 1993

Locality:

Kabaena; Kabaena,

Gunung Sabampolulu, $1 \mathrm{~km} \mathrm{S-SE} \mathrm{of} \mathrm{Tangkeno}$

$5^{\circ} 16^{\prime} \mathrm{S} 121^{\circ} 56^{\prime} \mathrm{E}$ Alt: $700-900 \mathrm{~m}$

Habitat:

W slopes of range, mixed ecotone of grasslands and

short forest; arborescent associates include Grevillea, Helicia, Gardenia, Vaccinium, Eugenia; soils serpentine.

Field Char:

Canopy tree $15 \mathrm{~m}$ tall, branching above; bole cylindrical, $15 \mathrm{~cm}$ diam.; bark gray, somewhat smooth, bearing cauliflorous branches; figs reddish, spotted yellow (apple colors).

McDonald \& Ismail 4060.5

1 August 1993

HERBARIUM BOGORIENSE

HARVARD UNIVERSITY HERBARIA

PLANTS OF INDONESIA

Moraceae

Cat. \# 40605

\section{Ficus congesta Roxb.}

Det. By: McDonald 1993

Locality:

Kabaena; Kabaena,

Gunung Sabampolulu, $1 \mathrm{~km}$ S-SE of Tangkeno

$5^{\circ} 16^{\prime} \mathrm{S} 121^{\circ} 56^{\prime} \mathrm{E}$ Alt: $700-900 \mathrm{~m}$

Habitat:

W slopes of range, mixed ecotone of grasslands and short forest; arborescent associates include Grevillea, Helicia, Gardenia, Vaccinium, Eugenia; soils serpentine.

Field Char:

Canopy tree $15 \mathrm{~m}$ tall, branching above; bole cylindrical, $15 \mathrm{~cm}$ diam.; bark gray, somewhat smooth, bearing cauliflorous branches; figs reddish, spotted yellow (apple colors).

McDonald \& Ismail 4060.5

1 August 1993

\section{HERBARIUM BOGORIENSE HARVARD UNIVERSITY HERBARIA}




\title{
PLANTS OF INDONESIA
}

Lauraceae

Cat. \# 4060

Actinodaphne sp.

Det. By: McDonald 1993

Locality:

Kabaena; Kabaena,

Summit of Sangia Wita, ca. $10 \mathrm{~km} \mathrm{SE}$ of Tangkeno .

$5^{\circ} 16^{\prime} \mathrm{S} 121^{\circ} 54^{\prime} \mathrm{E}$ Alt: $1500 \mathrm{~m}$

Habitat:

Short sclerophyllus forest, subelfin. Associates include Melastoma, Actinodaphne, Meliosma, Calamus, Daphniphyllum. Solis serpentine.

Field Char:

Shrub $3 \mathrm{~m}$ tall, branching above and below, basal stem $4 \mathrm{~cm}$ diam.; new leaves silver, soft-canescent, older leaves green, glossy above, glaucous below; Tepals 6, pale green, stamens 3; fruits rotund, black, ca. $1 \mathrm{~cm}$ diam., born on a green cup ca. $8 \mathrm{~mm}$ diam. and $3 \mathrm{~mm}$ tall.

McDonald \& Ismail 4060

1 August 1993

\author{
HERBARIUM BOGORIENSE \\ HARVARD UNIVERSITY HERBARIA
}

\section{PLANTS OF INDONESIA}

Euphorbiaceae

Cat. \# 4061

\section{Glochidion aff. obscurum (Willd) $B l$}

Det. By: McDonald 1993

Locality:

Kabaena; Kabaena,

Summit of Sangia Wita mountain, ca $10 \mathrm{~km} \mathrm{SE} \mathrm{of}$

Tangkeno.

$5^{\circ} 19^{\prime} \mathrm{S} 121^{\circ} 58^{\prime} \mathrm{E}$ Alt: $1500 \mathrm{~m}$

Habitat:

Short sclerophyllous forest, subelfin, associates

include melastoma, Actinodaphne, Meliosma,

Calamus, Daphniphyllum, etc. Soils serpentine.

Field Char:

Occasional woody shrub $1.5 \mathrm{~m}$ tall, ellipsoid, branching profusely above and below, most leaves fallen. Fruits reddish maroon, depressed-globose.

McDonald \& Ismail 4061

2 August 1993

HERBARIUM BOGORIENSE HARVARD UNIVERSITY HERBARIA 


\section{PLANTS OF INDONESIA}

Moraceae

Cat. \# 40605

Ficus congesta Roxb.

Det. By: McDonald 1993

Locality:

Kabaena; Kabaena,

Gunung Sabampolulu, $1 \mathrm{~km}$ S-SE of Tangkeno

$5^{\circ} 16^{\prime} \mathrm{S} 121^{\circ} 56^{\prime} \mathrm{E}$ Alt: $700-900 \mathrm{~m}$

Habitat:

W slopes of range, mixed ecotone of grasslands and short forest; arborescent associates include Grevillea, Helicia, Gardenia, Vaccinium, Eugenia; soils serpentine.

Field Char:

Canopy tree $15 \mathrm{~m}$ tall, branching above; bole cylindrical, $15 \mathrm{~cm}$ diam.; bark gray, somewhat smooth, bearing cauliflorous branches; figs reddish, spotted yellow (apple colors).

McDonald \& Ismail 4060.5

1 August 1993

HERBARIUM BOGORIENSE HARVARD UNIVERSITY HERBARIA

\section{PLANTS OF INDONESIA}

Moraceae

Cat. \# 40605

Ficus congesta Roxb.

Det. By: McDonald 1993

Locality:

Kabaena; Kabaena,

Gunung Sabampolulu, $1 \mathrm{~km}$ S-SE of Tangkeno

$5^{\circ} 16^{\prime} \mathrm{S} 121^{\circ} 56^{\prime} \mathrm{E}$ Alt: $700-900 \mathrm{~m}$

Habitat:

W slopes of range, mixed ecotone of grasslands and short forest; arborescent associates include Grevillea, Helicia, Gardenia, Vaccinium, Eugenia; soils serpentine.

Field Char:

Canopy tree $15 \mathrm{~m}$ tall, branching above; bole cylindrical, $15 \mathrm{~cm}$ diam.; bark gray, somewhat smooth, bearing cauliflorous branches; figs reddish, spotted yellow (apple colors).

McDonald \& Ismail 4060.5

1 August 1993

HERBARIUM BOGORIENSE HARVARD UNIVERSITY HERBARIA 


\section{PLANTS OF INDONESIA}

Melastomataceae

Cat. \# 4062

Melastoma sp.

Det. By: McDonald 1993

Locality:

Kabaena; Kabaena,

Summit of Sangia Wita mountain, ca $10 \mathrm{~km} \mathrm{SE} \mathrm{of}$

Tangkeno.

$5^{\circ} 19^{\prime} \mathrm{S} 121^{\circ} 58^{\prime} \mathrm{E}$ Alt: $1500 \mathrm{~m}$

Habitat:

Short sclerophyllous forest, subelfin, associates

include melastoma, Actinodaphne, Meliosma,

Calamus, Daphniphyllum, etc. Soils serpentine.

Field Char:

Common woody shrub 1-2 m tall, often flat on top;

leaves red below and along major veins, green

otherwise. Corolla dark lavender, stamens yellow, calyx reddish.

McDonald \& Ismail 4062

2 August 1993

HERBARIUM BOGORIENSE

HARVARD UNIVERSITY HERBARIA

\section{PLANTS OF INDONESIA}

Podocarpaceae

Cat. \# 4063

Dacrydium sp.

Det. By: McDonald 1993

Locality:

Kabaena; Kabaena,

Summit of Sangia Wita mountain, ca $10 \mathrm{~km} \mathrm{SE} \mathrm{of}$

Tangkeno.

$5^{\circ} 19^{\prime} \mathrm{S} 121^{\circ} 58^{\prime} \mathrm{E}$ Alt: $1500 \mathrm{~m}$

Habitat:

Short sclerophyllous forest, subelfin, associates include melastoma, Actinodaphne, Meliosma,

Calamus, Daphniphyllum, etc. Soils serpentine.

Field Char:

Sterile short tree to $3 \mathrm{~m}$. Branching above and below.

McDonald \& Ismail 4063

2 August 1993

HERBARIUM BOGORIENSE

HARVARD UNIVERSITY HERBARIA 


\section{PLANTS OF INDONESIA}

Cat. \# 4064

Evodia cf. eunera Miq.

Det. By: McDonald 1993

Locality:

Kabaena; Kabaena,

Summit of Sangia Wita mountain, ca $10 \mathrm{~km} \mathrm{SE}$ of

Tangkeno.

$5^{\circ} 19^{\prime} \mathrm{S} 121^{\circ} 58^{\prime} \mathrm{E}$ Alt: $1500 \mathrm{~m}$

Habitat:

Short sclerophyllous forest, subelfin, associates include melastoma, Actinodaphne, Meliosma, Calamus, Daphniphyllum, etc. Soils serpentine.

Field Char:

Uncommon woody shrub $2 \mathrm{~m}$ tall, branching sparingly, flat on top; leaves at stem apex only;

inflorescence branching, green, flowers cream.

McDonald \& Ismail 4064

2 August 1993

HERBARIUM BOGORIENSE

HARVARD UNIVERSITY HERBARIA

\section{PLANTS OF INDONESIA}

Oxalidaceae

Cat. \# 4065

Biophytum fruticosum Bl.

Det. By: McDonald 1993

Locality:

Kabaena; Kabaena,

Summit of Sangia Wita mountain, ca $10 \mathrm{~km} \mathrm{SE}$ of

Tangkeno.

$5^{\circ} 19^{\prime} \mathrm{S} 121^{\circ} 58^{\prime} \mathrm{E}$ Alt: $1500 \mathrm{~m}$

Habitat:

Short sclerophyllous forest, subelfin, associates include melastoma, Actinodaphne, Meliosma,

Calamus, Daphniphyllum, etc. Soils serpentine.

Field Char:

Occasional terrestrial herb, $10-30 \mathrm{~cm}$ tall, leaves only at apex, peduncles maroonish, corollas pinkish due to pigmented petal venation.

McDonald \& Ismail 4065

2 August 1993

HERBARIUM BOGORIENSE HARVARD UNIVERSITY HERBARIA 


\section{PLANTS OF INDONESIA}

Thymelaeaceae

Cat. \# 4066

Wikstroemia sp. nov.?

Det. By: McDonald 1993

Locality:

Kabaena; Kabaena,

Summit of Sangia Wita mountain, ca $10 \mathrm{~km} \mathrm{SE} \mathrm{of}$

Tangkeno.

$5^{\circ} 19^{\prime} \mathrm{S} 121^{\circ} 58^{\prime} \mathrm{E}$ Alt: $1500 \mathrm{~m}$

Habitat:

Short sclerophyllous forest, subelfin, associates

include melastoma, Actinodaphne, Meliosma,

Calamus, Daphniphyllum, etc. Soils serpentine.

Field Char:

Occasional woody shrub $1 \mathrm{~m}$ tall, branching profusely above and below, ellipsoid; leaves green, coriaceous, corolla bright greenish yellow; fruits rare, ellipsoid, ca. $8 \mathrm{~mm}$ long.

McDonald \& Ismail 4066

2 August 1993

\section{HERBARIUM BOGORIENSE \\ HARVARD UNIVERSITY HERBARIA}

\section{PLANTS OF INDONESIA}

Asclepiadaceae

Cat. \# 4067

Dischidia sp.

Det. By: McDonald 1993

Locality:

Kabaena; Kabaena,

Summit of Sangia Wita mountain, ca $10 \mathrm{~km} \mathrm{SE} \mathrm{of}$

Tangkeno.

$5^{\circ} 19^{\prime} \mathrm{S} 121^{\circ} 58^{\prime} \mathrm{E}$ Alt: $1500 \mathrm{~m}$

Habitat:

Short sclerophyllous forest, subelfin, associates

include melastoma, Actinodaphne, Meliosma,

Calamus, Daphniphyllum, etc. Soils serpentine.

Field Char:

Rare epiphytic succulent, pendent, branching much,

flowers pyriform-urceolate, orange-red.

McDonald \& Ismail 4067

2 August 1993

HERBARIUM BOGORIENSE HARVARD UNIVERSITY HERBARIA 


\section{PLANTS OF INDONESIA}

Cat. \# 4068

Ruellia?

Det. By: McDonald 1993

Locality:

Kabaena; Kabaena,

Summit of Sangia Wita mountain, ca $10 \mathrm{~km}$ SE of

Tangkeno.

$5^{\circ} 19^{\prime} \mathrm{S} 121^{\circ} 58^{\prime} \mathrm{E}$ Alt: $1500 \mathrm{~m}$

Habitat:

Short sclerophyllous forest, subelfin, associates

include melastoma, Actinodaphne, Meliosma,

Calamus, Daphniphyllum, etc. Soils serpentine.

Field Char:

Common short woody shrub on rock outcrops, ca. $50 \mathrm{~cm}$ tall, branching above and below; leaves dark green above, pale green below; calyx green, corola white with 2 prominent series of purple dots on lower throat surface.

McDonald \& Ismail 4068

2 August 1993

\section{HERBARIUM BOGORIENSE HARVARD UNIVERSITY HERBARIA}

\section{PLANTS OF INDONESIA}

Oleaceae

Cat. \# 4069

Jasminum sp.

Det. By: McDonald 1993

Locality:

Kabaena; Kabaena,

Summit of Sangia Wita mountain, ca $10 \mathrm{~km}$ SE of

Tangkeno.

$5^{\circ} 19^{\prime}$ S $121^{\circ} 58^{\prime}$ E Alt: $1500 \mathrm{~m}$

Habitat:

Short sclerophyllous forest, subelfin, associates

include melastoma, Actinodaphne, Meliosma,

Calamus, Daphniphyllum, etc. Soils serpentine.

Field Char:

Common shrubby liana, stems to $1.5 \mathrm{~m}$ long, leaves coriaceous; calyx light green, flower buds very pale green, corolla turning white during anthesis.

McDonald \& Ismail 4069

2 August 1993

\section{HERBARIUM BOGORIENSE HARVARD UNIVERSITY HERBARIA}




\section{PLANTS OF INDONESIA}

Orchidaceae

Cat. \# 4070

Calanthe vestita Lindl.

Det. By: McDonald 1993

Locality:

Kabaena; Kabaena,

Batu Sangia Peak, ca. $7 \mathrm{~km} \mathrm{~W}$ of Tangkeno

$5^{\circ} 16^{\prime} \mathrm{S} 121^{\circ} 54^{\prime} \mathrm{E}$ Alt: $1200-1250 \mathrm{~m}$

Habitat:

Rocky knob of Batu Sangia, vegetation herbaceous

or of stunted trees; associates include Ficus,

Dracaena, Shefflera, Kalanchoe, Tradescantia, many orchids.

Field Char:

Terrestrial, lamina absent, bulbil deep in rock crevices, peduncle light green, bracts light green, corolla white, tube purple.

McDonald \& Ismail 4070

3 August 1993

\section{HERBARIUM BOGORIENSE HARVARD UNIVERSITY HERBARIA}

\section{PLANTS OF INDONESIA}

Orchidaceae

Cat. \# 4071

\section{Dendrobium crumeatum $S w$.}

Det. By: McDonald 1993

Locality:

Kabaena; Kabaena,

Batu Sangia Peak, ca. $7 \mathrm{~km} \mathrm{~W}$ of Tangkeno

$5^{\circ} 16^{\prime} \mathrm{S} 121^{\circ} 54^{\prime} \mathrm{E}$ Alt: $1200-1250 \mathrm{~m}$

Habitat:

Rocky knob of Batu Sangia, vegetation herbaceous

or of stunted trees; associates include Ficus,

Dracaena, Shefflera, Kalanchoe, Tradescantia, many orchids.

Field Char:

Rare epiphyte, sterile, bulbil green turning dull purplish, hollow when old and myrmecophilous; lamina coriaceous, green, 3-4 cm long.

HERBARIUM BOGORIENSE HARVARD UNIVERSITY HERBARIA 


\section{PLANTS OF INDONESIA}

Orchidaceae

Cat. \# 4072

Bulbophyllum sp.

Det. By: McDonald 1993

Locality:

Kabaena; Kabaena,

Batu Sangia Peak, ca. $7 \mathrm{~km} \mathrm{~W}$ of Tangkeno

$5^{\circ} 16^{\prime} \mathrm{S} 121^{\circ} 54^{\prime} \mathrm{E}$ Alt: $1200-1250 \mathrm{~m}$

Habitat:

Rocky knob of Batu Sangia, vegetation herbaceous

or of stunted trees; associates include Ficus,

Dracaena, Shefflera, Kalanchoe, Tradescantia, many orchids.

Field Char:

Common epiphyte growing with mosses, sterile,

bulbils and laminas light green.

McDonald \& Ismail 4072

3 August 1993

\section{HERBARIUM BOGORIENSE \\ HARVARD UNIVERSITY HERBARIA}

\section{PLANTS OF INDONESIA}

Orchidaceae

Cat. \# 4073

Liparis sp.

Det. By: McDonald 1993

Locality:

Kabaena; Kabaena,

Batu Sangia Peak, ca. $7 \mathrm{~km} \mathrm{~W}$ of Tangkeno

$5^{\circ} 16^{\prime} \mathrm{S} 121^{\circ} 54^{\prime} \mathrm{E}$ Alt: $1200-1250 \mathrm{~m}$

Habitat:

Rocky knob of Batu Sangia, vegetation herbaceous

or of stunted trees; associates include Ficus,

Dracaena, Shefflera, Kalanchoe, Tradescantia, many orchids.

Field Char:

Uncommon epiphyte, sterile; bulbils and laminas light green.

McDonald \& Ismail 4073

3 August 1993

HERBARIUM BOGORIENSE HARVARD UNIVERSITY HERBARIA 


\section{PLANTS OF INDONESIA}

Orchidaceae

Cat. \# 4074

Orchidaceae

Det. By: McDonald 1993

Locality:

Kabaena; Kabaena,

Batu Sangia Peak, ca. $7 \mathrm{~km} \mathrm{~W}$ of Tangkeno

$5^{\circ} 16^{\prime} \mathrm{S} 121^{\circ} 54^{\prime} \mathrm{E}$ Alt: $1200-1250 \mathrm{~m}$

Habitat:

Rocky knob of Batu Sangia, vegetation herbaceous

or of stunted trees; associates include Ficus,

Dracaena, Shefflera, Kalanchoe, Tradescantia, many orchids.

Field Char:

Occasional epiphyte, sterile, pendent and grass-like on large trees; foliage green.

McDonald \& Ismail 4074

3 August 1993

\section{HERBARIUM BOGORIENSE \\ HARVARD UNIVERSITY HERBARIA}

\section{PLANTS OF INDONESIA}

Orchidaceae

Cat. \# 4075

Cymbidium finlaysonianum Lindl.

Det. By: McDonald 1993

Locality:

Kabaena; Kabaena,

Batu Sangia Peak, ca. $7 \mathrm{~km}$ W of Tangkeno

$5^{\circ} 16^{\prime} \mathrm{S} 121^{\circ} 54^{\prime} \mathrm{E}$ Alt: $1200-1250 \mathrm{~m}$

Habitat:

Rocky knob of Batu Sangia, vegetation herbaceous

or of stunted trees; associates include Ficus,

Dracaena, Shefflera, Kalanchoe, Tradescantia, many orchids.

Field Char:

Large epiphyte, sterile, colonial, foliage light green.

McDonald \& Ismail 4075

3 August 1993

HERBARIUM BOGORIENSE

HARVARD UNIVERSITY HERBARIA 


\section{PLANTS OF INDONESIA}

Orchidaceae

Cat. \# 4076

Eria sp.

Det. By: McDonald 1993

Locality:

Kabaena; Kabaena,

Batu Sangia Peak, ca. $7 \mathrm{~km} \mathrm{~W}$ of Tangkeno

$5^{\circ} 16$ 'S $121^{\circ} 54^{\prime} \mathrm{E}$ Alt: $1200-1250 \mathrm{~m}$

Habitat:

Rocky knob of Batu Sangia, vegetation herbaceous or of stunted trees; associates include Ficus,

Dracaena, Shefflera, Kalanchoe, Tradescantia, many orchids.

Field Char:

Colonial, terrestrial orchid, colony to $5 \mathrm{~m}$ in diam, basal stems embedded in rock crevices; foliage light green, orangish-pubescent.

McDonald \& Ismail 4076

3 August 1993

\section{HERBARIUM BOGORIENSE HARVARD UNIVERSITY HERBARIA}

\section{PLANTS OF INDONESIA}

Orchidaceae

Cat. \# 4077

Dendrobium bromheadia

Det. By: McDonald 1993

Locality:

Kabaena; Kabaena,

Batu Sangia Peak, ca. $7 \mathrm{~km} \mathrm{~W}$ of Tangkeno

$5^{\circ} 16^{\prime} \mathrm{S} 121^{\circ} 54^{\prime} \mathrm{E}$ Alt: $1200-1250 \mathrm{~m}$

Habitat:

Rocky knob of Batu Sangia, vegetation herbaceous

or of stunted trees; associates include Ficus,

Dracaena, Shefflera, Kalanchoe, Tradescantia, many orchids.

Field Char:

Rare epiphyte, sterile, laminas lax, green.

McDonald \& Ismail 4077

3 August 1993

HERBARIUM BOGORIENSE HARVARD UNIVERSITY HERBARIA 


\section{PLANTS OF INDONESIA}

Cat. \# 4078

Dendrobium sp.

Det. By: McDonald 1993

Locality:

Kabaena; Kabaena,

Batu Sangia Peak, ca. $7 \mathrm{~km} \mathrm{~W}$ of Tangkeno

$5^{\circ} 16^{\prime} \mathrm{S} 121^{\circ} 54^{\prime} \mathrm{E}$ Alt: $1200-1250 \mathrm{~m}$

Habitat:

Rocky knob of Batu Sangia, vegetation herbaceous or of stunted trees; associates include Ficus,

Dracaena, Shefflera, Kalanchoe, Tradescantia, many orchids.

Field Char:

Colonial epiphyte, sterile, stems light green, leaves green.

McDonald \& Ismail 4078

3 August 1993

\section{HERBARIUM BOGORIENSE \\ HARVARD UNIVERSITY HERBARIA}

\section{PLANTS OF INDONESIA}

Orchidaceae

Cat. \# 4079

Dendrobium salacense (Bl.) Lindl.

Det. By: McDonald 1993

Locality:

Kabaena; Kabaena,

Batu Sangia Peak, ca. $7 \mathrm{~km}$ W of Tangkeno

$5^{\circ} 16^{\prime} \mathrm{S} 121^{\circ} 54^{\prime} \mathrm{E}$ Alt: $1200-1250 \mathrm{~m}$

Habitat:

Rocky knob of Batu Sangia, vegetation herbaceous

or of stunted trees; associates include Ficus,

Dracaena, Shefflera, Kalanchoe, Tradescantia, many orchids.

Field Char:

Occasional epiphyte, sterile, bulbil glossy and light green, suspended by stems.

McDonald \& Ismail 4079

3 August 1993

HERBARIUM BOGORIENSE HARVARD UNIVERSITY HERBARIA 


\section{PLANTS OF INDONESIA}

Orchidaceae

Cat. \# 4080

Orchidaceae

Det. By: McDonald 1993

Locality:

Kabaena; Kabaena,

Batu Sangia Peak, ca. $7 \mathrm{~km}$ W of Tangkeno

$5^{\circ} 16^{\prime}$ S $121^{\circ} 54^{\prime}$ E Alt: $1200-1250 \mathrm{~m}$

Habitat:

Rocky knob of Batu Sangia, vegetation herbaceous

or of stunted trees; associates include Ficus,

Dracaena, Shefflera, Kalanchoe, Tradescantia, many orchids.

Field Char:

Colonial epiphyte, sterile, laminas succulent, fruits pale green.

McDonald \& Ismail 4080

3 August 1993

HERBARIUM BOGORIENSE HARVARD UNIVERSITY HERBARIA

\section{PLANTS OF INDONESIA}

Orchidaceae

Cat. \# 4081

Eria sp.

Det. By: McDonald 1993

Locality:

Kabaena; Kabaena,

Batu Sangia Peak, ca. $7 \mathrm{~km}$ W of Tangkeno

$5^{\circ} 16^{\prime} \mathrm{S} 121^{\circ} 54^{\prime} \mathrm{E}$ Alt: $1200-1250 \mathrm{~m}$

Habitat:

Rocky knob of Batu Sangia, vegetation herbaceous or of stunted trees; associates include Ficus,

Dracaena, Shefflera, Kalanchoe, Tradescantia, many orchids.

Field Char:

Occasional epiphyte, sterile, bulbils elongated,

lamina green, coriaceous.

McDonald \& Ismail 4081

3 August 1993

HERBARIUM BOGORIENSE HARVARD UNIVERSITY HERBARIA 


\section{PLANTS OF INDONESIA}

Orchidaceae

Cat. \# 4082

Calanthe sp.

Det. By: McDonald 1993

Locality:

Kabaena; Kabaena,

Batu Sangia Peak, ca. $7 \mathrm{~km} \mathrm{~W}$ of Tangkeno

$5^{\circ} 16$ 'S $121^{\circ} 54$ 'E Alt: $1200-1250 \mathrm{~m}$

Habitat:

Rocky knob of Batu Sangia, vegetation herbaceous

or of stunted trees; associates include Ficus,

Dracaena, Shefflera, Kalanchoe, Tradescantia, many orchids.

Field Char:

Terrestrial, just below tree line, colonial, sympodial in growth, laminas dark green, herbaceous, fruits blackened.

McDonald \& Ismail 4082

3 August 1993

HERBARIUM BOGORIENSE

HARVARD UNIVERSITY HERBARIA

\section{PLANTS OF INDONESIA}

Orchidaceae

Cat. \# 4083

Pholidota imbricata (Roxb.) Lindl.

Det. By: McDonald 1993

Locality:

Kabaena; Kabaena,

Batu Sangia Peak, ca. $7 \mathrm{~km} \mathrm{~W}$ of Tangkeno

$5^{\circ} 16^{\prime} \mathrm{S} 121^{\circ} 54^{\prime} \mathrm{E}$ Alt: $1200-1250 \mathrm{~m}$

Habitat:

Rocky knob of Batu Sangia, vegetation herbaceous

or of stunted trees; associates include Ficus,

Dracaena, Shefflera, Kalanchoe, Tradescantia, many orchids.

Field Char:

Common epiphyte, bulbil somewhat dull, light green, ; lamina dark green above.

McDonald \& Ismail 4083

3 August 1993

HERBARIUM BOGORIENSE

HARVARD UNIVERSITY HERBARIA 


\section{PLANTS OF INDONESIA}

Orchidaceae

Cat. \# 4084

Coelogyne speciosa Lindl.

Det. By: McDonald 1993

Locality:

Kabaena; Kabaena,

Batu Sangia Peak, ca. $7 \mathrm{~km} \mathrm{~W}$ of Tangkeno

$5^{\circ} 16^{\prime} \mathrm{S} 121^{\circ} 54^{\prime} \mathrm{E}$ Alt: $1200-1250 \mathrm{~m}$

Habitat:

Rocky knob of Batu Sangia, vegetation herbaceous

or of stunted trees; associates include Ficus,

Dracaena, Shefflera, Kalanchoe, Tradescantia, many orchids.

Field Char:

Common epiphyte, colonial, bulbil light green,

angled, lamina herbaceous, light green, fruit brown.

McDonald \& Ismail 4084

3 August 1993

\section{HERBARIUM BOGORIENSE \\ HARVARD UNIVERSITY HERBARIA}

\section{PLANTS OF INDONESIA}

Cat. \# 4085

\section{Flinckingeria comata (Bl.) Hawkes}

Det. By: McDonald 1993

Locality:

Kabaena; Kabaena,

Batu Sangia Peak, ca. $7 \mathrm{~km} \mathrm{~W}$ of Tangkeno

$5^{\circ} 16^{\prime} \mathrm{S} 121^{\circ} 54^{\prime} \mathrm{E}$ Alt: $1200-1250 \mathrm{~m}$

Habitat:

Rocky knob of Batu Sangia, vegetation herbaceous or of stunted trees; associates include Ficus,

Dracaena, Shefflera, Kalanchoe, Tradescantia, many orchids.

Field Char:

Colonial, terrestrial, sterile, growing in decaying log, stem purplish-green, lamina green.

McDonald \& Ismail 4085

3 August 1993

HERBARIUM BOGORIENSE HARVARD UNIVERSITY HERBARIA 


\section{PLANTS OF INDONESIA}

Orchidaceae

Cat. \# 4086

Eria multiflorum Lindl.

Det. By: McDonald 1993

Locality:

Kabaena; Kabaena,

Batu Sangia Peak, ca. $7 \mathrm{~km} \mathrm{~W}$ of Tangkeno

$5^{\circ} 16^{\prime} \mathrm{S} 121^{\circ} 54$ 'E Alt: $1200-1250 \mathrm{~m}$

Habitat:

Rocky knob of Batu Sangia, vegetation herbaceous

or of stunted trees; associates include Ficus,

Dracaena, Shefflera, Kalanchoe, Tradescantia, many orchids.

Field Char:

Colonial, terrestrial, stems and leaves somewhat succulent, stems yellowish green, lamina green;

fruits pale green with maroon appendages at apex.

McDonald \& Ismail 4086

3 August 1993

HERBARIUM BOGORIENSE

HARVARD UNIVERSITY HERBARIA

\section{PLANTS OF INDONESIA}

Orchidaceae

Cat. \# 4087

Paphiopedilum lowii (Lindl.) Pfiz.

Det. By: McDonald 1993

Locality:

Kabaena; Kabaena,

Batu Sangia Peak, ca. $7 \mathrm{~km} \mathrm{~W}$ of Tangkeno

$5^{\circ} 16^{\prime}$ 'S $121^{\circ} 54^{\prime}$ E Alt: $1200-1250 \mathrm{~m}$

Habitat:

Rocky knob of Batu Sangia, vegetation herbaceous

or of stunted trees; associates include Ficus,

Dracaena, Shefflera, Kalanchoe, Tradescantia, many orchids.

Field Char:

Terrestrial or epiphytica, colonial, leaves succulentcoriaceous, light green; roots rusty and fuzzy.

McDonald \& Ismail 4087

3 August 1993

HERBARIUM BOGORIENSE

HARVARD UNIVERSITY HERBARIA 


\section{PLANTS OF INDONESIA}

Orchidaceae

Cat. \# 4088

Pholidota imbricata (Roxb.) Lindl.

Det. By: McDonald 1993

Locality:

Kabaena; Kabaena,

Batu Sangia Peak, ca. $7 \mathrm{~km} \mathrm{~W}$ of Tangkeno

$5^{\circ} 16^{\prime} \mathrm{S} 121^{\circ} 54^{\prime} \mathrm{E}$ Alt: $1200-1250 \mathrm{~m}$

Habitat:

Rocky knob of Batu Sangia, vegetation herbaceous or of stunted trees; associates include Ficus,

Dracaena, Shefflera, Kalanchoe, Tradescantia, many orchids.

Field Char:

Common colonial epiphyte, bulbil angular, dull light green, lamina green; inflorescence bracteate.

McDonald \& Ismail 4088

3 August 1993

\section{HERBARIUM BOGORIENSE \\ HARVARD UNIVERSITY HERBARIA}

\section{PLANTS OF INDONESIA}

Orchidaceae

Cat. \# 4089

Liparis sp.

Det. By: McDonald 1993

Locality:

Kabaena; Kabaena,

Batu Sangia Peak, ca. $7 \mathrm{~km} \mathrm{~W}$ of Tangkeno

$5^{\circ} 16$ 'S $121^{\circ} 54$ 'E Alt: $1200-1250 \mathrm{~m}$

Habitat:

Rocky knob of Batu Sangia, vegetation herbaceous

or of stunted trees; associates include Ficus,

Dracaena, Shefflera, Kalanchoe, Tradescantia, many orchids.

Field Char:

Occasional epiphyte, basal bracts whitish, bulbil and lamina light green, coriaceous, fruits light green.

Roots whitish-fuzzy.

McDonald \& Ismail 4089

3 August 1993

HERBARIUM BOGORIENSE

HARVARD UNIVERSITY HERBARIA 


\section{PLANTS OF INDONESIA}

Orchidaceae

Cat. \# 4090

Orchidaceae

Det. By: McDonald 1993

Locality:

Kabaena; Kabaena,

Batu Sangia Peak, ca. $7 \mathrm{~km} \mathrm{~W}$ of Tangkeno

$5^{\circ} 16$ 'S $121^{\circ} 54^{\prime}$ E Alt: $1200-1250 \mathrm{~m}$

Habitat:

Rocky knob of Batu Sangia, vegetation herbaceous

or of stunted trees; associates include Ficus,

Dracaena, Shefflera, Kalanchoe, Tradescantia, many orchids.

Field Char:

Common, terrestrial, colonial; leaf sheath light green turning orangish brown, lamina purplish at stem apex, green below.

McDonald \& Ismail 4090

3 August 1993

\section{HERBARIUM BOGORIENSE HARVARD UNIVERSITY HERBARIA}

\section{PLANTS OF INDONESIA}

Orchidaceae

Cat. \# 4091

Trichoglottis sp.

Det. By: McDonald 1993

Locality:

Kabaena; Kabaena,

Batu Sangia Peak, ca. $7 \mathrm{~km} \mathrm{~W}$ of Tangkeno

$5^{\circ} 16^{\prime} \mathrm{S} 121^{\circ} 54^{\prime} \mathrm{E}$ Alt: $1200-1250 \mathrm{~m}$

Habitat:

Rocky knob of Batu Sangia, vegetation herbaceous

or of stunted trees; associates include Ficus,

Dracaena, Shefflera, Kalanchoe, Tradescantia, many orchids.

Field Char:

Dangling epiphyte, sterile, branching, stems

coriaceous, purple above, green below, leaves

coriaceous-succulent, green-purplish above, green

below.

McDonald \& Ismail 4091

3 August 1993

HERBARIUM BOGORIENSE HARVARD UNIVERSITY HERBARIA 


\section{PLANTS OF INDONESIA}

Crassulaceae

Cat. \# 4092

Kalanchoe integra (Medik.) O.K.Rev

Det. By: McDonald 1993

Locality:

Kabaena; Kabaena,

Batu Sangia Peak, ca. $7 \mathrm{~km}$ W of Tangkeno

$5^{\circ} 16^{\prime} \mathrm{S} 121^{\circ} 54^{\prime} \mathrm{E}$ Alt: $1200-1250 \mathrm{~m}$

Habitat:

Rocky knob of Batu Sangia, vegetation herbaceous or of stunted trees; associates include Ficus,

Dracaena, Shefflera, Kalanchoe, Tradescantia, many orchids.

Field Char:

Common succulent on exposed rock, $10-30 \mathrm{~cm}$ tall, forming small rosettes at base; stems pale green or purple, leaves pale green or purple, succulent, calyx light green, corollas bright yellow.

McDonald \& Ismail 4092

3 August 1993

HERBARIUM BOGORIENSE

HARVARD UNIVERSITY HERBARIA

\section{PLANTS OF INDONESIA}

Asteraceae

Cat. \# 4093

Mikania cordata (Bl.) B.L. Rob.

Det. By: McDonald 1993

Locality:

Kabaena; Kabaena,

Batu Sangia Peak, ca. $7 \mathrm{~km}$ W of Tangkeno

$5^{\circ} 16^{\prime} \mathrm{S} 121^{\circ} 54^{\prime} \mathrm{E}$ Alt: $1200-1250 \mathrm{~m}$

Habitat:

Rocky knob of Batu Sangia, vegetation herbaceous

or of stunted trees; associates include Ficus,

Dracaena, Shefflera, Kalanchoe, Tradescantia, many orchids.

Field Char:

Occasional shrubby vine in rocks or as epiphyte;

leaves light green, phyllaries pale green, pappus and flowers whitish.

McDonald \& Ismail 4093

3 August 1993

HERBARIUM BOGORIENSE HARVARD UNIVERSITY HERBARIA 


\section{PLANTS OF INDONESIA}

Asteraceae

Cat. \# 4094

Wollastonia biflora Dalz. \& Gibs.

Det. By: McDonald 1993

Locality:

Kabaena; Kabaena,

Batu Sangia Peak, ca. $7 \mathrm{~km}$ W of Tangkeno

$5^{\circ} 16^{\prime} \mathrm{S} 121^{\circ} 54^{\prime} \mathrm{E}$ Alt: $1200-1250 \mathrm{~m}$

Habitat:

Rocky knob of Batu Sangia, vegetation herbaceous or of stunted trees; associates include Ficus,

Dracaena, Shefflera, Kalanchoe, Tradescantia, many orchids.

Field Char:

Occasional, short lignescent shrub, to $1 \mathrm{~m}$ tall, branching; leaves green above, pale green below; phyllaries pale green at base, green at apex, corolla yellow.

McDonald \& lsmail 4094

3 August 1993

HERBARIUM BOGORIENSE

HARVARD UNIVERSITY HERBARIA

\section{PLANTS OF INDONESIA}

Commelinaceae

Cat. \# 4095

\section{Commelina sp.}

Det. By: McDonald 1993

Locality:

Kabaena; Kabaena,

Batu Sangia Peak, ca. $7 \mathrm{~km}$ W of Tangkeno

$5^{\circ} 16^{\prime}$ S $121^{\circ} 54^{\prime}$ E Alt: $1200-1250 \mathrm{~m}$

Habitat:

Rocky knob of Batu Sangia, vegetation herbaceous

or of stunted trees; associates include Ficus,

Dracaena, Shefflera, Kalanchoe, Tradescantia, many orchids.

Field Char:

Occasional creeping herb on rocks, stems purple, leaves purplish green above, green below.

McDonald \& Ismail 4095

3 August 1993

HERBARIUM BOGORIENSE HARVARD UNIVERSITY HERBARIA 


\section{PLANTS OF INDONESIA}

Asteraceae

Cat. \# 4096

Asteraceae

Det. By: McDonald 1993

Locality:

Kabaena; Kabaena,

Batu Sangia Peak, ca. $7 \mathrm{~km}$ W of Tangkeno

$5^{\circ} 16^{\prime} \mathrm{S} 121^{\circ} 54^{\prime} \mathrm{E}$ Alt: $1200-1250 \mathrm{~m}$

Habitat:

Rocky knob of Batu Sangia, vegetation herbaceous

or of stunted trees; associates include Ficus,

Dracaena, Shefflera, Kalanchoe, Tradescantia, many orchids.

Field Char:

Short succulent shrub, $1 \mathrm{~m}$ tall, branching sparingly; leaves succulent-coriaceous, silver pubescent below, green above; in bud, capitula light green below, apex of phyllaries purple.

McDonald \& Ismail 4096

3 August 1993

HERBARIUM BOGORIENSE HARVARD UNIVERSITY HERBARIA

\section{PLANTS OF INDONESIA}

Cat. \# 4097

\section{Lycopodium sp.}

Det. By: McDonald 1993

Locality:

Kabaena; Kabaena,

Batu Sangia Peak, ca. $7 \mathrm{~km}$ W of Tangkeno

$5^{\circ} 16^{\prime} \mathrm{S} 121^{\circ} 54^{\prime} \mathrm{E}$ Alt: $1200-1250 \mathrm{~m}$

Habitat:

Rocky knob of Batu Sangia, vegetation herbaceous or of stunted trees; associates include Ficus,

Dracaena, Shefflera, Kalanchoe, Tradescantia, many orchids.

Field Char:

Epiphyte growing in dead wood, stems branching, yellow-orange, leaves green or turning yellowish orange.

McDonald \& Ismail 4097

3 August 1993

HERBARIUM BOGORIENSE HARVARD UNIVERSITY HERBARIA 


\section{PLANTS OF INDONESIA}

Cat. \# 4098

Lycopodium sp.

Det. By: McDonald 1993

Locality:

Kabaena; Kabaena,

Batu Sangia Peak, ca. $7 \mathrm{~km} \mathrm{~W}$ of Tangkeno

$5^{\circ} 16^{\prime} \mathrm{S} 121^{\circ} 54^{\prime} \mathrm{E}$ Alt: $1200-1250 \mathrm{~m}$

Habitat:

Rocky knob of Batu Sangia, vegetation herbaceous

or of stunted trees; associates include Ficus,

Dracaena, Shefflera, Kalanchoe, Tradescantia, many orchids.

Field Char:

Branching herb, leaves green, sporangia yellow, growing on a decomposed log.

McDonald \& Ismail 4098

3 August 1993

\section{HERBARIUM BOGORIENSE \\ HARVARD UNIVERSITY HERBARIA}

\section{PLANTS OF INDONESIA}

Cat. \# 4099

\section{Elatostemma}

Det. By: McDonald 1993

Locality:

Kabaena; Kabaena,

Batu Sangia Peak, ca. $7 \mathrm{~km} \mathrm{~W}$ of Tangkeno

$5^{\circ} 16^{\prime} \mathrm{S} 121^{\circ} 54^{\prime} \mathrm{E}$ Alt: $1200-1250 \mathrm{~m}$

Habitat:

Rocky knob of Batu Sangia, vegetation herbaceous

or of stunted trees; associates include Ficus,

Dracaena, Shefflera, Kalanchoe, Tradescantia, many orchids.

Field Char:

Common herb, succulent, stems pale purple, leaves green above, pale green below, petioles purplish, inflorescence purplish.

McDonald \& Ismail 4099

3 August 1993

HERBARIUM BOGORIENSE HARVARD UNIVERSITY HERBARIA 


\section{PLANTS OF INDONESIA}

Cat. \# 4100

\section{Shefflera sp.}

Det. By: McDonald 1993

Locality:

Kabaena; Kabaena,

Batu Sangia Peak, ca. $7 \mathrm{~km} \mathrm{~W}$ of Tangkeno

$5^{\circ} 16^{\prime} \mathrm{S} 121^{\circ} 54^{\prime} \mathrm{E}$ Alt: $1200-1250 \mathrm{~m}$

Habitat:

Rocky knob of Batu Sangia, vegetation herbaceous or of stunted trees; associates include Ficus,

Dracaena, Shefflera, Kalanchoe, Tradescantia, many orchids.

Field Char:

Common, short shrubby tree, $2-5 \mathrm{~m}$ tall, branching above and below; leaves coriaceous, green above, pale green below; new stem growth green; sterile.

McDonald \& Ismail 4100

3 August 1993

\section{HERBARIUM BOGORIENSE \\ HARVARD UNIVERSITY HERBARIA}

\section{PLANTS OF INDONESIA}

Asteraceae

Cat. \# 4101

Gynura procumbens (Lour.) Merr.

Det. By: McDonald 1993

Locality:

Kabaena; Kabaena,

Batu Sangia Peak, ca. $7 \mathrm{~km} \mathrm{~W}$ of Tangkeno

$5^{\circ} 16^{\prime} \mathrm{S} 121^{\circ} 54^{\prime} \mathrm{E}$ Alt: $1200-1250 \mathrm{~m}$

Habitat:

Rocky knob of Batu Sangia, vegetation herbaceous

or of stunted trees; associates include Ficus,

Dracaena, Shefflera, Kalanchoe, Tradescantia, many orchids.

Field Char:

Occasional branching, sprawling, shrubby vine; stems green; leaves somewhat succulent, green, phyllaries light green or purple, corollas orange turning purple; pappus bright white. 


\section{PLANTS OF INDONESIA}

Euphorbiaceae

Cat. \# 4102

Breynia vitis-idaea (Burm) Fisch

Det. By: McDonald 1993

Locality:

Kabaena; Kabaena,

Batu Sangia Peak, ca. $7 \mathrm{~km} \mathrm{~W}$ of Tangkeno

$5^{\circ} 16^{\prime} \mathrm{S} 121^{\circ} 54^{\prime} \mathrm{E}$ Alt: $1200-1250 \mathrm{~m}$

Habitat:

Rocky knob of Batu Sangia, vegetation herbaceous or of stunted trees; associates include Ficus,

Dracaena, Shefflera, Kalanchoe, Tradescantia, many orchids.

Field Char:

Occasional short tree, $4 \mathrm{~m}$ tall, branching above; trunk cylindrical, crooked, $7 \mathrm{~cm}$ diam; bark tannish-

white; leaves green above, somewhat glaucous below; calyx pale green turning bright pink; fruits bright pink, turning dark purple.

McDonald \& Ismail 4102

3 August 1993

\section{HERBARIUM BOGORIENSE HARVARD UNIVERSITY HERBARIA}

\section{PLANTS OF INDONESIA}

Boraginaceae

Cat. \# 4103

Carmona retusas (Val.) Mus.

Det. By: McDonald 1993

Locality:

Kabaena; Kabaena,

Batu Sangia Peak, ca. $7 \mathrm{~km} \mathrm{~W}$ of Tangkeno

$5^{\circ} 16$ 'S $121^{\circ} 54^{\prime}$ 'E Alt: $1200-1250 \mathrm{~m}$

Habitat:

Rocky knob of Batu Sangia, vegetation herbaceous

or of stunted trees; associates include Ficus,

Dracaena, Shefflera, Kalanchoe, Tradescantia, many orchids.

Field Char:

Common shrub 1-2 m tall, branching much, sterile.

McDonald \& Ismail 4103

3 August 1993

HERBARIUM BOGORIENSE HARVARD UNIVERSITY HERBARIA 


\section{PLANTS OF INDONESIA}

Urticaceae

Cat. \# 4104

Pilea aff. calcarea Ridl.

Det. By: McDonald 1993

Locality:

Kabaena; Kabaena,

Batu Sangia Peak, ca. $7 \mathrm{~km} \mathrm{~W}$ of Tangkeno

$5^{\circ} 16^{\prime} \mathrm{S} 121^{\circ} 54^{\prime}$ E Alt: $1200-1250 \mathrm{~m}$

Habitat:

Rocky knob of Batu Sangia, vegetation herbaceous

or of stunted trees; associates include Ficus,

Dracaena, Shefflera, Kalanchoe, Tradescantia, many orchids.

Field Char:

Common erect herb in shady crevices, $1-1.5 \mathrm{~m}$ tall;

stems succulent; leaves glossy green; anthers white.

McDonald \& Ismail 4104

3 August 1993

\section{HERBARIUM BOGORIENSE HARVARD UNIVERSITY HERBARIA}

\section{PLANTS OF INDONESIA}

Rutaceae

Cat. \# 4105

Clausena excavata Burm. $f$.

Det. By: McDonald 1993

Locality:

Kabaena; Kabaena,

Batu Sangia Peak, ca. $7 \mathrm{~km} \mathrm{~W}$ of Tangkeno

$5^{\circ} 16^{\prime} \mathrm{S} 121^{\circ} 54^{\prime} \mathrm{E}$ Alt: $1200-1250 \mathrm{~m}$

Habitat:

Rocky knob of Batu Sangia, vegetation herbaceous

or of stunted trees; associates include Ficus,

Dracaena, Shefflera, Kalanchoe, Tradescantia, many orchids.

Field Char:

Forest margin canopy tree, $15 \mathrm{~m}$ tall, branching above; flowers ca. $1 \mathrm{~cm}$ diam during anthesis, petals ( sepals?) 5, cream, filaments fleshy at base, cream, anthers yoke, ovary 4-lobed.

McDonald \& Ismail 4105

3 August 1993

HERBARIUM BOGORIENSE HARVARD UNIVERSITY HERBARIA 


\section{PLANTS OF INDONESIA}

Poaceae

Cat. \# 4106

Poaceae

Det. By: McDonald 1993

Locality:

Kabaena; Kabaena,

Batu Sangia Peak, ca. $7 \mathrm{~km} \mathrm{~W}$ of Tangkeno

$5^{\circ} 16^{\prime}$ 'S $121^{\circ} 54^{\prime}$ E Alt: $1200-1250 \mathrm{~m}$

Habitat:

Rocky knob of Batu Sangia, vegetation herbaceous

or of stunted trees; associates include Ficus,

Dracaena, Shefflera, Kalanchoe, Tradescantia, many orchids.

Field Char:

Common grass, now dry, $1 \mathrm{~m}$ tall, clumped.

McDonald \& Ismail 4106

3 August 1993

\section{HERBARIUM BOGORIENSE \\ HARVARD UNIVERSITY HERBARIA}

\section{PLANTS OF INDONESIA}

Poaceae

Cat. \# 4107

\section{Poaceae}

Det. By: McDonald 1993

Locality:

Kabaena; Kabaena,

Batu Sangia Peak, ca. $7 \mathrm{~km}$ W of Tangkeno

$5^{\circ} 16^{\prime} \mathrm{S} 121^{\circ} 54^{\prime} \mathrm{E}$ Alt: $1200-1250 \mathrm{~m}$

Habitat:

Rocky knob of Batu Sangia, vegetation herbaceous

or of stunted trees; associates include Ficus,

Dracaena, Shefflera, Kalanchoe, Tradescantia, many orchids.

Field Char:

Small grass $10 \mathrm{~cm}$ tall, common on rock faces.

McDonald \& Ismail 4107

3 August 1993

HERBARIUM BOGORIENSE

HARVARD UNIVERSITY HERBARIA 


\section{PLANTS OF INDONESIA}

Begoniaceae

Cat. \# 4108

Begonia sp. nov.?

Det. By: McDonald 1993

Locality:

Kabaena; Kabaena,

Batu Sangia Peak, ca. $7 \mathrm{~km} \mathrm{~W}$ of Tangkeno

$5^{\circ} 16^{\prime} \mathrm{S} 121^{\circ} 54^{\prime} \mathrm{E}$ Alt: $1200-1250 \mathrm{~m}$

Habitat:

Rocky knob of Batu Sangia, vegetation herbaceous

or of stunted trees; associates include Ficus,

Dracaena, Shefflera, Kalanchoe, Tradescantia, many orchids.

Field Char:

Rare herb growing from deep rock crevices, stems succulent, new growth bright reddish, turning brownish later; petioles reddish, lamina green or reddish above, reddish below; petals pink, anthers yellow; fruits red and succulent before drying to brown.

McDonald \& Ismail 4108

3 August 1993

\section{HERBARIUM BOGORIENSE HARVARD UNIVERSITY HERBARIA}

\section{PLANTS OF INDONESIA}

Poaceae

Cat. \# 4109

\section{Poaceae}

Det. By: McDonald 1993

Locality:

Kabaena; Kabaena,

Batu Sangia Peak, ca. $7 \mathrm{~km}$ W of Tangkeno

$5^{\circ} 16^{\prime} \mathrm{S} 121^{\circ} 54^{\prime} \mathrm{E}$ Alt: $1200-1250 \mathrm{~m}$

Habitat:

Rocky knob of Batu Sangia, vegetation herbaceous

or of stunted trees; associates include Ficus,

Dracaena, Shefflera, Kalanchoe, Tradescantia, many orchids.

Field Char:

Occasional grass on dry cliff faces, stoloniferous.

McDonald \& Ismail 4109

3 August 1993

HERBARIUM BOGORIENSE HARVARD UNIVERSITY HERBARIA 


\section{PLANTS OF INDONESIA}

Asteraceae

Cat. \# 4110

Gynura

Det. By: McDonald 1993

Locality:

Kabaena; Kabaena,

Batu Sangia Peak, ca. $7 \mathrm{~km} \mathrm{~W}$ of Tangkeno

$5^{\circ} 16^{\prime} \mathrm{S} 121^{\circ} 54^{\prime} \mathrm{E}$ Alt: $1200-1250 \mathrm{~m}$

Habitat:

Rocky knob of Batu Sangia, vegetation herbaceous

or of stunted trees; associates include Ficus,

Dracaena, Shefflera, Kalanchoe, Tradescantia, many orchids.

Field Char:

Rare shrub, $50 \mathrm{~cm}$ tall, leaves green above, pale green below, corollas yellow.

McDonald \& Ismail 4110

3 August 1993

\section{HERBARIUM BOGORIENSE \\ HARVARD UNIVERSITY HERBARIA}

\section{PLANTS OF INDONESIA}

Lamiaceae

Cat. \# 4111

\section{Lamiaceae}

Det. By: McDonald 1993

Locality:

Kabaena; Kabaena,

Batu Sangia Peak, ca. $7 \mathrm{~km}$ W of Tangkeno

$5^{\circ} 16^{\prime} \mathrm{S} 121^{\circ} 54^{\prime} \mathrm{E}$ Alt: $1200-1250 \mathrm{~m}$

Habitat:

Rocky knob of Batu Sangia, vegetation herbaceous

or of stunted trees; associates include Ficus,

Dracaena, Shefflera, Kalanchoe, Tradescantia, many orchids.

Field Char:

Rare herb $30 \mathrm{~cm}$ tall, leaves green above, pale green below, corola white, with minute purple dots inside lower limb segments.

McDonald \& Ismail 4111

3 August 1993

HERBARIUM BOGORIENSE HARVARD UNIVERSITY HERBARIA 


\section{PLANTS OF INDONESIA}

Acanthaceae

Cat. \# 4112

Hypoestes

Det. By: McDonald 1993

Locality:

Kabaena; Kabaena,

Batu Sangia Peak, ca. $7 \mathrm{~km}$ W of Tangkeno

$5^{\circ} 16^{\prime} \mathrm{S} 121^{\circ} 54^{\prime} \mathrm{E}$ Alt: $1200-1250 \mathrm{~m}$

Habitat:

Rocky knob of Batu Sangia, vegetation herbaceous or of stunted trees; associates include Ficus,

Dracaena, Shefflera, Kalanchoe, Tradescantia, many orchids.

Field Char:

Erect shrub 1-1.5 m tall, stems brittle, branching sparingly at base; leaves dark green above, pale silver-green below; calyx green, corolla white, somewhat lavender on upper surface of upper limb segment, rusty-dotted on upper surface of lower limb segment.

McDonald \& Ismail 4112

3 August 1993

\section{HERBARIUM BOGORIENSE HARVARD UNIVERSITY HERBARIA}

\section{PLANTS OF INDONESIA}

Ericaceae

Cat. \# 4113

Diplycosia aff. heterophylla $\mathrm{Bl}$.

Det. By: McDonald 1993

Locality:

Kabaena; Kabaena,

Batu Sangia Peak, ca. $7 \mathrm{~km}$ W of Tangkeno

$5^{\circ} 16^{\prime} \mathrm{S} 121^{\circ} 54^{\prime} \mathrm{E}$ Alt: $1200-1250 \mathrm{~m}$

Habitat:

Rocky knob of Batu Sangia, vegetation herbaceous or of stunted trees; associates include Ficus,

Dracaena, Shefflera, Kalanchoe, Tradescantia, many orchids.

Field Char:

Occasional, colonial shrub, $1 \mathrm{~m}$ tall, branching profusely; colony $6 \mathrm{~m}$ in diam; leaves green above, pale green below, coriaceous, petioles red; corolla campanulate-urceolate, pale green, coriaceous, margins of limb segments red, anthers yellowish. 


\section{PLANTS OF INDONESIA}

Lamiaceae

Cat. \# 4114

Lamiaceae

Pogostemon sp. aff. methoides Be.

Det. By: McDonald 1993

Locality:

Kabaena; Kabaena,

Batu Sangia Peak, ca. $7 \mathrm{~km} \mathrm{~W}$ of Tangkeno

$5^{\circ} 16^{\prime} \mathrm{S} 121^{\circ} 54^{\prime} \mathrm{E}$ Alt: $1200-1250 \mathrm{~m}$

Habitat:

Rocky knob of Batu Sangia, vegetation herbaceous

or of stunted trees; associates include Ficus,

Dracaena, Shefflera, Kalanchoe, Tradescantia, many orchids.

Field Char:

Common, short shrub in exposed rock faces, $30 \mathrm{~cm}$ tall, rotund, branching; calyx green, corolla white, stamen filaments lavender.

McDonald \& Ismail 4114

3 August 1993

HERBARIUM BOGORIENSE

HARVARD UNIVERSITY HERBARIA

\section{PLANTS OF INDONESIA}

Lamiaceae

Cat. \# 4115

Leucas flaccida $R$. Br.

Det. By: McDonald 1993

Locality:

Kabaena; Kabaena,

Batu Sangia Peak, ca. $7 \mathrm{~km} \mathrm{~W}$ of Tangkeno

$5^{\circ} 16^{\prime} \mathrm{S} 121^{\circ} 54^{\prime} \mathrm{E}$ Alt: $1200-1250 \mathrm{~m}$

Habitat:

Rocky knob of Batu Sangia, vegetation herbaceous

or of stunted trees; associates include Ficus,

Dracaena, Shefflera, Kalanchoe, Tradescantia, many orchids.

Field Char:

Common, basally lignescent herb, leaves green above; calyx green, corolla white.

McDonald \& Ismail 4115

3 August 1993

HERBARIUM BOGORIENSE HARVARD UNIVERSITY HERBARIA 


\section{PLANTS OF INDONESIA}

Moraceae

Cat. \# 4116

Ficus sp.

Det. By: McDonald 1993

Locality:

Kabaena; Kabaena,

Batu Sangia Peak, ca. $7 \mathrm{~km} \mathrm{~W}$ of Tangkeno

$5^{\circ} 16^{\prime} \mathrm{S} 121^{\circ} 54^{\prime} \mathrm{E}$ Alt: $1200-1250 \mathrm{~m}$

Habitat:

Rocky knob of Batu Sangia, vegetation herbaceous

or of stunted trees; associates include Ficus,

Dracaena, Shefflera, Kalanchoe, Tradescantia, many orchids.

Field Char:

Occasional, dwarfed, shrubby tree, $3 \mathrm{~m}$ tall, rotund, branching profusely; fruits orange with tan punctae.

McDonald \& Ismail 4116

3 August 1993

\section{HERBARIUM BOGORIENSE HARVARD UNIVERSITY HERBARIA}

\section{PLANTS OF INDONESIA}

Araceae

Cat. \# 4117

Philodendron sp.

Det. By: McDonald 1993

Locality:

Kabaena; Kabaena,

Batu Sangia Peak, ca. $7 \mathrm{~km}$ W of Tangkeno

$5^{\circ} 16^{\prime} \mathrm{S} 121^{\circ} 54^{\prime} \mathrm{E}$ Alt: $1200-1250 \mathrm{~m}$

Habitat:

Rocky knob of Batu Sangia, vegetation herbaceous or of stunted trees; associates include Ficus,

Dracaena, Shefflera, Kalanchoe, Tradescantia, many orchids.

Field Char:

Occasional epiphytic shrub, branching sparingly, stems $5 \mathrm{~m}$ long, leaves only at apex; inflorescence 34 per apex, cream; infructescence bluish green when immature.

McDonald \& Ismail 4117

3 August 1993

HERBARIUM BOGORIENSE HARVARD UNIVERSITY HERBARIA 


\section{PLANTS OF INDONESIA}

Acanthaceae

Cat. \# 4118

Dicliptera sp.

Det. By: McDonald 1993

Locality:

Kabaena; Kabaena,

Batu Sangia Peak, ca. $7 \mathrm{~km}$ W of Tangkeno

$5^{\circ} 16^{\prime} \mathrm{S} 121^{\circ} 54^{\prime} \mathrm{E}$ Alt: $1200-1250 \mathrm{~m}$

Habitat:

Rocky knob of Batu Sangia, vegetation herbaceous

or of stunted trees; associates include Ficus,

Dracaena, Shefflera, Kalanchoe, Tradescantia, many orchids.

Field Char:

Occasional herb deep in rocky ravines, 1-2 $\mathrm{m}$ tall; stems green, leaves green; bracts light green, foliaceous, corolla lavender with purple spotted pattern inside upper throat, stamens exserted in lower part of throat.

McDonald \& Ismail 4118

3 August 1993

\section{HERBARIUM BOGORIENSE HARVARD UNIVERSITY HERBARIA}

\section{PLANTS OF INDONESIA}

Acanthaceae

Cat. \# 4119

Hypoestes polythrysa Miq.

Det. By: McDonald 1993

Locality:

Kabaena; Kabaena,

Batu Sangia Peak, ca. $7 \mathrm{~km}$ W of Tangkeno

$5^{\circ} 16^{\prime} \mathrm{S} 121^{\circ} 54^{\prime} \mathrm{E}$ Alt: $1200-1250 \mathrm{~m}$

Habitat:

Rocky knob of Batu Sangia, vegetation herbaceous

or of stunted trees; associates include Ficus,

Dracaena, Shefflera, Kalanchoe, Tradescantia, many orchids.

Field Char:

Common herbaceous shrub, $1 \mathrm{~m}$ tall, branching

much, leaves dark green, corolla purplish.

McDonald \& Ismail 4119

3 August 1993

HERBARIUM BOGORIENSE HARVARD UNIVERSITY HERBARIA 


\section{PLANTS OF INDONESIA}

Cat. \# 4120

Ryssopterys timoriense (DC) Juss. timoriense

Det. By: McDonald 1993

Locality:

Kabaena; Kabaena,

Batu Sangia Peak, ca. $7 \mathrm{~km} \mathrm{~W}$ of Tangkeno

$5^{\circ} 16^{\prime} \mathrm{S} 121^{\circ} 54^{\prime} \mathrm{E}$ Alt: $1200-1250 \mathrm{~m}$

Habitat:

Rocky knob of Batu Sangia, vegetation herbaceous or of stunted trees; associates include Ficus,

Dracaena, Shefflera, Kalanchoe, Tradescantia, many orchids.

Field Char:

Twining and climbing or scandent vine, stems branching; leaves green above, pale green below; calyx pale green, all other parts of flower bright yellow.

McDonald \& Ismail 4120

3 August 1993

HERBARIUM BOGORIENSE

HARVARD UNIVERSITY HERBARIA

\section{PLANTS OF INDONESIA}

Orchidaceae

Cat. \# 4121

Eria sp.

Det. By: McDonald 1993

Locality:

Kabaena; Kabaena,

Batu Sangia Peak, ca. $7 \mathrm{~km} \mathrm{~W}$ of Tangkeno

$5^{\circ} 16^{\prime} \mathrm{S} 121^{\circ} 54^{\prime} \mathrm{E}$ Alt: $1200-1250 \mathrm{~m}$

Habitat:

Rocky knob of Batu Sangia, vegetation herbaceous

or of stunted trees; associates include Ficus,

Dracaena, Shefflera, Kalanchoe, Tradescantia, many orchids.

Field Char:

Occasional epiphyte, sterile, foliage light green.

McDonald \& Ismail 4121

3 August 1993

HERBARIUM BOGORIENSE

HARVARD UNIVERSITY HERBARIA 


\section{PLANTS OF INDONESIA}

Solanaceae

Cat. \# 4122

Solanum lasiocarpum Dun.

Det. By: McDonald 1993

Locality:

Kabaena; Kabaena,

Batu Sangia Peak, ca. $7 \mathrm{~km} \mathrm{~W}$ of Tangkeno

$5^{\circ} 16^{\prime} \mathrm{S} 121^{\circ} 54^{\prime} \mathrm{E}$ Alt: $1200-1250 \mathrm{~m}$

Habitat:

Rocky knob of Batu Sangia, vegetation herbaceous or of stunted trees; associates include Ficus,

Dracaena, Shefflera, Kalanchoe, Tradescantia, many orchids.

Field Char:

Rare shrub, $1 \mathrm{~m}$ tall, branching, heavily armed; leaves dark breen above, pale green below; fruits imature, very pale green, pubescent.

McDonald \& Ismail $4122 \quad 3$ August 1993

HERBARIUM BOGORIENSE

HARVARD UNIVERSITY HERBARIA

\section{PLANTS OF INDONESIA}

Asteraceae

Cat. \# 4123

Ageratum conyzoides $L$.

Det. By: McDonald 1993

Locality:

Kabaena; Kabaena,

Batu Sangia Peak, ca. $7 \mathrm{~km}$ W of Tangkeno

$5^{\circ} 16^{\prime} \mathrm{S} 121^{\circ} 54^{\prime} \mathrm{E}$ Alt: $1200-1250 \mathrm{~m}$

Habitat:

Rocky knob of Batu Sangia, vegetation herbaceous

or of stunted trees; associates include Ficus,

Dracaena, Shefflera, Kalanchoe, Tradescantia, many orchids.

Field Char:

Rare weedy herb, branching, $30 \mathrm{~cm}$ tall; flowers

lavender.

McDonald \& Ismail 4123

3 August 1993

HERBARIUM BOGORIENSE HARVARD UNIVERSITY HERBARIA 


\section{PLANTS OF INDONESIA}

Balsaminaceae

Cat. \# 4124

Impatiens playpetala Lindl. ssp. aurantiaca (Teyxm.)Steen

Det. By: McDonald 1993

Locality:

Kabaena; Kabaena,

Batu Sangia Peak, ca. $7 \mathrm{~km}$ W of Tangkeno

$5^{\circ} 16^{\prime} \mathrm{S} 121^{\circ} 54^{\prime} \mathrm{E}$ Alt: $1200-1250 \mathrm{~m}$

Habitat:

Rocky knob of Batu Sangia, vegetation herbaceous

or of stunted trees; associates include Ficus,

Dracaena, Shefflera, Kalanchoe, Tradescantia, many orchids.

Field Char:

Rare herb $30 \mathrm{~cm}$ tall, branching, stems succulent, reddish-green, leaves green, corolla orange, spur arcuate, whitish.

McDonald \& Ismail 4124

3 August 1993

HERBARIUM BOGORIENSE

HARVARD UNIVERSITY HERBARIA

\section{PLANTS OF INDONESIA}

Cat. \# 4125

Pleomele angustifolia (Roxb.) Br.

Det. By: McDonald 1993

Locality:

Kabaena; Kabaena,

Batu Sangia Peak, ca. $7 \mathrm{~km} \mathrm{~W}$ of Tangkeno

$5^{\circ} 16^{\prime} \mathrm{S} 121^{\circ} 54^{\prime} \mathrm{E}$ Alt: $1200-1250 \mathrm{~m}$

Habitat:

Rocky knob of Batu Sangia, vegetation herbaceous

or of stunted trees; associates include Ficus,

Dracaena, Shefflera, Kalanchoe, Tradescantia, many orchids.

Field Char:

Branching shrub, $4 \mathrm{~m}$ tall, stems to $3 \mathrm{~cm}$ diam; leaves dark green above, pale green below, fruit immature, green.

McDonald \& Ismail 4125

3 August 1993

HERBARIUM BOGORIENSE HARVARD UNIVERSITY HERBARIA 


\section{PLANTS OF INDONESIA}

Cat. \# 4126

Cheilanthes sp.

Det. By:

Locality:

Kabaena; Kabaena,

Batu Sangia Peak, ca. $7 \mathrm{~km} \mathrm{~W}$ of Tangkeno

$5^{\circ} 16^{\prime} \mathrm{S} 121^{\circ} 54^{\prime} \mathrm{E}$ Alt: $1200-1250 \mathrm{~m}$

Habitat:

Rocky knob of Batu Sangia, vegetation herbaceous

or of stunted trees; associates include Ficus,

Dracaena, Shefflera, Kalanchoe, Tradescantia, many orchids.

Field Char:

Colonial, leaves green above, white below.

McDonald \& Ismail 4126

3 August 1993

\section{HERBARIUM BOGORIENSE HARVARD UNIVERSITY HERBARIA}

\section{PLANTS OF INDONESIA}

Cat. \# 4127

Athyrium sp.

Det. By:

Locality:

Kabaena; Kabaena,

Batu Sangia Peak, ca. $7 \mathrm{~km} \mathrm{~W}$ of Tangkeno

$5^{\circ} 16^{\prime} \mathrm{S} 121^{\circ} 54^{\prime} \mathrm{E}$ Alt: $1200-1250 \mathrm{~m}$

Habitat:

Rocky knob of Batu Sangia, vegetation herbaceous

or of stunted trees; associates include Ficus,

Dracaena, Shefflera, Kalanchoe, Tradescantia, many orchids.

Field Char:

Common, creeping, stoloniferous; stems light green, hairs brown; frond light green with white punctae on upper surface.

McDonald \& Ismail 4127

3 August 1993

HERBARIUM BOGORIENSE HARVARD UNIVERSITY HERBARIA 


\section{PLANTS OF INDONESIA}

Cat. \# 4128

Fern

Det. By:

Locality:

Kabaena; Kabaena,

Batu Sangia Peak, ca. $7 \mathrm{~km}$ W of Tangkeno

$5^{\circ} 16^{\prime} \mathrm{S} 121^{\circ} 54^{\prime} \mathrm{E}$ Alt: $1200-1250 \mathrm{~m}$

Habitat:

Rocky knob of Batu Sangia, vegetation herbaceous or of stunted trees; associates include Ficus,

Dracaena, Shefflera, Kalanchoe, Tradescantia, many orchids.

Field Char:

Common creeping, stoloniferous fern; stems brown, hairy; fronds light green.

McDonald \& Ismail 4128

3 August 1993

\section{HERBARIUM BOGORIENSE \\ HARVARD UNIVERSITY HERBARIA}

\section{PLANTS OF INDONESIA}

Cat. \# 4129

Fern

Det. By:

Locality:

Kabaena; Kabaena,

Batu Sangia Peak, ca. $7 \mathrm{~km}$ W of Tangkeno

$5^{\circ} 16^{\prime} \mathrm{S} 121^{\circ} 54^{\prime} \mathrm{E}$ Alt: $1200-1250 \mathrm{~m}$

Habitat:

Rocky knob of Batu Sangia, vegetation herbaceous or of stunted trees; associates include Ficus,

Dracaena, Shefflera, Kalanchoe, Tradescantia, many orchids.

Field Char:

Prostrate fern, branching, rooting on rocks; stems brown, roots black, leaves coriaceous, yellow-green; fertile fronds narrow.

McDonald \& Ismail 4129

3 August 1993

HERBARIUM BOGORIENSE HARVARD UNIVERSITY HERBARIA 


\section{PLANTS OF INDONESIA}

Celastraceae

Cat. \# 4130

Euonymus javanicus $\mathbf{B l}$.

Det. By: McDonald 1993

Locality:

Kabaena; Kabaena,

Batu Sangia Peak, ca. $7 \mathrm{~km} \mathrm{~W}$ of Tangkeno

$5^{\circ} 16^{\prime} \mathrm{S} 121^{\circ} 54^{\prime}$ 'E Alt: $1200-1250 \mathrm{~m}$

Habitat:

Rocky knob of Batu Sangia, vegetation herbaceous

or of stunted trees; associates include Ficus,

Dracaena, Shefflera, Kalanchoe, Tradescantia, many orchids.

Field Char:

Tree $7 \mathrm{~m}$ tall, branching above, trunk cylindrical, 7

$\mathrm{cm}$ diam; fruits immature, green.

McDonald \& Ismail 4130

3 August 1993

\section{HERBARIUM BOGORIENSE \\ HARVARD UNIVERSITY HERBARIA}

\section{PLANTS OF INDONESIA}

Agavaceae

Cat. \# 4131

Dracaena sp.

Det. By: McDonald 1993

Locality:

Kabaena; Kabaena,

Batu Sangia Peak, ca. $7 \mathrm{~km}$ W of Tangkeno

$5^{\circ} 16^{\prime} \mathrm{S} 121^{\circ} 54^{\prime} \mathrm{E}$ Alt: $1200-1250 \mathrm{~m}$

Habitat:

Rocky knob of Batu Sangia, vegetation herbaceous

or of stunted trees; associates include Ficus,

Dracaena, Shefflera, Kalanchoe, Tradescantia, many orchids.

Field Char:

Common tree $12 \mathrm{~m}$ tall, branching above,

dichotomous; stems thick, $>4 \mathrm{~cm}$ diam;

inflorescence old, fruits lacking.

McDonald \& Ismail 4131

3 August 1993

HERBARIUM BOGORIENSE HARVARD UNIVERSITY HERBARIA 


\section{PLANTS OF INDONESIA}

Orchidaceae

Cat. \# 4132

Orchidaceae

Det. By: McDonald 1993

Locality:

Kabaena; Kabaena,

Batu Sangia Peak, ca. $7 \mathrm{~km} \mathrm{~W}$ of Tangkeno

$5^{\circ} 16^{\prime} \mathrm{S} 121^{\circ} 54^{\prime} \mathrm{E}$ Alt: $1200-1250 \mathrm{~m}$

Habitat:

Rocky knob of Batu Sangia, vegetation herbaceous

or of stunted trees; associates include Ficus,

Dracaena, Shefflera, Kalanchoe, Tradescantia, many orchids.

Field Char:

Common solitary orchid; bulbil in cracks, succulent, corolla tubular, white.

McDonald \& Ismail 4132

3 August 1993

\section{HERBARIUM BOGORIENSE \\ HARVARD UNIVERSITY HERBARIA}

\section{PLANTS OF INDONESIA}

Cat. \# 4133

Elatostemma?

Det. By: McDonald 1993

Locality:

Kabaena; Kabaena,

Batu Sangia Peak, ca. $7 \mathrm{~km} \mathrm{~W}$ of Tangkeno

$5^{\circ} 16^{\prime} \mathrm{S} 121^{\circ} 54^{\prime} \mathrm{E}$ Alt: $1200-1250 \mathrm{~m}$

Habitat:

Rocky knob of Batu Sangia, vegetation herbaceous

or of stunted trees; associates include Ficus,

Dracaena, Shefflera, Kalanchoe, Tradescantia, many orchids.

Field Char:

Common herbaceous succulent, $10-20 \mathrm{~cm}$ tall; leaves green.

McDonald \& Ismail 4133

3 August 1993

HERBARIUM BOGORIENSE

HARVARD UNIVERSITY HERBARIA 


\section{PLANTS OF INDONESIA}

Asclepiadaceae

Cat. \# 4134

Dischidia sp.

Det. By: McDonald 1993

Locality:

Kabaena; Kabaena,

Batu Sangia Peak, ca. $7 \mathrm{~km}$ W of Tangkeno

$5^{\circ} 16^{\prime} \mathrm{S} 121^{\circ} 54^{\prime} \mathrm{E}$ Alt: $1200-1250 \mathrm{~m}$

Habitat:

Rocky knob of Batu Sangia, vegetation herbaceous

or of stunted trees; associates include Ficus,

Dracaena, Shefflera, Kalanchoe, Tradescantia, many orchids.

Field Char:

Common scandent vine, stems to $10 \mathrm{~m}$ long,

branching, succulent, palid green.

McDonald \& Ismail 4134

3 August 1993

\section{HERBARIUM BOGORIENSE \\ HARVARD UNIVERSITY HERBARIA}

\section{PLANTS OF INDONESIA}

Convolvulaceae

Cat. \# 4135

Ipomoea digitata $L . \quad$ var. eriosperma (P.B.) Rendl

Det. By: McDonald 1993

Locality:

Kabaena; Kabaena,

Batu Sangia Peak, ca. $7 \mathrm{~km} \mathrm{~W}$ of Tangkeno

$5^{\circ} 16^{\prime} \mathrm{S} 121^{\circ} 54^{\prime} \mathrm{E}$ Alt: $1200-1250 \mathrm{~m}$

Habitat:

Rocky knob of Batu Sangia, vegetation herbaceous

or of stunted trees; associates include Ficus,

Dracaena, Shefflera, Kalanchoe, Tradescantia, many orchids.

Field Char:

Dried up lianas, fruits and seeds persistent, brown, dry.

McDonald \& Ismail 4135

3 August 1993

HERBARIUM BOGORIENSE HARVARD UNIVERSITY HERBARIA 


\section{PLANTS OF INDONESIA}

Gesneriaceae

Cat. \# 4136

Aeschynanthus sp.

Det. By: McDonald 1993

Locality:

Kabaena; Kabaena,

Batu Sangia Peak, ca. $7 \mathrm{~km}$ W of Tangkeno

$5^{\circ} 16^{\prime} \mathrm{S} 121^{\circ} 54^{\prime} \mathrm{E}$ Alt: $1200-1250 \mathrm{~m}$

Habitat:

Rocky knob of Batu Sangia, vegetation herbaceous

or of stunted trees; associates include Ficus,

Dracaena, Shefflera, Kalanchoe, Tradescantia, many orchids.

Field Char:

Succulent epiphyte on trees and rocks, leaves succulent, calyx maroon, corolla reddish.

McDonald \& Ismail 4136

3 August 1993

\section{HERBARIUM BOGORIENSE \\ HARVARD UNIVERSITY HERBARIA}

\section{PLANTS OF INDONESIA}

Asteraceae

Cat. \# 4137

Blumea riparia (Bl.) $D C$.

Det. By: McDonald 1993

Locality:

Kabaena; Kabaena,

Batu Sangia Peak, ca. $7 \mathrm{~km}$ W of Tangkeno

$5^{\circ} 16^{\prime} \mathrm{S} 121^{\circ} 54^{\prime} \mathrm{E}$ Alt: $1200-1250 \mathrm{~m}$

Habitat:

Rocky knob of Batu Sangia, vegetation herbaceous

or of stunted trees; associates include Ficus,

Dracaena, Shefflera, Kalanchoe, Tradescantia, many orchids.

Field Char:

Branching, shrubby vine, stems and leaves green; phyllaries pale green, corolla yellow.

McDonald \& Ismail 4137

3 August 1993

HERBARIUM BOGORIENSE HARVARD UNIVERSITY HERBARIA 


\section{PLANTS OF INDONESIA}

Orchidaceae

Cat. \# 4138

Eria sp.

Det. By: McDonald 1993

Locality:

Kabaena; Kabaena,

Batu Sangia Peak, ca. $7 \mathrm{~km} \mathrm{~W}$ of Tangkeno

$5^{\circ} 16^{\prime} \mathrm{S} 121^{\circ} 54^{\prime} \mathrm{E}$ Alt: $1200-1250 \mathrm{~m}$

Habitat:

Rocky knob of Batu Sangia, vegetation herbaceous

or of stunted trees; associates include Ficus,

Dracaena, Shefflera, Kalanchoe, Tradescantia, many orchids.

Field Char:

Understory, terrestrial orchid, leaves very pale green below, green above; bracts pale pink, ovary bright

green, outer tepals green, inner cream.

McDonald \& Ismail 4138

3 August 1993

\section{HERBARIUM BOGORIENSE \\ HARVARD UNIVERSITY HERBARIA}

\section{PLANTS OF INDONESIA}

Sapindaceae

Cat. \# 4139

Lepisanthes $s p$.

Det. By: McDonald 1993

Locality:

Kabaena; Kabaena,

Batu Sangia Peak, ca. $7 \mathrm{~km} \mathrm{~W}$ of Tangkeno

$5^{\circ} 16^{\prime} \mathrm{S} 121^{\circ} 54^{\prime} \mathrm{E}$ Alt: $1200-1250 \mathrm{~m}$

Habitat:

Rocky knob of Batu Sangia, vegetation herbaceous

or of stunted trees; associates include Ficus,

Dracaena, Shefflera, Kalanchoe, Tradescantia, many orchids.

Field Char:

Common understory tree, $5 \mathrm{~m}$ tall, branching above; leaves green above, light green below, petals deep maroon, filaments white, anthers dull yellow.

McDonald \& Ismail 4139

3 August 1993

HERBARIUM BOGORIENSE HARVARD UNIVERSITY HERBARIA 


\section{PLANTS OF INDONESIA}

Convolvulaceae

Cat. \# 4140

Merremia umbellata (L.) Hall. $f$.

Det. By: McDonald 1993

Locality:

Kabaena; Kabaena,

Ridge between Tangkeno and Batu Sangia, 1-3 km

$\mathrm{W}$ of Tangkeno.

$5^{\circ} 16^{\prime} \mathrm{S} 121^{\circ} 56^{\prime} \mathrm{E}$ Alt: $700-800 \mathrm{~m}$

Habitat:

Secondary forest around ladangs; associates include

Arenga, Ficus, Trichospermum, Albizia, baboos, etc.

Field Char:

Slender vine, stem $5 \mathrm{~m}$ long, twining, calyx pale green, tinged purple at apex, corolla white; anthers recurved.

McDonald \& Ismail 4140

3 August 1993

\section{HERBARIUM BOGORIENSE \\ HARVARD UNIVERSITY HERBARIA}

\section{PLANTS OF INDONESIA}

Convolvulaceae

Cat. \# 4141

Merremia vitifolia (Burm.) Hall.

Det. By: McDonald 1993

Locality:

Kabaena; Kabaena,

Ridge between Tangkeno and Batu Sangia, 1-3 km

$\mathrm{W}$ of Tangkeno.

$5^{\circ} 16^{\prime} \mathrm{S} 121^{\circ} 56^{\prime} \mathrm{E}$ Alt: $700-800 \mathrm{~m}$

Habitat:

Secondary forest around ladangs; associates include

Arenga, Ficus, Trichospermum, Albizia, baboos, etc.

Field Char:

Twining, robust vine, stems $6 \mathrm{~m}$ long, twining, green, tipped purple, corolla yellow, anthers cream, recurved; fruit breaking irregularly.

McDonald \& Ismail 4141

3 August 1993

HERBARIUM BOGORIENSE HARVARD UNIVERSITY HERBARIA 


\section{PLANTS OF INDONESIA}

Convolvulaceae

Cat. \# 4142

Ipomoea digitata $L$. var. eriosperma (P.B.) Rendl

Det. By: McDonald 1993

Locality:

Kabaena; Kabaena,

Ridge between Tangkeno and Batu Sangia, 1-3 km

W of Tangkeno.

$5^{\circ} 16^{\prime} \mathrm{S} 121^{\circ} 56^{\prime} \mathrm{E}$ Alt: $700-800 \mathrm{~m}$

Habitat:

Secondary forest around ladangs; associates include

Arenga, Ficus, Trichospermum, Albizia, baboos, etc.

Field Char:

Liana, stems $6 \mathrm{~m}$ long, branching, green; leaves

green and glossy above; calyx pale green, corolla

lavender, stamens white.

McDonald \& Ismail 4142

3 August 1993

HERBARIUM BOGORIENSE

HARVARD UNIVERSITY HERBARIA

\section{PLANTS OF INDONESIA}

Orchidaceae

Cat. \# 4143

\section{Orchidaceae}

Det. By: McDonald 1993

Locality:

Kabaena; Kabaena,

Summit of Sangia Wita, ca. $10 \mathrm{~km}$ SE of Tangkeno.

$5^{\circ} 19^{\prime} \mathrm{S} 121^{\circ} 58^{\prime} \mathrm{E}$ Alt: $1500 \mathrm{~m}$

Habitat:

Short, sclerophyllous, subelfin forest; associates

include Melastoma, Actinodaphne, Meliosma,

Calamus, Daphniphyllum; soils serpentine.

Field Char:

Epiphyte on mossy tree branches, common, succulent, mature plants light green; inflorescence young, yellow green.

McDonald \& Ismail 4143

5 August 1993

HERBARIUM BOGORIENSE

HARVARD UNIVERSITY HERBARIA 


\section{PLANTS OF INDONESIA}

Orchidaceae

Cat. \# 4144

Dendrobium macrophyllum A. Rich

Det. By: McDonald 1993

Locality:

Kabaena; Kabaena,

Summit of Sangia Wita, ca. $10 \mathrm{~km}$ SE of Tangkeno. $5^{\circ} 19^{\prime} \mathrm{S} 121^{\circ} 58^{\prime} \mathrm{E}$ Alt: $1500 \mathrm{~m}$

Habitat:

Short, sclerophyllous, subelfin forest; associates include Melastoma, Actinodaphne, Meliosma, Calamus, Daphniphyllum; soils serpentine.

Field Char:

Common epiphyte on mossy branches, bulbil purple striate after fruition, green, smooth during anthesis; flowers 3 , ovary long-hairy, greenish, outer tepals 5 , yellow, setose and spotted purple outside, labellum greenish, striate-purple above, mottled-spotted purple distally.

McDonald \& Ismail 4144

5 August 1993

\section{HERBARIUM BOGORIENSE HARVARD UNIVERSITY HERBARIA}

\section{PLANTS OF INDONESIA}

Orchidaceae

Cat. \# 4145

\section{Orchidaceae}

Det. By: McDonald 1993

Locality:

Kabaena; Kabaena,

Summit of Sangia Wita, ca. $10 \mathrm{~km}$ SE of Tangkeno.

$5^{\circ} 19^{\prime} \mathrm{S} 121^{\circ} 58^{\prime} \mathrm{E}$ Alt: $1500 \mathrm{~m}$

Habitat:

Short, sclerophyllous, subelfin forest; associates include Melastoma, Actinodaphne, Meliosma,

Calamus, Daphniphyllum; soils serpentine.

Field Char:

Rare, monopodial orchid, epiphytic, $40 \mathrm{~cm}$ long,

lamina elliptic, $7 \mathrm{~cm}$ long.

McDonald \& Ismail 4145

5 August 1993

HERBARIUM BOGORIENSE HARVARD UNIVERSITY HERBARIA 


\title{
PLANTS OF INDONESIA
}

Zingiberaceae

Cat. \# 4146

Alpinia sp. nov. McDonald ined.

Det. By: McDonald 1993

Locality:

Kabaena; Kabaena,

Summit of Sangia Wita, ca. $10 \mathrm{~km}$ SE of Tangkeno.

$5^{\circ} 19^{\prime} \mathrm{S} 121^{\circ} 58^{\prime} \mathrm{E}$ Alt: $1500 \mathrm{~m}$

Habitat:

Short, sclerophyllous, subelfin forest; associates

include Melastoma, Actinodaphne, Meliosma,

Calamus, Daphniphyllum; soils serpentine.

Field Char:

5-6 stems branching basally from rootstock, stems 1-

$2 \mathrm{~m}$ long; lamina green above, pale green below;

peduncle dark brown, calyx light green, corolla

fleshy, whitish; fruits rotund with apical, round beak.

McDonald \& Ismail 4146

5 August 1993

\section{HERBARIUM BOGORIENSE \\ HARVARD UNIVERSTTY HERBARIA}

\section{PLANTS OF INDONESIA}

Arecaceae

Cat. \# 4147

Calamus sp.

Det. By: McDonald 1993

Locality:

Kabaena; Kabaena,

Summit of Sangia Wita, ca. $10 \mathrm{~km} \mathrm{SE}$ of Tangkeno.

$5^{\circ} 19^{\prime} \mathrm{S} 121^{\circ} 58^{\prime} \mathrm{E}$ Alt: $1500 \mathrm{~m}$

Habitat:

Short, sclerophyllous, subelfin forest; associates

include Melastoma, Actinodaphne, Meliosma,

Calamus, Daphniphyllum; soils serpentine.

Field Char:

Subdominant palm, 2-5 m tall, fronds 2-4 m diam, many bearing a long spiny leaf tip of equal length, fruits whitish, flowers pink.

McDonald \& Ismail 4147

5 August 1993

\author{
HERBARIUM BOGORIENSE \\ HARVARD UNIVERSITY HERBARIA
}




\section{PLANTS OF INDONESIA}

Cat. \# 4148

Fern

Det. By: McDonald 1993

Locality:

Kabaena; Kabaena,

Summit of Sangia Wita, ca. $10 \mathrm{~km}$ SE of Tangkeno. $5^{\circ} 19^{\prime} \mathrm{S} 121^{\circ} 58^{\prime} \mathrm{E}$ Alt: $1500 \mathrm{~m}$

Habitat:

Short, sclerophyllous, subelfin forest; associates include Melastoma, Actinodaphne, Meliosma, Calamus, Daphniphyllum; soils serpentine.

Field Char:

Stems reddish brown, petioles the same, lamina light green above, $\tan$ or brown below.

McDonald \& Ismail 4148

5 August 1993

HERBARIUM BOGORIENSE HARVARD UNIVERSITY HERBARIA

\section{PLANTS OF INDONESIA}

Asteraceae

Cat. \# 4149

Vernonia sp.

Det. By: McDonald 1993

Locality:

Kabaena; Kabaena,

Summit of Sangia Wita, ca. $10 \mathrm{~km}$ SE of Tangkeno.

$5^{\circ} 19^{\prime} \mathrm{S} 121^{\circ} 58^{\prime} \mathrm{E}$ Alt: $1500 \mathrm{~m}$

Habitat:

Short, sclerophyllous, subelfin forest; associates

include Melastoma, Actinodaphne, Meliosma,

Calamus, Daphniphyllum; soils serpentine.

Field Char:

Occasional shrub $1 \mathrm{~m}$ tall, root base embedded in rock; stems brittle; phyllaries green, pappus white, corolla tube white, segments purplish, style branches white.

McDonald \& Ismail 4149

5 August 1993

HERBARIUM BOGORIENSE HARVARD UNIVERSITY HERBARIA 


\section{PLANTS OF INDONESIA}

Loranthaceae

Cat. \# 4150

\section{Loranthaceae}

Det. By: McDonald 1993

Locality:

Kabaena; Kabaena,

Summit of Sangia Wita, ca. $10 \mathrm{~km}$ SE of Tangkeno.

$5^{\circ} 19^{\prime} \mathrm{S} 121^{\circ} 58^{\prime} \mathrm{E}$ Alt: $1500 \mathrm{~m}$

Habitat:

Short, sclerophyllous, subelfin forest; associates include Melastoma, Actinodaphne, Meliosma,

Calamus, Daphniphyllum; soils serpentine.

Field Char:

Rare parasite; stems woody, creeping along host stems; leaves coriaceous, green below, reddish purple above; fruits reddish and green.

McDonald \& Ismail 4150

5 August 1993

\section{HERBARIUM BOGORIENSE \\ HARVARD UNIVERSITY HERBARIA}

\section{PLANTS OF INDONESIA}

Lauraceae

Cat. \# 4151

Actinodaphne sp.

Det. By: McDonald 1993

Locality:

Kabaena; Kabaena,

Summit of Sangia Wita, ca. $10 \mathrm{~km}$ SE of Tangkeno.

$5^{\circ} 19^{\prime}$ S $121^{\circ} 58^{\prime} \mathrm{E}$ Alt: $1500 \mathrm{~m}$

Habitat:

Short, sclerophyllous, subelfin forest; associates include Melastoma, Actinodaphne, Meliosma,

Calamus, Daphniphyllum; soils serpentine.

Field Char:

Common shrub, round, $3 \mathrm{~m}$ diam, branching; leaves green above, glaucous below.

McDonald \& Ismail 4151

5 August 1993

HERBARIUM BOGORIENSE

HARVARD UNIVERSITY HERBARIA 


\section{PLANTS OF INDONESIA}

Ericaceae

Cat. \# 4152

Vaccinium kjellbergii J.J.S.

Det. By: McDonald 1993

Locality:

Kabaena; Kabaena,

Summit of Sangia Wita, ca. $10 \mathrm{~km}$ SE of Tangkeno.

$5^{\circ} 19^{\prime} \mathrm{S} 121^{\circ} 58^{\prime} \mathrm{E}$ Alt: $1500 \mathrm{~m}$

Habitat:

Short, sclerophyllous, subelfin forest; associates include Melastoma, Actinodaphne, Meliosma, Calamus, Daphniphyllum; soils serpentine.

Field Char:

Common rotund shrub, $1 \mathrm{~m}$ tall, branching profusely; leaves green above, light green below; calyx purplish, corolla cream.

McDonald \& Ismail 4152

5 August 1993

\section{HERBARIUM BOGORIENSE HARVARD UNIVERSITY HERBARIA}

\section{PLANTS OF INDONESIA}

Ericaceae

Cat. \# 4153

Vaccinium sp.

Det. By: McDonald 1993

Locality:

Kabaena; Kabaena,

Summit of Sangia Wita, ca. $10 \mathrm{~km}$ SE of Tangkeno.

$5^{\circ} 19^{\prime} \mathrm{S} 121^{\circ} 58^{\prime} \mathrm{E}$ Alt: $1500 \mathrm{~m}$

Habitat:

Short, sclerophyllous, subelfin forest; associates include Melastoma, Actinodaphne, Meliosma, Calamus, Daphniphyllum; soils serpentine.

Field Char:

Uncommon shrubby tree $3 \mathrm{~m}$ tall, branching profusely; leaves green above, light green below; corolla ca. $6 \mathrm{~mm}$ long, $3 \mathrm{~mm}$ diam, white; fruits rotund, green, ca. $6 \mathrm{~mm}$ diam.

McDonald \& Ismail 4153 


\section{PLANTS OF INDONESIA}

Acanthaceae

Cat. \# 4154

Justicia aff. blumeana Klutz

Det. By: McDonald 1993

Locality:

Kabaena; Kabaena,

Summit of Sangia Wita, ca. $10 \mathrm{~km}$ SE of Tangkeno.

$5^{\circ} 19^{\prime} \mathrm{S} 121^{\circ} 58^{\prime} \mathrm{E}$ Alt: $1500 \mathrm{~m}$

Habitat:

Short, sclerophyllous, subelfin forest; associates

include Melastoma, Actinodaphne, Meliosma,

Calamus, Daphniphyllum; soils serpentine.

Field Char:

Common, somewhat herbaceous shrub, $50-100 \mathrm{~cm}$ tall, branching much from base, leaves dark green, bracts palid green, corolla white, stippled pale-

lavender inside lower limb segment.

McDonald \& Ismail 4154

5 August 1993

HERBARIUM BOGORIENSE

HARVARD UNIVERSITY HERBARIA

\section{PLANTS OF INDONESIA}

Aquifoliaceae

Cat. \# 4155

Ilex

Det. By: McDonald 1993

Locality:

Kabaena; Kabaena,

Summit of Sangia Wita, ca. $10 \mathrm{~km} \mathrm{SE}$ of Tangkeno.

$5^{\circ} 19^{\prime} \mathrm{S} 121^{\circ} 58^{\prime} \mathrm{E}$ Alt: $1500 \mathrm{~m}$

Habitat:

Short, sclerophyllous, subelfin forest; associates

include Melastoma, Actinodaphne, Meliosma,

Calamus, Daphniphyllum; soils serpentine.

Field Char:

Occasional understory shrub, $1.5 \mathrm{~m}$ tall, branching above and below, leaves green above, pale green below, corolla white, pistil green.

McDonald \& Ismail 4155

5 August 1993

HERBARIUM BOGORIENSE HARVARD UNIVERSITY HERBARIA 


\section{PLANTS OF INDONESIA}

Asclepiadaceae

Cat. \# 4156

Asclepias

Det. By: McDonald 1993

Locality:

Kabaena; Kabaena,

Summit of Sangia Wita, ca. $10 \mathrm{~km} \mathrm{SE}$ of Tangkeno.

$5^{\circ} 19^{\prime} \mathrm{S} 121^{\circ} 58^{\prime} \mathrm{E}$ Alt: $1500 \mathrm{~m}$

Habitat:

Short, sclerophyllous, subelfin forest; associates include Melastoma, Actinodaphne, Meliosma, Calamus, Daphniphyllum; soils serpentine.

Field Char:

Occasional vine, stems twining, to $5 \mathrm{~m}$ long, calyx greenish, petal segments 5 , maroonish with two medial yellowish lines, corona campanulate, white; fruits purple.

McDonald \& Ismail 4156

5 August 1993

HERBARIUM BOGORIENSE

HARVARD UNIVERSITY HERBARIA

\section{PLANTS OF INDONESIA}

Rhamnaceae

Cat. \# 4157

Rhamnus nipalensis (Wall.) Laws.

Det. By: McDonald 1993

Locality:

Kabaena; Kabaena,

Summit of Sangia Wita, ca. $10 \mathrm{~km} \mathrm{SE}$ of Tangkeno.

$5^{\circ} 19^{\prime} \mathrm{S} 121^{\circ} 58^{\prime} \mathrm{E}$ Alt: $1500 \mathrm{~m}$

Habitat:

Short, sclerophyllous, subelfin forest; associates

include Melastoma, Actinodaphne, Meliosma,

Calamus, Daphniphyllum; soils serpentine.

Field Char:

Occasional shrub at summit (liana in lower forest), rotund, $2 \mathrm{~m}$ diam, $1 \mathrm{~m}$ tall, branching profusely, leaves dark green above, light green below, flowers green; fruit triangular-rotund, $7 \mathrm{~mm}$ diam, red.

McDonald \& Ismail 4157

5 August 1993

HERBARIUM BOGORIENSE HARVARD UNIVERSITY HERBARIA 


\section{PLANTS OF INDONESIA}

Symplocaceae

Cat. \# 4158

Symplocos sp.

Det. By: McDonald 1993

Locality:

Kabaena; Kabaena,

Summit of Sangia Wita, ca. $10 \mathrm{~km}$ SE of Tangkeno.

$5^{\circ} 19^{\prime} \mathrm{S} 121^{\circ} 58^{\prime} \mathrm{E}$ Alt: $1500 \mathrm{~m}$

Habitat:

Short, sclerophyllous, subelfin forest; associates

include Melastoma, Actinodaphne, Meliosma,

Calamus, Daphniphyllum; soils serpentine.

Field Char:

Common round shrub $1 \mathrm{~m}$ tall, $2 \mathrm{~m}$ diam, branching profusely, new growth stems and petioles rusty-

pubescent; fruits green.

McDonald \& Ismail 4158

5 August 1993

HERBARIUM BOGORIENSE

HARVARD UNIVERSITY HERBARIA

\section{PLANTS OF INDONESIA}

Poaceae

Cat. \# 4159

\section{Poaceae}

Det. By: McDonald 1993

Locality:

Kabaena; Kabaena,

Summit of Sangia Wita, ca. $10 \mathrm{~km}$ SE of Tangkeno.

$5^{\circ} 19^{\prime} \mathrm{S} 121^{\circ} 58^{\prime} \mathrm{E}$ Alt: $1500 \mathrm{~m}$

Habitat:

Short, sclerophyllous, subelfin forest; associates

include Melastoma, Actinodaphne, Meliosma,

Calamus, Daphniphyllum; soils serpentine.

Field Char:

Dominant grass in open areas; stems reddish, lamina

light green, fruits rare at this time.

McDonald \& Ismail 4159

5 August 1993

HERBARIUM BOGORIENSE

HARVARD UNIVERSITY HERBARIA 


\section{PLANTS OF INDONESIA}

Cyperaceae

Cat. \# 4160

Scleria scrobiculata Nees \& Mays

Det. By: McDonald 1993

Locality:

Kabaena; Kabaena,

Summit of Sangia Wita, ca. $10 \mathrm{~km}$ SE of Tangkeno.

$5^{\circ} 19^{\prime} \mathrm{S} 121^{\circ} 58^{\prime} \mathrm{E}$ Alt: $1500 \mathrm{~m}$

Habitat:

Short, sclerophyllous, subelfin forest; associates

include Melastoma, Actinodaphne, Meliosma,

Calamus, Daphniphyllum; soils serpentine.

Field Char:

Solitary or small clumps, 1-1.5 m tall, foliage light green, inflorescence somewhat orangish.

McDonald \& Ismail 4160

5 August 1993

HERBARIUM BOGORIENSE

HARVARD UNIVERSITY HERBARIA

\section{PLANTS OF INDONESIA}

Cat. \# 4161

\section{Andiandra}

Det. By: McDonald 1993

Locality:

Kabaena; Kabaena,

Summit of Sangia Wita, ca. $10 \mathrm{~km}$ SE of Tangkeno.

$5^{\circ} 19^{\prime} \mathrm{S} 121^{\circ} 58^{\prime} \mathrm{E}$ Alt: $1500 \mathrm{~m}$

Habitat:

Short, sclerophyllous, subelfin forest; associates include Melastoma, Actinodaphne, Meliosma,

Calamus, Daphniphyllum; soils serpentine.

\section{Field Char:}

Subdominant shrub, $2 \mathrm{~m}$ tall and wide, branching profusely, leaves dark green above, very pale green below; corolla white, androecium tan.

McDonald \& Ismail 4161

5 August 1993

HERBARIUM BOGORIENSE HARVARD UNIVERSITY HERBARIA 


\section{PLANTS OF INDONESIA}

Poaceae

Cat. \# 4162

\section{Poaceae}

Det. By: McDonald 1993

Locality:

Kabaena; Kabaena,

Summit of Sangia Wita, ca. $10 \mathrm{~km} \mathrm{SE}$ of Tangkeno.

$5^{\circ} 19^{\prime} \mathrm{S} 121^{\circ} 58^{\prime} \mathrm{E}$ Alt: $1500 \mathrm{~m}$

Habitat:

Short, sclerophyllous, subelfin forest; associates include Melastoma, Actinodaphne, Meliosma, Calamus, Daphniphyllum; soils serpentine.

Field Char:

Subdominant grass in open areas, clumped, 1-1.5 m tall, foliage green, inflorescence purplish during anthesis.

McDonald \& Ismail 4162

5 August 1993

\section{HERBARIUM BOGORIENSE \\ HARVARD UNIVERSITY HERBARIA}

\section{PLANTS OF INDONESIA}

Sapotaceae

Cat. \# 4163

\section{Sapotaceae}

Det. By: McDonald 1993

Locality:

Kabaena; Kabaena,

Gunung Sabampolulu, $1 \mathrm{~km}$ SSE of Tangkeno.

$5^{\circ} 16^{\prime} \mathrm{S} 121^{\circ} 56^{\prime} \mathrm{E}$ Alt: $700-900 \mathrm{~m}$

Habitat:

W slope of range, forest-savannah; arborescent associates include Grevillea, Helicia, Gardenia, Vaccinium, Eugenia. Soils serpentine.

\section{Field Char:}

Tree $14 \mathrm{~m}$ tall, branching above, trunk cylindrical, $30 \mathrm{~cm}$ diam; bark grayish brown, somewhat smooth, latex white; fruits depressed rotund, light green, somewhat glossy.

Notes:

NCI voucher

McDonald \& Ismail $4163 \quad 6$ August 1993

HERBARIUM BOGORIENSE HARVARD UNIVERSITY HERBARIA 


\section{PLANTS OF INDONESIA}

Annonaceae

Cat. \# 4164

Desmos chinensis Lour.

Det. By: McDonald 1993

Locality:

Kabaena; Kabaena,

Gunung Sabampolulu, $1 \mathrm{~km}$ SSE of Tangkeno.

$5^{\circ} 16^{\prime} \mathrm{S} 121^{\circ} 56^{\prime} \mathrm{E}$ Alt: $700-900 \mathrm{~m}$

Habitat:

W slope of range, forest-savannah; arborescent associates include Grevillea, Helicia, Gardenia, Vaccinium, Eugenia. Soils serpentine.

Field Char:

Liana $7 \mathrm{~m}$ tall, much branched; stems twining, to 10

m long; basal stem ca. $4 \mathrm{~cm}$ diam; bark dark gray;

tepals in 2 series, $3-5 \mathrm{~cm}$ long, $1-1.5 \mathrm{~cm}$ wide,

coriaceous, yellow-green.

Notes:

$\mathrm{NCI}$ voucher

McDonald \& Ismail 4164

6 August 1993

HERBARIUM BOGORIENSE

HARVARD UNIVERSITY HERIARIA

\section{PLANTS OF INDONESIA}

Cat. \# 4165

Cratoxylon formosum (Jack) Dyer

Det. By: McDonald 1993

Locality:

Kabaena; Kabaena,

Gunung Sabampolulu, $1 \mathrm{~km}$ SSE of Tangkeno.

$5^{\circ} 16^{\prime} \mathrm{S} 121^{\circ} 56^{\prime} \mathrm{E}$ Alt: $700-900 \mathrm{~m}$

Habitat:

W slope of range, forest-savannah; arborescent associates include Grevillea, Helicia, Gardenia, Vaccinium, Eugenia. Soils serpentine.

Field Char:

Short tree $5 \mathrm{~m}$ tall, branching above and below;

leaves young, green and red; sepals maroon outside, corolla pale pink, androecium yellowish.

McDonald \& Ismail 4165

6 August 1993

HERBARIUM BOGORIENSE HARVARD UNIVERSITY HERBARIA 


\section{PLANTS OF INDONESIA}

Nepenthaceae

Nepenthes $c f$. maxima Nees

N. maxima Nees det. K.K. McDonmell

Det. By: McDonald 1993 ture 1998

Locality:

Kabaena; Kabaena,

Gunung Sabampolulu, $1 \mathrm{~km}$ SSE of Tangkeno.

$5^{\circ} 16^{\prime} \mathrm{S} 121^{\circ} 56^{\prime} \mathrm{E}$ Alt: $700-900 \mathrm{~m}$

\section{Habitat:}

W slope of range, forest-savannah; arborescent associates include Grevillea, Helicia, Gardenia, Vaccinium, Eugenia. Soils serpentine.

Field Char:

Solitary, terrestrial herb, rarely branching, $30 \mathrm{~cm}$ tall, forming distended rosettes; lamina-petiole green or bronze, pitcher green and maroon, mottled, with two parallel, rows of maroon cilia, lip maroonstriate.

McDonald \& Ismail 4166

6 August 1993

HERBARIUM BOGORIENSE

HARVARD UNIVERSITY HERBARIA

\section{PLANTS OF INDONESIA}

Euphorbiaceae

Cat. \# 4167

\section{Glochidion sp.}

Det. By: McDonald 1993

Locality:

Kabaena; Kabaena,

Gunung Sabampolulu, $1 \mathrm{~km}$ SSE of Tangkeno.

$5^{\circ} 16^{\prime} \mathrm{S} 121^{\circ} 56^{\prime} \mathrm{E}$ Alt: $700-900 \mathrm{~m}$

Habitat:

W slope of range, forest-savannah; arborescent associates include Grevillea, Helicia, Gardenia, Vaccinium, Eugenia. Soils serpentine.

Field Char:

Short tree $5 \mathrm{~m}$ tall, branching above; trunk cylindrical, bark pale gray; corolla yellow, fruits pink.

McDonald \& Ismail 4167

HERBARIUM BOGORIENSE HARVARD UNIVERSITY HERBARIA 


\section{PLANTS OF INDONESIA}

Orchidaceae

Cat. \# 4168

\section{Orchidaceae}

Det. By: McDonald 1993

Locality:

Kabaena; Kabaena,

Gunung Sabampolulu, $1 \mathrm{~km}$ SSE of Tangkeno.

$5^{\circ} 16^{\prime} \mathrm{S} 121^{\circ} 56^{\prime} \mathrm{E}$ Alt: $700-900 \mathrm{~m}$

Habitat:

W slope of range, forest-savannah; arborescent associates include Grevillea, Helicia, Gardenia, Vaccinium, Eugenia. Soils serpentine.

Field Char:

Terrestrial orchid, common in grass; foliage light green, scape reaching 1-1.5 m tall, peduncle purple, ovary purple, tepals purplish, keel yellowish inside.

McDonald \& Ismail 4168

6 August 1993

\section{HERBARIUM BOGORIENSE \\ HARVARD UNIVERSITY HERBARIA}

\section{PLANTS OF INDONESIA}

Rubiaceae

Cat. \# 4169

Gardenia sp.

Det. By: McDonald 1993

Locality:

Kabaena; Kabaena,

Gunung Sabampolulu, $1 \mathrm{~km}$ SSE of Tangkeno.

$5^{\circ} 16^{\prime} \mathrm{S} 121^{\circ} 56^{\prime} \mathrm{E}$ Alt: $700-900 \mathrm{~m}$

Habitat:

W slope of range, forest-savannah; arborescent associates include Grevillea, Helicia, Gardenia, Vaccinium, Eugenia. Soils serpentine.

Field Char:

Common shrubby tree on forest margin; branching above, $5 \mathrm{~m}$ tall; calyx pale green, corolla white, turning yoke after anthesis; fruits orange inside, grayish green outside, seeds red.

McDonald \& Ismail 4169

6 August 1993

HERBARIUM BOGORIENSE HARVARD UNIVERSITY HERBARIA 


\section{PLANTS OF INDONESIA}

Nepenthaceae

Cat. \# 4170

Nepenthes mirabiliss (Lour.) Merr.

maxima Nees.

maxima Nees.
Det. By: MeDertald1993 K.K. McDonnall 1998

Locality:

Kabaena; Kabaena,

Gunung Sabampolulu, $1 \mathrm{~km}$ SSE of Tangkeno.

$5^{\circ} 16^{\prime} \mathrm{S} 121^{\circ} 56^{\prime} \mathrm{E}$ Alt: $700-900 \mathrm{~m}$

Habitat:

W slope of range, forest-savannah; arborescent associates include Grevillea, Helicia, Gardenia, Vaccinium, Eugenia. Soils serpentine.

Field Char:

Liana in open, shrubby areas, stems to $7 \mathrm{~m}$ long, lamina light green, pitchers pale yellow-green, the throat sparingly purple striate or mottled, lip purplemottled inside, light yellow green above.

McDonald \& Ismail 4170

6 August 1993

HERBARIUMM BOGORIENSE

HARVARD UNIVERSITY HERBARIA

\section{PLANTS OF INDONESIA}

Rubiaceae

Cat. \# 4171

Timonius sp.

Det. By: McDonald 1993

Locality:

Kabaena; Kabaena,

Gunung Sabampolulu, $1 \mathrm{~km}$ SSE of Tangkeno.

$5^{\circ} 16^{\prime} \mathrm{S} 121^{\circ} 56^{\prime} \mathrm{E}$ Alt: $700-900 \mathrm{~m}$

Habitat:

W slope of range, forest-savannah; arborescent associates include Grevillea, Helicia, Gardenia, Vaccinium, Eugenia. Soils serpentine.

Field Char:

Short tree $5 \mathrm{~m}$ tall, branching above; ovary green,

corolla white, hypocrateriform, segments 6 .

McDonald \& Ismail 4171

6 August 1993

HERBARIUM BOGORIENSE

HARVARD UNIVERSITY HERBARIA 


\section{PLANTS OF INDONESIA}

Dilleniaceae

Cat. \# 4172

Tetracera scandens (L). Merr.

Det. By: McDonald 1993

Locality:

Kabaena; Kabaena,

Gunung Sabampolulu, $1 \mathrm{~km}$ SSE of Tangkeno.

$5^{\circ} 16^{\prime} \mathrm{S} 121^{\circ} 56^{\prime} \mathrm{E}$ Alt: $700-900 \mathrm{~m}$

Habitat:

W slope of range, forest-savannah; arborescent associates include Grevillea, Helicia, Gardenia, Vaccinium, Eugenia. Soils serpentine.

Field Char:

Common shrub or liana, stems $4 \mathrm{~m}$ long, calyx light green, fruits reddish.

McDonald \& Ismail 4172

6 August 1993

\section{HERBARIUM BOGORIENSE HARVARD UNIVERSITY HERBARIA}

\section{PLANTS OF INDONESIA}

Cat. \# 4173

Fern

Det. By: McDonald 1993

Locality:

Kabaena; Kabaena,

Gunung Sabampolulu, $1 \mathrm{~km}$ SSE of Tangkeno.

$5^{\circ} 16^{\prime} \mathrm{S} 121^{\circ} 56^{\prime} \mathrm{E}$ Alt: $700-900 \mathrm{~m}$

Habitat:

W slope of range, forest-savannah; arborescent associates include Grevillea, Helicia, Gardenia, Vaccinium, Eugenia. Soils serpentine.

Field Char:

Terrestrial fern on exposed areas devoid of all plants but grasses, sori marginal.

McDonald \& Ismail 4173

6 August 1993

HERBARIUM BOGORIENSE HARVARD UNIVERSITY HERBARIA 


\section{PLANTS OF INDONESIA}

Flacourtiaceae

Cat. \# 4174

Scolopia spinosa (Roxb.) Warb.

Det. By: McDonald 1993

Locality:

Kabaena; Kabaena,

Gunung Sabampolulu, $1 \mathrm{~km}$ SSE of Tangkeno.

$5^{\circ} 16^{\prime} \mathrm{S} 121^{\circ} 56^{\prime} \mathrm{E}$ Alt: $700-900 \mathrm{~m}$

Habitat:

W slope of range, forest-savannah; arborescent associates include Grevillea, Helicia, Gardenia, Vaccinium, Eugenia. Soils serpentine.

Field Char:

Small tree $5 \mathrm{~m}$ tall, branching above and below, leaves green above, light green below, calyx pale yellow-green, stamens white.

McDonald \& Ismail 4174

6 August 1993

\section{HERBARIUM BOGORIENSE HARVARD UNIVERSITY HERBARIA}

\section{PLANTS OF INDONESIA}

Orchidaceae

Cat. \# 4175

\section{Orchidaceae}

Det. By: McDonald 1993

Locality:

Kabaena; Kabaena,

Gunung Sabampolulu, $1 \mathrm{~km}$ SSE of Tangkeno.

$5^{\circ} 16^{\prime} \mathrm{S} 121^{\circ} 56^{\prime} \mathrm{E}$ Alt: $700-900 \mathrm{~m}$

Habitat:

W slope of range, forest-savannah; arborescent associates include Grevillea, Helicia, Gardenia, Vaccinium, Eugenia. Soils serpentine.

Field Char:

Terrestrial orchid growing in grass, stems erect, to $40 \mathrm{~cm}$ tall; lamina green or purplish; tepals pale lavender and white, forming a basal spur, column and corolla tube purple-dotted.

McDonald \& Ismail 4175

6 August 1993

HERBARIUM BOGORIENSE HARVARD UNIVERSITY HERBARIA 


\section{PLANTS OF INDONESIA}

Cyperaceae

Cat. \# 4176

Scleria scrobiculata Nees \& May.

Det. By: McDonald 1993

Locality:

Kabaena; Kabaena,

Gunung Sabampolulu, $1 \mathrm{~km}$ SSE of Tangkeno.

$5^{\circ} 16^{\prime} \mathrm{S} 121^{\circ} 56^{\prime} \mathrm{E}$ Alt: $700-900 \mathrm{~m}$

Habitat:

W slope of range, forest-savannah; arborescent associates include Grevillea, Helicia, Gardenia, Vaccinium, Eugenia. Soils serpentine.

Field Char:

Clumping herb ca. $1 \mathrm{~m}$ tall, basal stem brown, leaves light green, inflorescence dark brown; fruit glossy white.

McDonald \& Ismail 4176

6 August 1993

HERBARIUM BOGORIENSE
HARVARD UNIVERSITY HERBARIA

\section{PLANTS OF INDONESIA}

Asteraceae

Cat. \# 4177

Asteraceae

Det. By: McDonald 1993

Locality:

Kabaena; Kabaena,

Gunung Sabampolulu, $1 \mathrm{~km}$ SSE of Tangkeno.

$5^{\circ} 16^{\prime} \mathrm{S} 121^{\circ} 56^{\prime} \mathrm{E}$ Alt: $700-900 \mathrm{~m}$

Habitat:

W slope of range, forest-savannah; arborescent associates include Grevillea, Helicia, Gardenia, Vaccinium, Eugenia. Soils serpentine.

Field Char:

Occasional weedy herb, to $20 \mathrm{~cm}$ tall; phyllaries green, corollas yellowish, remaining enveloped by involucre.

McDonald \& Ismail 4177

6 August 1993

HERBARIUM BOGORIENSE HARVARD UNIVERSITY HERBARIA 


\section{PLANTS OF INDONESIA}

Poaceae

Cat. \# 4178

Poaceae

Det. By: McDonald 1993

Locality:

Kabaena; Kabaena,

Gunung Sabampolulu, $1 \mathrm{~km}$ SSE of Tangkeno.

$5^{\circ} 16^{\prime} \mathrm{S} 121^{\circ} 56^{\prime} \mathrm{E}$ Alt: $700-900 \mathrm{~m}$

Habitat:

W slope of range, forest-savannah; arborescent associates include Grevillea, Helicia, Gardenia, Vaccinium, Eugenia. Soils serpentine.

Field Char:

Subdominant cespitose grass.

McDonald \& Ismail 4178

6 August 1993

HERBARIUM BOGORIENSE

HARVARD UNIVERSITY HERBARIA

\section{PLANTS OF INDONESIA}

Poaceae

Cat. \# 4179

Poaceae

Det. By: McDonald 1993

Locality:

Kabaena; Kabaena,

Gunung Sabampolulu, $1 \mathrm{~km}$ SSE of Tangkeno.

$5^{\circ} 16^{\prime}$ 'S $121^{\circ} 56^{\prime}$ E Alt: $700-900$ m

Habitat:

W slope of range, forest-savannah; arborescent associates include Grevillea, Helicia, Gardenia, Vaccinium, Eugenia. Soils serpentine.

Field Char:

Occasional, short annual grass.

McDonald \& Ismail 4179

6 August 1993

HERBARIUM BOGORIENSE HARVARD UNIVERSITY HERBARIA 


\section{PLANTS OF INDONESIA}

Poaceae

Cat. \# 4180

Poaceae

Det. By: McDonald 1993

Locality:

Kabaena; Kabaena,

Gunung Sabampolulu, $1 \mathrm{~km}$ SSE of Tangkeno.

$5^{\circ} 16^{\prime} \mathrm{S} 121^{\circ} 56^{\prime} \mathrm{E}$ Alt: $700-900 \mathrm{~m}$

Habitat:

W slope of range, forest-savannah; arborescent associates include Grevillea, Helicia, Gardenia, Vaccinium, Eugenia. Soils serpentine.

Field Char:

Dominant, stoloniferous grass.

McDonald \& Ismail 4180

6 August 1993

\section{HERBARIUM BOGORIENSE HARVARD UNIVERSITY HERBARIA}

\section{PLANTS OF INDONESIA}

Fabaceae

Cat. \# 4181

Fabaceae Derris sp.

Det. By: McDonald 1993 J. Barham (K) April 1998

Locality:

Kabaena; Kabaena,

Gunung (Mt.) Katopi, $18 \mathrm{~km} \mathrm{NW}$ of Tangkeno.

$5^{\circ} 12^{\prime} \mathrm{S} 121^{\circ} 25^{\prime} \mathrm{E}$ Alt: $250-500 \mathrm{~m}$

Habitat:

Open, short-tree savannah on serpentine soils, grasses dominant, shrubs include Grevillea, Metrosideros, Pandanus, and Cycas.

Field Char:

Common, woody shrub 1-2 m tall, 2-3 m diam, branching above and below, foliage light green, calyx pale green and reddish, corollas white.

McDonald \& Ismail 4181

7 August 1993

HERBARIUM BOGORIENSE HARVARD UNIVERSITY HERBARIA 


\section{PLANTS OF INDONESIA}

Sterculiaceae

Cat. \# 4182

Helicteres angustifolia $L$.

Det. By: McDonald 1993

Locality:

Kabaena; Kabaena,

Gunung (Mt.) Katopi, $18 \mathrm{~km} \mathrm{NW}$ of Tangkeno.

$5^{\circ} 12^{\prime} \mathrm{S} 121^{\circ} 25^{\prime} \mathrm{E}$ Alt: $250-500 \mathrm{~m}$

Habitat:

Open, short-tree savannah on serpentine soils, grasses dominant, shrubs include Grevillea, Metrosideros, Pandanus, and Cycas.

Field Char:

Common short shrub, $1 \mathrm{~m}$ tall, branching sparingly

from base, foliage only on branch tips, leaves greenish above, tan-pubescent below, calyx canescent, corolla pale blue; fruit glandulartomentose, dehiscent, carpels 5 .

McDonald \& Ismail 4182

7 August 1993

\section{HERBARIUM BOGORIENSE \\ HARVARD UNIVERSITY HERBARIA}

\section{PLANTS OF INDONESIA}

Pandanaceae

Cat. \# 4183

\section{Pandanus}

Det. By: McDonald 1993

Locality:

Kabaena; Kabaena,

Gunung (Mt.) Katopi, $18 \mathrm{~km} \mathrm{NW}$ of Tangkeno.

$5^{\circ} 12^{\prime} \mathrm{S} 121^{\circ} 25^{\prime} \mathrm{E}$ Alt: $250-500 \mathrm{~m}$

Habitat:

Open, short-tree savannah on serpentine soils, grasses dominant, shrubs include Grevillea, Metrosideros, Pandanus, and Cycas.

Field Char:

Common short tree, $5 \mathrm{~m}$ tall, branching sparingly above and below, stems $>4 \mathrm{~cm}$ diam; inflorescence

$20 \mathrm{~cm}$ long, secondary staminate branches

cylindrical, $5 \mathrm{~cm}$ long, $2 \mathrm{~cm}$ diam, branches

elliptical of equal size; fruit immature, subrotund, $\mathrm{ca}$.

$7 \mathrm{~cm}$ diam.

McDonald \& Ismail 4183

7 August 1993

HERBARIUM BOGORIENSE HARVARD UNIVERSITY HERBARIA 


\section{PLANTS OF INDONESIA}

Cycadaceae

Cat. \# 4184

Cycas rumphii Miq.

Det. By: McDonald 1993

Locality:

Kabaena; Kabaena,

Gunung (Mt.) Katopi, $18 \mathrm{~km} \mathrm{NW}$ of Tangkeno.

$5^{\circ} 12^{\prime} \mathrm{S} 121^{\circ} 25^{\prime} \mathrm{E}$ Alt: $250-500 \mathrm{~m}$

Habitat:

Open, short-tree savannah on serpentine soils, grasses dominant, shrubs include Grevillea, Metrosideros, Pandanus, and Cycas.

Field Char:

Short tree, occasionally branching 2-3 times at base, trunk to $30 \mathrm{~cm}$ diam, to $2.5 \mathrm{~m}$ tall, foliage apical, in rosette, primordial leaves tan-tomentose; fronds 2-3

$\mathrm{m}$ long; fertile leaves rusty-tomentose, leaves olive.

McDonald \& Ismail 4184

7 August 1993

HERBARIUM BOGORIENSE

HARVARD UNIVERSITY HERBARIA

\section{PLANTS OF INDONESIA}

Celastraceae

Cat. \# 4185

Salacia korthalsiana Miq.

Det. By: McDonald 1993

Locality:

Kabaena; Kabaena,

Gunung (Mt.) Katopi, $18 \mathrm{~km} \mathrm{NW}$ of Tangkeno.

$5^{\circ} 12^{\prime} \mathrm{S} 121^{\circ} 25^{\prime} \mathrm{E}$ Alt: $250-500 \mathrm{~m}$

Habitat:

Open, short-tree savannah on serpentine soils, grasses dominant, shrubs include Grevillea, Metrosideros, Pandanus, and Cycas.

Field Char:

Common shrub $1.5 \mathrm{~m}$ tall, $2 \mathrm{~m}$ wide, branching much; fruits green.

Notes:

$\mathrm{y}$

McDonald \& Ismail 4185

7 August 1993

HERBARIUM BOGORIENSE HARVARD UNIVERSITY HERBARIA 


\section{PLANTS OF INDONESIA}

Euphorbiaceae

Cat. \# 4186

Glochidion sp.

Det. By: McDonald 1993

Locality:

Kabaena; Kabaena,

Gunung (Mt.) Katopi, $18 \mathrm{~km}$ NW of Tangkeno.

$5^{\circ} 12^{\prime} \mathrm{S} 121^{\circ} 25^{\prime} \mathrm{E}$ Alt: $250-500 \mathrm{~m}$

Habitat:

Open, short-tree savannah on serpentine soils, grasses dominant, shrubs include Grevillea, Metrosideros, Pandanus, and Cycas.

Field Char:

Shrub $2 \mathrm{~m}$ tall, branching above, fruits green and immature.

McDonald \& Ismail 4186

7 August 1993

HERBARIUM BOGORIENSE HARVARD UNIVERSITY HERBARIA

\section{PLANTS OF INDONESIA}

Poaceae

Cat. \# 4187

Poaceae

Det. By: McDonald 1993

Locality:

Kabaena; Kabaena,

Gunung (Mt.) Katopi, $18 \mathrm{~km} \mathrm{NW}$ of Tangkeno.

$5^{\circ} 12^{\prime} \mathrm{S} 121^{\circ} 25^{\prime} \mathrm{E}$ Alt: $250-500 \mathrm{~m}$

Habitat:

Open, short-tree savannah on serpentine soils, grasses dominant, shrubs include Grevillea, Metrosideros, Pandanus, and Cycas.

Field Char:

Dominant grass, $1 \mathrm{~m}$ tall, stems to $2 \mathrm{~m}$ long, branching above and at base; inflorescence covered with white (fungal?) growth.

McDonald \& Ismail 4187

7 August 1993

HERBARIUM BOGORIENSE HARVARD UNIVERSITY HERBARIA 


\section{PLANTS OF INDONESIA}

Cat. \# 4188

\section{Barliera grionitis}

Det. By:

Locality:

Kabaena; Kabaena,

Mountain ridge between Tangkeno and Batu Sangia, 1-3 km W of Tangkeno.

$5^{\circ} 16^{\prime} \mathrm{S} 121^{\circ} 56^{\prime} \mathrm{E}$ Alt: $700-800 \mathrm{~m}$

Habitat:

Secondary forest around ladangs. Associates include Arenga, bamboos, Ficus, Trichospermum, Albizia, etc.

Field Char:

Uncommon understory shrub, $1 \mathrm{~m}$ tall, branching sparingly above and below, new growth of stems green, spines white; corolla orange, stamens 2 , exserted.

McDonald \& Ismail 4188

7 August 1993

HERBARIUM BOGORIENSE HARVARD UNIVERSITY HERBARIA 